\title{
Abstracts from the Second European Workshop on Exo/Astrobiology
}

\author{
16-19 September 2002 \\ Graz, Austria
}

\begin{abstract}
Scientific objectives
One of the main objectives of many of the international planetary exploration programmes of the twenty-first century is the search for traces of present or fossil life in the solar system and beyond. Three possible main candidates for the search for microbial life forms on a planetary body in the solar system were identified at the First European Workshop on Exo/Astrobiology in May 2001 at ESA/ESRIN, Frascati, Italy. Possible habitats are the surface and near subsurface of Mars, the putative subsurface water ocean of Jupiter's moon Europa and - also more speculative - the interior of Saturn's largest satellite Titan. In addition, Titan's atmosphere and surface provide a reference laboratory to study, by default, the role of liquid water in exobiology.

The Workshop in Graz will deal on the formation of organic molecules in planetary nebulae, the study of organics in comets, asteroids, meteoroids and interstellar dust particles, the evolution of planetary atmospheres in relation to the origin of life, as well as the history of the solar particle and radiation environment in our solar system and the implications for space exposure experiments and planetary evolution. Since, the Space Research Institute (IWF) of the Austrian Academy of Sciences, as the main local organizer, has a long tradition and many years of experience in planetary exploration and is involved in Exo/ Astrobiology relevant missions like Cassini/Huygens, Rosetta, MarsExpress, Nozomi, Netlander and space simulation experiments, the Workshop will focus on the exploration of Mars, Titan and Europa. Terrestrial permafrost is a very good habitat for micro-organisms on Earth, therefore, a special session is dedicated to Astrobiology relevant permafrost studies. On the perspectives opened by the discovery of putative terrestrial extra-solar planets the workshop will also discuss this new, exciting and growing field.
\end{abstract}

Since most European countries participate in ESA's AURORA and ELIPS programmes, sessions dedicated to planetary and space exploration technologies relevant to Exo/Astrobiology are also organized. The main objective of the Workshop is to promote a constructive and interdisciplinary scientific cooperation in the growing field of Exo/ Astrobiology. More specifically, the Workshop aims to:

- Present the latest results in the fast-developing fields of studying the origins of life, planetary exploration and the search for extra-solar planets related to Exo/Astrobiology.

- Favour the cooperation between scientists and research groups of different scientific disciplines, like planetology, astronomy, astrophysics, geology, atmospheric physics, chemistry, biophysics, biochemistry, microbiology, genetics, medicine, robotics, etc.

- Bring together scientists, spacecraft engineers and industrial partners for planning future space missions relevant to planetary exploration.

- Highlight the scientific, technical, industrial and philosophical benefits for the public.

The Workshop is organized as a forum to maximize the advantages of interdisciplinary innovative discussions. Poster sessions will be open during the whole meeting and Proceedings will by published as an ESA Special Publication (ESA-SP-518) after the Workshop.

Helmut Lammer (main local organizer)

Austrian Academy of Sciences, Space Research Institute (IWF), Department of Extraterrestrial Physics, Planetary Atmosphere Group, Graz, Austria.

\section{EANA first Anniversary by André Brack, President}

EANA, the European Exo/Astrobiology Network Association, was created in 2001 to co-ordinate the different European centres of excellence in Exo/Astrobiology or related fields already organized in national networks. Exo/Astrobiology encompasses the disciplines of chemistry, biology, palaeontology, geology, atmospheric physics, planetary physics and stellar physics. Most aspects of this research require substantial resources in terms of analytical or simulation laboratory facilities, ground instrumentation, spacecraft and staffing. There are several centres of excellence in Exo/Astrobiology or related fields of research in Europe and several nations have established national networks, which were not seriously co-ordinated for the sharing of expertise and facilities on a European level.

The specific objectives of EANA are to bring together European researchers interested in Exo/Astrobiology programmes and to foster their co-operation, to attract young scientists to this quickly evolving interdisciplinary field of research, to create a website establishing a database of expertise in different aspects of Exo/Astrobiology, to interface the Network with European bodies such as ESA, ESF, the European Commission and with non-European institutions active in the field, and to popularize Exo/Astrobiology to the public and to students. It is run by an Executive Council consisting of national members presently representing twelve European nations active in the field (Austria, Belgium, Denmark, France, Germany, Italy, Portugal, Spain, Sweden, Switzerland, The Netherlands, United Kingdom).

Since its creation, EANA co-organized with ESA the First European Exo/Astrobiology meeting in Frascati, Italy. During the Frascati Workshop, from 21 to 23 May 2001, attended by 200 scientists, the national and international activities in Exo/Astrobiology were presented as well as the European achievements in the different fields covered by Exo/Astrobiology: the ingredients of life and chemistry of primitive life, life in the extremes and terrestrial analogues for extraterrestrial habitats, extraterrestrial/extra-solar habitability, the nature of, and the search for life in the solar system and beyond, and missions to search for life in the solar system. Both plenary, splinter and poster sessions generated lively discussions, which cemented the European community. The scientific input has been published by ESA as an ESA Special Publication (SP-496, 2001).

The EANA Web Page is under construction. It will be hosted as part of the ESA pilot Virtual Institute at ESTEC in Noordwijk, The Netherlands. 
To strengthen its networking action, EANA has submitted a proposal entitled 'The Limits of Planetary life: Origins and distribution' in the framework of the European Science Foundation Scientific Network Scheme. EANA has also submitted an Expression of Interest for a Network of Excellence of the European Commission 6th Framework Program in Astrobiology entitled 'European Astrobiology Space Science and Technology Network (EASSTN)'. With respect to the European Commission COST Actions to foster co-operation in a specific research area, COST D27 'Origin of life and early evolution' has been approved in Brussels for a period of five years. The main objective of this action is to develop the chemistry of the origins and the early evolution of life.

In the future, EANA anticipates including other European countries active in Exo/Astrobiology. Moreover, the creation of an international Astrobiology group to facilitate international exchange between established bodies dedicated to Astrobiology (i.e. organizations, societies and structures including EANA) and to harmonize the planning of joint Astrobiology meetings is in progress.

For the present European Exo/Astrobiology Workshop held in Graz, Austria special attention has been given to the planetology aspects of Exo/Astrobiology to acknowledge the expertise of the local organizers. Workshop organization

The Second European Workshop on Exo/Astrobiology is co-organized by the Planetary Atmosphere Group of the Department of Extraterrestrial Physics, of the Space Research Institute (IWF) of the Austrian Academy of Sciences, the Astrophysics Group of the Institute of Geophysics, Astrophysics and Meteorology (IGAM) of the University of Graz, the European Exo/Astrobiology Network Association (EANA) and the European Space Agency (ESA).

Organizing committee

H. Lammer, (Scientific coordination), IWF, Austrian Academy of Sciences, Austria

I. Jetzl, C. Kolb, A. Scherr, W. Voller, S. Zehetleitner, IWF, Austrian

Academy of Sciences, Austria
R. Leitinger, A. Hanslmeier, IGAM, University of Graz, Austria

A. Brack, CBM-CNRS Orléans, France

L. Colangeli, Osservatorio di Capodimonte, Italy

P. Ehrenfreund, Observatory Leiden, The Netherlands

B. Hofmann, Natural History Museum, Bern, Switzerland

A. Penny, Rutherford Appleton Laboratory, UK

J. Perez Mercader, Centro de Astrobiologia, Madrid, Spain

P. Clancy, ESA

Science advisory committee

R. Amils, Centro de Astrobiologia, Madrid, Spain

O. Angerer, ESA

M. Coradini, ESA

C. Cockell, British Antarctic Survey, UK

C. Cosmovici, IFSI CNR, Italy

L. Cruzeiro-Hansson, University de Algarve, Portugal

D. Field, University of Aarhus, Denmark

M. Fridlund, ESA

A. Hjalmarson, Onsala Space Observatory, Sweden

N. Holm, University of Stockholm, Sweden

G. Horneck, DLR, Cologne, Germany

G. von Kiedrowski, University of Bochum, Germany

J. M. Knudsen, Orsted Laboratory, Denmark

R. Paepe, Geobound International, Brussels, Belgium

D. Prieur, Université de Bretagne Occidentale, France

F. Raulin, CNRS, LISA Universite Paris, France

G. Ronto, MTA-SE, Semmelweis University, Budapest, Hungary

H. O. Rucker, IWF, Austrian Academy of Sciences, Austria

G. Schwehm, ESA

H. Stan-Lotter, University of Salzburg, Austria

K. Stetter, University of Regensburg, Germany

J.-C. Worms, European Science Foundation (ESF)

\section{Exo/Astrobiology Activities by NASA and ESA}

\section{Exobiology, the ELIPS and AURORA programmes P. Clancy, D. Schmitt, O. Angerer Directorate of Manned Spaceflight and Microgravity, ESA, European Space Agency, ESA/ESTEC, Noordwijk, The Netherlands, E-mail: paul.clancy@esa.int}

The ESA activities in the area of exobiology that have been ongoing for a number of years received a boost in November 2001 when the European Ministers responsible for space activities approved new funding for two programmes which will support future European exobiology activities. The European Life and Physical Sciences and Applications in Space (ELIPS) programme, covering research in those areas in the years 2002 to 2006, was subscribed at a level of $€ 171.4 \mathrm{~m}$. Although most of this will be dedicated to research on the International Space Station some will be available for support to exobiology beyond Low Earth Orbit (LEO). The Aurora programme that covers the formulation of a European long-term plan for the robotic and human exploration of the solar system bodies, in particular those holding promise for traces of life, was subscribed at a level of $€ 14.1 \mathrm{~m}$ for a preparatory phase for the period 2002 to 2004. Although these subscription rates were lower than ESA had proposed, (€320m in the case of ELIPS and $€ 40 \mathrm{~m}$ in the case of Aurora), they represent a commitment by European countries to continue supporting exobiological research and develop Europe into a significant partner in planetary exploration. ESA's activities in exobiology up to now have included experiments on the exposure of cellular systems, micro-organisms and yeasts to UV and space radiation (Eureca mission in 1992); radiation biology and dosimetry, extremophile space exposure and chemical evolution (FOTON flights in 1994, 1997, 1999 and 2002). Future LEO activities focus on the EXPOSE facility for Space Station covering exposure of organic samples (e.g. amino acids, PAH's, microalgal communities and spores). ESA-supported search for life activities have concentrated on definition studies on sample acquisition and analysis on the Martian surface. The ELIPS programme will support continuation of these activities including ground-based experiments. The proposed ExoMars mission for 2007 is presently being studied for execution of this search for life strategy. In a longer-term perspective the Aurora programme will prepare for future human Mars missions, by developing necessary technologies, conducting robotic missions to validate the technological developments and preparing scientific aspects connected to humans on long-duration missions, planetary protection and exobiology. The advisory body of Aurora, aided by external experts, presently defines the long-term plan, the principles of which will be fixed by the end of 2002. This will allow the selection of the first missions to be developed through Aurora. Independent of mission concepts selected, all missions will be used to support exobiological research, as this was identified as the topic that holds the largest interest for the European scientific community concerned with planetary exploration.

\section{The NASA Astrobiology Institute: reaching globally ... and beyond \\ R. A. Grymes \\ Directorate Astrobiology Institute NAI, NASA, MS 240-1 NASA Ames Research Center, Moffett Field, CA 94035, USA, E-mail: rgrymes@arc.nasa.gov}

The NASA Astrobiology Institute (NAI), is NASA's centerpiece investment in astrobiology. Now in its fifth year, the NAI's fifteen Lead Teams represent over 700 scientists engaged in interdisciplinary research addressing all the themed topics of this meeting. In addition to 
its emphasis on excellence and innovation in research, the Institute also focusses on influencing NASA's mission planning and technology investment strategies. Additionally, the NAI maintains a strong and continuing commitment to exploring remote collaboration tools, communicating with the public, and training the next generation of astrobiologists. The breadth of the Institute's science accomplishments, including highlights of recent discoveries, publications, field investigations, and planetary exploration involvement, is the central focus to be communicated to our colleagues of the international scientific community. The Institute has undertaken the development of an active program for international partners. As part of this effort, we recently initiated a mutual affiliation with the European Exo/Astrobiology
Network Association (EANA). It is particularly gratifying to watch as multiple bilateral partnerships grow into a circle of support between national organizations. NAI members are also strongly proactive on the editorial boards of all the journals supporting the field of astrobiology, are engaged in a variety of national and international professional societies, develop and distribute educational materials at all levels, and welcome the full participation of their colleagues in the supported Focus Groups of the NAI. Focus Groups concerned with planetary exploration strategic planning (Astromaterials, Mars, Europa, Titan) and interdisciplinary innovation (Mission to Early Earth, EcoGenomics, EvoGenomics) are currently active, with others proposed for the future.

\section{From Organic Molecules in Space via Planetary Evolution to the Earliest Organisms on Earth}

\section{Origin of planetary atmospheres and their role in the evolution of life \\ S. J. Bauer \\ Institute of Geophysics, Astrophysics and Meteorology, University of Graz, Universitätsplatz, 5A-8010 Graz, Austria, E-mail: \\ siegfried.bauer@uni-graz.at}

The successful demonstration by the Miller-Urey experiment, some fifty years ago, that a reducing gas mixture with an external supply of energy would lead to the synthesis of amino acids, was responsible for the 'standard model' of the Earth's early atmosphere that found broad acceptance for several decades. According to current understanding of atmospheric escape processes, such a reducing atmosphere, however, would have enjoyed only a relatively short lifetime. Already in 1951 geological evidence for mantle degassing of volatiles $\mathrm{H}_{2} \mathrm{O}, \mathrm{CO}_{2}$ and $\mathrm{N}_{2}$ was available that could later explain the predominance of $\mathrm{N}_{2}$ (with $\mathrm{O}_{2}$ coming later as the product of life), after large amounts of $\mathrm{CO}_{2}$ were sequestered as carbonates because of the presence of liquid water, whose early presence led to the so-called Faint Sun Paradox. Recent observations of early Sun-like stars, however, imply a greater luminosity of the early Sun than has been supposed previously. With a Faint Sun, even a much denser atmosphere of Mars would not have facilitated liquid water on its surface. For which there is at least indirect evidence, however, the early $\mathrm{CO}_{2} / \mathrm{N}_{2}$ atmosphere of Mars - most of it lost by now seems to have been exposed to liquid water only to a limited extent (in amount and/or time), otherwise the present atmosphere would have to be more enriched in $\mathrm{N}_{2}$, because of the formation of carbonates (as on Earth): in fact it has a similar $\mathrm{CO}_{2} / \mathrm{N}_{2}$ ratio as Venus, where because of high temperature and the absence of liquid water, all $\mathrm{CO}_{2}$ has remained in the atmosphere. Although such 'neutral' (non-reducing) atmospheres may not have been conductive to the synthesis of prebiotic molecules, they may nevertheless, together with liquid water, have provided a suitable environment for the evolution of life via the supply of such molecules from other sources, including extraterrestrial ones. While the Miller-Urey synthesis may not have occurred on early Earth, it could have been operating in other environments, such as on comets, meteorites, and Titan.

\section{Nucleobases in carbonaceous chondrites \\ O. Botta}

Institute of Chemistry, Astrobiology Laboratory Leiden,

Einsteinweg 55, P.O. Box 9502, 2300 RA Leiden, The Netherlands, E-mail: o.botta@chem.leidenuniv.nl

Purines and pyrimidines, including adenine, guanine, cytosine, thymine and uracil, play a major role in terrestrial biochemistry. They are central components of DNA and RNA, molecules that are used in the storage, transcription and translation of genetic information. The concentration of uracil ${ }^{1}$ and the purines adenine, guanine, xanthine and hypoxanthine ${ }^{2}$ were determined in the Murchison, Murray and Orgueil carbonaceous meteorites in total concentrations of up to 1.6 parts per million (ppm). We present first results from our laboratory investigation of nucleobases in carbonaceous chondrites using a High Performance Liquid Chromatography (HPLC) system equipped with a Diode-Array detector. These data are the first stage towards future investigation on the stable isotope ratios of these compounds that would help tracing their formation history.

References

${ }^{1}$ Stoks, P. G., Schwartz, A. W., Nature 282 (1979), 709-710.

${ }^{2}$ Stoks, P. G., Schwartz, A. W., Geochim. Cosmochim. Acta 45 (1981), 563-569.

\section{Astrophysical and astrochemical insights into the origin of life}

P. Ehrenfreund and the ISSI (International Space Science Institute, Bern) Team*: 'Prebiotic matter in space' Raymond and Beverly Sackler Laboratory for Astrophysics at Leiden Observatory, P.O. Box 9513, 2300 RA Leiden, The Netherlands, E-mail: pascale@strw.leidenuniv.nl

We review the physical and chemical processes that form and shape organic matter in space. In particular we discuss the chemical pathways of organic matter in the interstellar medium, their evolution in protoplanetary disks and their integration into solar system material. Furthermore we investigate the role of impacts and the delivery of organic matter to the prebiotic Earth. We report on recent astronomical searches for biogenic molecules and related laboratory experiments. These results provide evidence that the basic building blocks of life are widespread in planetary systems in our Milky Way and other galaxies. Finally we discuss the construction of the OMIS (Organic Matter In Space) database, which is currently compiled by the ISSI Team.

* ISSI 'Prebiotic Matter Team': P. Ehrenfreund, L. Becker, J. Blank, J. Brucato, L. Colangeli, S. Derenne, D. Despois, A. Dutrey, H. Fraaije, W. Irvine, A. Lazcano, T. Owen, F. Robert.

The young and restless Sun: the radiative and magnetic properties of the young Sun and influences on paleoplanetary environments

E. F. Guinan(1), I. Ribas $(1,2)$

(1) Department of Astronomy and Astrophysics, Villanova University, 800 Lancaster Ave. PA 19085 Villanova, USA, E-mail:edward.guinan@villanova.edu; (2)Departament d'Astronomia i Meteorologia, Universitat de Barcelona, Av. Diagonal 647, 08028 Barcelona, Spain

As part of the 'Sun in Time' program we discuss the radiative and magnetic properties of the young Sun. Reliable determinations of the young Sun's radiative and magnetic properties (luminosity, spectral irradiance, flare energies/frequencies, and solar winds etc.) are of crucial 
importance for the study of the evolution of planetary paleo-atmospheres and development of life in the solar system. Standard stellar evolutionary models constructed for the Sun, show that some $4.5 \mathrm{Gyr}$ ago, the young Sun was about $200 \mathrm{~K}$ cooler and $\sim 10 \%$ smaller than today and had an initial luminosity of $\mathrm{L} \sim 70 \%$ of the present Sun. So that in the early stages of the solar system, the young Sun's total irradiance was significantly diminished. The decreased luminosity of the younger Sun should have resulted in cooler Earth in the past. However, geological and fossil evidence indicate that the Earth's climate $~ 3-4$ billion years ago (when the Sun was fainter) was not significantly cooler than now and may have even been warmer. This problem has become known as the 'Faint Sun' paradox. However, the study of young solar analogues (G0-5 stars) indicates that the young Sun was rapidly rotating and correspondingly had a much more robust magnetic dynamo. The strong magnetic dynamo of the young Sun resulted in very strong coronal X-ray and EUV emissions up to several hundred times those of the present Sun and chromospheric and transition-region FUV-UV fluxes $\sim 5$ to $100 \times$ greater than present solar values. X-ray and EUV observations of the youngest solar analogues (with ages $<300 \mathrm{Myr}$ ) indicate that the young Sun had frequent XUV flares with energies up to a thousand times (E total $\sim 10^{34}-10^{35}$ ergs) more energetic than the most powerful solar flares observed today. Also, recent studies of related active stars indicate that the young Sun had strong winds with inferred plasma densities up to 1000 times the present Sun. Ground-based photometric (carried out with robotic telescopes) show that young solartype stars are heavily spotted (5-20\% surface coverage by spots) and have rotationally modulated light variations of $\sim 3-10 \%$. For comparison, the present Sun during the maximum of the sunspot cycle has dark spots typically covering $0.15-0.20 \%$ of its surface. Also long-term studies of the young Suns appear to have activity cycles similar in length to the Sun's eleven-year cycle but with much larger ranges in brightness than observed today for the Sun. We briefly discuss the effects of the young Sun's greatly enhanced dynamo generated XUV emissions, flares, and winds on the developing early solar system - in particular on the photochemical and photoionization evolution (and possible erosion) of early planetary atmospheres and ionospheres. We also discuss some possible resolutions of the Faint Sun paradox.

\section{Acknowledgements}

This research is supported by NASA FUSE Grants NAG 5-8985 \& NAG 5-10387 and EUVE Mini-Grant SA2085-26310, which we gratefully acknowledge.

\section{Interstellar versus atmospheric generation of prebiotic amino acids}

U.J. Meierhenrich(1), G. M. Munoz Caro(2), W. A. Schutte(2), B. Barbier(3), A. Arcones Segovia(2), H. Rosenbauer(4),

W. H.-P. Thiemann(1), A. Brack(3)

(1) Department of Physics and Chemistry, Bremen University,

Leobener Str., D-28359 Bremen, Germany, E-mail: mhenrich@ uni-bremen.de; (2) Raymond and Beverly Sackler Laboratory for Astrophysics at Leiden Observatory, P.O. Box 9513, 2300 Leiden, The Netherlands; (3) Centre de Biophysique Moléculaire, CNRS, Rue Charles Sadron, F-45160 Orléans, France; (4) Max-Planck-Institut für Aeronomie, Max-Planck-Str. 2, D-37189 Katlenburg-Lindau, Germany Until very recently, prebiotic amino acids were believed to be generated in the atmosphere of the early Earth, as successfully simulated by the Urey-Miller experiments. ${ }^{1}$ So far, chiral amino acids have never been detected in the interstellar medium. In order to mimic the physicochemical processes in interstellar ices for the preparation of the COSAC experiment onboard of the ROSETTA Lander, we deposited a representative gas mixture containing $\mathrm{H}_{2} \mathrm{O}, \mathrm{CO}_{2}, \mathrm{CO}, \mathrm{CH}_{3} \mathrm{OH}$, and $\mathrm{NH}_{3}$ on to an aluminium surface at $12 \mathrm{~K}$ under high vacuum, $10^{-7}$ mbar. During deposition the molecules were subjected to ultraviolet radiation mainly at Lyman-a. After warm-up, hydrolysis and derivatization of the sample, we were able to identify 16 amino acids as well as furans and pyrroles in the interstellar ice analogues. ${ }^{2}$ The results were confirmed by parallel experiments using ${ }^{13} \mathrm{C}$-labelled ice analogues in order to exclude contamination. The separated and quantified enantiomers of the amino acids showed racemic occurrence. An interstellar origin of amino acids is now discussed to be an interesting alternative to the Urey-Miller mechanism. The advantages of the interstellar model will be introduced. References

${ }^{1}$ Miller S. L., Production of amino acids under possible primitive earth conditions. Science 117 (1953), 528.

${ }^{2}$ Muñoz Caro G. M., Meierhenrich U. J., Schutte W. A., Barbier B., Arcones Segovia A., Rosenbauer H., Thiemann W. H.-P., Brack A., Greenberg J. M., Amino acids from ultraviolet irradiation of interstellar ice analogues. Nature 416 (2002), 403-406.

\section{Evidence for water ice bearing provinces on Mars: update from HEND measurements on board NASA Odyssey Orbiter \\ I. Mitrofanov}

Russian Academy of Sciences, Institute for Space Research IKI,

Moscow, Russia, E-mail: imitrofa@space.ru

An update of the data obtained by the Russian High Energy Neutron Detector (HEND) is presented. HEND is part of the Gamma-Ray Spectrometer facility of NASA's Mars Odyssey mission. The evidence of large water ice-bearing areas is discussed, which are visible by our instrument both at the southern and northern hemispheres of Mars.

\section{The hunt for evidence of early life: a Paleobiologist's view of exobiology \\ J. W. Schopf}

Department of Earth and Space Sciences, Institute of Geophysics and Planetary Physics (Center for the Study of the Evolution and Origin of Life) and Molecular Biology Institute, University of California, Los Angeles, CA 90095-1567, USA CSEOL Geology Building Univ. Calif., Los Angeles, CA 90095-1567, USA,E-mail: schopf@ess.ucla.edu

The emergent field of Exo/Astrobiology - an inquiry into the origin and evolution of almost absolutely everything - is difficult and demanding. But the challenges it presents are not due simply to the all-inclusive questions the field addresses. Rather, their root cause, I think, lies in our own deficiencies, in the inadequacies of those of us who are practitioners of the science. Consider, for a moment, the nature of Nature. The natural world is neither entirely physical nor wholly biological, yet in our institutions we divide this world into two great camps - the physical sciences and the life sciences - populated by tribes of such different backgrounds and divergent interests that they barely speak the same language. At most universities the two tribes are even partitioned into separate 'homelands' the borders of which, though claimed to be open to all, are actually seldom crossed. Each of us, all products of this system, bears allegiance to one or the other of the two great tribes. And while this tribalism has simplified our lives - learning the ropes in a single discipline being far easier than grappling with many - we each also have paid a price. For Exo/Astrobiologic science, the cost has been high. The real world is an interdependent mix of the physical and life sciences; not one; not the other; but both. Despite our predilections to the contrary, Nature is not compartmentalized! Unless we find ways to better bridge the chasm that separates the sciences, our field will suffer. Rather than dwell on mistakes that could have been avoided had the chasm between the sciences been better bridged, my goal here is to highlight one aspect of Exo/Astrobiology - the search for evidence of ancient life - in which (from my admittedly myopic perspective) rigorous interdisciplinary science has paid major dividends. Over recent decades, the rules for accepting ancient microfossil-like objects as bona fide Precambrian fossils have come to be well established, namely, that such objects be demonstrably biogenic, and indigenous to and syngenetic with the formation of rocks of known provenance and well-defined Precambrian age. Three new techniques have recently been devised to help answer the question of biogenicity, the most vexing of the criteria to satisfy. Founded on firm understanding of the morphology and physiology of the microorganisms that make up living microbial communities and a large body of data on the maturation of organic matter in geologic settings, for the first time these techniques provide means to correlate 
directly cellular morphology with organic composition in individual ancient microscopic fossils. (1) Ion microprobe analyses have been used to measure the carbon isotopic compositions of single fossils in geologic units $\sim 850, \sim 2,100$, and $\sim 3,500$-Ma-old. ${ }^{1,2}$ (2) Laser-Raman imagery has been used to analyse the molecular compositions of individual cellular fossils and particulate organic matter in twenty-five geologic units ranging from $\sim 400$ - to $\sim 3,500$-Ma-old, including representative specimens from each of the three oldest fossiliferous units now known. ${ }^{3,4,5}$ (3) Atomic force microscopy has been used to reveal the submicron-scale structure of the kerogenous components of single Precambrian microscopic fossils. ${ }^{6}$ Taken together, the results obtained demonstrate that the molecular structure of the fossils analysed varies systematically with metamorphic facies, fidelity of fossil preservation, color of the preserved organic matter, and $\mathrm{H} / \mathrm{C}$ ratios of bulk-sample kerogens - results that not only establish the biogenicity of the kerogenous microscopic fossils analysed but provide new insight into the chemical changes that accompany organic metamorphism. Studies such as these require expertise in disparate disciplines and their particular techniques - microbiology and micropaleontology; organic chemistry and biochemistry; stable isotopic and organic geochemistry; secondary ion mass spectrometry, laser-Raman spectroscopy, and atomic force microscopy. Given this, the reality that Exo/Astrobiologic science is inherently multidisciplinary, what can be done to help our field? Three suggestions: (1) Institute month-long summer workshops for senior workers new to the field, taught by experienced 'old-timers' and designed to produce publishable results. (2) Establish a pan-European facility properly equipped (for laser-Raman imagery, ion microprobe spectrometry, confocal-optical, electron, and atomic force microscopy, etc.) and specifically dedicated to an integrated chemical-morphological search for ancient evidence of life. And, over the long term, (3) encourage university level-multidisciplinary education in the sciences. ${ }^{7}$

\section{References}

${ }^{1}$ House, C. H., Schopf, J. W., McKeegan, K. D., Coath, C. D., Harrison, T. M., and Stetter, K. O., Carbon isotopic composition of individual Precambrian microfossils. Geology 28 (2000), 707-710.

${ }^{2}$ Ueno, Y., Isozaki, Y., Yuimoto, H., and Maruyama, S., Carbon isotopic signatures of individual Archean microfossils from Western Australia. Internat. Geol. Rev. 43 (2001), 196-212.

${ }^{3}$ Kudryavtsev, A. B., Schopf, J. W., Agresti, D. G., and Wdowiak, T. J., In-situ laser-Raman imagery of Precambrian microcopic fossils. Proc. Nat. Acad. Sci. 98 (USA) (2001), 823-826.

${ }^{4}$ Schopf, J. W., Kudryavtsev, A. B., Agresti, D. G., Wdowiak, T. J., and Czaja, A. D., Laser-Raman imagery of Earth's earliest fossils. Nature 416 (2002), 73-76

${ }^{5}$ Schopf, J. W., Kudryavtsev, A. B., Agresti, D. G., Czaja, A. D., and Wdowiak, T. J. (in preparation).

${ }^{6}$ Kempe, A., Schopf, J. W., Altermann, W., and Heckl, W. M., Proc. Nat. Acad. Sci. (USA) (in press).

${ }^{7}$ Schopf, J. W. and Hirsch, W. Z., Strategies to foster multidisciplinary teaching and research in a University context. In W. Z. Hirsch and L. E. Weber (eds.), As the Walls of Academia Are Tumbling Down (London: Economica Press) (in press).

\section{Organic Chemistry in Interstellar Medium}

\section{Large organics in space: laboratory measurements of gas phase spectra and Diffuse Interstellar Bands N. Boudin(1), Ph. Bréchignac(2), F. Boulanger(3), B. H. Foing(1) (1) Space Science Department, ESA, European Space Agency, ESA/ESTEC, Noordwijk, The Netherlands, E-mail: nathalie.boudin@rssd.esa.int; (2) Laboratoirede Photophysique Moléculaire, Orsay University, France; (3) Institut d'Astrophysique Spatiale, Orsay University, France}

Polycyclic Aromatic Hydrocarbons (PAHs) are now recognized as an important constituent of the interstellar medium gaseous phase. This family of molecules and in particular their ionic derivatives seem to be the most promising candidates for identification of the Diffuse Interstellar Bands (DIBs). These absorption bands are observed in the visible spectral range in the spectra of stars partially masked by gas cloud. The possible identification of PAHs as molecules responsible for these bands must be done through direct comparison with PAHs spectra recorded in conditions as close as possible to those of the interstellar medium. These low temperature and collision-free conditions can be recreated in the laboratory with supersonic beams. A van der Waals complex PAH$\mathrm{Ar}$ is then ionized by resonant two-photon ionization. The ion absorption of a tunable visible laser photon causes the argon departure, and the detection of this dissociation by a mass spectrometer allows the recording of the PAH cation spectrum. Using this technique, the spectra of phenylacetylene $+-\mathrm{Ar}$ and of acenaphthene $+-\mathrm{Ar}$ could be obtained. The spectral profile is wide and lorentzian, showing an intramolecular dynamics dominated by ultrafast internal conversion. Two observation campaigns with the Télescope Bernard Lyot at the Pic du Midi allowed us to perform a systematic search for this broad band, thanks to specific observational protocole and data processing. Upperlimits of abundance were determined for five PAH cations. Interstellar absorption zones identified in our spectra are of variable width and some of them are very likely the result of a combination due to several species. According to our measurements between 525 and $840 \mathrm{~nm}$, the diffuse interstellar absorption represents $87 \%$ of the interstellar absorption in front of BD + 404220 star

\section{Elemental carbon structures properties and allotropy applied to carbon dust in the Universe} F. Cataldo(1), Y. Keheyan(2)

(1) Soc. Lupi arl, Chemical Research Institute, Via Casilina 1626/A, 00133 Rome, Italy, E-mail: cdcata@flashnet.it; (2) Istituto di Metodologie Chimiche, CNR, Monterotondo Stazione, 00016, Rome, Italy,E-mail:yeghis.keheyan@mlib.cnr.it

Introduction: recently we have started a program on the synthesis and investigation of the chemical structure and properties of carbon allotropes and certain carbonaceous matter. We have tried to frame our work in an interdisciplinary perspective. Here we are summarizing the main results of some selected works published by us on this topic. General aspects about carbon in the Universe: Carbon, together with nitrogen and oxygen is one of the most abundant elements in the universe $^{1}$ after hydrogen and helium. However, it represents only a small fraction of the atoms present in the universe $(0.1 \%$ together with $\mathrm{O}$ and $\mathrm{N})$. The recommended interstellar value for carbon abundance is $225 \pm 50$ carbon atoms per $106 \mathrm{H}$ atoms. ${ }^{1}$ The barionic matter in the interstellar medium accounts for at least $20-30 \%$ of the mass of our galaxy. About 1 to $3 \%$ of the mass of the interstellar medium (ISM) is under the form of microscopic, micron-sized grains ${ }^{3}$ ranging from $10 \mathrm{~m}$ to $10^{-3} \mathrm{~m}$. The majority of the dust consists of carbon and silicates. The formation of carbon in stars and its ejection in the ISM has already been reviewed. ${ }^{3,4}$ The main source of carbon in the ISM is red giants and super giant stars, which have produced about $50 \%$ of the carbon in the galaxy. Novas and supernovas are also important sources whereas planetary nebulas are minor contributor. Carbon dust is formed in the envelopes of carbon-rich late type stars. In the carbon-rich giants, the elemental $\mathrm{C} / \mathrm{O}$ ratio in the mass outflow is greater than 1 and essentially all oxygen is bound to the stable $\mathrm{CO}$ molecule. Therefore the remainder of the carbon is present in the form of acetylene, polyynes, cyanopolyynes and sulfurterminated allenic carbon chains. They are considered precursor in the formation of polynuclear aromatic hydrocarbons (PAHs) and carbon dust. ${ }^{2-6}$ The most exciting aspect for a carbon scientist is the understanding that the formation of carbonaceous material in the outflows of evolved C-rich stars is assumed to be similar to that of soot formation in hydrocarbon flames. ${ }^{4,6}$ Thus, as pointed out in our recent review article, ${ }^{7}$ 
it is possible to take and apply our knowledge in the field of soot formation (as developed both in combustion science and in carbon science) to the formation of interstellar carbon dust. This interdisciplinary approach will help us in developing structural and morphological models of carbon dust in the Universe.

More insights into the carbon dust formation mechanism: As already reported in the previous paragraph, we have noticed ${ }^{7}$ that the molecules present in space, namely polyyne chains and rings, polycyclic aromatic hydrocarbons and fullerenes which are the precursors of the formation of interstellar carbon dust have also been detected by in situ mass spectrometry in ordinary flames and in the carbon arc formed between graphite electrodes under special conditions. Thus the mechanism, which leads to soot formation in a flame, should be similar to that leading to carbon dust in space. In fact, the morphology of the carbon black produced industrially by furnace process or by thermal decomposition appears very close to the morphology of carbon dust produced under conditions which should simulate the circumstellar conditions. In both cases curved graphene sheets can be observed together with onion-like particles and defective structures which we have defined fullerene-like structures. The presence of these structures affect the surface chemistry of the carbon dust grains by enhancing the electron affinity in comparison to graphite. The fullerene-like structures in carbon dust may be responsible for instance for the formation of molecular hydrogen from atomic hydrogen by an enhanced surface chemistry mechanism. Carbon allotropy and related topics: In recent years it has been discovered that elemental carbon can occur under amazingly different chemical structures. ${ }^{7,8}$ New carbon allotropes such as carbyne and fullerenes have been added to the traditional diamond and graphite. The 'fullerene-like structure' concept has been formulated and applied to carbon black, although the same concept has been already applied to onion-like carbon, carbon nanotubes. ${ }^{9,10}$ The most interesting step ahead in the field of carbon allotropy is the knowledge that in certain types of elemental carbon the carbon atoms can occur in the same solid matrix under different hybridization states. ${ }^{11,12}$ The consequence of this is the formation of mixed forms of carbon, starting from glassy carbon to diamond-like carbon and carbon black. Furthermore, another valuable concept involves the so-called 'intermediate forms of carbon' like fullerenes, and related fullerenic structures where the hybridization state of the carbon atoms is not an integer but it is comprised between 2 and 3 or cyclopolyynes where the hybridization state is comprised between 1 and 2 . The deviation from an integer number derives from the distortions and tensions introduced in chemical bonds when the carbon atoms are forced to deviate from their usual hybridization state. How all this new knowledge may affect our conception of the structure and morphology of the interstellar and circumstellar carbon dust? To this question we have already tried to give an answer in an earlier work. ${ }^{1}$

Fullerene formation and stability; a comparison with graphite: In a series of works, we have shown that under the action of an intense electromagnetic fields, for instance a laser plume in vacuum, $\mathrm{C}_{60}$ fullerene and its superior homologues can be formed from a variety of carbon substrates ranging from graphite to diamond grains to disordered carbon containing carbyne and diamond-like carbon. ${ }^{13-15}$ Only furnace carbon black was not able to produce fullerenes ${ }^{13}$ while the more graphitized thermal black was able yield fullerenes under laser ablation conditions. As reviewed from literature data, fullerenes can be formed also from very unusual substrates. ${ }^{15} \mathrm{C}_{60}$ and $\mathrm{C}_{70}$ fullerenes are easily oligomerized and photopolymerized in the solid state under the action of photons, ${ }^{16-18}$ but can be re-generated by the action of very intense electromagnetic fields. ${ }^{14}$ Higher fullerenes appear to be much more photostable and less prone to photopolymerization than $\mathrm{C}_{60}$ and $\mathrm{C}_{70}$ fullerenes. In any case, ion bombardment causes the amorphization of fullerenes ${ }^{19}$ and similarly also graphite is radiation damaged and slowly converted into disordered graphite and then amorphous carbon. ${ }^{18,20}$ Optical properties of carbon blacks ${ }^{21}$ : We have also studied the optical properties in the UV-VIS of three carbon blacks having specific surface area from 145 to $7 \mathrm{~m}^{2} / \mathrm{g}$ and produced with furnace or thermal processes. The results have been compared to the optical properties (in the same spectral range) of $\mathrm{C}_{60}$ fullerene photopolymer, to fullerite, i.e. the carbon soot containing fullerenes and to a sample of carbonaceous matter containing carbyne. The scope of the work was to verify if any of the carbonaceous matter studied was able to match the interstellar extinction spectrum which shows a 'bump' at $217.5 \mathrm{~nm}$ and which was originally attributed to interstellar graphitic particles. None of the materials studied has shown a peak at $217.5 \mathrm{~nm}$. All the carbonaceous materials studied showed maxima of absorption from 252 to $267 \mathrm{~nm}$ with the exclusion of $\mathrm{C}_{60}$ photopolymer which has a completely peculiar and different spectrum from all other materials with three maxima at 271, 389 and $510 \mathrm{~nm}$. All the carbon materials studied did not match the $217 \mathrm{~nm}$ peak, hence, cannot be considered the carrier of the interstellar 'bump' but may be present in some circumstellar shells of late type stars, where the peaks at $240-250 \mathrm{~nm}$ have been recorded and attributed to a more ordered and partially graphitized carbonaceous matter. In the discussion, the matrix effect, the particle size, and the clumping of the material studied have been considered as cause of the shift of the peak to a longer wavelength. The carbon black samples have also been extracted with pentane or ethanol and the polycyclic aromatic hydrocarbons (PAH) identified have been discussed in the frame of recent works and in relation to the role that they play in the interstellar medium as potential carriers of the diffuse interstellar bands (DIBS).

\section{Acknowledgements}

We are indebted with ASI, the Italian Space Agency, Viale Liegi, Rome, Italy, for the financial support.

\section{References}

${ }^{1}$ Snow T. P., Witt A. N., Science (1995), 270, 1455.

${ }^{2}$ Henning, Th., Salama, F., Science (1998), 282, 2204.

${ }^{3}$ Hare, J. P., Kroto, H. W., Acc. Chem. Res. (1992), 25, 106.

${ }^{4}$ Henning Th., Chem. Soc. Rev. (1998), 27, 315.

5 Jager, C., Henning, Th., Schlogl, R., Spillecke, O., J. Non-crystalline Solids (1999), 258, 161.

${ }^{6}$ Cherchneff, I., The Molecular Astrophysics of Stars and Galaxies, edited by Harquist T. V., Williams D. A. (Oxford University Press, Oxford, 1998).

${ }^{7}$ Cataldo F., Pontier-Johnson M. A., (2002) Fullerene, Nanotubes and Carbon Nanostructures, $10,1$.

${ }^{8}$ Cataldo F. Kautschuk Gummi Kunststoffe (2001), 54, 22.

${ }^{9}$ Cataldo F. Fullerene Sci. Technol. (2000), 8, 105.

${ }^{10}$ Cataldo F. Carbon (2002), 40, 157.

${ }^{11}$ Cataldo F., Capitani D. Mater. Chem. Phys. (1999), 59, 225.

${ }^{12}$ Heimann R. B., Evsyukov S. E., Koga Y. Carbon (1998), 36, 1654.

${ }^{13}$ Cataldo F., Keheyan Y. Fullerene Sci. Technol. (2001), 9, 553.

${ }^{14}$ Cataldo F., Keheyan Y. Fullerene Nanotubes and Carbon Nanostructures (2002), 10, 99.

${ }^{15}$ Cataldo F., Keheyan Y., submitted for publication.

16 Cataldo F. Polym. Internat. (1999), 48, 143.

${ }^{17}$ Cataldo F. Eur. Polym. J. (2000), 36, 653.

${ }^{18}$ Cataldo F. Fullerene Sci. Technol. (2000), 8, 577.

19 Cataldo F., Baratta G., Strazzulla G. Fullerene Nanotubes and Carbon Nanostructures (2002), 10.

${ }^{20}$ Cataldo F. Carbon (2000), 38, 634.

${ }^{21}$ Cataldo F. Fullerene, Nanotubes and Carbon Nanostructures (2002), 10,155 .

\section{Interstellar organic chemistry \\ S. B. Charnley}

NASA Ames Research Center, Space Science Division, MS 245/3, Moffett Field, CA 94035-1000, USA, E-mail:

charnley@dusty.arc.nasa.gov

Observations and measurements of the chemical composition of primitive Solar System organics, and of dense molecular clouds, should allow the construction of a coherent theoretical picture of the most likely distribution of meteoritic and cometary organic molecules that could seed primitive planets and set the initial conditions for, at least part of, the phase of prebiotic chemical evolution. Recent studies of organic molecule formation in dense molecular clouds will be described. The principal gas phase reaction pathways to complex molecule formation by catalysis on dust grains and by gas phase reactions will be summarized 
and observational searches for new, large, interstellar molecules, will be described. The connection between observed interstellar organics and those detected in comets Hale-Bopp and Hyakutake will also be discussed.

\section{Importances for life of the population III stars B. Corrado Astronomy Department, University of Bologna, Via Ranzani 1, I-40127 Bologna, Italy, E-mail: bartolini@bo.astro.it}

The only elements present in the Universe after the big bang were hydrogen, helium and small quantities of lithium and deuterium. The first stars of the Universe, called population III stars, produced carbon, oxygen, nitrogen and the other heavy elements necessary for life. Due to the lack of heavy elements, the nuclear reactions, usual in the present stars, were unable to efficiently synthetize $\mathrm{C}, \mathrm{N}$ and $\mathrm{O}$. It is now proposed that different nuclear reactions could be activated to produce small quantities of $\mathrm{C}, \mathrm{N}$ able to start the $\mathrm{CNO}$-cycle.

\section{Odin Observations of $\mathrm{H}_{2} \mathrm{O}$ in interstellar clouds} and comets

A. Hjalmarson

Centre for Astrophysics and Space Science at Chalmers University of

Technology, Onsala Space Observatory, SE-439 92 Onsala, Sweden,

E-mail:hjalmar@oso.chalmers.se

This talk is given by the Odin Astronomy Mission Scientist on behalf of the Odin Team (http://www.snsb.se/Odin/Odin.html) On 20 February 2001 the Odin satellite - a new observatory for sub-millimeter wave spectroscopy - was launched from Svobodny in far-eastern Russia. The
Odin project is a Swedish-led, shared (50/50\%) astronomy/aeronomy mission, supported by space agencies and scientists in Canada, Finland, France and Sweden. The astronomy part of the mission is focussed on observations of spectral line emission from interstellar $\mathrm{H}_{2} \mathrm{O}$ and $\mathrm{O}_{2}$. Tenability of the receivers also allows complementary observations of $\mathrm{H}_{2}{ }^{18} \mathrm{O}, \mathrm{CO},{ }^{13} \mathrm{CO}, \mathrm{NH}_{3}$ and $\mathrm{CI}$ (atomic carbon) as well as searches for new interstellar molecules Odin houses a high-precision $1.1 \mathrm{~m}$ diameter telescope equipped with a very flexible cryogenic sub-mm receiver package. Three tuneable Schottky mixers cover the frequency range 541$581 \mathrm{GHz}$ and a fourth Schottky mixer covers the band 486-504 GHz. A $119 \mathrm{GHz}$ fix-tuned HEMT preamplifier allows very sensitive searches for interstellar $\mathrm{O}_{2}$. All receivers are operated in single-sideband mode. Any combination of four, three, or two receivers (depending upon the available power and mode of operation) can be used in combination with two auto-correlation spectrometers (bandwidth range: $100-800 \mathrm{MHz}$; resolution range: $0.125-1 \mathrm{MHz}$ ) and an acoustic-optical spectrometer, AOS (bandwidth: $1000 \mathrm{MHz}$; resolution $1 \mathrm{MHz}$ ). The Odin antenna beam-widths are about $2^{\prime}$ and $9^{\prime}$ at 557 and $119 \mathrm{GHz}$. This talk will highlight new astronomy results from the first year of Odin operation sensitive observations of $\mathrm{H}_{2} \mathrm{O}$ and $\mathrm{H}_{2}{ }^{18} \mathrm{O}$ in a number of comets and in interstellar molecular clouds. The intriguing non-detection (so far) of $\mathrm{O}_{2}$ also will be discussed. Odin is a Swedish-led satellite project funded jointly by the Swedish National Space Board (SNSB), the Canadian Space Agency (CSA), the National Technology Agency of Finland (Tekes) and the Centre National d'Etudes Spatiales (CNES, France). The Swedish Space Corporation was the prime contractor for development and launch of Odin and is responsible for the operation of the satellite.

\section{Chemistry of the Origin of Life}

\section{Molecular evolution in the primitive Earth: Fractal dimension of archea RNAs and viroid sequences compared to computer-generated random sequences} G. Bianciardi

Department di Patologia Umana e Oncologia, University of Siena, Via delle Scotte 6, 53100 Siena, Italy,E-mail: gbianciardi@unisi.it A possible scenario for the origin of life needs an informational genetic polymer and an efficient prebiotic synthetic route to the component monomers. Nothing is known about the way(s) from which life is born, and plausible pathways of prebiotic evolution remain obscure; however, in that context, RNA is considered the oldest known informational genetic polymer. To examine the general properties of ancient genetic codes, we have evaluated the Manhattan and Euclidean fractal dimensions (Dm, De) of tRNAs in Archaea (the oldest known RNAs in the most ancient living microorganisms) and in viroid nucleotide sequence data (molecular fossils of the RNA world, according to some authors), comparing to the values from mRNA of bacteria, mitochondria and metazoa and to the ones from computer-generated random sequences. Here, fractal dimension was used as a tool to measure complexity, where the most complex sequence results to be the random sequences (with $\mathrm{D} \gg 1$ ). Our results show that Dm and De values of ancient informational polymers are statistically higher than the ones from bacteria, mitochondria and metazoa and lower than the ones of randomly generated sequences. These results suggest that in the primitive Earth informational polymers originated from slightly edited random strings and that during biologic evolution the distance from randomness increased.

\section{Vibrational energy transfer in the FO FI ATP synthase L. Cruzeiro-Hansson CCMAR and FCT, University of Algarve, Campus de Gambelas, 8000 Faro, X 7417, Portugal,E-mail: lhansson@ualg.pt}

Proteins act as the machines of life, they drive essentially all the physical and chemical processes that go on in living cells: they catalyse reactions, pass signals and provide basic structure. Although they are 100 million times smaller than man-made machines they can perform similar tasks, such as transport molecules from one part of a cell to another and act as motors. The way they function is still very obscure but it is known that they perform their functions by going from one conformation into another. The possibility that vibrational energy transfer is a step in protein function was proposed in the early 70 s in connection with a mechanism for muscle contraction. ${ }^{1}$ Computer simulations show that vibrational energy transfer is indeed a very robust way of transferring energy from the active site of a protein to other regions where the energy is used for work. ${ }^{2}$ Recent experiments lead to a lifetime of $15 \mathrm{ps}$ for such a vibration in myoglobin, ${ }^{3}$ two orders of magnitude larger than most people thought. Still missing are experiments to demonstrate directly the participation of this process in protein function. In this talk the results of such an experiment on the F0 F1 ATP synthase, the protein that synthesizes ATP, will be described.

\section{References}

1 A. S. Davydov, J. Theor. Biol. 38: 559-569 (1973)

${ }^{2}$ L. Cruzeiro-Hansson and S. Takeno, Phys. Rev. E 56: 894-906 (1997).

3 A. Xie, L. V. D. Meer and R. H. Austin, Phys. Rev. Lett. 84: 5435$5438(2000)$.

\section{The rule of enantiomorphic crystals in origin of chirality on Earth and other planets E. I. Klabunovskii \\ N. D. Zelinskii Institute of Organic Chemistry, Russian Academy of Sciences, Laboratory of Asymmetric Catalysis 119991 Moscow, 47 Leninskii prospect, Russia,E-mail: klab@cacr.ioc.ac.ru}

It is well known that enantiomorphic crystals can serve as asymmetric adsorbents, as catalysts and as carriers for catalysts. Therefore, many authors claimed on a possible role of such crystals in enantiomeric pure form as the source of chirality and even homochirality of organic molecules in nature. To realize the idea of Bernal there were a lot of attempts to confirm the asymmetric adsorption of organic compounds on different types of clay minerals - based on natural alum silicates, but all these attempts were unsuccessful despite using many methods of enantiomeric analysis, and enantiomorphic feature of clay minerals 
remained not proved yet. Only in the case of crystals of optically active quartz the chiral character of crystals was experimentally proved in the processes of asymmetric separation of enantiomers of racemic organic compounds and in asymmetric catalysis on metal supported on quartz crystals. But in the both cases effects were very feeble. ${ }^{1-3}$ Only recently the theoretical model of asymmetric auto catalysis ${ }^{4}$ was realized in an experiment which could observe the almost perfect optically active product in asymmetric addition reaction at $0{ }^{\circ} \mathrm{C}$ of pyrimidyl-carbaldehyde with dialkylzinc into pyrimidyl-alkanol in the presence of crystals of optically active quartz. ${ }^{5}$ Independence of the sign of optical activity of quartz, d- or 1, the (S)- or (R)-absolute configurations of product were obtained. The same very high effects in this reaction were obtained and in the presence of $\mathrm{d}$ - or 1-ionic crystals of Sodium chlorate. ${ }^{6}$ These processes can be explained in terms of amplification of very small chiral effects created by intermediate reaction product involving chiral component quartz crystals, playing the role of catalyst of process. Thus, the results have a twofold significance: first, as a practical method to amplify the small chiral effects in organic reactions, and, second, as a possible way to discover the chirality of minerals to be used as chiral catalysts, or carriers and to search for the possibility of the origin of chirality on Earth, other planets and moons, where enantiomorphic minerals like quartz can exist. In the case of quartz $\mathrm{Soai}^{5}$ claimed: 'results provide chemical evidence that chiral quartz may be involved in the origin of the chirality of organic molecules through catalytic asymmetric synthesis'. Of course, this statement is true only in the relation of the origin of chirality rather then homochirality because it is known that amounts of d- and l-quartz crystals in all locations on Earth investigated up to now are equal to each other. ${ }^{7,8}$ Therefore, calculations of the parity violation energy difference that can give the prevalence to l-quartz crystal as source of homochirality on Earth proved to be wrong. ${ }^{9}$

References

${ }^{1}$ Schwab G. M.: Naturwissenschaften, 20, pp. 363-364 (1932).

${ }^{2}$ Klabunovskii E. I.: Asymmetrische Synthese, VEB. Berlin., pp. 201 (1963).

${ }^{3}$ Bonner W. A.: in: Physical origin of homochirality in life, Amer. Inst Phys. Conference Proceedings 379 (ed. D. B. Cline) Santa Monica CA. AIP Press. Woodbury, NY, pp. 17-49 (1996).

${ }^{4}$ Frank F. C.: Biochim. Biophys. Acta, 11, pp. 459 (1953).

${ }^{5}$ Soai K. J.: Amer. Chem. Soc., 121, pp. 11235-11236 (1999).

${ }^{6}$ Sato I.: Angew. Chem. Int. Ed., 38, pp. 1510-1512 (2000).

${ }^{7}$ Klabunovskii E. I. and W. Thiemann: Origin Life Evol. Biosphere, 30, pp. 431-434 (2000).

${ }^{8}$ Klabunovskii E. I.: Astrobiology, 1, pp. 127-131 (2001).

9 MacDermott A. J.: Physical origin of homochirality in life, Amer. Inst Phys. Conference, Proceedings 379 (ed. D. B. Cline) Santa Monica, CA. AIP Press, Woodbury, NY (1995).

\section{On the concept of parity in nucleotide alphabets and implications for the possible existence of alternative alphabets \\ D. A. MacDónaill \\ Department of Chemistry, University of Dublin, Trinity College, Dublin 2, Ireland, E-mail: dmcdonll@tcd.ie}

The potential bifurcation of hypothetical biochemistries based on the D- and L-stereochemistries of amino-acids and sugars is self-evident. In this paper the case for a further potential bifurcation based on nucleotide parity is outlined; it is argued that the recognition features of nucleotides possess parity, in which the purine/pyrimidine feature is related to the hydrogen acceptor/donor pattern as a parity bit. Viable alphabets, for reasons of replication fidelity, must be composed from nucleotides of like-parity only. Thus a further bifurcation of life into systems employing even-parity and odd-parity alphabets seems possible. The successful replication of nucleotides other than the familiar A, C, G and $\mathrm{T} / \mathrm{U}$ has been demonstrated in a number of studies, ${ }^{1-3}$ and it seems reasonable to suggest that under different chemical conditions different nucleotide alphabets might emerge. Investigations into nucleotide alphabet composition tend to focus on physicochemical and related issues. This paper, recognizing that nucleotide replication is ultimately an information transmission phenomenon, considers the role informatics might play in determining the composition of an emerging replicating alphabet. The recognition features of nucleotides are mapped to 4-digit binary numbers by (arbitrarily) interpreting a lone-pair as 0 , and a hydrogen as 1 , and similarly equating (again arbitrarily) a purine with 0 and a pyrimidine with 1 . Thus, for example, $\mathrm{C}$ may be interpreted as $(100,1)$, while $\mathrm{G}$ corresponds to $(011,0)$. An analysis based on errorcoding theory leads to the following conclusions: (1) Optimal alphabets are those in which the purine/pyrimidine feature is related to the acceptor/donor pattern as a parity bit. In such alphabets all nucleotides, numerically interpreted, have the same parity, a property which in information transmission confers simple but effective error-resistant properties. (2) Of the 16 possible nucleotides corresponding to 4-bit numbers, viable alphabets must be composed from up to eight evenparity nucleotides, or eight odd parity nucleotides. However, no alphabet can contain more than eight letters. (3) The natural alphabet of A, C, G and T/U obeys the parity rule, and is a subset of the even-parity set, constrained to its present size and composition by a combination of error-coding and chemical factors. Alternative, possibly larger, evenparity alphabets might exist but only in biochemistries where the chemical difficulties, such as the automatic instability of iso-G, is overcome. The paper explores these issues in detail, considering the combined effects of informatics and chemical constraints on the composition of the natural and alternative alphabets.

References

${ }^{1}$ C. Y. Switzer, S. E. Moroney and S. A. Benner, J. Am. Chem. Soc., 111 (1989), 8322.

2 S. Moran, R. Ren, S. Rumney and E. T. Kool, J. Am. Chem. Soc., 119 (1997), 2056.

${ }^{3}$ J. A. Picirilli, T. Krauch, S. E. Moroney and S. A. Benner, Nature, 33 (1990), 343.

\section{The RNA world: hypothesis, cellular facts and experimental results \\ M.-C Maurel}

Institut Jacques-Monod, University Paris 6, Biochimie de l' Évolution et Adaptabilité Moléculaire, Tour 43, 2 place Jussieu, 75251 Paris, Cedex, France,E-mail:maurel@ijm.jussieu.fr

RNA in extreme environment - in favour of RNA world hypothesis: The fact that this macromolecule alone can stock genetic information and perform catalysis. Against: RNA is temperature sensitive (if early life was thermophile). However we have shown that RNA is more thermostable in the presence of salt, that is to say in halophilic extreme conditions. Salt and water are tightly linked and there is evidence that primitive oceans on earth were highly saline. Salt is also abundant on planets such as Mars and Europa. If life arose in thermophilic environments, the RNA world hypothesis would have to include mechanisms to stabilize RNA in these conditions. We show the integrity of tRNA at high temperature and high salt concentrations. Stability was also shown by measuring the activity of the molecule. Our results suggest that a hyper saline environment is favorable for the preservation of RNA. We are now selecting RNA well-conserved at high temperature and high salt capable of survival more than sixty-five hours with two molar salts. 


\section{Space Exposure Experiments ISS/EXPOSE and BIOPAN}

\section{BIOPAN-4 on FOTON-MI : the fifth flight of the ESA exposure facility}

P. Baglioni(1), R. Demets(1), W. Schulte(2)

(1) European Space Agency, ESA/ESTEC, The Netherlands,

(2) Kayser-Threde GmbH Wolfratshauser Strasse 48 D-81379 Munich, Germany,E-mail:sw@kayser-threde.de

The ESA BIOPAN facility is being prepared for its fifth flight aboard a Russian retrievable satellite; the launch of the spacecraft, named FOTON-M1, is scheduled for launch in October 2002. The facility was designed in the early 1990s as an exposure platform for biological samples like microbes, plant seeds and amino acids, to the harsh space conditions in low earth orbit where almost untenanted solar UV radiation, cosmic radiation and extreme temperatures predominate. After a first technology demonstration flight, three orbital flights with biological experiments have been carried out, each of them successful. For the upcoming flight, an unprecedented number of nine experiments, laiddown in fourteen different sample assemblies, will be accommodated in the facility. This shows the increasing interest in the scientific community for this experiment platform and the versatility of BIOPAN as a multiuser facility. In BIOPAN the experiment assemblies are mounted on temperature controlled experiment carrier plates within a cylindrically shaped experiment container. The plates are installed in the container bottom and in the deployable lid. Temperatures and incident solar UV and visible solar light are monitored by the facility throughout the flight. The nine experiments that will be carried on the fifth flight, named BIOPAN-4, resort under different scientific fields : - Exobiology: LICHENS: measurement of the survival rate of lichens, exposed to the harsh space environment. MARSTOX: investigation of the potential toxic effects of Martian soil plus solar UV on microbial spores. ORGANICS: quantification of the integrity of different types of organic compounds exposed to solar UV. PERMAFROST: measurement of the effects of the space environment on the survival rate of microbial life in permafrost soil. - Radiation biology: PHOTO: the effect of cosmic rays on the photosynthetic activity of unicellular algae. YEAST: quantification of the survival rate of yeast cells exposed to soft cosmic rays (electrons, protons) - Environmental science/radiation dosimetry: LETVAR: comparison of the protective effects of four different types of radiation shielding materials. RADO: a multi-facetted radiationdosimetry study using different detector types for measuring soft and hard rays, penetration depths, LET values, as well as the shielding characteristics of charged glass.

R3D-B: measurements, with time resolution, of the irradiance of solar light (divided into four wavelength ranges) and hard cosmic rays. The only existing BIOPAN flight unit, already flown twice in the past, has been extensively refurbished to fly with FOTON-M1. In addition to a new structure and a new heat shield, modifications have been implemented in order to cope with the new experiments lay-out: the new experiments packages, larger in size and mass, have required a partial redesign of the experiment carrier plates, aimed to increase their structural strength and to allow the nine experiments accommodation. The operational temperature range of the facility has been extended at a lower limit: lid mounted experiments can now be passively exposed to temperature peaks as low as $-40{ }^{\circ} \mathrm{C}$, depending on the actual ambient thermal conditions in orbit. Prior to flight BIOPAN is subjected to a sequence of functional/environmental tests - almost completed at this point in time - and to a full mechanical/electrical interface test with the FOTON-M1 satellite in the TsSKB premises, in Samara, Russia. Experiment integration will take place at ESA/ESTEC two weeks prior to launch.
The Perseus-exobiology experiment on board of MIR F. Boillot(1), A. Chabin(1), C. Buré(1), M. Venet(2), A. Belsky(3), R. Jacquet(1), M. Bertrand-Urbaniak(1), A. Delmas(1), A. Brack(1), B. Barbier(1)

(1) Centre de Biophysique MolMoléculaire CNRS, Rue Charles Sadron, 45071 Orléans Cedex 2, France, E-mail: barbier@ cnrs-orleans.fr; (2) CNES, DSO ED US, BP 22/21, 18 Avenue E. Belin, Toulouse, France; (3) Centre Lasers Intenses et Applications, 351, Cours de la Libération, 33405 Talence Cedex, France

The delivery of extraterrestrial organic molecules may have provided an important contribution to the origin of life on Earth. For instance, the Murchison meteorite contains about 500 organic compounds including nucleic acid bases and 80 amino acids, 8 of them being proteinaceous. Micrometeorites in the $50-100 \mathrm{~m}$ size-range probably constituted the main fraction of the extraterrestrial organic material delivered to the Earth. ${ }^{1}$ In order to study photochemical behavior of amino acids and peptides when carried by cosmic grains in the solar system, samples of these molecules were displayed outside the MIR station to study their chemical behavior. We studied their comportment towards the space environment, especially solar UV radiation. During the Perseus Exobiology experiment, samples of leucine (Leu), - methyl leucine (MeLeu), leucine diketopiperazine, the cyclic dipeptide, (LeuDKP) and trileucine thioethyl ester (Leu3SEt) were exposed for ninety-seven days as $0.5 \mathrm{~m}$ thick solid films to space conditions. They were selected in order to study their resistance to racemization i.e. the loss of one-handedness (Leu and MeLeu), the chemical stability of the peptide bond (DKP and Leu3SEt) and a possible photochemically induced polymerization (Leu3SEt). The samples were exposed both in free form and associated with $5 \mathrm{~m}$ thick films of powdered minerals to mimic the micrometeorites, i.e. a montmorillonite clay, basalt and Allende meteorite. In the case of Leu DKP and Leu3Set, different thicknesses of meteoritic deposits were used to determine the protection threshold. In the absence of mineral protection, up to $50 \%$ of the amino acids were destroyed. The two peptides were shown to partially sublimate as confirmed by ground-based experiments. The amount of degradations due only to UV radiation reached $20 \%$ and $13.5 \%$ for Leu3SEt and DKP, respectively. For the amino acids, the main photochemical degradation process has been shown to be decarboxylation, whereas decarbonylation was observed for peptides. The photolytic by-products were studied by infrared spectroscopy and mass spectrometry. No polymerization of Leu3SEt and no racemization of Leu and MeLeu could be observed. For $5 \mathrm{~m}$ films, meteorite powder provided the best protection whereas montmorillonite clay was the least protective. This result may be explained by the difference of absorption of the minerals in the vacuum UV ( $<190 \mathrm{~nm})$, which corresponds to the domain where the samples absorb and where photons are the most energetic. The protection threshold of the meteoritic powder was determined to be about $5 \mathrm{~m}$. This mission has allowed a better understanding of the conditions required to protect the building blocks of life during their space journey. The photochemical processes involved in the solid state have also been studied. ${ }^{2}$ Of much longer duration than the two previous BIOPAN experiments, ${ }^{3}$ the Perseus-Exobiology experiment constituted an excellent transition for the exposure experiments planned onboard the ISS.

\section{References}

${ }^{1}$ Maurette M. Origins Life Evol. Biosphere (1998), 28, 385-412.

${ }^{2}$ F. Boillot, A. Chabin, C. Buré, M. Venet, A. Belsky, R. Jacquet, M. Bertrand-Urbaniak, A. Delmas, A. Brack and B. Barbier. Origins Life Evol. Biosphere (2002) (accepted).

${ }^{3}$ Barbier B., Bertrand M., Boillot F., Chabin A., Chaput D., Henin O., Brack A. Biol. Sci. Space (1998), 12, 92-95. 


\section{Photochemical formation of oligopeptides in presence of extraterrestrial minerals \\ N. Borisovna, N. B. Gontareva}

Institute of Cytology, Laboratory of Astrobiology, Russian Academy of Sciences, Tikhoretsky pr., 4194064 St Petersburg, Russia,

E-mail:ngontar@mail.cytspb.rssi.ru

The formation of organic molecules in interstellar medium and their further safe transportation to the Earth was tested in the laboratory conditions. Incorporating recent results obtained during several space flights, as well as laboratory simulation experiments, this research focusses on two irradiation types as possible sources of energy and three minerals of extraterrestrial origin. Meteorites Allende and Murchison are considered as the delivery device for external organics. Dry films containing aminoacid mixture Gly were exposed to vacuum ultraviolet radiation $145 \mathrm{~nm}$ (VUV) and ultraviolet radiation $(254 \mathrm{~nm})$ both in the presence and in the absence of lunar soil, meteorite Allende and Murchison powder. The possibility of oligopeptide synthesis (di- and tripeptides), promoted by both types of ultraviolet irradiation was demonstrated in course of all the experiments. Both lunar soil and meteorites exhibited sufficient protective properties against the destructive action of ultraviolet radiation over the reaction products. It has been shown also that lunar soil is more effective taken as the protective shield for the newly synthesized peptides, if to compare it with meteorites. It was revealed that VUV irradiation acts as a more productive impact factor than UV radiation in these type of reactions. The yield of products in the case of VUV radiation was two orders of magnitude higher than the one in the case of UV radiation. Dipeptides were found to be the major reaction products, while tripeptides were synthesized in a smaller though detectable amount. All the measurements were executed by means of HPLC and mass-spectrometry methods. We discuss the possible role of meteorites functioning as interstellar carriers of basic molecules and providing sufficient protection against destructive action of space radiation. These unique properties of small space bodies could have increased the amount of organic precursors at the stage of the early chemical evolution and could provide plausible explanation of quick life emergence on Earth. The presence of short peptide molecules on the surface of primitive Earth could lead to their further evolution and formation of more complicated organic structures. In general, these results contribute to the panspermia hypothesis and origin of life on Earth and beyond.

\section{The AMINO experiments on EXPOSE}

A. Brack(1), S. Leach(2), M.-C. Maurel(3), F. Raulin(4), B. Barbier(1) (1) Centre de Biophysique Moléculaire, CNRS, Rue Charles Sadron, 45071 Orléans, cedex 2,France,E-mail:barbier@cnrs-orleans.fr : (2) Dpt. Atomes et Molécules en Astrophysique, Observatoire de Paris-Meudon, 92195 Meudon, France; (3) Institute Jacques Monod, Tour 43, 2 place Jussieu 75251 Paris, France; (4) LISA, URA 1404 CNRS, 61, Av. du, Général de Gaulle, 94010 Créteil Cedex, France

The objective of the AMINO experiment is mainly to study space chemistry in the solar system in relationship to the origins of life. The main goal is to support the hypothesis that extraterrestrial life-related compounds, ranging from bioorganic precursors to cells, might have been delivered to the primitive Earth when associated with comets, meteorites or micrometeorites. Another aspect of organic chemistry in the solar system can be studied in Titan's environment. This domain is of primary interest for exobiology, due to the rich organic chemistry that occurs on Titan. The last point concerns the study of some cometary analogues. Earth orbit provides a unique opportunity to study the combined effects of all space parameters. Compared to ground experiments, space is an extended laboratory allowing exposure of samples to all space parameters at the same time, and the irradiation of many samples simultaneously under strictly identical conditions, i.e. microgravity, desiccation and solar radiation. Different materials will be exposed to the space environment. Organic molecules of biological interest such as amino acids, peptides or nucleic acids will be exposed in order to study their stability and reactivity under space conditions. CHN organic oligomers modelling Titan's aerosol, and cometary analogues produced by irradiation of icy mixtures of frozen gases, mimicking typical interstellar ice, will be studied to understand space organic chemistry. Additionally, powders of clay, meteorite or terrestrial rocks will be used to mimic the mineral fraction of micrometeorites or interstellar dust and study their influence as filters or as potential catalysts.

\section{Study of the effect of simulated space environment on nucleoprotein and DNA thin films}

A. Fekete(1), K. Modos(1), M. Hegedus(1), Gy. Rónto(2),

G. Kovacs(2), A. Bérces(2), H. Lammer(3)

(1) Institute of Biophysics and Radiation Biology, Semmelweis University, H-1088 Budapest, Puskin u. 9, Budapest, Hungary, E-mail:fekete@puskin.sote.hu; (2) MTA-SE Research Group for Biophysics, Hungarian Academy of Sciences, Budapest, Hungary; (3) Space Research Institute, Department for Extraterrestrial Physics, Austrian Academy of Sciences, Schmiedlstr. 6, A-8042 Graz, Austria

The PUR experiment (phage and uracil response) is the part of ROSE consortium selected for the first mission on the ISS and its main goal is to examine and quantify the effect of specific space parameters such as VUV, UV radiation, dehydration effects, non-oxidative environments etc. related to space vacuum conditions on nucleic acid models. An improved method for the preparation of DNA thin films (NaDNA and LiDNA) was elaborated and the homogeneity of the films were controlled by spectroscopy and phase contrast microscopy. The complete recovery of the amount of DNA from the thin film was found after dissolution. Electrophoresis of the dissolved DNA indicated an intact DNA structure, while successful PCR amplification an intact function of the molecule, so they are likely candidates for the flight on the EXPOSE facility. A new method for preparation of bacteriophage T7 thin layer has been developed; the quality was controlled by spectroscopy and microscopy. After dissolution almost $90 \%$ of the viability of the phages remained, and the intactness of DNA structure was checked by PCR. DNA and phage thin films were produced in sandwich form as well, and stored in an atmosphere containing a mixture of $\mathrm{N} 2$ and $\mathrm{H} 2$; by quality control of the samples no change has been found. They were tested under simulated space conditions at the IWF space simulation facility in Graz. DNA thin films and bacteriophage T7 thin layers at different r.h. values have been irradiated in sandwich form in normal atmospheric conditions by using a low pressure Mercury lamp and high power $(300 \mathrm{~W})$ Deuterium lamp containing short wavelength $(240 \mathrm{~nm})$ UVC components simulating the extraterrestrial solar radiation. Characteristic change in the absorption spectrum and the decrease of the amount of PCR products have been detected indicating the photodamage of isolated and intraphage DNA.

\section{Spores in artificial meteorites, the experiment SPORES ON EXPOSE}

G. Horneck(1), P. Rettberg(1), C. Panitz(1), G. Reitz(1), B. Hock(2),

A. Lux-Endrich(2), E. Rabbow(3), H. Wänke(4), D.-P. Häder(5),

P. Richter(5), T. Dachev(6), D. Mishev(6)

(1) Institute of Aerospace Medicine, Radiation Biology Section, DLR, inder Höhe, D-51147 Cologne, Germany, E-mail:

gerda.horneck@dlr.de; (2) Technische Universität, München, Germany; (3) RWTH Aachen, Germany; (4) Max Plank Institut für Chemie, Mainz, Germany; (5) Universität Erlangen, Germany; (6) Bulgarian Academy of Sciences, Sofia, Bulgaria

Meteorites may be natural vehicles for transporting resistant life forms, such as bacterial spores, through space. This subject has received increased attention since the discovery of Martian meteorites has provided evidence that rocks can be transported from one planet to another in our solar system. With the experiment SPORES, experimental clues will be provided to the question whether and to what extent meteorite material offers enough protection against the harsh environment of space for spores to survive a long-term stay in space. For this purpose, spores of bacteria, fungi and ferns, which are especially adapted to survive extreme conditions, will be embedded in artificial meteorites and exposed to space by use of the multi-user facility EXPOSE to be attached to the International Space Station in 2004. Carefully controlled parameters of space (e.g., space vacuum, defined wavebands and intensities of solar 
$\mathrm{UV}$, and cosmic radiation) will act on the spore samples which are combined with chemical and/or physical protective agents. In the laminated structure, layers of meteorite material will be sandwiched with biological layers; this design allows us to determine the minimum layer of meteorite material required to protect the spores against the most harmful parameter of space, namely the solar UV radiation. In preparatory ground simulation experiments at the space simulation facilities of DLR, a $1 \mathrm{~mm}$ meteorite layer has been found sufficiently opaque to protect spores of the bacterium Bacillus subtilis against intense UVC radiation. In the intermixed structure, a layer of meteorite powder will be mixed with the biological systems, in order to determine the protective or toxic effects of these substances in space. In addition, physical instruments will provide experimental data for the UV and particle radiation distribution on the top of the meteorite material. After one or two years in space, the viability and impairment of the spores will be analysed in the laboratory using a set of biological and biochemical assays.

\section{The experiment OSMO: Exposure of osmophilic microbes to the space environment \\ R. L. Mancinelli \\ SETI NASA Ames Research Center, Mail Stop 239-4, Moffett Field, CA 94035, USA, E-mail:rmancinelli@mail.arc.nasa.gov}

We have shown during ground simulation experiments and using the European Space Agency's BIOPAN facility flown in earth orbit, that when exposed to the space environment the survival rate of Synechococcus (Nägeli), a halophilic cyanobacterium isolated from the evaporitic gypsum-halite crusts that form along the marine intertidal, and Halorubrum-G, a member of the Halobacteriacea isolated from an evaporitic $\mathrm{NaCl}$ crystal obtained from a salt evaporation pond (grown with and without diphenylamine(DPA) which blocks the synthesis of b-carotene) were higher than all other test organisms except Bacillus spores. These results led to the following hypotheses: 1) carotene precursor pigments protect Haloarcula-G cells from solar UV radiation through UV absorbance; and from vacuum desiccation because they act as radical scavengers. 2) Haloarcula and Synechococcus are protected from solar radiation by $\mathrm{NaCl}$ and gypsum-halite respectively, due to UV absorbance by these salts. 3) Synechococcus contains pigments that protect it from UVA $(1=320-400 \mathrm{~nm}), \mathrm{UVB}(1=280-320 \mathrm{~nm})$ and UVC $(1=1-280 \mathrm{~nm})$. Additionally we hypothesize that 4$)$ the cyanobacterium Lyngbya aestuarii isolated form the marine intertidal will survive exposure to the space environment due to pigment protection and the fact that it can withstand cycles of wetting and drying in nature. The objectives of the work are to: 1) determine the role of the carotenoid pigment and its precursors in solar radiation and vacuum desiccation protection of Haloarcula-G; 2) determine the ability of gypsum-halite and $\mathrm{NaCl}$ crystals to protect osmophilic microbes from solar UVradiation; 3) determine if Synechococcus contains pigments that are important in protecting it from the solar UV radiationflux in space, and 4) determine if Lyngbya aestuarii can survive exposure to the space environment. Results of this study are relevant to the astrobiology goal of the future of life beyond the planet of origin, the evolution of life on early earth (e.g., how did life survive UV radiation prior to the evolution of complex DNA repair mechanisms), future of life on earth and possible life on early Mars, the potential for interplanetary transfer of viable microbes via meteorites and dust particles as well as spacecraft, and the physiology of osmophilic halophiles. Testing for cellular reproduction (i.e., most probable number technique) will be used to determine survivability (except for the filamentous Lyngbya, in which increases in turbidity will be monitored), as will counting the number of live cells using Molecular Probes, Inc., LIVE-DEAD stain. The role of b-carotene and its precursors will be determined in Haloarcula-G by exposing pigmented, non-pigmented cells grown in the presence of diphenylamine (DPA), and non-pigmented cells grown anaerobically, to the space environment. Breaks in the DNA will be determined by radiolabeling nicks (breaks) in the DNA with [a-32P dNTP] followed by translation, and pulse-field gel electrophoresis. To determine the protective capacity of the gypsum-halite for the Synechococcus and the $\mathrm{NaCl}$ for Haloarcula$\mathrm{G}$, a monolayer of cells will be spread across the sample discs, air dried, followed by overlaying 1-4 additional layers of either $2 \mathrm{M} \mathrm{NaCl}$ or $2 \mathrm{M}$ gypsum-halite $\left(\mathrm{CaSO}_{4}+\mathrm{NaCl}\right)$, prior to exposing them to the space environment. Synechococcus pigments will be isolated and screened for their UVA, B and C absorbing capacity to determine their potential for UV protection.

\section{Survivability and protection of bacterial spores in space - the BIOPAN experiments}

\section{P. Rettberg}

Institute of Aerospace Medicine, Radiation Biology, DLR, Linder Höhe, D-51147, Köln, Germany, E-mail:petra.rettberg@dlr.de

For the study of the responses of organisms to space environment and for the future exploration of Mars as another planet which has had the potential for the evolution of life, the survivability of bacterial spores of Bacillus subtilis exposed to different subsets of the extreme environmental parameters in space (vacuum, extraterrestrial solar UV, shielding by protecting materials like artificial meteorites) was investigated. Spores were exposed to space in the BIOPAN facility of the European Space Agency on board Russian Earth-orbiting FOTON satellites (BIOPAN I, III missions). They were exposed either in dry layers without any protecting agent, or mixed with clay, red sandstone, Martian analogue soil or meteorite powder, in dry layers as well as in so-called artificial meteorites, i.e. cubes filled with clay and spores in naturally occurring concentrations. After about two weeks in space, their survival was tested from the number of colony formers. Unprotected spores in layers open to space or behind a quartz window were completely or nearly completely inactivated. The same low survival rate was obtained behind a thin layer of clay acting as an optical filter. The survival rate was increased by five orders of magnitude and more, if the spores in the dry layer were directly mixed with powder of clay, rock or meteorites, and up to $100 \%$ survival was reached in soil mixtures with spores comparable to the natural soil to spore ratio. These data confirm the deleterious effects of extraterrestrial solar UV radiation which, in contrast to the UV radiation reaching the surface of the Earth, contains very rich-energy short wavelengths of the UVB and UVC. Thin layers of clay, rock or meteorite are only successful in UV-shielding if they are in direct contact with the spores. The data suggest that in a scenario of a hypothetical interplanetary transfer of life, small rock ejecta of a few $\mathrm{cm}$ in diameter could be sufficiently large to protect bacterial spores against the intense insolation; however, micron-sized grains, as requested by Panspermia, may not provide sufficient protection for spores to survive. To investigate the biocompatibility of the Martian environment another BIOPAN experiment MARSTOX will be performed during the FOTON M-1 mission. The aim is to use different types of Mars soil analogues and to determine on the one hand their potential toxicity alone or in combination with solar UV (phototoxicity), on the other hand their UV protection capability. This experiment will provide new insights into the adaptation to environmental extremes on Earth or other planets which define the principal limits of life and at the same time bear the potential for the evolution and distribution of life.

\section{Biological Samples on ISS-EXPOSE facility for ROSE/PUR experiment}

Gy. Rontó(1), A. Bérces(2), A. Fekete(1), T. Kerérgyártó(2), H. Lammer (3)

(1) Institute for Biophysics and Radiation Biology, Semmelweis University, H-1444, Puskin u. 9, POB 263, Budapest, Hungary, E-mail: ronto@puskin.sote.hu; (2) MTA-SE Research Group for Biophysics, Hungarian Academy of Sciences, Budapest, Hungary; (3) Space Research Institute, Department for Extraterrestrial Physics, Austrian Academy of Sciences, Schmiedlstr. 6 A-8042 Graz, Austria For DNA-based terrestrial solar UV dosimetry bacteriophage T7, isolated phage-DNA and polycrystalline uracil samples have been used The effect of UV radiation was measured by detecting the biologicalstructural consequences of the damage induced by UV photons. Extraterrestrial solar radiation possesses about 3 orders of magnitude higher irradiance, while its spectrum contains short wavelength $(<260 \mathrm{~nm})$ UVC (and VUV) components, unusual in the Earth's conditions. In the 
extraterrestrial conditions the high vacuum excludes the presence of oxygen, thus in space oxidative reactions can occur only depending on the composition of the environment in the vicinity of the sample (e.g. atmosphere inside the sample holder, water content of the sample). The biological samples to be studied will be prepared as thin films of uracil, phage T7 or DNA and they will be closed in a sample holder of sandwich form containing inert atmosphere (Nitrogen). For attenuation of the high solar irradiance filters of 10-, 100-, 1000-fold attenuating capacity are applied, while the effect of shorter wavelengths is investigated with a Deuterium lamp.

\section{Large organics in space: An update of the ORGANICS experiments on BIOPAN and the ISS \\ R. Ruiterkamp}

Sterrewacht, Leiden University, P.O. Box 95132300 RA, Leiden,

The Netherlands,E-mail:ruiterka@strw.leidenuniv.nl

The stellar evolution cycle enriches the Interstellar Medium (ISM) with heavy elements and molecules. Solid and gaseous matter has been integrated in potential planet forming disks. During the formation of our solar system this debris collided with high frequency with the early planets and deposited carbon molecules on the early earth. To investigate the molecular composition of carbon in the Universe is therefore crucial, in order to understand the molecular composition of the primordial Earth. Not only the formation processes play an important role but equally the evolution and destruction of carbonaceous compounds by cosmic rays and UV photons. We present an overview of the current status of the ORGANICS exposure experiments. In these experiments complex organics such as polycyclic aromatic hydrocarbons (PAHs), fullerenes and kerogens will be exposed to space environment. We describe the flight hardware and environmental parameters, scientific rationale and current launch status for the experiments on the BIOPAN module and on the International Space Station (ISS). We also present recent laboratory data in support of these space experiments.
R3D, radiation risk radiometer-dosimeter on BIOPAN and EXPOSE (ISS)

C. Streb(1), P. Richter(1), M. Lebert(1), T. Dachev(2), D.-P. Häder(1) (1) Chair of Plant Ecophysiology, University of Erlangen, Staudtstr.5, D-91058 Erlangen, Germany, E-mail: cstreb@biologie.uni-erlangen.de; (2) Bulgarian Academy of Sciences, Sofia, Bulgaria

The radiation risk radiometer-dosimeter $\mathrm{R} 3 \mathrm{D}$ was designed to measure the radiation climate (solar and ionizing) during the long-term life science experiment SPORES on the ISS (see also G. Horneck, Spores in artificial meteorites, the experiment SPORES on EXPOSE). In October 2002 the device will be tested under space conditions on the BIOPAN facility of the FOTON satellite. During the whole BIOPAN-4 campaign, the solar and ionizating conditions will be monitored by the newly developed measurement device. The R3D is based on two approaches based on valid experience: first the ELDONET system (developed for the European light dosimeter network) used for terrestrial monitoring of solar radiation to investigate among other things ozone depletion in terms of the increase of UV-B radiation, and second the measurement of ionizing radiation in space by Liulin on the MIR space station. After successful miniaturization the prototype will now be exactly calibrated on the FOTON flight. The actual doses of solar irradiation is measured in different wavelength bands; UV-A (315-400 nm), UV-B (280$315 \mathrm{~nm}$ ), UV-C $(<280 \mathrm{~nm}$ ) and PAR (Photosynthetic active radiation, 400-700 nm) channels with a $\mathrm{SiC}$ photodiode based dosimeter provide data and the cumulative dose is determined. The LET spectra of ionizing radiation are acquired using a 256 channels spectrometer dosimeter. The analysis of the spectra will give as well the total dose in $\mathrm{mGy} / \mathrm{h}$ and the particle flux in particle $/ \mathrm{cm}^{2}$, the two space radiation parameters. Not being a life science experiment itself, the R3D is a valuable data acquisition system, providing biological relevant information at a resolution of one minute, for a sample of other life science experiments located on external platforms of space vehicles. After the exposure the effects of the space radiation climate (e.g. the viability of spores) will be analysed in correlation to the data obtained by the R3D instrument.

\section{Planetary Exploration: Laboratory Experiments}

\author{
Laboratory simulations on cosmic materials \\ L. Colangeli, J. R. Brucato, V. Mennella \\ INAF-Osservatorio Astronomico di Capodimonte, Via Moiariello 16, \\ 80131 Napoli - Italy,E-mail: colangeli@na.astro.it
}

Astronomical observations and in situ exploration of Solar System bodies provide information about the chemical and physical properties of materials in different space conditions. Identifying the properties of compounds as a function of environment is a fundamental step to trace their evolution, also in view of biogenic implications. A thorough interpretation of the information provided by in situ and remote observations needs the fundamental contribution of laboratory simulations. Experiments are dedicated to reproduce the properties of species observed in different environments and to simulate the effects of processes active in space (such as thermal annealing, UV irradiation, ion bombardment, gas-solid interactions). The laboratory approach is based on the use of experimental techniques to synthesise organic and refractory 'analogues' of cosmic compounds under well controlled chemical and physical status. The products are characterised in their optical, chemical and structural properties by various analytical techniques. The application of treatments, similar to those active in space, provides information on the reactivity of materials and on the efficiency of treatments. The comparison of laboratory results with data coming from space measurements provides a powerful tool to understand the real nature of cosmic materials and to place constraints on their evolution in space. Recent results obtained by the group of INAF-OAC Naples, will be reported in the present paper.

\section{Facilities for planetary simulation experiments at the IWF in Graz \\ G. Kargl}

Space Research Institute, Department of Extraterrestrial Physics, Austrian Academy of Sciences, Schmiedlstr. 6, A-8042 Graz,

Austria,E-mail: guenter.kargl@oeaw.ac.at

We present an overview on the existing laboratory facilities at the Institut für Weltraumforschung, Graz, and their potential to simulate planetary surface processes and physical properties. There are several areas where experiments have been performed in the past and will be performed in the foreseeable future: (1) Thermal and structural evolution of planetary near surface layers. (2) Determination of soil strength/ cohesion and its variation with depth. (3) Measurement of the electrical conductivity of soil. (4) Providing 'Space-Environments' for exobiological experiments. The instrumentation used for the first kind of experiments is similar to that used for the comet simulation (KOSI) experiments performed by a large research group about 10 years ago in a big space simulator at DLR Köln. Our facility (cryogenic vacuum chamber) is of a much smaller scale, and therefore it is easier to perform series of experiments, where sample composition or other input parameters can be varied in a systematic way. A major device which is currently being installed is a 'solar simulator', which will be used to investigate the so-called 'solid state greenhouse effect' in planetary ices (see abstract by Kaufmann et al.). The second major activity performed in our laboratory are 'penetrometry experiments'. The goal of these experiments is to determine the soil strength of a planetary surface layer 
and its variation with depth. There are two principal methods to measure this parameter: dynamic penetrometry and quasistatic penetrometry. In the former a projectile equipped with an accelerometer is shot into the ground, and the deceleration of the projectile during penetration is measured; in the latter a cylindrical rod with a conical tip is driven into the soil at low speed and the resistance force in response to this penetration is measured by a load cell. Both methods will be applied in near future space missions: Rosetta Lander will shoot an anchor into the ground immediately after landing on the nucleus of comet $\mathrm{P} /$ Wirtanen, and the Mars Netlanders are equipped with rods which will be pushed into the soil after landing in order to ensure good ground contact for the planned seismic measurements. In both cases soil strength measurements using an accelerometer and a load cell, respectively, will be performed. In context of the upcoming Mars Express mission, laboratory studies related to the ground penetrating radar will investigate the electrical conductivity of martian surface analogous material. The principal connection between these experiments is that all investigated parameters are related to each other and depend upon the same structural or rather textural properties. Another aspect of our facility is that we can provide space and planetary environment conditions for exobiological experiments. This has been done already in UV-radiation studies for the expose experiment on the ISS or e.g. in evaluating the survival of halobacteria in martian environment conditions.

\section{Laboratory simulations on complex organics on Mars} I. L. ten Kate

Leiden University, Sterrewacht, P.O. Box 9513, 2300 RA, Leiden,

The Netherlands, E-mail: tenkate@strw.leidenuniv.nl

The search for organic molecules and tracers of life on Mars is the future perspective of several Mars missions. The experimental research programme described here investigates the most abundant organic molecules identified in solar system bodies and beyond, which may have been exogenously delivered to the Martian surface. A vacuum chamber, located at ESTEC, NL, equipped with a solar simulator will be used to collect data on the combined effects of UV photoprocessing, atmospheric conditions and the presence/absence of oxidizing agents on organic molecules. The chamber has a possibility of thermal and pressure control. A window allows the attachment of UV lamps and filters. Samples will be introduced into the chamber on a specially designed tray. Sample trays will be filled with organic molecules embedded in soil analogues, either porous or compact. During the simulation in-situ measurements are taken in the chamber, using a GCMS. Samples will be retrieved with various derivatization techniques. Thereafter the probes are subjected to various analyses, such as HPLC, spectroscopy, GC, LDMS and TOF-SIMS. The results of those simulations are part of a ground support experiment programme for future Mars missions.

\section{Experimental and theoretical investigation of the solid state greenhouse effect \\ E. Kaufmann}

Space Research Institute, Department for Extraterrestrial Physics, Austrian Academy of Sciences, Schmiedlstr. 6, A-8042 Graz, Austria, E-mail: erika.kaufmann@kfunigraz.ac.at

In classical thermophysical models of airless solar system bodies complete opaqueness of the surface material has been assumed. This means that the insolation is absorbed at the immediate surface. However, if the surface consists of particles which are neither dark nor optically thick, the assumption of absorption at the surface is no longer valid. In the case of a medium which is optically thin in the visible and opaque in the thermal infrared (e.g. water ice, $\mathrm{CO}_{2}$-ice, etc), a deposition of energy below the surface occurs and therefore the average temperature of interior layers should be higher than the average surface temperature. This subsurface heating process is the so-called 'solid-state greenhouse effect'. Recently a new project was started at Space Research Institute to reach a better understanding of the phenomenon described above. In our research we will concentrate on the thermal and optical properties of icy areas on Earth, the Mars polar caps, Jupiter's satellite Europa, and comets. Three mutually supplementing methods will be applied: (1) laboratory measurements of different sample compositions under space conditions, similar to the 'KOSI' experiments, (2) field tests, e.g. absorption measurements on terrestrial analogues like alpine glaciers and absorption measurements on Icelandic glaciers for the investigation of the 'super greenhouse effect', and (3) theoretical modelling. The instrumentation that will be used for the experimental investigation is presented and some results from a theoretical model are shown.

\section{Survival, activity and diversity of microbial communities in a Mars simulation environment} B. Aa. Lomstein(1), A. A. Hansen(1), J. Merrison(2), P. Nornberg(3), K. W. Finster (1)

(1) Department of Microbial Ecology, Institute of Biological Sciences University of Aarhus, Building 540, Ny Munkegade, DK-8000 Aarhus C, Denmark, E-mail: bente.lomstein@biology.au.dk; (2) Department of Physics and Astronomy, University of Aarhus, Denmark;

(3) Department of Geology, University of Aarhus, Denmark

The extremely hostile conditions of the Mars environment to living organisms and their remains were simulated in a Mars simulation facility at the University of Aarhus. Natural soil microbial communities were used in the experiments. The main scientific questions that were addressed by the experiments are: (1) is there a potential for life on present day Mars?, (2) which minimum environmental conditions ought to be present on Mars to ensure survival and growth of microorganisms? and (3) may it be possible to find traces of life on present day Mars in the form of biomolecules? Preliminary results from our first exposure experiment will be presented. The microbial community of an iron-rich forest soil was exposed to Martian pressure, temperature, $\mathrm{CO}_{2}$ atmosphere and UV radiation for a time period equivalent to $\sim$ two weeks of Mars UV radiation. At different distances from the soil surface the microbial community and its metabolic activity were characterized before and after incubation in the Mars simulation chamber. Characterization comprised combined cultivation-based and ${ }^{16} \mathrm{~S}$ rDNA-based molecular methods, direct cell counts, viable counts, substrate range, community fingerprinting (DGGE) and potential for microbial respiration of ${ }^{14} \mathrm{C}$ glucose. In addition the soil is characterized with respect to the presence and concentration of biomolecules.

\section{Facilities at the Mars Simulation Laboratory} P. Nørnberg(1), K. Finster(2), F. Folkmann(3), H. P. Gunnlaugsson(3), A. A. Hansen(3), K. Kinch(3), B. Aa. Lomstein(2), J. Merrison(3)

(1) Department of Earth Sciences University of Aarhus, $N y$ Munkegade, bldg. 520, DK-8000 Aarhus C, Denmark, E-mail: geompn@geo.au.dk; (2) Department of Microbial Ecology, Institute of Biological Sciences University of Aarhus, Building 540, Ny Munkegade, DK-8000 Aarhus C, Denmark; (3) Mars Simulation Laboratory Aarhus University, Ny Munkegade, bldg. 520, DK-8000 Aarhus C, Denmark,E-mail:merrison@ifa.au.dk

The Mars Simulation Laboratory was founded with the purpose of studying surface conditions on planet Mars. This is done by an interdisciplinary group of scientists in a number of joint projects. The projects mainly focus on problems related to dust, circulating in the Martian atmosphere and sedimented on the Martian surface. Presently the activity can be categorized into four groups, of which not yet all can present conclusive results: 1. Microbiological survival, activity and community structure under Martian conditions. 2. Heterogenous processes in the Martian atmosphere. Reactive molecules and surfaces. 3. Aerodynamics modelling and magnetic particle capture. Particle adhesion, cohesion and charging. 4. Mars analogue mineralogy. Instrument tests, Mössbauer spectroscopy and XRF. The aim of our studies is to run experiments under conditions as close as possible to the Martian surface conditions. The main instrument for our dust studies is a recirculating wind tunnel, which can be run under Martian gas composition, 
pressure, temperature, UV-radiation and wind speed conditions. In addition to the wind tunnel, the laboratory also has a few smaller chambers for studying soil cores, biological survival, charging etc. The laboratory is run by an interdisciplinary group of scientists under the Faculty of Sciences and has as such access to a wide range of spectroscopic and microscopic methods together with ion-, soft $\mathrm{X}$-ray- and UV beam lines from a storage ring facility. Instruments are to a great extent home built, and an advantage of the laboratory is the access to state of the art workshops. The laboratory facilities will be presented and selected results discussed.

\section{Organics in Comets, Meteoroids and Cosmic Dust}

\section{Organic molecules in comets? Experimental studies and applications to observations \\ H. Cottin, Y. Bénilan, N. Fray, M.-C. Gazeau, F. Raulin, R. Sternberg, C. Szopa \\ LISA, Universite Paris 12, Avenue du General de Gaulle, 94010 Creteil,Cedex, France,E-mail:cottin@lisa.univ-paris12.fr} Today, about twenty molecules have been detected in the gaseous phase of comets. But so far we do not have any direct measurement of the nucleus composition. For more than twenty years, many laboratory experiments have been devoted to the study of the chemistry of cometary and interstellar ices. All of them tend to indicate that compounds more complex than those already detected are also very likely to be present: high molecular weight molecules, polymers, amino acids precursors, or even amino acids themselves. Thus, comets are of prime interest for astrobiology studies. The aim of this presentation is to present a study of the laboratory experiments implemented so far and to build an inventory of organic molecules detected. Doing so, it is interesting to address the following questions: (1) Are some of these complex compounds a key to understanding the history of cometary ices? A molecule could be a signature of such or such process, prevailing during the history of a comet: thermal process, photolysis, charged particles, and catalysis with grains. Are there new experimental developments necessary to answer this question? (2) Will those molecules be detected during the Rosetta mission? The case of chromatographic performances of the COSAC experiment (on board of the Rosetta lander) will illustrate this question. (3) Can we already infer the presence of those complex organics through the observation of extended sources such as $\mathrm{H}_{2} \mathrm{CO}$, $\mathrm{CN}$ and $\mathrm{CO}$ ?

\section{Comets and prebiotic chemistry \\ D. Despois}

Observatoire de Bordeaux, B.P. 8933270 Floirac, France,

E-mail:despois@observ.u-bordeaux.fr

Comets are small icy bodies $(0.1-100 \mathrm{~km})$ left from Solar System formation. They are made of ices and dust, and contain, beside water, small organic molecules, organic refractories and silicates. Some of this material is regularly delivered to the Earth, either directly (collisions) or indirectly (meteors). In both cases, chemical compounds may enrich the Earth atmosphere or reach the ground or the oceans. The amount of (cometary and asteroidal) material delivered to Earth is about $20000 t / y r$ at present and is thought to have been much larger in the past, 100 to 10000 times at least during the first 100 Myrs of Earth history. Cometary material may have played an important, and perhaps crucial role in prebiotic chemistry, bringing first water and carbon, but also readymade reactive small molecules, and possibly more complex species. Comets may also have been a vector of non-racemic mixtures produced in the ISM. We will review here the present knowledge on the chemical composition of comets, and especially the abundances of $\sim 30$ parent molecules believed to be present in cometary ices. These ices may be closely related to interstellar chemistry, where more than 120 species have been identified. Present clues on the composition of organic refractories and silicates will be given. We will discuss some aspects of the delivery process, and confront cometary species to the 'needs' of prebiotic chemistry. The outcome expected from future space missions to comets will be outlined.

\author{
Anatomy of circumstellar dust particles \\ E. K. Jessberger \\ Institut für Planetologie, Westfälische Wilhelms Universität, \\ Wilhelm-Klemm-Str. 10, D-48149 Münster, Germany, E-mail: \\ ekj@uni-muenster.de
}

A major discovery in meteorite research during the last two decades was the isolation and description of circumstellar grains, stardust, in meteorites - like diamonds, graphite and silicon carbides - as well as the identification of their specific stellar sources like AGB stars or supernovae. Isolation and source identification of the typically very small stardust particles - sized at most a few micrometer - was made possible only by isotopic analysis of minute quantities of a few elements. These analyses generally are rather destructive. This is a report on our study to go one step further and to analyse with TOF-SIMS individual particles in three dimensions with a lateral resolution of about $300 \mathrm{~nm}$ and a depth resolution of about $20 \mathrm{~nm}$. Our most important result is that some stardust particles, especially the so-called X-grains that originate from supernovae, are heterogeneous concerning their chemical composition as well as their isotopic composition. The relationships of the variations do not in a simple fashion reflect the standard onion shell model of a 25 -solar-mass supernova. Instead, complex mixing of material from different shells and - maybe more importantly - also the prevention of mixing must have occurred prior to stardust condensation. Our study opens the fascinating prospect to actually look into individual stars from which individual stardust particles originate.

\section{In-situ measurements of cosmic dust \\ I. Mann \\ Institute of Planetology, Westfälische Wilhelms Universität, \\ Wilhelm-Klemm-Str.10, D-48149 Münster, Germany, \\ E-mail:imann@uni-muenster.de}

The study of cosmic dust with in-situ experiments from spacecraft provides data that are complementary to information gained by astronomical observation. Although measurements are impeded by the low statistics of impacting dust particles, the selection of certain sizes and locally occurring dust fluxes, they bear valuable information on those dust particles and dust components that do not reach the Earth. Hence in-situ measurements provide information that augments astronomical observations as well as laboratory-based information. Future in-situ measurements need improved detection methods and should be designed for specific relevant problems of cosmic dust analysis. We describe results from in-situ measurements at comet Halley and in-situ measurements of interstellar dust along with a discussion of the physics of dust measurements and suggest future measurements. We especially discuss the process of impact ionization and its underlying physics, which at the same time may restrict and facilitate future dust experiments.

\section{Atomic Force Microscopy for Cometary Dust W. Riedler(1), K. Torkar(1), H. Jeszenszky(1), J. Romstedt(2), and the MIDAS Team}

(1) Space Research Institute, Department of Experimental Space Research, Austrian Academy of Sciences, Schmiedlstr. 6, A-8042 Graz, Austria,E-mail: riedler@oeaw.ac.at; (2) Research and Scientific Support Department, ESA/ESTEC, The Netherlands

Cometary dust particles probably consist of a complex mixture of organic and inorganic matter together with water ice. Their structure 
results from various agglomeration and ageing processes, most likely resulting in a fine structure composed of different building blocks of silicates, organic materials and ices in the size range from some ten to a few hundred micrometers. In-situ measurements of these particles are the best method to identify the individual components, but have been very rare. The instrument MIDAS (Micro-Imaging Dust Analysis System) aboard the Rosetta Orbiter uses atomic force microscopy (AFM) to produce three-dimensional images of dust grain samples from the coma of $\mathrm{P} /$ Wirtanen at a resolution down to a few nanometers. These images will help to understand the formation processes of cometary material and the role of the organic and inorganic components therein. They also provide statistical information on the dust population, including size distribution, shapes and flux. The paper highlights the capabilities of the instrument, the scientific objectives of the investigation, and the accompanying laboratory work. Although the AFM is a proven laboratory technology, the instrument MIDAS, being its first application in space, has been trimmed to optimize the AFM technique for rough surfaces and for operating in the space environment. Laboratory work with similar instruments on dust analogue materials supports the data interpretation and the further development of the instrument software. The data demonstrate the capabilities of the AFM technique.

\section{Rosetta Lander overview}

S. Ulamec, J. Biele, and the Rosetta Lander Team

Institute for Space Simulation, DLR, Linder Höhe 1, D-51147 Cologne, Germany,E-mail:stephan.ulamec@dlr.de

The Rosetta Lander is part of the ESA cornerstone mission 'Rosetta' to comet $\mathrm{P} /$ Wirtanen and has been provided by an international consortium. It will be ejected from the main spacecraft at a distance of about $3 \mathrm{AU}$ from the sun, descend to the comets surface and investigate the nucleus with a complement of ten scientific instruments. The Flight model of the Lander was delivered to ESA in December 2001, and was environmentally tested together with the Rosetta Orbiter, and is shipped to Kourou early in September. Launch is planned for 13 January 2003, with an ArianeV followed by the commissioning phase. After a cruise period of about nine years and near comet investigation by the Orbiter, landing is foreseen for late 2012. The Lander will be operated (via ESOC) by the Lander Control Centre (LCC) at DLR and the Science Operations and Navigation Centre (SONC) at CNES.

\section{Early Traces of Life and Life in Extreme Terrestrial Environments: Analogues for Extraterrestrial Habitats}

\section{Autocatalysis and self-replication - overview and recent examples \\ G. von Kiedrowski \\ Chair of Bioorganic Chemistry, Ruhr University, NC 2/171, \\ Universitätsstr. 150, D-44780 Bochum, Germany, \\ E-mail:kiedro@ernie.orch.ruhr-uni-bochum.de}

Recent years have seen considerable progress in the development of chemical self-replicating systems. From a general point of view, selfreplicating systems can be defined as autocatalytic reaction systems capable of passing on structural information. The latter requires a certain kind of autocatalyst, namely one which can act as a molecular template in the autocatalytic event. A minimal representation of such an autocatalytic template directed reaction is given by the reaction sequence $\mathrm{A}+\mathrm{B}+\mathrm{C} \leftrightarrow \mathrm{ABC} \Rightarrow \mathrm{C} 2 \leftrightarrow 2 \mathrm{C}$, where $\mathrm{C}$ is a self-complementary template molecule, $\mathrm{A}$ and $\mathrm{B}$ its precursor constituents, and $\mathrm{ABC}$ a termolecular complex which irreversibly reacts to give $\mathrm{C} 2$, a template duplex. The above scheme was applied to achieve self-replication of oligonucleotides, artificial template molecules as well as zipper-peptides. Despite the structural diversity of templates, the rate of autocatalytic synthesis in most of these systems depends on the square-root of the template concentration. Thus, the autocatalytic growth observed here is typically not exponential, but parabolic. The latter type of growth is a consequence of the opposite influences of autocatalysis and product inhibition. We report on the peculiarities of parabolic growth, its sequence selectivity, its temperature response, and its implications for molecular evolution. We show that parabolic growth is also typical for more complex reaction systems in which template autocatalysis occurs with product inhibition. Very recently, first examples for nearly exponential replicators were reported. We present a stepwise exponential replication procedure based on the immobilization of template molecules on surfaces (SPREAD) as well as a recent example for a nearly exponential organic replicator from our lab. Finally we will show surprising evidence from dynamic simulations according to which exponential kinetics are not required for the creation of Darwinian evolvibility of a chemical system, if template replication does not occur in free solution but on surfaces. The significance of this finding with respect to the emergence of the earliest replicators on earth will be discussed.

\section{Hyperthermophilic Archaea as model systems to study origin and evolution of early organisms} M. Moracci(1), F. Carpentieri(1), M. Ciaramella(1), B. CobucciPonzano(1), M. R. De Falco(1), M. De Felice(1), M. Di Giulio(2), B. Di Lauro(1), A. Napoli(1), M. Mazzone(1), G. Perugino(1), F. M. Pisani(1), V. Salerno(1), M. Rossi(1)

(1) Institute of Protein Biochemistry, CNR, Via P. Castellino 111, 80131, Naples, Italy,E-mail: moracci@dafne.ibpe.na.cnr.it;

(2) Istituto di Genetica e Biofisica, CNR, Via G. Marconi 10, 80125, Naples, Italy

Although considerable efforts have been devoted to understanding the emergence of the first living system on Earth, we still do not know when and how life originated. The current preponderance of geological and geochemical evidence favours a warm to hot Earth during the first few hundred million years after accretion. Nowadays, volcanic areas, essentially unchanged for at least $4.3 \mathrm{Ga}$, are populated by hyperthermophilic microorganisms, the majority belonging to the domain Archaea. Furthermore, global phylogenetic models based on conserved RNA and protein sequences also predict, independently from geological evidence, that the early organisms were similar to present-day hyperthermophilic Archaea supporting the hypothesis of a hyperthermophilic origin of life. However, despite these evidences, the hypothesis of a (hyper)thermophilic common ancestor of extant organisms is still under debate; therefore, analysis of the physiological, metabolic and molecular properties of the hyperthermophilic Archaea can contribute to solve this dispute. Furthermore, most Archaea live in almost any environmental niches previously thought of as insurmountable physical and chemical barriers to life (very high or low temperatures, extremes of $\mathrm{pH}$, high pressure, high ionic strength, dessication, exposure to radiations and so on). These findings expanded what we considered the limits of life, stimulating the exobiological research area and increasing the likelihood that life could have evolved in planets considered totally inhospitable. Again, the study of the biology of Archaea can provide useful answers to questions concerning the chemical-physical conditions that are compatible with the mechanisms of abiogenesis and the evolution of early life. In this framework, our group has been involved for a long time in the study of hyperthermophilic Archaea. We faced some crucial questions dealing with the biology of these organisms such as: is the last universal common ancestor (LUCA) a mesophile or a (hyper)thermophile? How it can withstand DNA, RNA and proteins of hyperthermophiles to temperatures as high as the boiling point 
of water? How are Archaea phylogenetically related to the other domains of living organisms regarding DNA replication, transcription and gene organization? We will report on recent advances we obtained on these aspects.

\section{From intraterrestrials to extraterrestrials. Halobacteria from ancient salt deposits - and in outer space?}

H. Stan-Lotter, C. Radax, C. Gruber, A. Legat, M. Pfaffenhümer, H. Wieland, S. Leuko, G. Weidler Institute of Genetics and General Biology, University of Salzburg, Hellbrunnerstr. 34, A-5020, Salzburg, Austria,

E-mail:helga.stan-lotter@sbg.ac.at

Viable halobacteria were isolated from Austrian Permo-Triassic salt sediments; several strains were identified as novel species of the genus Halococcus. ${ }^{1,2}$ Further studies showed that similar isolates were obtained from geographically distant salt deposits in Germany and England. Recently we identified also Halobacterium species in bore cores from Alpine salt deposits, which showed similarity on the genetic level (16S rRNA genes) with isolates from Polish salt mines. The amplification of diagnostic molecules by the polymerase chain reaction (PCR) obviates culturing of microorganisms and has permitted the detection of novel phylogenetic groups in numerous environmental samples. We applied PCR procedures to Alpine Permo-Triassic rock salt and found an unexpected biodiversity in these evaporite environments. ${ }^{3}$ These results support the notion of extreme longevity of some halophilic microorganisms in rock salt. Therefore, halobacteria should be suitable model organisms for exploring the possibility of long-term survival on celestial bodies. The discovery of halite, in some cases also sylvite and water, in meteorites from Mars and from the asteroids, together with the existence of salty brines on the Jovian moon Europa, make it plausible to consider a specific search for extraterrestrial halophilic microorganisms. The search for life on Mars is one goal of ESA for the coming years. Mars and Earth share a common geological history, and evidence exists for liquid water on Mars in the past. If life arose on Mars, traces may still be detectable; evaporitic deposits would be suitable candidates for probing for microbial life. We have started experiments for testing the physico-chemical resistance of halobacterial isolates from ancient salt, including usage of a Mars simulation chamber at the Space Research Institute of the Austrian Academy of Science in Graz.

\section{References}

${ }^{1}$ Stan-Lotter H, McGenity TJ, Legat A, Denner EBM, Glaser K, Stetter KO, Wanner G. Very similar strains of Halococcus salifodinae are found in geographically separated Permo-Triassic salt deposits. Microbiology (1999), 145, 3565-3574.

${ }^{2}$ Stan-Lotter H, Pfaffenhümer M, Legat A, Busse HJ, Radax C, Gruber C. Halococcus dombrowskii sp. nov., an Archaeal Isolate from a Permo-Triassic Alpine Salt deposit. Int. J. System. Evol. Microbiol. (in press).

${ }^{3}$ Radax C, Gruber G, Stan-Lotter H. Novel haloarchaeal ${ }^{16}$ S rRNA gene sequences from Alpine Permo-Triassic rock salt. Extremophiles (2001), 5, 221-228.
Early Earth and early life: an extreme environment and extremophiles? Application in the search for

\section{life on Mars}

F. Westall, A. Brack, B. Barbier, M. Bertrand, A. Chabin

Centre de Biophysique Moléculaire, CNRS, Rue Charles Sadron, 45071 Orléans cedex 02, France, E-mail: westall@cnrs-orleans.fr

Supracrustal rocks from the Early Archaean period from the Barberton (South Africa) and Pilbara (Australia) greenstone belts, dating from the 3.5-3.3 b.y. ago, record a terrestrial environment that was extreme compared to that of the modern Earth: pervasive volcanic and hydrothermal activity, higher surface temperatures $\left(50-80^{\circ} \mathrm{C}\right)$, oceans possibly slightly more acidic and salty, and extremely little $\mathrm{O}_{2}$ in the mainly $\mathrm{CO}_{2}$-rich atmosphere. Moreover, the Earth was mostly water-covered with very little exposed landmass. Early Mars was probably also strongly active volcanically and hydrothermally, although surface temperatures on the planet may generally have been cooler: certainly the planet cooled down very rapidly compared to the Earth, basically freezing between 3.9 and 3.5 b.y. ago. However, there is abundant evidence for the presence of liquid water on the primitive planet, indicating temperatures $>0{ }^{\circ} \mathrm{C}$. The oldest terrestrial body fossils, dating back to $>3.46$ b.y. ago, demonstrate that Early Archaean life consisted of single-celled organisms, morphologically similar to modern bacteria. This means that the earliest fossils record an already relatively evolved stage of life. By combining geological information regarding environmental factors with the fossil evidence, it is possible to arrive at a reasonable hypothesis regarding the type of life represented by the 3.5-3.3 b.y.-old body fossils. All the microbial mats observed, from shallow water to (partially) subaerial environments, occur in intimate association with hydrothermal veins and vents. The organisms that constructed the mats are therefore probably thermophilic. They must also have been anaerobic because there was little free oxygen available. The evolution of (anoxygenic) photosynthesis is suggested by the presence of bacterial mats in shallow water to subaerial habitats, environments strongly affected by sunlight. The lack of $\mathrm{O}_{2}$ in the atmosphere meant that there was no radiation-protective ozone layer, thus the microorganisms had to be able to resist high levels of UV radiation. The consortia living in the microbial mats would have included heterotrophic as well as chemolithotrophic organisms. Mats formed under evaporitic conditions indicate that some early life was at least halotolerant if not halophilic. Thus, early life on Earth was, per se, extreme in comparison with life on Earth today. Microbial life is basically surface-related. Largescale geological processes are generally inconsequential for a form of life whose own microenvironment ranges from micrometers to meters. This implies that differences in the early geological evolution of the Earth and Mars would not have affected the appearance of Martian life, which could have closely resembled that of Early Archaean Earth. However, it is likely that these differences would have influenced the further evolution of life on Mars, since on Earth the appearance of oxygenic photosynthesis, eukaryotes, and multicellular life appears to be related to the increase in atmospheric oxygen, in turn related to plate tectonic burial of organic carbon (as well as oxygenic photosynthesis).

\section{Europe goes to Mars: ESA's Search for Life on the Red Planet}

\author{
Search for traces of live on mars encompasses \\ a large number of disciplines ${ }^{1,2}$ \\ A. E. Brack \\ Centre de Biophysique Moléculaire, CNRS, Rue Charles Sadron, \\ 45071 Orléans, cedex 02, France, E-mail: brack@cnrs-orleans.fr \\ Beagle-2, the exobiology lander of the ESA 2003 Mars Express mission, \\ comprises an integrated suite of instruments to optimize the search for \\ evidence of life on Mars in subsurface and rock interior samples. The \\ package includes instruments to study sample mineralogy (composition, \\ macroscopic, and microscopic structural and textural features), organics
}

(elemental, molecular), oxidation state, and petrology (major and minor element composition). Environmental characteristics will also be measured (atmosphere, radiation budget, temperature, pressure, wind speed and direction, humidity, dust activity). The material will be sampled from protected sites (subsurface and rock interiors) with a mole and a small surface rock grinder and corer mounted on a robotic arm. ${ }^{3}$ The solid sample (soil or rock) will be heated in steps of increasing temperature, each increment being supplied with freshly generated oxygen. Any carbon compound present will burn to give carbon dioxide. The gas generated at each temperature will be analysed by the mass spectrometer. 
The instrument can distinguish between the two stable isotopes of the carbon and quantify the ratio. Other gases can be analysed by the same instrument including methane. The STONE experiment, flown by ESA, was designed to test whether Martian sedimentary material could survive terrestrial atmospheric. A basalt (inflight control), a dolomite (sedimentary rock), and artificial Martian regolith were embedded into the ablative heat shield of FOTON 12, which was launched in September 1999. The collected entry samples have been analysed for their chemistry, mineralogy, and isotopic compositions by a European consortium. Modifications due to atmospheric fall were tested by reference to the untreated samples. The dolomite sample was retrieved intact, although reduced to a depth of about $30 \%$ of its original thickness, suggesting that some Martian sediments could, in part, survive terrestrial atmospheric entry from space. Some kinetic isotopic fractionation accompanied the thermal degradation of the dolomite during re-entry, as evidenced by bulk isotopic measurements on different zones of the residual carbonate. The silica 'fusion crust' from the associated sample holder exhibited a significant degree of isotopic exchange with atmospheric oxygen during re-entry. ${ }^{4}$

\section{References}

${ }^{1}$ Brack, A., Fitton, B., Raulin, F. Exobiology in the Solar System and the search for life on Mars, ESA Special Publication SP (1999), 1231.

${ }^{2}$ Westall, F., Brack, A., Hofmann, B., Horneck, G., Kurat, G., Maxwell, J., Ori, G. G., Pillinger, C., Raulin, F., Thomas, N., Fitton, F., Clancy, P., Prieur, D., Vassaux, D. An ESA study for the search for life on Mars, Planet. Space Sci. (2000), 48, 181-202.

${ }^{3}$ Sims, M. R., Pillinger, C. T., Brack, A. and 26 co-authors. Beagle 2 : a proposed exobiology lander for ESA's 2003 Mars Express Mission, Adv. Space Res. (1999), 23, 11, 1925-1928.

${ }^{4}$ Brack, A., Baglioni, P., Borruat, G., Brandstätter, F., Demets, R., Edwards, H. G. M., Genge, M., Kurat, G., Miller, M. F., Newton, E. M., Pillinger, C. T., Roten, C.-A. and Wsch, E.. Do meteoroids of sedimentary origin survive terrestrial atmospheric entry? The ESA artificial meteorite experiment STONE, Planet. Space Science, in press.

\section{Mars Express and astrobiology}

A. F. Chicarro, R. Trautner

Research and Space Science Department, ESA/ESTEC, Postbus 299, 2200 AG Noordwijk, The Netherlands, E-mail. agustin.chicarro@esa.int,roland.trautner@esa.int

The ESA Mars Express mission, which includes an orbiter spacecraft and the Beagle-2 Lander, will be launched in 2003 by a Soyuz rocket. In addition to a wide variety of scientific objectives concerning the global coverage of the planet for studies of the surface geology and mineralogy, subsurface structure, and atmospheric circulation, composition and escape, as well as the detailed chemical and morphological study of the Beagle-2 landing site, the Mars Express mission will address the issue of astrobiology on Mars both directly and indirectly. Many of the instruments on the orbiter will look for indications of favourable conditions to the existence of life, either at present or during the planets past, and in particular for traces of water in liquid, solid or gaseous form. The science operations of orbiter and lander will be coordinated in specific areas for optimizing the synergy in data obtained, and for maximizing the science output of the mission. The principal objective of the Beagle-2 mission is the detection of extinct and/or extant life, or at least establishing if the conditions at the landing site were suitable for the evolution of life in the past. The instruments on Beagle-2 will try to find traces of life with direct measurements, such as presence of methane $\left(\mathrm{CH}_{4}\right)$ indicative of extant life, and a larger amount of the light $\mathrm{C}_{12}$ isotope compared to the heavier $\mathrm{C}_{13}$, which would indicate extinct life. Among many other objectives, Beagle-2 will also look for the presence of water in Martian soil, rocks and the atmosphere. Overall, the Mars Express mission represents the most comprehensive effort to search for life on Mars since NASA's Viking mission in 1976.

\section{Raman spectroscopy of biogeological materials in extreme environments: from Mars oasis, Antarctica, to planet Mars}

H. G. M. Edwards(1), the late D. D. Wynn-Williams(2)

(1) Department of Chemical and Forensic Sciences, University of Bradford, Bradford BD7 1DP, UK, E-mail:

h.g.m.edwards@bradford.ac.uk; (2)British Antarctic Survey, BAS High Cross, Madingley Road, Cambridge CB3 OET, UK

The cold desert of Antarctica provides an example of the most hostile terrestrial environment in which the survival strategies of organisms such as lichens, mosses and cyanobacteria are tested to the limit. The serious depletion of atmospheric ozone at higher latitudes results in a severe exposure to lower wavelength ultraviolet radiation, particularly that of UVB $(280-320 \mathrm{~nm})$ and UVC $(<260 \mathrm{~nm})$, which necessitates the production of biomolecules for radiation-protection by photosynthetic organisms. The low ambient temperatures, which can reach $-50{ }^{\circ} \mathrm{C}$, and multiple freeze-thaw cycles operating as clouds mask the sun with a frequency approaching 300 times per day, puts additional stress on cryogenic cell protectants and production of water-replacement molecules. The extremely low humidities (approaching zero) also require effective protection against desiccation, which can be exacerbated by strong katabatic winds blowing from the Polar plateau. We have characterized key Raman spectral biomarkers for the identification of protective molecules in Antarctic organisms. An important feature of the work has been the non-destructive analytical capability of the Raman spectroscopic technique, from which the adaptation of the biological organisms to environmental stresses in key locations can be monitored. Bands of key molecules can be compared with an ever-increasing database of Raman spectra for terrestrial materials from analogous habitats in Antarctica. ${ }^{1,2}$ Analysis of potential biomolecules in situ in their natural state within their mineral substrate is a major benefit. Pigments often have functional components in common, such as the UV-absorbing quinone ring of parietin (from lichens) but also different UVabsorptive features as in scytonemin and calycin, with their distinctive Raman spectra. A prototype miniature Confocal-Microscope/RamanSpectrometer (CMaRS) has been developed for a future Mars lander mission and will be evaluated by ourselves in Antarctica. ${ }^{3}$

References

${ }^{1}$ D. D. Wynn-Williams and H. G. M. Edwards, Icarus, 144, 486 (2000).

${ }^{2}$ D. D. Wynn-Williams and H. G. M. Edwards, Planetary Space Sci., 48, 1065 (2000).

${ }^{3}$ D. L. Dickensheets, H. G. M. Edwards, D. D. Wynn-Williams, E. M. Newton, C. Crowder and C. S. Schoen, J. Raman Spectrosc., 31, 633 (2000).

\section{The Mars exobiology multi-user Facility PASTEUR W. Schulte(1), P. Baglioni(2)}

(1) Kayser-Threde GmbH, Wolfratshauser Strasse 48 D-81379 Munich Germany,E-mail: sw@kayser-threde.de; (2) European Space Agency, ESA, ESTEC, 2200 AG Noordwijk, The Netherlands

Since 1996 ESA has promoted scientific and technical studies supported by exobiology science teams and by European space industries in order to investigate the experimental methods and a preliminary concept of a facility capable of searching for traces of extinct and extant life on Mars. Working under the assumption of being installed on a Mars lander (initially identified in the NASA Surveyor-type), a possible instrument package configuration had been identified, and a preliminary facility concept had been established. The facility would include a multi-sample acquisition system, designed to retrieve Mars subsurface samples in different locations from 1.5 to $2 \mathrm{~m}$ of depth, and a sample handling and preparation system, conceived for milling of soil and rock material and for the distribution of the samples to a variety of analytical instruments. The investigation aims at the detection of fossil microorganisms, at the search for organic compounds of biological origin, including biomarkers, and at the analysis of the geochemical and mineralogical context of the analysed samples. The package, named PASTEUR, according to preliminary assessments, could include instruments such as a color optical microscope, Raman and Laser plasma spectroscopy, a mass 
spectrometer, a gas chromatograph, a life marker chip experiment, a subsurface sounder for water detection, and other sensors. It would be complemented by a stereo panoramic camera. In the most plausible scenario the facility would be carried by a rover that is landed on Mars and provides mobility to reach sites of exobiological interest within a few kilometers range. These operations require a very high degree of autonomy with minimal invention from Earth, that anyway should be always possible. Depending on the type of lander-rover that will be selected and on the related available engineering budgets, different instrument compositions and lay-outs are presently being studied, as well as various operational scenarios regarding the number of samples retrieved and the number of measurements performed per sample. At present, a launch date in 2007 is envisaged, while a launch opportunity in 2009 may become a backup solution. ESA is in fact evaluating the feasibility of different mission scenarios, which foresee the use of a rover and of a data relay satellite. A study has been conducted to check the possibility to fit the preliminary facility design with an existing Russian rover - the results indicate the need of an optimization of the exobiology package, both for what concern the miniaturization and the assembly of the instruments and the local management of energy and data resources or the need to develop a new rover to better satisfy all the scientific requirements. The mass of the presently studied facility is estimated to be around $50 \mathrm{~kg}$, its envelope approx. $500 \times 500 \times 370 \mathrm{~mm}$. Available mass/ volume, power, energy and downlink data rates are scarce resources. Depending on the rover's capabilities and payload interfaces the potential of PASTEUR in terms of scientific return is determined and traded in present and future studies. The payload and rover system needs to be equipped with data handling, storage and transmission systems, power supply/generator systems, and thermal conditioning capability. Common systems used for the rover and its payload could contribute to optimize the use of the scarce resources. ESA is about to issue an announcement of opportunities for new instrument proposals and upgraded measurement systems.
ESA's mission to search for signs of life on Mars: ExoMars study and the PASTEUR scientific payload

J. L. Vago, A. Santovincenzo, B. Gardini, and the ExoMars

Study Team

European Space Agency, ESA/ESTEC, Keplerlaan 1, NL-2201 AZ

Noordwijk, The Netherlands, E-mail: jorge.vago@esa.int

ESA has recently completed a study for an exobiology mission to be launched in 2007. Its scientific goal is the search for past and present signs of life on Mars. Life as we know it relies, above all else, upon water. However, water is presently no longer stable on the Martian surface: its temperature and atmospheric pressure are too low. Still, water/ice may lie at some depth underground. Additionally, because of the sterilizing/ degrading effect of the Martian UV radiation spectrum, the search for life indicators, whether for present or for extinct life, should best be conducted below the surface. If approved, ESA's mission will consist of two main elements: a dedicated communications satellite, and a 190-kg rover. The rover will carry the PASTEUR scientific payload. Pasteur is equipped with a multispectral, stereoscopic camera; an electromagnetic subsurface sounder to identify water/ice deposits; a drill capable of reaching a depth of $2 \mathrm{~m}$, and also of collecting samples from within surface rocks; a sample preparation unit, an optical microscope, and a variety of spectroscopic instruments. For the characterization of organic substances, PASTEUR also houses an oxidation sensor, a gas chromatographer/mass spectrometer, and a novel device based on protein assay chip technology. Over its envisioned lifetime, the rover is designed to cover $50-70 \mathrm{~km}$ of ground track over typical Martian terrain. The nominal mission is expected to last up to six months, depending on the landing site's latitude. Operations beyond this period will depend on the amount of dust deposited on the rover's solar panels. This paper addresses the mission, the instruments, and the operations scenario on the Martian surface. Particular attention is devoted to the description of any mission-imposed constraints having an influence on the science output: i.e. for instrument selection and operations, power generation, and landing sites.

\section{Early Traces and Evolution of Life}

\section{The chemical evolution into thunderous clouds V. A. Gusev \\ Sobolev Institute for Mathematics of Siberia Division, Russian Academy of Science, Koptyuga av. 4, Novosibirsk 630090, Russia, E-mail:vgus@math.nsc.ru}

Today it is well known that the first act or the process of the origin of the complex self-reproduction system, which is the cell of microorganisms, could not be in a dark puddle. The media, in which this process is principally not restricted has a great level of its own complexity and in addition a source of energy to synthesis the organic molecules and for keeping all the intermediate structures, from which a living cell is selfconstructed. The work presents a hypothesis about a principal new source of free energy for synthesis of organic molecules from nonorganic oxides and other water-soluble molecules. The media, in which the chemical synthesis may be realized is a water drop flying through thunderous clouds of the primitive Earth atmosphere or other planets. The spherical symmetry of a drop, available at the liquid-gas boundary and the permanent non-equilibrium steady state of the water medium are organizing and ordering factors. First of all required is that electric activity of an atmosphere admit the presence of the wide range of electromagnetic waves. The Langmuir oscillations of water protons are excited in this condition ${ }^{1,2}: \omega_{L}=\left(4 \pi n_{H} / \mathrm{m} \varepsilon\right)^{1 / 2}$, where $\omega_{L}$ is the Langmuir frequency, $n_{H}$ the proton concentration, $\mathrm{m}$ the proton mass and $\varepsilon$ the water dielectric are constant. A planetary atmosphere contains the chemical elements $\mathrm{C}, \mathrm{N}, \mathrm{P}$ and $\mathrm{S}$ as oxides or other water-soluble molecules; that is the second requirement. And of course the planet has liquid water. We will look at the physical conditions on the base start synthesis of organic molecules. It makes sense to assume that proton oscillations have a spherical symmetry, in other words there are periodical proton concentrations and rare faction into the central zone of a water drop. We will look at processes at the drops, whose radius corresponds to average radius of microorganisms $\mathrm{Ro}=0.5 \times 10^{-4} \mathrm{~cm}$. It is assumed that a water drop by micron size represents a prototype of a microorganism cell: within the boundaries of hydrophobic cover exists the solution of organic molecules by physiological concentration. Moreover, a volume of this compartment contained small polymers, among these can be oligo-peptides and oligo-nucleotides as D so $\mathrm{L}$ mono-chirality. The total time of the chemical evolution into a drop is about one second. A 'Soft' source of the energy is an essential moment of this model. Finally, it is very difficult to image that among myriad drops into thunderous clouds which surrounded the primitive Earth with its volcanoes had released huge volumes of carbon oxide, without recognizing the writing process of chemical synthesis based on this model. References

${ }^{1}$ Gusev, V. A. The Electromagnetic Background of the Planets and their Biota. The newsletter of ISSOL the international society for the study of the origin of life, 28 , nos. $1 \& 2$ (spring 2001).

${ }^{2}$ Gusev, V. A. The source of energy for survival and multiplication of heterotroph microorganisms in the absence of organic substratum. Biophysics (Rus.), 46, no. 5, pp. 862-878 (2001).

\section{Terrene meteorites in the Moon}

\section{J. L. Gutierrez}

Department of Applied Physics, Laboratory for Earth and Planetary Exploration Technologies, Universitat Politècnica de Catalunya, Pla de Palau, 18,0800, Barcelona, Spain, E-mail: jordi@fa.upc.es All the rocks predating 3.9 billion years before present (BP) have been destroyed by giant impacts during the Great Bombardment era, tectonic activity and erosion. Nevertheless, there are strong indications of biological activity $3.85 \mathrm{Gyr}$ ago BP. Hence, it seems that life appeared on 
Earth coincidentally with the end of the Great Bombardment. Here we show that pieces of the early Earth, ejected off our planet by impacts during the late stages of Great Bombardment, can probably be found in the Moon. These meteorites could still contain precious information about the origin of life on Earth that our own planet wiped out aeons ago.

\section{Weathering of ultramafic rocks as an energy source for early life forms}

N. G. Holm

Department of Geology and Geochemistry, Stockholm University, SE-10691 Stockholm Sweden, E-mail:nils.holm@geo.su.se Hydrogen formation and abiotic organic synthesis in ultramafic peridotite rock has several implications for our view of the origin of life on Earth and the terrestrial planets. Water in contact with the peridotite is reduced to molecular hydrogen with the concomitant oxidation of $\mathrm{Fe}(\mathrm{II})$. The molecular hydrogen formed may be used directly by chemoautotrophic organisms. At high temperatures the hydrogen may also be combined with $\mathrm{CO}_{2}$ or $\mathrm{CO}$ for the abiotic formation of organic compounds such as hydrocarbons and fatty acids through Fischer Tropsch Type (FTT) synthesis. Such abiotic organic material may be used as an energy source by organoheterotrophic organisms. In addition, weathering of ultramafic rocks provide the most potent environments for the initiation of chemical evolution eventually leading to Darwinian evolution. The chemistry of FTT reactions is likely to promote the concentration and isolation of metabolic systems into separate entities. In contemporary cell membrane lipids, hydrophobicity is provided by hydrocarbon chains of fatty acids with chain lengths ranging from 12 to 20 or more carbons. Linear hydrocarbons with lengths ranging from 16 to 29 carbons have now been isolated in fluids of peridotite-hosted hydrothermal systems of the Mid-Atlantic Ridge. It has previously been difficult to identify plausible sources of long linear carbons chains in the prebiotic environment on Earth. Such chain lengths are relatively abundant in carbonaceous chondrites and it has been hypothesized that amphiphilic substances on Earth may have derived from meteoritic infall. However, the discovery of reactions in hydrothermal systems associated with ultramafic rocks on Earth provides an alternative pathway for the formation of early membranes and the first compartmentalization of life's constituents.

\section{Connectivity nanoscaffolding and the interface between astrobiology and nanotechnology} G. von Kiedrowski

Chair of Bioorganic Chemistry, NC2/171 Ruhr University, Universitätsstr. 15044780 Bochum, Germany,

E-mail: kiedro@ernie.orch.ruhr-uni-bochum.de

In a recent debate about Drexler's vision of self-replicating nanorobots, George Whitesides pointed to a link between nanotechnology and the origin of life: 'Considering the many constraints on the construction and operation of nanomachines, it seems that new systems for building them might ultimately look much like the ancient systems of biology.' Indeed, the ribosome is an example for a nanomachine which may be viewed as a three dimensionally defined array of 51 modular proteins positioned by the rRNA scaffold. Biomimetic approaches towards the 3D-nanoscaffolding of modular functions may be based on trisoligonucleotides, viz. synthetic 3-arm junctions in which the 3 '-ends of three oligonucleotides are connected by a suitable linker. We report on the synthesis of trisoligos and their usage for the noncovalent construction of nanoobjects. Kinetic control-applied by rapid cooling during self-assembly - was found to favor small and defined nanostructures instead of large polymeric networks. Tensegrity and maximal instruction were employed as design principles to generate noncovalent nanoobjects in which both the topology and the geometry is defined. Examples include tetrahedral nanoobjects composed from four trisoligos each bearing three individually defined sequences. The objects were studied by native gel electrophoresis, enzymic digestion, UV-melting curves and distanceprobing using fluorescence resonance energy transfer (FRET). It was demonstrated that the connectivity information in the nanoscaffold junctions can be copied by chemical means. Copying is based on a templated trislinking reaction yielding 5 '-trisoligos when using 3 '-trisoligos as templates. Current studies focus on the reverse copying and the utilization of surface-promoted replication and exponential amplification of DNA analogues (SPREAD) for junction replication. Future work will employ monoconjugates of oligonucleotides and modular functions - such as peptides and tailored thermostable gold cluster labels (RUBiGold) - in the connectivity copying process. Self assembly of the resulting trisfunctionalized junctions should generate constructs, whose tetrahedral nanoscaffdt-fold may encode the 3D-positioning of up to 12 different modules. The potential of such constructs for directed chemical evolution will be discussed.

\section{Mixotricha paradoxa: an archaezoan eukaryotic model organism \\ H. König, M. Wenzel, L. Li, J. Fröhlich}

Institut für Mikrobiologie und Weinforschung, Johannes Gutenberg

Universität, D-55099 Mainz, Germany, E-mail: hkoenig@

mail.uni-mainz.de

Mixotricha paradoxa belongs to the archaezoa, a group of eukaryotes, which branched off at the basis of the eukaryotic tree. In phylogenetic trees archaezoa are located close to the prokaryotic archaea. Therefore, they may serve as model organisms and provide hints for a better understanding of the early evolution of the eukaryotic cell. Mixotricha paradoxa represents a rare example of a movement symbiosis between eukaryotic and prokaryotic microorganisms. The pear-shaped cells are about $500 \mu \mathrm{m}$ long and $250 \mu \mathrm{m}$ in diameter. The surface of Mixotricha paradoxa shows a highly ordered pattern of rod shaped bacteria and in addition it is covered by a dense carpet of spirochetes with the exception of the posterior ingestive zone. The rod shaped bacteria and the spirochetes are attached to regularly arranged protrusions of the cell surface, the so-called brackets. Interestingly, the spirochetes and not the relatively small flagella propel the cells. Based on a $16 \mathrm{~S}$ rDNA sequence analysis after a semi-specific PCR and subsequent fluorescence in situ hybridization applying helper oligonucleotides and a denaturing step of the $16 \mathrm{~S}$ rRNA, three different spirochete clones could be clearly identified on the surface of the protozoal cells. They belonged to the Treponema cluster. The rod shaped bacterium showed highest $16 \mathrm{~S}$ rDNA sequence similarity to Bacteroides-related species. Due to its low phylogenetic relationship to its next relatives in the data base it should represent a so-far undescribed species. Furthermore, bacteria are found in the cytoplasm and in the nucleus. These data show that early eukaryotic cells are a composite association of a eukaryotic cell with living bacteria, but lack classical organelles such as mitochondria.

\section{Spontaneous emergence of catalytic networks in spatially extended systems}

S. C. Manrubia, J. F. Poyatos, J. Pérez-Mercader Centro de Astrobiologia (CSIC-INTA) associated to NASA Astrobiology Institute, Carretera de Ajalvir, km 428850 Torrejon de Ardoz, Madrid, Spain, E-mail: poyatosaj@inta.es

A system of interacting chemical species able to catalize each other's production is studied. We consider a two-dimensional surface where single molecules attach, diffuse, and decay. Interactions are characterized by a highly sparse matrix $c(i, j)$ with $c(i, j)=\{1,0\}$. When $c(i, j)=1$ species $i$ can catalize the production of $j$ following a reaction of the type: $\mathrm{i}+\mathrm{j} \rightarrow \mathrm{i}+2 \mathrm{j}$. Catalytic reactions among molecules appear whenever molecules of species $i$ and $j$ happen to meet in adjacent sites of the lattice and $c(i, j)=1$. Starting with an initially empty surface, new species are added at each time step. The interaction matrix evolves accordingly; coefficients $c(i, j), c(j, i)$ defining the relation of the new species with the rest of molecules present in the surface at that moment are set to one with a very small probability. This situation is thought to describe a surface embedded in a 3 -d bulk of simple reactants which provide the raw material for the synthesis of the more complex molecules bound to the surface. Our main results are (1) robust catalytic cycles emerge after a short transient time; (2) after the transient, a statistically stationary state characterized by high diversity of species (with large population numbers) settles down; (3) there is a continuous turnover of species in the system, though individual species are long-lived; (4) the topology of the 
interaction matrix c(i,j) is self-tuned and selected through the population dynamics, and (5) parasitic invaders are unable to break the high-diversity of the system and its organization due to the explicit introduction of physical space.

\section{Unexpected diversity of heterotrophic prokaryotes living at the highest salt concentrations \\ A. Oren}

The Hebrew University of Jerusalem, Division of Microbial and Molecular Ecology, Institute of Life Sciences and the Moshe Shilo Minerva Center for Marine Biogeochemistry, The Hebrew University, 91904 Jerusalem, Israel, E-mail: orena@shum.cc.huji.ac.il

Halophilic Archaea of the family Halobacteriaceae were until recently considered as the only heterotrophic microorganisms that grow optimally at salt concentrations approaching saturation. Such Archaea abound in hypersaline lakes and in saltern crystallizer ponds for production of solar salt, and they often impart a red color to the brines. It was recently ascertained that extremely halophilic prokaryotes belonging to the domain Bacteria also may occur in large numbers in saltern crystallizer ponds. The first evidence for the presence of an extremely halophilic bacterium, phylogenetically affiliated with the Flavobacterium-Cytophaga group, came from fluoresence in situ hybridization experiments performed in Spanish salterns. ${ }^{1}$ The organism has now been isolated, and was described as Salinibacter ruber. ${ }^{2}$ Salinibacter is an aerobic rod-shaped bacterium that grows optimally between 20 and $30 \%$ salt, and no growth is obtained below $15 \%$. Chloride is specifically required at high concentrations, and cannot be replaced by sulfate or nitrate. The organism is pigmented red, with an absorption maximum at $482 \mathrm{~nm}$ and a shoulder at $506-510 \mathrm{~nm}$. The pigment, salinixanthin, is a novel $\mathrm{C}_{40}$-carotenoid glycoside with the structure (all-E,2'S)-2'hydroxy-1'-[6- $O$-(13-methyl-tetradecanoyl)- $\beta$-D-glycopyranosyloxy]$3^{\prime}, 4^{\prime}$-didehydro- $1^{\prime}, 2^{\prime}$-dihydro- $\beta, \psi$-caroten- 4 -one. ${ }^{3}$ On the basis of HPLC analysis of pigment extracts of biomass collected from the Alicante and Mallorca salterns the Salinibacter pigment was estimated to contribute around $5 \%$ of the total prokaryotic pigment light absorption. ${ }^{4}$ There is a surprising similarity between the physiology of Salinibacter and the aerobic halophilic Archaea of the family Halobacteriaceae. The cells contain molar intracellular concentrations of $\mathrm{KCl}$ to balance the high salt concentration of the medium, as shown by X-ray microanalysis of single cells in the transmission electron microscope. ${ }^{5}$ Using NMR and HPLC no significant concentrations of organic compatible solutes could be detected. The only compounds encountered were glutamate, glycine betaine, and $N \alpha$-acetyl lysine, but these were found at very low concentrations only. ${ }^{5}$ In accordance with its high intracellular ion concentrations, the bulk protein of Salinibacter shares with the members of the Halobacteriaceae a large excess of acidic amino acids and a low content of hydrophobic amino acids. Cytoplasmic enzymes such as isocitrate dehydrogenase, malate dehydrogenase, glutamate dehydrogenase, and the fatty acid synthase complex all function at high salt concentrations, although considerable variation was found in the salt response of individual enzymes. ${ }^{6}$ The discovery of Salinibacter and the elucidation of its properties show that similar modes of adaptation to life at high salt concentrations and low water activities may have evolved independently in phylogenetically greatly distant groups of microorganisms.

\section{References}

1 Antón, J., Rosselló-Mora R., Rodríguez-Valera, F., and Amann, R. Appl. Environ. Microbiol. (2000), 66: 3052-3057.

2 Antón, J., Oren, A., Benlloch, S., Rodríguez-Valera, F., Amann, R., and Rosselló-Mora, R. Int. J. Syst. Evol. Microbiol. (2002), 51: 485-491.

${ }^{3}$ Lutnæs, B. F., Oren, A., and Liaaen-Jensen, S. J. Nat. Prod. (2002) in press.

${ }^{4}$ Oren, A., and Rodríguez-Valera, F. FEMS Microbiol. Ecol. (2001), 36: $123-130$.

${ }^{5}$ Oren, A., Heldal, M., Norland, S., and Galinski, E. A. Extremophiles (2002), submitted for publication.

${ }^{6}$ Oren, A., and Mana, L. Extremophiles (2002), in press.

\section{Primordial formation of ammonia from dinitrogen} on iron sulphide surface

W. Weigand (1), M. Dörr (1), C. Robl (1), G. Kreisel (2), R. Grunert (2), J. Käßbohrer (2), W. Brand (3), R. Werner (3), J. Popp (4),

N. Tarcea (4)

(1) Institute of Inorganic and Analytical Chemistry,

Friedrich-Schiller-Universität Jena, August-Bebel-Str. 2, D-07743,

Jena,Germany,E-mail: c8wewo@uni-jena.de; (2) Institute of

Technical and Environment Chemistry, University of Jena, D-07743

Jena, Germany; (3) Max Planck Institute for Biogeochemistry,

D-07745 Jena, Germany; (4) Institute of Physical Chemistry,

Friedrich-Schiller-Universität August-Bebel-Str. 2, D-07743,

Jena, Germany

Ammonia is one of the most largely industrially produced basic compounds, leading to a variety of important secondary products. In the chemical industry, ammonia is produced in large amounts via the HABER-BOSCH-process. Compared with that, the nitrogenase enzyme operates in organisms under very mild conditions at atmospheric pressure and ambient temperature. Efforts are made to develop a method for the synthesis of ammonia from molecular nitrogen using $\mathrm{H}_{2} \mathrm{~S}$ and freshly precipitated iron sulfide thus serving as a primordial inorganic model for the enzyme nitrogenase. The reductant, as well as the reaction conditions (atmospheric nitrogen pressure and temperatures on the order of $70-80{ }^{\circ} \mathrm{C}^{1}$ are rather mild and therefore comparable to biological processes. The driving force of the overall reaction is believed to be the oxidation of iron sulfide to iron disulfide, and the formation of hydrogen from $\mathrm{H}_{2} \mathrm{~S}$. The reaction reported here supports the theory of an archaic nitrogen-fixing Fe-S cluster. Experiments with ${ }^{14} \mathrm{~N} /{ }^{15} \mathrm{~N}$ isotopes, Raman spectroscopic and X-ray powder diffractometry studies, respectively, are under investigation.

References

${ }^{1}$ W. Weigand, G. Kreisel, J. Käßbohrer, M. Sammler, R. Grunert, Procedure for the production of ammonia. Germany. DE 98-19846242(19846242), DE. 8-10-1998.

\section{UV Radiation, Water Content and Toxicology of the Martian Surface}

The Martian oxygen surface sink and its implications for the oxidant extinction depth

C. Kolb(1,2), H. Lammer(1), R. Abart(2), A. Ellery(3),

H. G. M. Edwards(4), C. Cockell(5), M. R. Patel(6)

(1) Department for Extraterrestrial Physics, Space Research Institute, Austrian Academy of Sciences Schmiedlstr. 6, A-8042 Graz, Austria, E-mail: christoph.kolb@oeaw.ac.at; (2) Institute for Mineralogy and Petrology, University of Graz, Universitätsplatz 2, A-8010 Graz, Austria; (3) School of Engineering, Kingston University, London, UK, E-mail: a.ellery@kingston.ac.uk; (4) Department of Chemical and Forensic Sciences, University of Bradford, West Yorkshire BD7 1DP, UK, E-mail: h.g.m.edwards@bradford.ac.uk; (5) British Antarctic Survey, BAS, High Cross, Madingley Road, Cambridge CB3 OET, UK, E-mail: csco@bas.ac.uk; (6) Planetary and Space Sciences Research Institute, The Open University, Walton Hall, Milton Keynes MK7 6 AA,UK,E-mail:m.r.patel@open.ac.uk
Based on the evolution of the atmosphere-surface-interaction of Mars, one might expect a large oxygen surface sink over geologic time-scales. Due to intense oxidation of inorganic matter this led to the formation of considerable amounts of sulfates and ferric oxides on Mars. To model this effect several factors have to be under consideration: Inorganic composition of the Martian soil, amount of incorporated oxygen, meteoritic gardening, and the oxidant extinction depth. The oxygen incorporation has further implications for the assumed oxidant extinction depth, which is an important parameter to determine required sampling depths on Mars to find putative organic material. 
The Martian atmospheric oxygen surface sink: a source for super-radicals

H. Lammer(1), H. Lichtenegger(2), C. Kolb(1,6), I. Ribas(3), E. F. Guinan(4), S. J. Bauer(5)

(1) Department for Extraterrestrial Physics, Space Research Institute, Austrian Academy of Sciences, Schmiedlstr. 6, A-8042 Graz, Austria, E-mail:helmut.lammer@oeaw.ac.at; (2) Space Research Institute, Department for Experimental Space Research, Austrian Academy of Sciences, Schmiedlstr. 6, A-8042 Graz, Austria, E-mail: herbert.lichtenegger@oeaw.ac.at; (3) Departament d'Astronomia $i$ Meteorologia, Universitat de Barcelona, Av. Diagonal 647, 08028 Barcelona, Spain, E-mail: iribas@am.ub.es; (4) Department of Astronomy and Astrophyisics, Villanova University, Villanova, PA 19085,USA,E-mail: guinan@ucis.vill.edu; (5) Institute for Geophysics, Astrophysics and Meteorology, University of Graz, Universitätsplatz 5, A-8010 Graz, Austria,E-mail:siegfried.bauer@ kfunigraz.ac.at; (6) Institute for Mineralogy and Petrology, University of Graz, Universitätsplatz 2, A-8010 Graz, Austria

Thermal loss processes of hydrogen and nonthermal atmospheric loss processes of hydrogen and oxygen, as well as chemical weathering of oxygen with the surface soil influence the evolution of the Martian atmosphere with regard to its water inventory. These oxygen atoms that react with the surface soil are responsible for the toxicology of the Martian surface. Since the evolution of thermal and nonthermal escape processes, such as exospheric loss of oxygen via dissociative recombination, atmospheric sputtering and ion pick up depend on the history of the intensity of the solar EUV radiation and the solar wind density, we use actual data from the Sun in Time program for reconstructing the Sun's history of the spectral evolution from X-ray to EUV from the observation of solar proxies with different ages from the present up to $3.5 \mathrm{Gyr}$ ago. Observations of flare activities of young solar-like stars inside the Sun in Time programme strongly suggest that flare events are frequent and more powerful than observed at the present Sun. The high X-ray activity and the fast rotation of the young solar-like stars indicate a much higher solar wind for the young Sun. We used a power law for the estimation of the average solar wind density of solar-like stars whose stellar winds were recently indirectly detected by using the amount of absorption in the $\mathrm{HI}$ areas as a diagnostic for their stellar mass loss rates. The correlation between mass loss and X-ray surface flux indicates a solar wind more than 1000 times massive in the distant past. We used a gas dynamic test particle model that involves the motion in the interplanetary electric and magnetic field for the estimation of pick up ion loss rates which seem to be the most efficient nonthermal loss process of the Martian atmosphere. By using new loss models and the data described above we estimate a loss of hydrogen and oxygen from Mars since $3.5 \mathrm{Gyr}$. In our evaluation of the water loss to space on Mars we found that all nonthermal escape processes of oxygen from present Mars cannot maintain the sum of thermal and nonthermal atmospheric loss rates of $\mathrm{H}$ in the ratio 2:1 at present. Escape to space could therefore not be the only sink for oxygen on Mars since the desirable ratio of 2:1 of $\mathrm{H}: \mathrm{O}$ loss rates should be established. Our study suggests that the missing oxygen needed for the validation of the $2: 1$ ratio between $\mathrm{H}$ and $\mathrm{O}$ is incorporated into the Martian surface by chemical weathering processes. Our results have important implications for exobiology and electromagnetic subsurface sounding techniques. The chemical environment, responsible for the oxidation of the Martian surface layers, is essentially also responsible for the toxicology of the Martian soil, since the reactivity is related to ionized radicals.

\section{Adsorption and capillary water in mid- and low latitude Martian soil}

\section{T. F. Möhlmann}

Institut für Raumsimulation, DLR, D-51170 Köln, Germany,

E-mail:dirk.moehlmann@dlr.de

It is shown that the water content of the soil of the upper Martian surface at mid and low latitudes is at least partially due to physisorbed 'adsorption water', which is in equilibrium with the atmospheric water vapor. This adsorption water covers the grains of the soil with multiple layers, and it does not freeze. It behaves like a fluid. This is the so-called 'unfrozen water in frozen soil' as it is observed in terrestrial permafrost at temperatures down to $-40{ }^{\circ} \mathrm{C}$. One monolayer of water molecules corresponds to about $37 \mathrm{~kg}$ water per cubic meter Martian soil. Thus, multiple layers are expected to host an amount of water of a few $100 \mathrm{~kg}$ water per cubic meter soil. This seems to be in agreement with Mars Odyssey results. As will be discussed, adsorption water is to be characterized by specific physical and chemical properties, if compared to bulk water or ice, and adsorption water can in case of sufficiently small pores or soil grains form capillary water.

\section{Sediment cycles on Mars in resonance with Earth}

R. R. Paepe, E. S. van Overloop

Geobound International Ltd and Free University of Brussels,

Doornstraat 27 B 9550 Herzele, Belgium,

E-mail: geobound@worldonline.be

Computation of cycles from sediment series on Earth has been linked up since Milankovitch to his well known astronomical cyclicities. Recurrent changes of Glacial and Interglacial Stages became mathematically bound to intricate aspects of the Solar System. The Milankovitch Cycles bound respectively to eccentricity, obliquity and precession of the Earth with relevant random periodicities of $100 \mathrm{~K}, 40 \mathrm{~K}$, and $20 \mathrm{~K}$-years could only give an explanation for the broader global climatic cold and warm changes during the Earth's Pleistocene Epoch. During the following Holocene Epoch covering in average the last $10 \mathrm{~K}$ years, shorter but nonetheless important global changes between wet and dry phases occurred as well. Geological sections revealed sediment series alternating with recurrent levels of palaeo-soil development. Geological correlation of these palaeo-soil sequences carried out by the authors on sediment sequences occurring all over the surface of the Earth then revealed the existence of twenty such levels at stratigraphically specific positions of the last 10000 years. The computation method ExSpect, an Expert Analysis Programme programme developed by Elfi Van Overloop, one of the present authors, revealed the existence of spectral peaks for the drought (soil) cycles interfering with the barren wet cycles of the sediment-deposits of the Holocene: 3183, 1853, 1163, 927, 513, 319, 298, 220, 146, 108 and 86 years. These spectra peaks compare perfectly with those obtained by NASA Goddard Space Flight Centre as of 893, and furthermore 514, 317, 298, 223, 149, 112 and 89 years for the Planetary Correlations. This was also clear indication that the Holocene Soil/ Climatic Changes responded to periodicities of the Solar System. Similar research carried out on sediment sequences found in images of Mars revealed not only the Milankovitch periodicities but shorter ones as well: 1000,4000 and the harmonic of the latter 9000 years. Smaller ones also appeared although their level of confidence is still under investigation. Nonetheless, these first results may point to a common origin of the Earth and Mars periodicities within the Solar System.

\section{Seasonal and diurnal variations of UV at the Martian surface}

M. R. Patel(1), J. C. Zarnecki(1), H. Lammer(2), C. Kolb(2,4), F. Selsis(3)

(1) Planetary and Space Sciences Research Institute, The Open University, Walton Hall, Milton Keynes MK7 6 AA, UK, E-mail:m.r.patel@open.ac.uk; (2) Space Research Institute, Department for Extraterrestrial Physics, Austrian Academy of Sciences, Schmiedlstr. 6, A-8042 Graz, Austria,

E-mail:christoph.kolb@oeaw.ac.at,helmut.lammer@oeaw.ac.at; (3) Centro de Astrobiologia (CSIC/INTA), Carretera Ajalvir, km 4, 28850 Torrejón de Ardoz, Madrid, Spain,E-mail:selsis@ observ.u-bordeaux.fr; (4) Institute for Mineralogy and Petrology, University of Graz, Universitätsplatz 2, A-8010 Graz, Austria

The solar UV radiation environment on planetary surfaces is of extreme importance in a wide range of scientific disciplines. Solar UV radiation is a driving force of organic and chemical evolution but serves also as a constraint in biological evolution. To gain an idea of how the martian UV radiation varies between different surface areas, including suggested landing sites of various future Mars landing probes, we modelled the total solar UV surface flux as a function of season and planetary latitude during one martian year, as well as daily UV irradiation conditions 
at martian perihelion and aphelion. A radiative transfer model has been developed, representing each gaseous/aerosol species present in the martian atmosphere, thus allowing modelling of any position on the martian surface at any time. The focus of this work is towards determining areas of possible future landing sites from an astrobiological perspective, taking into account the variation of UV irradiance across the Martian surface.

\section{North-south asymmetry of subsurface water distribution on Mars: implication from a global water cycle model \\ T. Tokano \\ Institut für Raumsimulation, DLR, D-51170 Köln, Germany, E-mail:tetsuya.tokano@dlr.de}

The Gamma-Ray Spectrometer onboard Mars Odyssey recently detected in the near-surface soil of the Martian polar region a high abundance of hydrogen, which is indicative of ground ice. In an effort to understand the observed global subsurface water distribution a global coupled atmosphere-subsurface water cycle model is developed. The simulation indicates that, regardless of the initial subsurface water distribution, the soil in the northern hemisphere becomes more wet compared to the southern hemisphere under the present climate. This hemispheric asymmetry is caused by a combination of a higher ground ice sublimation rate in the south, stronger northward transport of atmospheric water, and the presence of the northern residual polar cap as a major atmospheric water source. The presence of ground ice at the very top of the soil is unlikely because otherwise it would cause an excessive abundance of atmospheric water. The latitudinal water distribution in the top $60 \mathrm{~cm}$ of the soil inferred by Mars Odyssey generally seems to be consistent with the present global water cycle, while the origin of deeper water remains ambiguous.
Identification of shallow subsurface water on Mars and its relevance for the selection of the ExoMars mission's landing site

J. L. Vago and the ExoMars Study Team

European Space Agency, ESA/ESTEC, Keplerlaan 1,

NL-2201 AZ Noordwijk, The Netherlands, E-mail:

jorge.vago@esa.int

Without water, the metabolic activities of living cells would not be possible. In the absence of water, life either ceases or slips into a quiescent mode. Hence, a search for extant life on Mars automatically translates into a search for an environment capable of sustaining - at least episodically - liquid water. Yet, water is presently not stable on the Martian surface: its temperature and atmospheric pressure are too low. Still, water/ice may lie at some depth underground, or exist in highly localized areas where ambient conditions may be more favourable. In this regard, an interesting development has been the recent measurements performed by Odyssey's gamma-ray spectrometer. ${ }^{1}$ They have revealed, for latitudes greater than $40^{\circ}$, the presence of extensive ice deposits in the 1-m topsoil layer. This information, combined with topographic, temperature, and pressure data from previous missions/model results, ${ }^{2}$ allows us to begin the necessary discussions to identify possible landing sites of interest for ESA's ExoMars mission. This paper also presents those engineering requirements that may constrain the selection of landing sites.

References

${ }^{1}$ Distribution of hydrogen in the near surface of Mars: Evidence for Subsurface Ice Deposits, W. V. Boynton et al., Science 297, 81-84 (2002).

${ }^{2}$ Use of Spacecraft Data to Derive Regions on Mars Where Liquid Water Would Be Stable, B. Lobitz et al., PNAS 98, 2132-2137 (2001).

\section{Potential Martian Habitats - Earth Analogues}

\section{Ultraviolet protection in micro-habitats - lessons from the terrestrial poles applied to Mars \\ C. S. Cockell(1), P. Rettberg(2), G. Horneck(2), M. Patel(3), \\ H. Lammer (4), C. Cordoboa-Jabonero(5) \\ (1) British Antarctic Survey, BAS, High Cross, Madingley Road, Cambridge.CB13AR,UK,E-mail: csco@bas.ac.uk; (2) Institute of Aerospace Medicine, Radiation Biology Section, DLR, Linder Höhe, D-51147 Cologne, Germany, E-mail: gerda.horneck@dlr.de; (3) Planetary and Space Sciences Research Institute, The Open University, Walton Hall, Milton Keynes MK7 6 AA, UK, E-mail: m.r.patel@open.ac.uk; (4) Department for Extraterrestrial Physics, Space Research Institute, Austrian Academy of Sciences, Schmiedlstr. 6 A-8042 Graz, Austria, E-mail:helmut.lammer@oeaw.ac.at; \\ (5) Centro de Astrobiologia (CSIC-INTA), Carretera Ajalvir, km 4, 28850 Torrejón de Ardoz, Madrid, Spain}

The Martian UV flux is approximately three orders of magnitude more detrimental to DNA than on Earth and yet there are a variety of microhabitats under which effective protection from UV radiation can be achieved. Using the DLR-Biofilm dosimeter, we have quantified protection afforded by soils, snow and ice and rocks in an artic polar desert. We demonstrate that in the endolithic habitat at a depth of $\sim 1 \mathrm{~mm}$ spore inactivation is reduced by two orders of magnitude, but enough light can penetrate for photosynthesis to occur. Under soil layers of $\sim 0.5 \mathrm{~mm}$, spore inactivation in unmeasurable even after $5 \mathrm{~d}$ of exposure. Snow and ice of thickness 5 to $10 \mathrm{~cm}$, depending on age and heterogeneity, can reduce spore inactivation and DNA weighted irradiance by an order of magnitude, such that the effects of ozone depletion in the Antarctic during the spring can be completely counteracted by thin snow and ice covers. We directly quantified the attenuation properties of snows at $254 \mathrm{~nm}$ under field conditions, showing that dense Martian north polar ice (approximately three times denser than Antarctic snow) could reduce UV flux by an order of magnitude at $\sim 5 \mathrm{~cm}$ depth or less. These data provide insights into the way in which substrates available in polar deserts can protect microorganisms from UV radiation and how effective they might be on Mars.

\section{Photosynthetic microorganisms and their remnants in polar environment - possible markers after a long term change \\ J. Elster \\ Institute of Botany, Academy of Sciences of the Czech Republic, CZ 37982 Trebon, Czech Republic, E-mail: jelster@butbn.cas.cz} Cyanobacteria and algae marked the beginning of plant evolution. Thanks to their evolutionary antiquity they are widely adapted to all extremes associated with changes that have occurred in geological time. They possess an oxyphototrophic type of photosynthesis. Cyanobacteria and algae are common components of most of the present Earth's ecosystems. The importance of their role as primary producers of organic matter increases with the extremity of environment. The polar terrestrial environment is extreme in temperature, light (including UV radiation), desiccation and freeze-thaw cycles, and in changes in mineral salt concentration. The presence of liquid water acts as a buffer of temperature and dissolved solids and nutrients changes by having a large heat storage and dilution capacity, and generally stabilizes the environment near the ground surface. In the polar terrestrial environment the pattern of cyanobacteria and algae distribution is associated with the liquid water availability gradient; aquatic (planktonic communities) semi-aquatic (hydro-terrestrial communities) and dry (terrestrial communities). After long-term climatic changes from cyanobacterial and algal biomass and later on from its various remnants in pristine ('Martian') ecosystem we can learn a lot of about environmental history (water availability) and evolution of studied habitats. For the last few years, I have been working in ecology and ecophysiology of cyanobacteria and algae in polar hydro-terrestrial and terrestrial ecosystems. 
In my presentation I will introduce some information from my previous studies. I believe that my experience could bring potential interdisciplinary cooperation with colleagues working in various subjects of Exo/Astrobiology.

\section{Methanotrophic bacteria in movile cave,} a chemoautotrophically based ecosystem

\section{E. Hutchens(1), S. Radajewski(2), C. Murrell(2)}

(1) Mineralogy Department, The Natural History Museum, Cromwell Road,London,SW7 5BD, UK, E-mail: elenahutchens@yahoo.com; lilh@nhm.ac.uk; (2) Department of Biological Sciences, University of Warwick, Coventry CV4, 7AL, UK

Movile Cave is a ground-water ecosystem in southern Romania that is supported by in situ autotrophic production, and contains the only terrestrial community known to be chemoautotrophically based. As the methane concentration in the atmosphere of the cave is approximately $1 \%(\mathrm{v} / \mathrm{v})$, we attempted to isolate and characterize the methanotrophic bacteria from this unusual environment. We have used three approaches to characterize the methanotroph population. The first, indirect, approach attempted to enrich, isolate and characterize the methanotrophic bacteria. The second approach relied on polymerase chain reaction (PCR) technology and the use of functional gene (particulate methane monooxygenase - pmoA) primers for detection and analysis of methanotrophs directly from environmental samples. The results of these studies indicated that the population of methanotrophic bacteria in Movile Cave consists of both type I (assimilate formaldehyde produced from the oxidation of methane using ribulose monophosphate pathway) and type II methanotrophs (utilize the serine pathway for formaldehyde assimilation). The third approach used the recently developed technique of stable isotope probing to identify the active methanotrophs in a sample of cave mat and cave water. When incubated in the presence of $13 \mathrm{CH}_{4}(99 \% 13 \mathrm{C})$ in flasks, the DNA of metabolically active methanotrophs becomes 13C-DNA as a consequence of growth on the isotopically labelled substrate. The 'heavy' 13C-DNA fraction was resolved from the 'lighter' 12C-DNA by density-gradient centrifugation, purified and used as a template for PCR. Specific products were obtained with primer sets that amplify eubacterial and eukaryal small-subunit rRNA genes and the functional gene pmoA. DNA sequencing of clones from each of the PCR products indicated that type I methanotrophs form the predominant group of active methanotrophs in Movile Cave.

\section{Possible photosynthetic microbes in the dark dune spots}

T. Gánti(1), A. Horváth(2), E. Szathmáry(1)

(1) Collegium Budapest, Institute for Advanced Studies,

2 Szentháromság utca, H-1014, Budapest, Hungary, E-mail:

szathmary@colbud.hu; (2) Budapest Planetarium,P.O.B.47,

H-1476 Budapest, Hungary,E-mail:planet@mail.datanet.hu

Dark dune spots (DDSs) occur as ephemeral objects on the dark dune (DD) fields, which are fine-grained, dark blue, low-albedo, sand-sized eolian sediments, mainly of dense basaltic sand. DDSs begin to appear during late winter and spring on the ice cover of intracrater dark sand sheets, in the southern polar region. DDS dynamics has initially been interpreted as resulting from $\mathrm{CO}_{2}$ and $\mathrm{H}_{2} \mathrm{O}$ sublimation and re-precipitation, although this explanation is clearly incompatible with many of the observed traits. The bulk radial symmetry, the flowing (seepage) patterns and the defrosting beginning from bottom of DDSs are strongly suggestive of some kind of biological activity. Under Martian circumstances the only possible solvent is liquid water (with some salt component). We have interpreted the sequence of DDS formation and the seasonal and annual changes (documented by MOC images) as a biomarker. If Martian Surface Organisms (MSOs) exist, they could dwell below the surface ice, which is heated up by their absorption of sunlight. Later they grow and are reproduced through photosynthesis, thus contributing to their own living conditions. Some terrestrial analogues seem to support this hypothetical alien lifecycle.

References

Horváth A., Gánti T., Gesztesi A., Bérczi Sz., Szathmáry E. (2001a) LPS 32.
Gánti T. et al. (2002a) LPS 33.

Horváth A. et al. (2002b) LPS 33.

Horváth A. et al. (2002c) LPS 33.

Horváth A., Gánti T., Bérczi Sz., Gesztesi A., Szathmáry E. (2002d)

Antarctic Meteorites 2, pp. 37-39.

\section{Was Earth ever infected by Martian biota? Clues from radioresistant bacteria \\ A. K. Pavlov(1), V. L. Kalinin(2), A. N. Konstantinov(2),}

V. N. Shelegedin $(2)$

(1) Ioffe Physico-Technical Institute, Russian Academy of Sciences, Russia; (2) also at St Petersburg Technical State University, Russia We propose a new scenario for the Martian origin of radioresistant microorganisms such as Deinococus radiodurance, Rubrobacter radiotolerance, Rubrobacter xylanophilus. It is well known that high tolerance to radiation is not necessary for surviving in Earth environment. The radiation background on Earth is many orders of magnitude lower than the lethal dose for these microorganisms. Our hypothesis is based on the experiments demonstrating the ability of ordinary bacteria, such as Escherichia coli, to develop radioresistance after a series of cycles of high dose irradiation (with doses up to the almost lethal one) and following recovery of population. We show that processes of this kind could take place in natural environments only on Mars. Our speculations are based on the well-known astronomical theory of Martian climate oscillation and our calculations of radiation levels in the surface layer of the planet. According to our calculations hypothetical Martian microorganisms could develop radioresistance in just several millions years. From the study of Martian meteorites we assume that a transfer of Martian radioresistant microorganisms to Earth could occur more than once. As a result, several different kinds of radioresistant microorganisms could exist on Earth. We discuss the special features of Deinococus radiodurance that indicate a possible extraterrestrial origin. In the conclusion we propose that Mars polar regions (ice and regolith, upper tens $\mathrm{cm}$ - several meters under the surface) are the most promising for exploration of possible Martian life by space missions.

\section{Microbial endolithic biofilms as a means of survival in the harsh antarctic conditions}

A. de los Ríos(1), J. Wierzchos(2), L. G. Sancho(3), M. Grube(4), C. Ascaso(1)

(1) Centro de Ciencias Medioambientales (CSIC), Serrano 115 dpdo. Madrid-28006 Spain,E-mail: arios@ccma.csic.es; (2) Servei de Microscopia Electronica, Universitat de Lleida, Rovira Roure 44. Lleida-25198. Spain; (3) Dpt. Biolog Vegetal II, Universidad Complutense de Madrid, 28040, Madrid, Spain; (4) Institut für Botanik, Karl Franzens Universität Graz, Holteigasse 6, 8010 Graz, Austria

Antarctic ecosystems are one of the most interesting extreme environments available for microbial diversity and ecological studies related to the astrobiology. Much of biota of this continent is restricted to endolithic microecosystems, which harbor distinct microbial communities constituted in biofilms. These films can be viewed as systems immobilized in the rock and embedded in an organic matrix resulting from the excretion of exopolysaccharides by resident microorganisms. The lithic substrate and the microorganisms comprising these films are intimately linked, and this gives rise to complex mineral-microbe interactions. Hence, the microhabitats and microenvironments of endolithic microecosystems are not only determined by the physicochemical features of the lithic substrate, but are also conditioned by the biological components of these biofilms. The Antarctic biofilms analysed in this study are characterized by the presence of extracellular polymer substances and of acid microenvironments in the proximity of the cells. Cyanobacteria appeared as key components of these biofilms in zones where there were no lichen thalli nearby. Fungal cells were the predominant organisms in zones where epilithic lichens were present. The combination of microscopical and molecular techniques have permitted recognition of different biological components present within the lithic substrate. Biofilm structure in Antarctic ecosystems may favor the 
formation of microsites with specific physicochemical conditions, which permit survival of microbial communities in this extreme environment.

\section{Extremophiles in ice and snow of Antarctica and the Alps \\ B. Sattler \\ Institute of Zoology and Limnology, University of Innsbruck, Technikerstrasse 25, A-6020 Innsbruck, Austria, E-mail: birgit.sattler@uibk.ac.at}

Until recently, the vast snow and ice fields of remote cold areas like the Alps or polar regions have always been seen as sterile landscapes.
However, with the understanding that microbes can remain in ice at temperatures well below zero and can be revitalized when brought above freezing point, these areas can be recognized as a huge ecosystem. With the requirement of periods allowing a liquid microfilm of water formed around the cell metabolism and degradation of various substances can take place. Ice ecosystems are not solely present on the surface of our planet but also in the atmosphere where we detected viable bacteria with active metabolism meaning that cells are not just distributed by air parcels but are also actively growing at the most extreme conditions (low temperature, low pressure, high UV, low nutrients). Results are presented herewith from the Austrian Alps, Antarctica and atmospheric studies, representing the variety of extremity in ice ecosystems.

\section{Astrobiological Relevant Instrumentation for Solar System Exploration}

\author{
Survey of instruments for planetary exploration \\ P. Falciani, G. Preti \\ Galileo Avionica SpA, Via Albert Einstein, 35, 50013 Campi Bisenzio \\ (FI), Italy, E-mail: piergiuseppe.falciani@officine-galileo. \\ finmeccanica.it
}

Several missions for planetary explorations, including comets and asteroids, are planned by the Space Agencies: BepiColombo, Rosetta, Mars Sample Return etc. This fact gives rise to the necessity of several kinds of instrumentation/facilities for planet surface observation and investigation, both by orbiter or in situ. The aim of this paper is to give an overview of some of these instruments and in particular those devoted to work on the surface of the planet under observation. These instruments not only measure the characteristics of the soil, e.g. temperature, humidity, magnetic field, composition, but are also devoted to the search for life. The article covers the Galileo Avionica activities related to the studies already done or ongoing, under ESA and ASI contracts, like the Multi-user Facility for Exobiology Research or DEEDRI and IPSE.

\section{Remote sensing of circularly polarized light from orbit of planet Mercury by the ESA Mission BepiColombo}

U.J. Meierhenrich(1), W. H.-P. Thiemann(1), B. Barbier(2), A. Brack(2), C. Alcaraz(3), L. Nahon(3), Ray Wolstencroft(4)

(1) Department Physical Chemistry, Bremen University, FB 02, NW 2, B 1056 Leobener Straße, D-28359 Bremen, Germany, E-mail: mhenrich@uni-bremen.de; (2) Centre de Biophysique Moléculaire, CNRS, Rue Charles Sadron, F-45071 Orl Cedex, France; (3) LURE, bât 209d, Universität Paris-Sud, BP 34, F-91898 Orsay Cedex, France; (4) Royal Observatory Edinburgh, Blackford Hill, Edinburgh

\section{EH9 3 HJ, Scotland, UK}

Circularly polarized electromagnetic radiation can be measured with earth based telescopes in galaxies, stars, the interstellar medium and planets. The observed values for Venus, the Moon, Mars, and Jupiter obtained by photo-polarimetric measurements were 'symmetric' and showed accordance with theory. However, for planet Mercury asymmetric parameters in the circular polarization were measured that do not fit with calculations. For BepiColombo, the ESA cornerstone mission 5 to Mercury, we propose to investigate this phenomenon using a concept which includes two instruments. ${ }^{1}$ The first instrument is a highresolution optical polarimeter, capable of determining and mapping the circular polarization by remote scanning of Mercury's surface from the Mercury Planetary Orbiter MPO. The second instrument is an in situ sensor for the detection of the enantiomorphism of surface crystals and minerals, proposed to be included in the Mercury Lander MSE. With this combination of instruments we hope to supply crucial information on the origin of biomolecular asymmetry. The required instrumentation will be presented and discussed.

\section{References}

1 U. J. Meierhenrich, W. H.-P. Thiemann, B. Barbier, A. Brack, L. Nahon, C. Alcaraz, R. Wolstencroft: Circular Polarization of Light by Planet Mercury and Enantiomorphism of its surface minerals. Origins of Life and Evolution of the Biosphere 32 (2002), in print.

\author{
Interpretation of data recovered from space \\ missions: decoding of complex chromatograms \\ by Fourier analysis \\ M. C. Pietrogrande \\ Department of Chemistry, University of Ferrara, Via L. Borsari, \\ 46 I44100 Ferrara, Italy, E-mail:mpc@unife.it
}

One of the main objectives of space probes is to characterize the chemical composition of extraterrestrial environments., i.e., Huygens and Rosetta missions are devoted to investigation of Titan and comet $\mathrm{P} /$ Wirtanen atmospheres due to their relevance to planetology and exo/astrobiology. In the scientific payload of the probes, gas chromatography (GC) plays a predominant role to separate and identify the species present in these complex environments. ${ }^{1,2}$ In spite of development of proper instrumentations for space application, limitations and instrumental constraints imposed by the space environment prevent us from obtaining the optimum separation of the analysed species. Therefore, complex chromatograms are obtained from in-situ space analysis, and a mathematical approach is required to decode the involved signals and interpret data recovered from the space instruments.

In this paper a chemometric approach based on the Fourier analysis is reported. It is based on the computation of Autocovariance Function (ACVF) on the experimental chromatogram. ${ }^{3}$ ACVF plot constitutes a fingerprint of the mixture since, in contrast with the original chromatogram very crowded with peaks, it retains in a simpler plot all the information on the nature and relative abundance of the compounds present in the mixture. From the ACVF plot, information on the atmospheric complexity as well as on the separation system can be easily extracted: the number of components present in the sample can be estimated, the performance of the analytical separation can be evaluated, the presence of specific molecular species can be singled out by the identification of an ordered retention pattern. ${ }^{4,5}$ Applications are described specifically developed in sight of interpreting the complex chromatograms to be collected in space missions: complex samples, simulating extra-terrestrial atmosphere compositions, where analysed in the same GC analysis conditions (temperature, outlet pressure) operated by the Rosetta Lander probe. The method is helpful when interpreting the data recovered from space missions, since it allows the chemical characterization of the extraterrestrial matter, to calibrate space instruments and to determine the best operating conditions for these instruments compatible with space constraints.

References

1 R. Sternberg, C. Szopa, D. Coscia, S. Zubrzycki, F. Raulin, C. Vidal-Madjar, H. Niemann, G. Israel, J. of Chromatogr. A. (1999), 846, 307-315.

2 C. Szopa, R. Sternberg, D. Coscia, H. Cottin, F. Raulin, F. Goesmann, H. Rosenbauer, J. of Chromatogr. A. (1999), 863, 157-169.

3 A. Felinger, L. Pasti and F. Dondi, Anal. Chem. (1991), 63, 2627-2633.

4 A. Felinger, M. C. Pietrogrande, Anal. Chem. (2001), 73, 618A-626A.

5 M. C. Pietrogrande, P. Coll, R. Sternberg, C. Szopa, R. NavarroGonzalez, C. Vidal-Majar, F. Dondi, J. of Chromatogr. A. (2001), 939, 69-77. 


\section{Astrobiological relevance and feasibility of a sample collection mission to the atmosphere of Venus} D. Schulze-Makuch(1), L. N. Irwin(2), T. Irwin(2)

(1) Department of Geological Sciences, University of Texas, El Paso 500 W, TX 79968-0555 USA, E-mail:dirksm@geo.utep.edu; (2) Department of Biological Sciences, University of Texas, El Paso 3 Texastronautics San Pedro, California, USA

The lower cloud level of the Venusian atmosphere is an environmental niche that could harbor life. We base this statement on the observations that (1) the lower cloud layer contains non-spherical particles with an average diameter of 3.6 micrometers comparable in size to Earth microbes, (2) conditions at this cloud level are relatively benign at a temperature of $300-350 \mathrm{~K}$, about 1 bar pressure and a $\mathrm{pH}$ of 0 , (3) the superrotation of the atmosphere in 4 to 6 days around the planet shortens the duration of the night, hence enhancing the potential for photosynthetic reactions, (4) a UV adsorber has been detected in the atmosphere, which may be related to the larger cloud particles, (5) while water is scarce on Venus, water vapor concentrations in the lower cloud layer reach several hundred ppm. Microbes, if they exist in this layer, may be able to assimilate water vapor from the atmosphere similar to microbes on Earth that can assimilate $\mathrm{CO}_{2}$ from Earth's atmosphere, and (6) there is evidence that an ocean existed on Venus in its early history. If so, microbial life could have originated under surface water conditions, and then retreated to the environmental niche of the lower cloud layer by adapting progressively through directional selection as surface conditions on Venus became untenable. Or, alternatively, microbes could have been delivered by meteorites from Earth or Mars. Many of the early Earth microbes were thermoacidophilic, which would have made adaptation to Venusian conditions easier. We have previously argued on the basis of the reasoning above that a sample return mission from the lower cloud layer of the Venusian atmosphere should be considered. Here we identify methods for missions that include a sample return or in-situ analysis, and analyse their merits. The first option considered is a Stardust-like probe with Aerogel panels to collect the micrometer cloud particles and analyse them in-situ or return them to Earth. The second option is an atmospheric probe deployed and retrieved from an orbiting mother craft by tether to collect a sample at the altitude of interest. The third option involves a sample collector that would be released to descend by parachute through the atmosphere and analyse cloud particles in situ.

\section{Astrobiotechnology for solar system exploration}

A. Steele(1), J. Maule(1), J. Toporski(1), M. Schweitzer(2),

R. Avci(2), H. Rowlands, S. Pincus, V. Parro-Garcia(3),

C. Briones(3), R. Robertson(4), D. S. McKay(5)

(1) Carnegie Institution of Washington 5251 Broad Branch Rd., Washington DC, USA, E-mail: a.steele@gl.ciw.edu; (2) Montana State University, Bozeman Montana, USA; (3) Centro de Astrobiologia (INTA-CSIC), Carretera de Ajalvir km 4, Torrejón de Ardoz. Madrid, Spain; (4) Oceaneering Space Systems, Space Center Blvd. Houston Texas, USA; (5) Astrobiology Group, Johnson Space Center, Houston 77058 Texas, USA

With the advent of a new era of Astrobiology missions to search our solar system for evidence of life we would like to again present a new approach to this goal. We have reviewed the current list of biotechnology techniques, which are applicable to miniaturization and integration into a combined flight platform. Amongst the techniques reviewed are; the uses of antibodies, fluorescent detection strategies protein and DNA chip technology, surface plasmon resonance and its relation to other techniques, micro electronic machining (MEMS where applicable to biological systems), nanotechnology (where applicable to biological systems), lab on a chip technology (including PCR), mass spectrometry (i.e. MALDI-TOF) fluid handling and extraction technologies. Chemical Force Microscopy (CFM). Raman Spectroscopy nanotechnology (i.e. molecular motors). We have begun to integrate this knowledge into a single flight instrument approach for the sole purpose of combining several mutually confirming tests for life, organic/ microbial contamination, prebiotic and abiotic organic chemicals. We will present several innovative designs for new instrumentation based on protein and DNA chip technology including pro-engineering design drawings of a protein chip reader for space flight and fluid handling strategies. We will also review the use of suitable extraction methodologies for use on different solar system bodies.

\section{On the detection of bacterial biomarkers and the implications for astrobiology}

J. Toporski(1), A. Steele(1), F. Westall(2), R. Avci(3), D. S. McKay(4)

(1) Geophysical Laboratory, Carnegie Institution of Washington,

5251 Broad Branch Road NW, Washington DC, USA 20015-1305,

USA, E-mail: j.toporski@gl.ciw.edu; (2) Centre de Biophysique

MoléculaireCNRS, Rue Charles Sadron, 45071 Orléans, France,

(3) Montana State University, ICAL, Bozeman, MT, USA; (4) NASA Johnson Space Center, Mail Code SN, Houston TX 77058, USA

Unequivocal detection of bacterial biomarkers is an important challenge in our venture to determine strategies how to best recognize signatures of life rocks, one of the stated main goals of the NASA Astrobiology Road Map, the need to be able to recognize the signature of life in rocks from Earth's fossil record as well as in ex-traterrestrial materials. As has become apparent in the current debate over the true nature of the earliest evidence of life on Earth, ${ }^{1,2}$ we still have difficulties in unambiguously identifing this possible evidence. In the astrobiology community, this issue has attracted intense discussion for over five years, regarding the possible evidence of past biogenic activities in Martian meteorite ALH84001, ${ }^{3,4}$ with yet no conclusive answer on hand. To complicate matters, the issue of distinguishing between evidence of past life and evidence of viable life in extraterrestrial materials became apparent through the discovery of recent microbial contaminants in ALH84001, although this meteorite was considered to contain no evidence of life by others. As a valuable offspring of these discussions on benchmark research it emerged that it is possible to tackle this challenge by multidisciplinary and thus multitechnical means. We will present a number of multidisciplinary and multi-technical approaches we successfully applied in our studies on fossil bacterial biofilms and the search for evidence of life in meteorites, including techniques such as Time of Flight Secondary Ion Mass Spectroscopy (ToF-SIMS) in combination with electron microscopy and X-ray analytical tools, as well as the application of biotechnology.

References

${ }^{1}$ Schopf et al. Nature (2002), 416, 72-76.

${ }^{2}$ Brasier et al. Nature (2002), 416, 76-81.

${ }^{3}$ e.g. McKay et al. Science (1996), 273, 924-930.

${ }^{4}$ Treiman. 32nd LPSC, Abstr (2001), 1304.

${ }^{5}$ Steele et al. MAPS (2000), 35, 237-241.

\section{Venus as an exobiological object}

M. Yamauchi(1), J.-E. Wahlund(2), S. Barabash(1)

(1) Swedish Institute of Space Physics (IRF), Box 812 S-981 28, Kiruna, Sweden,E-mail: stas@irf.se; (2) Swedish Institute of Space Physics, Uppsala Division, Box 537 SE-751 21, Uppsala, Sweden, E-mail:jwe@irfu.se

Venus is one of the exobiological objects in the sense that it could have been the first planet where ancient prebiotic molecules were formed in the solar system, that its atmospheric environment provides the most possible aerosol condition is important for the origin of life in the solar system, and that even if there might be no life it provides good references for the chemical reactions in the similar way as the biosphere does. One forgotten argument in the past Venus exobiology studies is the speciality of its polar region. It might be cool if the atmospheric super-rotation dies there. Also the cloud in the center of the polar vortex can survive for a quite long time that biological processes could take place. Thus, even an atmospheric mission to Venus may contribute to Venus exobiology if it carefully covers the polar region. We discuss currently proposed Venus missions from the exobiological viewpoint. 


\section{Subsurface and Atmospheres of Icy Worlds}

Melting probe at Lake Vostok and Europa

J. Biele(1), S. Ulamec(1), S. J. Barber(2), I. P. Wright(2)

(1) Deutsches Zentrum für Luft- und Raumfahrt, Institut für

Raumsimulation, DLR, Linder Höhe D-51147 Köln, Germany, E-mail: jens.biele@dlr.de; (2) Open University, Milton Keynes MK76AA,UK The Jovian satellite Europa has the potential to offer yet further insights into those environments, which may be conducive to life. A wide range of exploration techniques exists, from fly-bys, to orbiters, to landers. One technology that will be needed will be some sort of device that is capable of melting its way through the satellite's outer ice crust, and down towards the putative ocean of liquid water. In order to understand the physical and chemical nature of the ice layers that constitute the Europan crust, as well as hopefully being able to sample any underlying ocean water, any probe should include a suite of scientific instruments, including ones that are capable of determining the chemical and isotopic compositions of the constituent Europan materials. In this regard there is experience in the team in the development of light-weight gas chromatograph - mass spectrometer systems, as provided for the Rosetta lander ${ }^{1,2}$ and a small mass spectrometer $(<500 \mathrm{~g})$ that could fit inside the burrowing device proposed for the surface element of the BepiColombo mission to Mercury. A demonstration mission can be foreseen at Antarctica with a very simple probe, that melts through the ice, and takes a sample of e.g. Lake Vostok, which is an excellent analogue of Europan ice crust/ocean. Since the energy demand is high, in case of extraterrestrial application (e.g. Europa), only heating with radioactive material seems feasible. The necessary power is driven by the desired penetration velocity (almost linearly) and the dimensions of the probe (third power). The choice of the radioactive heater isotope needs to be in accordance with environmental regulations. Sample collection and retrieval could be achieved by means of a pressure valve and the release of a weight, so that the probe has buoyancy and takes the sample back to the surface. The sample, once collected at the surface, could subsequently be analysed by a full suite of analytical techniques including spectroscopic and microbiological analyses. In case of Europa such a probe needs to be larger to allow in-situ analysis. Data transmission to the surface element would be performed with long wave technology.

\section{Introduction to the Permafrost Astrobiology programme \\ D. A. Gilichinsky}

Institute of Physicochemical and Biological Problems in Soil Science, Russian Academy of Sciences, Soil Cryology Laboratory, 142290,

Puschino, Institutskaya Str. 2, Moscow region, Russia, E-mail: gilichin@issp.serpukhov.s

The search strategy for Extraterrestrial life must be based on studies of the most probable environments on Earth where Life might be found. Seven from the nine planets of the solar system and their moons, and comets/asteroids are of a cryogenic nature, i.e. the Cryosphere is a common phenomenon in our Cosmos. The Terrestrial Cryosphere (ice sheets, glaciers, subsurface ice veins or intrusions, overcooled salt water and rooks, permafrost soils) is a depository of ancient biosignatures analogues of possible Extraterrestrial ecosystems: biogases, biominerals, pigments, lipids, enzymes, proteins, nucleotides, RNA/DNA fragments \& molecules, dead and viable cells. The microorganisms, their traces or metabolic end products in the Earth Cryosphere represent a significant part of the Biosphere, the Cryobiosphere, whose most inhabited part is Permafrost. The subzero temperatures make it one of the more stable natural environments. This environment provides life support and ensures the formation of microbial communities that realize unknown possibilities of physiological and biochemical adaptation to prolonged cold and remain virtually stable for millions of years. Permafrost is characterized by a specific, multiphase state of water, which plays a dual role from the biological point of view. The solid phase (ice) makes up most of the total water volume in permafrost and serve as a conservator for biological objects. The remaining part of the water is in an unfrozen state, most commonly as thin films that serve as protector and play a leading role in the cell preservation. Unfrozen water might be considered as a main ecological niche where the microorganisms might survive. Unfrozen water represents firmly bound liquid water with binding molecules, and may exist, for example, in Martian permafrost. Viable organisms found in the Earth permafrost provide a range of potential inhabitants on the Space cryogenic bodies, i.e. Extraterrestrial Cryospheres represent environments where the probability of finding life is extremely high. If life existed on earth-like planets during the early stages of evolution, then its traces at the cell level might have been preserved at permafrost depths that probably contain the genetic resources of life, vanished due to the catastrophic events on the planets. This is why Permafrost Astrobiology should be an inherent part of the Extraterrestrial Life Programme.

\section{The Cassini/Huygens mission to Saturn and Titan}

J.-P. Lebreton(1), B. Kazeminejad(1), D. L. Matson(2), L. Spilker(2) (1) Research and Scientific Support Department of ESA, ESTEC, P.O. Box 299, 2200 AG Noordwijk, The Netherlands, E-mail: jeanpierre.lebreton@esa.int; (2) Jet Propulsion Laboratory, California Institute of Technology, 4800 Oak Grove Drive, Pasadena, CA 91109, USA

The Cassini/Huygens spacecraft was launched in October 1997. Since that time it has been on an interplanetary trajectory toward Saturn. The spacecraft comprises a Saturn orbiter and a Titan entry probe. It arrives at Saturn and goes into orbit about the planet on 1 July 2004. Cassini will perform seventy-five orbits around Saturn during the nominal four-year mission. Among them, forty-four include close flybys of Titan. Huygens is an entry probe designed to descend by parachute through the atmosphere, down to the surface, of Titan, Saturn's largest moon. The Probe mission is now planned for the third orbit, on 14 January 2005, after two fly-bys of Titan (in October and December 2004). Huygens will be released about three weeks before it reaches Titan. This paper provides an overview of the mission with emphasis on Titan's exploration. Part of this work was carried out at the Jet Propulsion Laboratory, California Institute of Technology, under contract to NASA.

\section{Titan's surface: what will we find? \\ R. D. Lorenz \\ Lunar Planetary Laboratory (LPL), University of Arizona, \\ 1629 E. University Blvd, Tucson, AZ 85721-0092, USA, \\ E-mail: rlorenz@lpl.arizona.edu}

This talk will summarize the present understanding of Titan's surface environment: clues from ground-based radar and infrared studies point to a varied surface, in sharp contrast to the bland appearance of the organic haze. The most likely scenario is of organic deposits - including large seas of liquid ethane - over, and occasionally mixed with, ice bedrock. Physical arguments can constrain the likely vigour and nature of processes, such as wind-driven waves and tides in the lakes, erosion by methane rainfall, and the relative energies of tidal dissipation and radiogenic heat from the interior which may drive cryovolcanism and ice tectonics over an internal water-ammonia ocean. Impact cratering is likely to be a ubiquitous process (albeit different due to the thicker atmosphere than on other icy satellites) and a useful probe of the interior. Impact melt and cryovolcanism offer the prospect of aqueous interaction with nitriles to yield more complex compounds of exobiological interest. Finally, as Cassini planning reaches an advanced stage, an impression of the Titan that will be unveiled to us two years from now can be drawn, and ideas for future missions formulated. 
Titan chemistry and exobiology: new laboratory data and implications for Cassini-Huygens

F. Raulin(1), Y. Bénilan(1), J.-M. Bernard(1), P. Coll(1), A. Jolly(1), M.-C. Gazeau(1), F. Shindo(1), V. Vuitton(1), J.-C. Guillemin(2) (1) LISA, UMR CNRS 7583, Universites Paris 12 and Paris 7 , 61 Avenue du Général de Gaulle, F-94010 Creteil, Cedex, France, E-mail:raulin@lisa.univ-paris12.fr; (2) Laboratoire de Syntheèses Activations de Biomol, CNRS, ENSCR, 35700 Rennes, France Since the discovery of a dense N2-CH4 atmosphere on Titan, the largest satellite of Saturn, several organic compounds have been detected - as expected - in this environment and many others are supposed to be present. Complementary approaches have been systematically followed to study the organic chemistry that is going on in this planet-sized laboratory: experimental simulation in the laboratory, using methanenitrogen mixtures models of Titan's atmosphere theoretical modelling, with the development, in particular, of 0-D to 3-D photochemical models direct observation of Titan's atmosphere by remote sensing techniques (before the availability of in-situ analysis in the frame of the Cassini-Huygens mission). Several new results have been obtained recently concerning different exobiological aspects of Titan: discovery of water (in gas phase and at the ppb level) by remote sensing techniques; observation of Titan's surface showing a non-homogeneous environment; study of potentialities of the presence of living systems in spite of the low surface temperature - availability of tholins (laboratory analogues of atmospheric organic aerosols) which mimic accurately Titan's aerosols, and the determination of their spectroscopic and optical properties; development of new photochemical models, and determination of new kinetic data of importance for modelling Titan's chemistry. Very recently, new experimental simulations have been carried out to study more specifically the role of the presence of $\mathrm{CO}$ in the chemical evolution of gas mixtures, simulating the behaviour of Titan's stratosphere and the chemistry of O-compounds. The obtained results show that the main O-containing organics in Titan's atmosphere should be oxirane (ethylene oxide) contrary to what was included until now in photochemical models. In addition, several new spectroscopic determinations in the UV and IR ranges have been achieved in particular on polynes compounds and nitriles with unsaturated carbon chains, essential for interpreting the observational data of Titan in these spectral ranges and constructing reliable photochemical models of its atmosphere. These new data will be reviewed and the exobiological consequences will be discussed, in the frame of the arrival of the CassiniHuygens mission in the Saturn system in 2004.

\section{Preparation for a Manned Mission to Mars}

\section{Human Mars mission simulations and exobiology O. Angerer, D. Schmitt, F. Ongaro \\ European Space Agency, ESA/ESTEC, MSM-GAL Keplerlaan 1, P.O. Box 2992200 AG Noordwijk, ZH, The Netherlands, \\ E-mail: oliver.angerer@esa.int}

Recently a new programme for planetary exploration, called Aurora, has been initiated at the European Space Agency (ESA). This programme will provide a long-term perspective for the robotic and human exploration of the solar system, with the main focus on Mars. A further goal of the programme is the technological and scientific preparation that will enable the implementation of the long-term plan. Next to robotic missions and specific technology studies, a major research element of the preparatory activities are human Mars mission simulations. These will be performed using dedicated simulators in a laboratory setting, as well as facilities in partially Martian analogue environments, like Antarctica. These simulations will not only involve experts in medicine, psychology, life support systems and habitability, but also exobiologists. The involvement of exobiologists can be divided into two different aspects. The scientific side: especially in analogue environment settings, exobiological science will be advanced through research performed in these settings. This will improve our general knowledge and thus the chances of success for the robotic and eventually human search for life on Mars. The technological side: for humans to perform exobiological research on Mars, sample handling, preparation and analysis concepts have to be developed, that fulfil planetary protection requirements, allow maximal scientific knowledge gain and ensure the safety of the crew at the same time. These activities, while providing essential information for a future Mars mission, will also produce many applications here on Earth, in areas like microbial monitoring and detection, disease control technologies, tele-science, etc.

\section{Solar energetic phenomena and radiation hazards to biological systems \\ A. Hanslmeier \\ Institute of Geophysics, Astrophysics and Meteorology, University of Graz, Universitätsplatz 5 A-8010 Graz, Austria, E-mail: arnold.hanslmeier@uni-graz.at}

Our Sun is an average star and its overwhelming importance for life on Earth is well known. In this review we will consider different phenomena on the Sun that can be summarized as solar activity phenomena. The solar activity varies with a period of about eleven years. It is related to the generation and transformation of magnetic energy into kinetic energy, which again produces magnetic energy. Studies of the solar dynamo, which explains the periodic occurence of these phenomena, have shown that the solar activity cycle could have phases of irregularities. One very well known period of low solar activity was the Maunder Minimum in the second half of the seventeenth century, which is often associated with a period of extremely cold winters. Therefore, long-term changes of solar activity could influence the Earth's climate. However it is extremely important to study short-term variations, which are accompanied by the emission of dangerous shortwave radiation, and energetic particles, which can be hazardous to manned space missions and cause damage to satellites by surface charging and other effects. We will review the processes on the Sun that are the cause of such energetic eruptions. It will also be shown that today there are worldwide efforts to monitor the Sun continuously. The main aim of solar physics is to understand the physical mechanisms of these phenomena and the interaction with the Earth's space environment. Examples of hazardous events in the past are given and a short discussion of the effects of short-wave radiation and energetic particles on biological systems that are exposed in space. It becomes evident that solar activity must be taken into account for manned (and also unmanned) space missions, becoming extremely important for long-term missions (e.g. stays at the ISS, manned flight to Mars). Some attempts to provide a shielding are reviewed. The influences of the so-called space weather are also discussed for possible landings on Mars.

\footnotetext{
Human missions to Mars and astrobiology, both sides of a coin G. H. Horneck Institute of Aerospace Medicine, Radiation Biology Section, DLR, Linder Höhe D-51147 Köln, Germany, E-mail: gerda.horneck@dlr.de After the realization of the International Space Station (ISS), human exploratory missions to Moon or Mars, i.e. beyond low Earth orbit (LEO), are widely considered as the next logical step of peaceful cooperation in space on a global scale. Besides the human desire to conquer the outer limits of habitability, human exploratory missions are driven by several aspects of science, technology, culture and economy. Mars is currently considered as a major target in the search for life beyond the Earth. Therefore the search for morphological or chemical signatures of life or its relics is one of the primary and most exciting goals of Mars exploration. ${ }^{1,2}$ In addition to exo/astrobiology, disciplines like geology, mineralogy, and atmospheric research play a central role in the scientific exploration of Mars. The presence of humans on the
} 
surface of Mars will substantially increase this research potential, e.g., by supporting deep surface drilling and by in-situ analysis of samples of astrobiological interest. On the other hand, the import of internal and external microorganisms accompanying inevitably any human mission to Mars, or purposely as part of a bioregenerative life support system needs careful consideration with regard to planetary protection purposes. Therefore, before planning any human exploratory mission, the critical issues concerning human health and well being ${ }^{3}$ as well as protection of Mars in its pristine condition need to be jointly investigated. ${ }^{4}$

\section{References}

${ }^{1}$ Brack, A., B. Fitton and F. Raulin. Exobiology in the Solar System and the Search for Life on Mars, ESA/ESTEC, Noordwijk (1999), ESA SP-1231.
${ }^{2}$ Westall, F., Brack, A., Hofmann, B., Horneck, G., Kurat, G., Maxwell, J., Ori, G. G., Pillinger, C., Raulin, F., Thomas, N., Fitton, B., Clancy, P., Prieur, D., Vassaux, D. An ESA study for the search for life on Mars, Planet. Space Sci. (2000), 48, 181-202.

${ }^{3}$ Horneck G., Facius R., Reichert M., Rettberg P., Seboldt W., Manzey D., Comet B., Maillet A., Preiss H., Schauer L., Dussap C. G., Poughon L., Belyavin A., Reitz G., Baumstark-Khan C., Gerzer R., HUMEX, a Study on the Survivability and Adaptation of Humans to Long-Duration Exploratory Missions, ESA-SP (in press).

${ }^{4}$ Horneck G, Facius R, Reitz G, Rettberg R, Baumstark-Khan C, Gerzer R Critical issues in connection with human missions to Mars: protection of and from the environment, Acta Astron. (2001), 49, 279-288.

\section{Permafrost Astrobiology}

\section{Permafrost experiment in the frame of BIOPAN project \\ A. L. Kholodov}

Institute of Physical, Chemical and Biological problems of Soil Science, Russian Academy of Science, 142290, Puschino, Institutskaya Str. 2, Moscow region, Russia, E-mail: akholodov@issp.serpukhov.su

The main goal of the project is to test the resistance of microbial communities, preserved in a frozen state, to space environment at four levels (quantity, quality, molecular, metabolic). We native two permafrost samples of different age (from modern to 3 million years old) with known community of paleo-microorganisms (the number of viable cells, their biodiversity, DNA structure and metabolic activity in frozen/thawing state) to be exposure for Space environment. Samples were taken from ice-reach permanent frozen deposits from North-East of Russia. When the satellite with samples returns to Earth, and the main environmental parameters of such travel are known, we will look once more for the same microbial characteristics. As a result we hope to understand if Space travel is fatal for permafrost microorganisms within the frozen ground, which groups are more resistant and still metabolically active, the part of DNA damages cells could repair. The BIOPAN is a pan-shaped, multiuser experiment facility intended to support research on the effects of cosmic condition on biological and material samples. This facility is attached to the carrier satellite, allowing the experiments to be exposed to vacuum and other space conditions. The parameters of the flight are the following: duration of flight: fourteen days; altitude: about $400 \mathrm{~km}$; temperature of experiment coupled to the carrier plate, heaters switched off: temperature variations from $-35^{\circ}$ to $+30^{\circ} \mathrm{C}$; pressure/vacuum: in orbit, the pressure may drop to $0.133 \times 10^{-6} \mathrm{kPa}(1 \mu \mathrm{m} \mathrm{Hg})$. Rate of pressure changes: maximally $1.333 \mathrm{kPa} / \mathrm{s}(1 \mathrm{~cm} \mathrm{Hg} / \mathrm{s})$. The exact level of space vacuum is not measured in BIOPAN. The PERMAFROST experiment package is the aluminum container with cylindrical shape, covered by transparent quartz glass. The container includes cameras for the samples.

\section{Life in permafrost}

A. Mamukelashvili(1), D. G. Zvyagintsev(1), D. A. Gilichinsky(2)

(1) Soil Biology Department, Soil Science Faculty, Moscow State University, Vorobyovy Gory, Moscow 119899, Russia, E-mail: angela@ps.msu.ru; (2) Institute of Physical, Chemical and Biological problems of Soil Science, Russian Academy of Science, 142290, Puschino, Institutskaya Str. 2, Moscow region, Russia

Frozen sediments of Antarctica and North-East Siberia of great age (up to 15 million years), collected under the leadership of Dr. David Gilichinsky, were investigated for the presence of living organisms by different microscopy, microbiological and biochemical methods. The total quantity of bacterial cells in Arctic and Antarctic sediments of different lithology and age varied from 107 to 108 cells $/ \mathrm{g} \mathrm{dw}$. The viable fungal mycelium is absent, but in all frozen Antarctic and Siberia permafrost sediments viable fungal spores are present. The total count of fungal spores, obtained by luminescent microscopy varies from hundreds of thousands to some millions cells/gdw. Studied frozen samples were characterized by the presence of invertase and amylase activities and absence of protease activity. The level of aminoacides content was quite high. The frequency and level of amylase activity varied in different samples. Activity of invertase, occurring in $90 \%$ of the total amount of the analysed samples, was only one order of magnitude lower in comparison with activity of invertase in tundra soils. The level of intensity of microbial respiratory activity in the Antarctic sediments was comparable with the same index in tundra soils. Additions of glucose to the samples resulted in decrease of respiratory activity of microbes in sediments. It was established that in the presence of oxygen the intensity of carbon dioxide emission is much lower than that in anaerobic conditions. In argon atmosphere the quantity of carbon dioxide produced by microbes was more than two orders higher than in aerobic conditions. It was shown that denitrifying bacteria were unequally distributed in Antarctic sediments. In some cases correlation between the intensity of the nitrifying activity and the total amount of bacteria, accounted for by luminescent microscopy took place. The resistance of permafrost microorganisms to high temperature was investigated. Some of the bacterial and fungal strains of Arctic and Antarctica permafrost showed growth on nutrient media after $3 \mathrm{~h}$ exposition of permafrost samples at $180{ }^{\circ} \mathrm{C}$. They are non-spore-forming bacterial species of genera: Arthrobacter, Micrococcus, Rhodococcus, Flavobacterium, and fungi of genera: Aspergillus, Cladosporium, Penicillium. It is surprising, but even after $800^{\circ} \mathrm{C}$ (SEM method) the fragments of biogel of $50-200 \mathrm{~nm}$ in size were observed, like the similar objects which were found in Martian meteorite ALH-84001!

\section{Life below freezing point \\ E. M. Rivkina}

Institute of Physical, Chemical and Biological problems in Soil Science, Russian Academy of Sciences, 142290, Puschino, Institutskaya Str. 2,

Moscow region, Russia, E-mail: rivkina@issp.serpukhov.su

Significant numbers of viable bacteria are known to be present in permafrost which is 2 to 3 million years old in the Arctic and in permafrost which is probably older in Antarctica. The permafrost microbial community has been described as a community of survivors and this survival state is the result of the combination of cold temperature, desiccation and starvation. At subzero temperatures for periods as long as 2-3 million years in the frozen soils, the rates of microbial metabolic activity would be expected to be extremely low. Permafrost may represent an example of environment where survival of certain life forms continues, and the stringency of the environmental conditions is such that the continuous evolution of successful adaptations is hindered. Any astrobiological models need the answer how long Life might be preserved. We have to establish the principal mechanism of cell's behaviour in permafrost conditions, that probably might work for billions of years. The ecological significance of the metabolic state of permafrost bacteria becomes evident when we consider a key question regarding viable ancient bacteria in permafrost whether they are active in permafrost 
environment or they are in a suspended 'dormant' state. This is why the mechanisms for microbial survival during the both long-term anabiosis or low metabolic state must be studied. The permafrost soil is known to contain unfrozen water. Unfrozen water films make metabolic reactions and mass transfer of microbial metabolic end-products possible within the permafrost, preventing the cell's biochemical death. The obtained results on microbial metabolic activity are a perspective for the newly emerging field of exobiology. Metabolically active chemolithotrophic psychrotolerant bacterial community within permafrost represents an extraterrestrial model for potential life forms with their unique mechanisms to assimilate $\mathrm{CO}_{2}$ and other compounds that might be found in frozen subsurface environments on cryogenic planets without free oxygen, inaccessible organic matter and liquid water near zero.

\section{Possible mechanisms of bacterial survival in terrestrial permafrost as analogues of Martian environment \\ V. Soina}

Soil Science Department, Moscow Lomonosov State University, Vorobyovy Gory, Moscow, Russia, 119899,E-mail:soina@yandex.ru Nowadays, studies of biological activity of subsoil layers suppose that mostly bacteria populate deep terrestrial permafrost sediments. It is known that bacteria in model experiments can survive deep freezing and subsequent thawing, but survival capacity under freezing depends on the cell concentration, the age of the bacterial culture, presence of cryoprotectors and medium composition. Results presented here lead to the assumption that in Earth permafrost sediments with various groups of bacteria and different types of cell walls preserve their viability to a high extent due to the following factors: initially high structural resistance of bacterial cells to freezing, high concentration of the cells in microbial communities, the important role of organo-mineral complex of deposits as cryoprotector and defence against mechanical damage of the cells by ice, physicochemical processes under freezing of deposits (concentration of salt solutions, transfer of liquid moisture, ions, vapor and gases), constant low temperatures in frozen sediments. In these conditions bacterial cells can accumulate osmoprotectants that can serve also as cryoprotectors inside the cells and perform the following functions: stabilize the membranes and intracellular structures, prevent the reduction of cell volume to the critical state because of water removal, prevent freezing of the cytoplasm. The specific ultra structure of the cells as viewed in electron microscopy in situ is characteristic for the dormant state of bacterial cells and may reflect different levels of metabolic activity reduction down to profound rest, and also show their ability to resume vital functions after thawing. Thus, the natural polidisperse heterophase systems as Earth subsoil sediments, accompanied with constant low temperature in permafrost, provide a unique stability to cell structures and as a result preservation of viability of various bacteria, that suppose possible success in search of bacterial life signs in Martian environments.

\section{Viable green algae and cyanobacteria within terrestrial permafrost: implication for exobiology T. A. Vishnivetskaya, E. A. Vorobyova, D. A. Gilichinsky Institute for Physical, Chemical and Biological problems in Soil Science, Russian Academy of Sciences, 142290 Puschino, Institutskaya Str. 2, Moscow Region, Russia, E-mail: Tatiana.vishnivetskaya@Vanderbilt.Edu}

The permafrost is a unique sub-freezing environment that serves as terrestrial model of Mars and other cryogenic space bodies. The main advantage of the permafrost in comparison to simulated cryogenic systems is age. Thus, studying of ancient organisms from permafrost leads to greater understanding of the possibilities and requirements for life outside the Earth. There is an opinion, that cyanobacteria and unicellular green algae were the first microorganisms which performed oxygen photosynthesis, and that they created the Earth's atmosphere. Cyanobacteria and green algae build organic substances from $\mathrm{CO}_{2}$ and water during the process of photosynthesis, and cyanobacteria are also able to fix nitrogen. Furthermore, cyanobacteria can harvest light almost at all wavelengths owing to phycobilisomes, which are light harvesting antenna that can change their structure to achieve better light harvesting. It is well known, that microalgae can exist in different extreme environments. The presented study shows that cyanobacteria and green algae do survive within terrestrial permafrost during prolonged periods of time. Diverse groups of viable cyanobacteria (26 strains) and green algae (54 strains) were isolated from Arctic permafrost. Among them are Oscillatoria, Phormidium, Nostoc, Anabaena, and Chlorella, Chlorococcum, Chodatia, Stichococcus, Pseudococcomyxa, Scotiellopsis, Mychonastes. The age of the oldest strata where they were discovered is 2-3 million years. Unicellular green algae of Mychonastes and Pedinomonas isolated from Antarctic permafrost preserved their cells at $-23{ }^{\circ} \mathrm{C}$ during 170 thousand years. Obviously, they do not thrive in such cold and dark environments but they preserve cell structure and properties and revive photosynthetic activity as soon as light and water become available. Permafrost algae were resistant to simulated natural stresses of cryogenic character as overcooling, freezing, freezing-thawing, desiccation.

\section{Astrobiological view on the polar permafrost as life support ecosystem}

E. Vorobyova(1), G. Osipov(2), A. Filimonova(1),

A. Mamukelashvili(1), N. Bolshakova(2), I. Yaminsky(2)

(1) Soil Biology Department, Moscow State University,

Vorobyovy Gory, Moscow 119899, Russia; (2) Chemical Department, Moscow State University, Russia,E-mail:lenav@ps.msu.ru

Natural environments seem to be intolerable and abolishing for a great majority of microorganisms. Such paradoxical claim not so reveals the imperfection of our knowledge as much as the presence of some scientific misunderstandings and prejudices. The diversity of terrestrial macro- and microenvironments is a reason and consequence of spreading life energy fluxes that reveal and improve biodiversity potentialities. Geological prehistory and the level of subzero temperatures in polar terrestrial permafrost should be the main factors for system development. After millions of years in a frozen state the presence of different biomarkers (carbohydrates, aminoacids, lipids, biogases, pigments, enzymes) as well as high procaryote and eucaryote biomasse content comparable with biomasse abundance in terrestrial soils can be easily recognized in polar permafrost. But considerable dormancy is the main feature for Antarctic subsurface communities. Microbial communities in permafrost samples were investigated by in-situ techniques of lipid microbial biomarkers analyses. Gram-positive bacteria were marked as dominants in most cases, and biodiversity was rather high. Microbial communities inhabiting ancient permafrost sediments demonstrate high stability to the influence of the temperature, high radiation or high concentration of hydrogen peroxide. The investigations provide new information concerning the in situ morphological diversity of prokaryotic and eucaryotic cells in long-term permafrost and ground ice (epifluorescence microscopy, SEM, AFM). An important task was to find features that could be used as criteria for direct cells indication in natural heterogenious environments. It was shown that atomic force microscopy and X-ray analysis could be useful for this purpose. Studies of life in terrestrial permafrost in comparison with other data from environmental ecology gives strong evidence that microbial life on this planet is ubiquitous. Natural environments, and polar subsurface in particular, give a good opportunity for verifying the methodological approaches and instrumental devices for search for life beyond the Earth. 
Methanogenesis as a model process for extreme habitat development: process studies in the active layer of different permafrost sites

\section{Wagner}

Alfred Wegener Institute for Polar and Marine Research, Telegrafenberg A 43, 14469 Potsdam, Germany, E-mail:

dwagner@awi-potsdam.de

Permafrost soils, which cover about $25 \%$ of the Earth's land surface, are continuously frozen throughout the year. Only the active layer thaws during the short arctic summer. During this time methane is produced in the anoxic soil layers and subsequently emitted to the soil near atmosphere with an average emission rate of about $80 \mathrm{mg} \mathrm{CH}_{4} \mathrm{~m}^{-2} \mathrm{~d}^{-1}$. Not all methane will be released to the atmosphere but will be oxidized by methanotrophic bacteria in the oxic soil layers. The soil microorganisms, which are responsible for the methane production and oxidation is highly specialized microbes. The methanogenic archaea (former archaebacteria)- regarded as strictly anaerobic microbes - are considered as one of the first organisms who settled our planet under extreme conditions. They transform simple substances such as acetate and methanol into methane. Most species can even live completely lithoautotroph, without any organic substances, only with hydrogen and carbon dioxide. The methane-oxidizing bacteria, which are able to reduce up to $70 \%$ of the formed methane to carbon dioxide, conclude the methane cycle. These species have adapted themselves over thousands of years to the extreme temperatures of their cold and ice rich habitats. Investigations show that even at soil temperatures $<0{ }^{\circ} \mathrm{C}$ methanogenesis takes place. Under these temperature conditions unadapted organisms are normally inactive or even die. The so-called psychrophilic microbes have a temperature optimum below $15^{\circ} \mathrm{C}$, while they are likely to die above $20^{\circ} \mathrm{C}$. Consequently, the isolated microbes are optimally adapted to the conditions of the terrestrial permafrost. The methanogenic archaea are even growing at very low temperatures, which prevail during the back freezing of the soils in autumn, continuously producing methane that gets into the active layer and will be released in the thawing phase of the following spring. In addition, modern methods like the fluorescence-in-situ-hybridization (FISH) is used to examine the composition of the permafrost soil biocoenosis. These studies help us to comprehend the adaptation and tolerance of microorganisms to the cold environment of permafrost. Furthermore, our comparative system studies provide a contribution to an understanding of the cryosphere on Earth and other extraterrestrial permafrost habitats. Since the terrestrial permafrost areas show similar morphological structures like Martian surface, it could be assumed that their development is based on comparable processes. Microbiological and molecular ecological studies in combination with environmental, pedochemical and physical analyses can give insights into early stages of life in extreme ecosystems.

\section{Bacterial diversity and DNA survival in ancient permafrost and its implications for finding nucleic acid based extraterrestrial life \\ E. Willerslev}

Department of Evolutionary Biology, Zoological Institute, University of Copenhagen and Center for Planetary Science, Niels Bohr Institute,

Danish Space Research Institute, Universitetsparken 15, DK-2100 Ø,

Denmark, E-mail: ewillerslev@zi.ku.dk

Studies of bacteria diversity in permafrost (permanently frozen soil) have been limited to identification of viable cultures. We now demonstrate well-preserved environmental bacteria DNA in ancient Siberian permafrost between 5-7,000- and 300-400,000-years-old confining the oldest DNA reproducibly obtained from any geological setting. Bacteria diversity was found to decline with time and no putative DNA could be obtained from million-year-old samples. DNA from non-spore-forming bacteria appeared superior in preservation to that of spore-formers. Our results have implications for finding long-term preserved microbes in extreme subsurface environments such as rocks, ice cores as well as nucleic acid-based extraterrestrial life-forms on for example Mars, as it seems unlikely that microbes can have survived these adverse conditions for hundreds of thousand to million or billion of years as resting cells.

\section{Exploration of Planetary Subsurfaces}

\section{The planet within: exploring cave and subsurface habitats on Earth and Mars \\ P. J. Boston}

Department of Earth and Environmental Science, New Mexico Institute of Mining and Technology, New Mexico Tech, Socorro NM 87801, USA,E-mail: pboston@complex.org

The subsurface of Earth shows us a very different planet from the familiar one we see on its surface or in its oceans. Unique microorganisms perform myriad chemical and mineralogical transformations and live in habitat types including near-surface caves, aquifers, very deep-sealed voids, multi-km deep mine adits, and minute fractures within igneous masses. Similarly, the subsurface of Mars undoubtedly shows a very different character from the surface conditions. We have long suggested that the Martian subsurface should be a major focus for exobiological investigations. Our field research in caves in many geological settings (e.g. lavatubes, karst, ices, etc.) has led us to develop suites of detectable biosignatures that accompany each fundamental geological cave setting and available energy sources. Recently, we have begun to correlate properties of some surface oxides and rinds (e.g. desert varnish) to related processes and microbial activities in caves under the same desert environments. These correlations may have utility for site selection and future biomarker detection on the desert planet Mars. Cross-correlating different data sets (e.g. imaging, chemistry, micromineralogy, biomolecule detection, and molecular phylogenetic analyses) at the precise same sampling site has proven critical to unambiguously distinguish biogenic materials from those where biology is incidental or the result of abiological processes. Matching modern, active cave microbial communities with similar well-preserved ancient cave microbiota remains has also contributed to unravelling the complex story of subsurface life. We believe that this need for data autocorrelation is an important ingredient for mission planners to take into account. Subsurface environments may be more common on rocky, terrestrial and icy planets than we have thought in the past. Indeed, our burgeoning knowledge of Earth's subsurface biosphere is less than two decades old. Such biota may not leave the types of traces (e.g. free oxygen atmospheres) that we associate with life as we know it on our planet. Telescopic, life-detection indicators for extra-solar planet-finder type missions now under conceptual development may seriously underestimate the total number of planets with biospheres.

\section{Organic and inorganic signatures in Mars ground} and underground, one of the goals for SAM

M. Cabane(1), P. Coll(2), G. Israel(1), F. Raulin(2), H. Niemann(3), P. Mahaffy(3), R. Sternberg(2), A. Person(4), C. Rodier(5),

W. Brinckerhoff( 6 )

(1) Service d'Aéronomie du CNRS, IPSL, Univ. Paris 6, Paris,

France, E-mail:michel.cabane@aero.jussieu.fr; (2) Laboratoire Interuniversitaire des Systèmes Atmosphériques, Universités Paris 7 et Paris 12, Créteil, France; (3) NASA Goddard Space Flight Center, Greenbelt, MD, USA; (4) Laboratoire de Géologie Sédimentaire, Univ. Paris 6, France; (5) Laboratoire de Synthèses et Réactivités, Univ. de Poitiers, France; (6) Applied Physics Lab, The John Hopkins Univ., $M D, U S A$

In its history, Mars may have seen life developing in zones analogue to Earth's biosphere. Future exploration, in the next decade, will be, in part, devoted to investigations on extinct or extant traces of life, or prebiotic chemistry that could have existed. The 2009 Smart Lander 
drilling system could attain depths of 1 , possibly $10 \mathrm{~m}$, which would permit the sampling of zones where organic remnants may have been preserved from oxidation. This was not the case for Viking, which sampled mainly in the first centimeters. To search for organic signatures, we develop new solutions in the experiment that will be presented in the frame of a US/French proposal, SAM (Sample Analysis at Mars), devoted to in-situ atmospheric, ground, and underground chemical and isotopic analysis. Crucial information on the past Martian atmosphere and the possible apparition of life, may be obtained from the mineralogical composition of the samples. To assert the presence of inorganic relevant species (carbonates, salts, clays, etc.), the presence of which may be correlated with life-sustaining sites, SAM ovens will permit heating of the samples $\left(\operatorname{Tmax}=1100^{\circ} \mathrm{C}\right)$ to observe the release of structural gases $\left(\mathrm{CO}_{2}, \mathrm{H}_{2} \mathrm{O}, \mathrm{SO}_{2}\right.$, etc. $)$ at defined temperatures. Minerals may be present under the form of biomineralizations, inorganic minerals whose behaviour with the temperature may be different to minerals grown in abiotic conditions. Moreover, heating of the sample at moderate temperatures will permit us to observe gases adsorbed on mineral grains, especially $\mathrm{H}_{2} \mathrm{O}_{2}$ which may be the main atmospheric oxidant, and $\mathrm{H}_{2} \mathrm{O}$, which will help to refine the Martian water budget. The organic molecules that may have beeen preserved (kerogens, amino acids, carboxylic acids, etc.) may be too refractory to be detected by pyrolysis at relatively low temperatures ( $\mathrm{T} \leftarrow 500{ }^{\circ} \mathrm{C}$ on Viking); the high temperatures that we intend to attain in SAM's ovens will allow us to search for these molecules. The most refractory molecules, especially those occurring after oxidation processes, in the first layers of the ground, will be identified using the derivatization technique which preserves the structural information, but transforms such molecules into analysable ones (this technique will also permit us to observe fragile molecules). In the proposed SAM experiment, the gases produced after heating, pyrolysis, and derivatization processes, will be analysed, using the coupling of Gas Chromatography and Mass Spectrometry), both techniques that we have developed in our laboratories in the frame of previous space experiments. These techniques will also be used to perform measurements of the atmospheric isotopic composition. This complementary part of the experiment, as well as the analysis of the solid sample by laser ablation and time of flight spectrometry, will be mainly performed by the US side of SAM.

\section{Robotic astrobiology - the need for sub-surface penetration of Mars}

A. Ellery(1), A. Ball(2), C. Cockell(3), P. Coste(4), D. Dickensheets(5), H. Edwards(6), H. Hu(7), C. Kolb(8,13), H. Lammer(8), R. Lorenz(9), G. McKee(10), L. Richter(11), A. Winfield(12), C. Welch(1)

(1) School of Engineering, Kingston University, London, UK, E-mail: a.ellery@kingston.ac.ukc.s.welch@kingston.ac.uk,

(2) Planetary and Space Sciences Research Institute, Open University, Milton Keynes, UK, E-mail: a.j.ball@open.ac.uk; (3) British Antarctic Survey,Cambridge,UK,E-mail: csco@bas.ac.uk; (4) Mechanical Engineering Department, ESA/ESTEC, The Netherlands, E-mail: pierre.coste@esa.int; (5) Department of Electrical and Computer Engineering, Montana State University, Nozeman, Montana, USA E-mail: davidd@ee.montana.edu; (6) Department of Chemical and Forensic Sciences, University of Bradford, Bradford, UK, E-mail: h.g.m.edwards@Bradford.ac.uk; (7) Department of Computer Science, University of Essex, Colchester, UK, E-mail:hhu@essex.ac.uk; (8) Department of Extraterrestrial Physics, Space Research Institute, Austrian Academy of Sciences, Schmiedlstr. 6, A-8042 Graz, Austria, E-mail:helmut.lammer@oeaw.ac.at; (9) Lunar and Planetary Laboratory, University of Arizona, Tucson, Arizona, USA,

E-mail: rlorenz@lpl.arizona.edu; (10) Department of Computer Science, Cybernetics \& Electronic Engineering, University of Reading, Reading, UK, E-mail: Gerard.McKee@reading.ac.uk; (11) Institute of Space Simulation, DLR, Cologne, Germany, E-mail:

lutz.richter@dlr.de;(12) Intelligent Autonomous Systems

Laboratory, University of the West of England, Bristol, UK,

E-mail: Alan.Winfield@uwe.ac.uk; (13) Institute for Mineralogy and Petrology, University of Graz, Universitätsplatz 2,

A-8010 Graz, Austria

Recent interest in the astrobiological investigation of Mars (and eventually Europa) has culminated in the only planned astrobiology-focussed robotic mission to Mars - the UK-led European Beagle 2 mission to be carried to Mars by the ESA Mars Express spacecraft in 2003 Beagle 2, like most proposed Mars missions will be primarily investigating the surface and near-surface environment of Mars. However, the results from the Viking Mars lander (1976) indicated that the Martian surface is saturated in peroxides and super-oxides, which would rapidly degrade any organic material. Furthermore, recent models of gardening due to meteoritic impacts on the Martian surface suggest that the depth of this oxidising layer could extend to depths of $2-3 \mathrm{~m}$. Given that the discovery of organic fossilised residues will be the primary target for astrobiological investigation, this implies that future robotic astrobiology missions to Mars must penetrate to below these depths. The need to penetrate into the sub-surface of Mars has recently been given greater urgency with the discovery of extensive water icefields as little as $1 \mathrm{~m}$ from the surface. We review the different technologies that make this penetration into the sub-surface a practical possibility on robotic missions. We further briefly present one such implementation of these technologies through the use of ground-penetrating moles - the Vanguard Mars mission proposal.

\section{Martian Cryoscout and contamination detection} A. J. Hansen

Department of Evolutionary Biology, Zoological Institute, University of Copenhagen and Center for Planetary Science, Niels Bohr Institute, Danish Space Research Institute, Universitetsparken 15, DK-2100 Ø. Denmark,E-mail: ajhansen@zi.ku.dk

A microarray chip based on PNA (peptide nucleic acid) capture probes for detection of $18 \mathrm{~S} / 16 \mathrm{~S}$ rDNA contaminating sequences is currently being developed for the Martian Cryoscouts. Its purpose is to detect possible contaminating DNA/RNA brought by the Cryoscouts from earth. The chip may also detect possible remains of nucleic acid-based life forms in the Martian ice caps.

\section{Search for traces of water by Atomic Force Microscopy}

A. Kempe(1), W. Altermann(2), W. M. Heckl(1)

(1) Institut für Kristallographie und Angewandte Mineralogie, LMU, Theresienstr. 41, D-80333 München, E-mail: andre.kempe@nanogeology.de; (2) Institut für Allgemeine und Angewandte Geologie, LMU, Luisenstr. 37, D-80333 Munich In the search for aqueous habitats on Mars direct proof of (ancient) flowing water is still lacking although remote sensing has provided indications for young fluvial systems. ${ }^{1,2}$ To demonstrate that such proof can be given we analysed surface marks on recent terrestrial sand grains with an atomic force microscope (AFM). Surface textures of sand grains have been used before to distinguish between aeolian, aquatic and glacial transport, the scanning electron microscope (SEM) being the most prominent tool used. ${ }^{3-9}$ The interpretation of results greatly relied on the spectator experience however, since SEM data are mostly descriptive. We tried an experimental verification that quantitative 3 d-analysis of sand grain surfaces by AFM can numerically distinguish between aeolian and aquatic transport in sedimentary deposits on Earth. The surfaces of natural quartz grains as well as olivine, plagioclase and pyroxene sands of known origin have been imaged by AFM, each image providing a $3 \mathrm{D}$ map of the mineral surface. Image processing was applied to find the fringes of characteristic etch pits that are typical for aqueous sedimentary grains. The fringes of the pits were then segmented in straight lines. The length and orientation of each segment was measured and analysed statistically, providing a tool that is sensitive to the existence of surface structures with parallel orientation on the grains. Comparison of aeolian quartz to aquatic quartz shows that the method can be used to differentiate between the two environments on Earth. Furthermore etching structures on other mineral grains relevant to the Martian geology were successfully detected by AFM analysis. Assuming that Martian aqueous sand grains exhibit similar patterns as quartz and other mineral grains on Earth, we conclude that similar surface structures should be detectable in aqueous sedimentary environments that may have existed on mars. 


\section{References}

${ }^{1}$ Malin, M. C. and Carr, M. H. Groundwater formation of Martian valleys. Nature (1999), 397, 589-591.

${ }^{2}$ Malin, M. C. and Kennet, S. E. Evidence for Recent Groundwater Seepage and Surface Runoff on Mars. Science (2000), 288, 2330-2334.

${ }^{3}$ Carter, J. M. L. An application of scanning electron microscopy of quartz sand surface textures to the environmental diagnosis of Neogene carbonate sediments, Finestrat Basin, south-east Spain. Sedimentology (1984), 31, 717-731.

${ }^{4}$ Culver, S. J., Bull, P. A., Campbell, S., Shakesby, R. A. and Whalley, W. B. Environmental discrimination based on quartz grain surface textures; a statistical investigation. Sedimentology (1983), 30, 129-136.

${ }^{5}$ Fortuin, A. R. Late Ordovician glaciomarine deposits (Orea Shale) in the Sierra de la Albarracin, Spain. Paleogeography, Paleoclimatology, Paleoecology (1984), 48, 245-61.

${ }^{6}$ Higgs, R. Quartz grain surface features of Mesozoic-Cenozoic sands from the Labrador and West Greenland continental Margins. J. Sedim. Petrol. (1979), 49, 599-610.

${ }^{7}$ Krinsley, D. H. and McCoy, F. W. Significance and origin of surface textures on broken sand grains in deep-sea sediments. Sedimentology (1977), 24, 857-862.

${ }^{8}$ Krinsley, D. H. and Doornkamp, J. C., eds. Atlas of quartz sand surface textures (Cambridge University Press, 1973).

9 Krinsley, D. H. and Takahashi, T. The surface textures of sand grains, an application of scanning electron microscopy. Science (1962), 138, $1262-1265$

\section{Melting probes as a means to explore planetary glaciers and sub-surface oceans \\ N. I. Kömle}

Department for Extraterrestrial Physics, Space Research Institute, Austrian Academy of Sciences, Schmiedlstr. 6, A-8042 Graz, Austria, E-mail:norbert.koemle@oeaw.ac.at

On many planetary bodies thick layers of frozen volatiles cover the surface. Examples are: The polar ice caps of Earth and Mars, consisting of $\mathrm{H}_{2} \mathrm{O}$-ice and a mixture of $\mathrm{H}_{2} \mathrm{O}$ - and $\mathrm{CO}_{2}$-ice, respectively; Neptune's satellite Triton, whose polar areas are composed of nitrogen $\left(\mathrm{N}_{2}\right)$-ice, and the icy satellites of Jupiter (in particular Europa), which most probably host liquid layers ('oceans') below their ice crusts. Access to deeper parts of the ice layers is of ultimate importance for understanding the climatological history of the respective bodies, as well as for exobiological aspects. Icy layers often 'grow' continuously in the course of the planetary history - and thus should contain key information on the surface conditions in the past, including the possibility of finding find remnants of extinct primitive life. In contrast to solid rocky surfaces (where the application of heavy and power-demanding drilling and hammering devices is the only possibile way to access their interior), ice layers could be explored by much simpler means, namely melting probes. We review several concepts for such devices, the state of art in the development, and discuss possible applications for the exploration of the Mars polar areas and the ice crust and oceans of Jupiter's satellite Europa, which currently is thought to be a promising target in the search for extraterrestrial life. Estimates for the power consumption expected for such probes and the penetration speed through various ice sheets are also given.

\section{Detection and characterization of ice and water deposits on Mars by means of mutual impedance $(\mathrm{mi})$ probes on surface and subsurface vehicles \\ R. Trautner, R. Grard, F. Simões}

Research and Scientific Support Department, ESA/ESTEC Noordwijk, The Netherlands, E-mail: roland.trautner@esa.int, rtrautne@estec.esa.nl

Mutual Impedance Probes measure the complex permittivity of materials. The measurement principle and its performance have been demonstrated successfully in laboratory and field tests, and flight instruments have been developed for the HUYGENS and ROSETTA lander missions. Most dry materials have very low electrical conductivities, and their relative dielectric constants lie typically in the range from 1 to 5 . In contrast, water is characterized by a much higher permittivity, especially at low frequencies. The permittivities of water, and also its conductivity, are functions of temperature and frequency. Water therefore possesses a clear signature, which enables it to be differentiated from other materials in the Martian environment. In the field of Astrobiology, water is generally seen as one of the most important ingredients that allow the development of life. This is also valid for Mars, where recent research suggests the presence of considerable amounts of water in the Martian subsurface. MI probes can be used for detection and characterization of subsurface ice and water deposits. Rover based MI probes can be used for 2D mapping of ice concentrations in the Martian subsurface, and MI probes on moles or drills can characterize the structure and composition of the subsurface as part of an instrument package and help identify the most promising locations for soil sampling and in-detail investigations. We introduce the design features and measurement performance of MI probes. The expected electrical characteristics of Martian material/ice mixtures are recapitulated. MI probe architectures for surface mapping and subsurface characterization are shown, and strategies for identifying promising sampling locations are discussed. Recent results from laboratory and field measurements are presented, and ongoing development activities at ESTEC are described.

\section{Multisensor Soil Electromagnetic Sounding (MUSES) for Mars exploration}

G. Vannaroni(1), R. Filippini(2), E. Pettinelli(3), M. Storini(1), G. Della Monica(3), R. Di Maio(4), R. Orosei(5), C. Ottonello(2), G. Tacconi(2), S. Orsini(1), S. Pagnan(2), A. Galli(6), G. Schettini(7), A. Menghini(8), A. M. Di Lellis(9), D. Del Vento(1), F. Paolucci(3), A. Cereti(3)

(1) Istituto Fisica Spazio Interplanetario, CNR via del Fosso del Cavaliere 100,00133 Roma, Italy,E-mail: giuliano.vannaroni@, ifsi.rm.cnr.it; (2) Istituto di Studi sui Sistemi Intelligenti per l'Automazione, CNR via dei Marini 6, 16149 Genova, Italy, (3) Dipartimento di Fisica, Universita' Roma Tre, via della Vasca Navale 84, 00146 Roma, Italy; (4) Dipartimento Scienze Fisiche, Universita' di Napoli 'Federico II', via Cintia, 80126 Napoli, Italy; (5) Istituto di Astrofisica Spaziale e Fisica Cosmica, CNR via del Fosso del Cavaliere 100, 00133 Roma, Italy; (6) Dipartimento di Ingegneria Elettronica, Universita' di Roma La Sapienza, via Eudossiana 18, 00184 Roma, Italy; (7) Dipartimento di Ingegneria Elettronica, Universita' Roma Tre, via della Vasca Navale 84, 00146 Roma, Italy; (8) S.Te.G.A., Studio di Tecnologie per la Geologia e per l'Ambiente, Via Monte Bianco 5, Viterbo, Italy; (9) AMDL, via Giovanni Angelini 33, 00149 Roma, Italy

We present a Multisensor Soil Electromagnetic Sounder (MuSES) designed to perform in-situ measurements of the electromagnetic properties of the shallow soil, in the course of future landing missions to Mars. Ground based electromagnetic soundings are strongly recommended to: 1.) select locally a suitable site having the best subsoil features for searching extinct or extant life, before deploying specific biological experiments; 2.) improve, in case of drilling, the target information and optimize the boring operation; 3.) complement the electromagnetic observations carried out by orbital experiments, with a detailed ground truth of the conductivity and dielectric constant of the soil. The experimental package consists of three instruments: (1) A Transient ElectroMagnetic (TEM) system that probes the subsoils apparent resistivity down to several meters. (2) A Ground Penetrating Radar (GPR) able to detect the dielectric/magnetic discontinuities in the subsurface up to a depth of 2-3 meters. (3) A Soil Dielectric Spectroscopy Probe (SDSP), which measures the complex permittivity (conductivity and dielectric constant) of the upper soil layer (within a thickness of some tens of centimeters). The various sensors do not require physical contacts with the soil and are therefore suitable to be installed on a mobile vehicle. The authors describe the rationale of the Multisensor instrumental package, its performance and the improvements, in terms of scientific return, expected from the relevant data fusion. 


\section{Search for Life on Europa and Prebiotic Chemistry in Titan's Atmosphere}

\author{
Acetylene-based pathways for prebiotic evolution \\ on Titan \\ O. Abbas(1), D. Schulze-Makuch(2) \\ (1) Environmental Science and Engineering, University of Texas \\ at El Paso, University Avenue, El Paso, TX 79968-0555, USA, \\ E-mail: abbas101@msn.com; (2) Department of Geological Sciences, \\ University of Texas at El Paso, USA
}

Due to Titan's reducing atmosphere and lack of an ozone shield, ionizing radiation penetrates the atmosphere creating ions, radicals and electrons that are highly reactive producing versatile chemical species on Titan's surface. We propose that the catalytic hydrogenation of photochemically produced acetylene may be used as a simple metabolic pathway by organisms at or near Titan's surface: $\mathrm{C}_{2} \mathrm{H}_{2}+3 \mathrm{H}_{2} \rightarrow 2 \mathrm{CH}_{4}$ Spectral evidence indicates that the product of this reaction, methane, is found to be isotopically lighter than would be expected from Titan's formation theory, and thus may hint toward microbial fractionation. While the acetylene may undergo this reaction, it can also undertake a second route of catalytic self-assembly of three acetylenes to produce benzene under Titan's low temperature conditions: $3 \mathrm{C}_{2} \mathrm{H}_{2}+$ Catalyst $\rightarrow \mathrm{C}_{6} \mathrm{H}_{6}$. A variety of substrates such as active metal surfaces, clay minerals or zeolites could act as surface catalysts. Once benzene is produced, the synthesis of more complex compounds occurs via simple substitution or addition reactions. For example, nitrobenzene could be produced using $\mathrm{NO}_{2}{ }^{+}$, which is formed from ionizing radiation, nitric acid or the oxidation of ammonia: $\mathrm{C}_{6} \mathrm{H}_{6}+\mathrm{NO}_{2}{ }^{+} \rightarrow \mathrm{C}_{6} \mathrm{H}_{5} \mathrm{NO}_{2}$ Nitrobenzene can then be the starting point for the production of even more complex organic molecules, such as amino acids and peptides. Alternatively, acetylene may yield amino acids directly via a conversion of an alkyne to the corresponding aldehyde, which then forms an alpha amino nitrile and eventually an amino acid. The needed higher temperatures for this reaction to occur would mean that this scenario would be tied to the presence of hydrothermal vents and impact craters, or to the deeper subsurface of Titan.

\section{Chemistry in Titan's atmosphere: how the $\mathrm{C} / \mathrm{N}$ and $\mathrm{C} / \mathrm{H}$ ratios of aerosol analogues are correlated to pressure and temperature of laboratory experimental simulation \\ $J$-M. Bernard \\ LISA Université Paris 12 et Paris 7, UMR CNRS 7583, CMC 61 avenue du Général de Gaulle, 94010 Creteil, Cedex, France, \\ E-mail:bernard@lisa.univ-paris12.fr}

The gas present in Titan's atmosphere is leading to organics aerosols under action of the solar radiations and electrons from Saturn's magnetosphere. Many experimental simulations have been realized by irradiating $\mathrm{N}_{2} / \mathrm{CH}_{4}$ gas mixtures with different energy sources, in order to reproduce the chemistry of gas and particulate phases. Until very recently, only one organics remains detected in Titan but never in laboratory simulation: $\mathrm{C}_{4} \mathrm{~N}_{2}$. A full programme of experimental research has been developed at LISA, which permits us to provide a complete identification of a wide range of compounds, similar to those present in Titan's atmosphere, including $\mathrm{C}_{4} \mathrm{~N}_{2}$. The composition of aerosol on Titan is unknown yet, in part due to its complexity. Even if functional groups of analogues have been determined using spectroscopy and pyrolysis, its building molecules are especially difficult to identify. However this chemical composition is a key parameter for Cassini/Huygens experiments and atmospheric modelling. We will present the first results related to the variation of $\mathrm{C} / \mathrm{N}$ and $\mathrm{C} / \mathrm{H}$ ratios with the temperature and the pressure in Titan's atmosphere simulations. These data will allow us to construct photochemical models, in order for them to be more realistic. Then the understanding of the mechanism of aerosols formation on Titan as a function of altitude will be easier.

\section{Schumann resonances as indicators for lightning on Titan}

B. P. Besser(1), K. Schwingenschuh(1), I. Jernej(1), H. U.

Eichelberger(1), H. I. M. Lichtenegger(1), M. Fulchignoni(2)

(1) Space Research Institute, Department of Experimental Space

Research, Austrian Academy of Sciences, Schmiedlstr. 6, A-8042 Graz,

Austria,E-mail:bruno.besser@oeaw.ac.at; (2) Université de

Paris 7 and Observatoire de Paris-Meudon, 2 Place Jussieu,

F-75005 Paris, France

We calculate the eigenmodes of the cavity bounded by Titan's surface and the ionosphere, the so-called Schumann resonances. For the analytical treatment we use a model with a simplified geometry and total-reflecting boundaries. This model is the basis for extended numerical investigations using the Finite Element Method (FEM). We study the influence of non-spherical geometries and more realistic height profiles of the electrical conductivity on the frequency spectrum. The Huygens Atmospheric Structure Instrument (HASI) on the Huygens probe will investigate during its descent the atmospheric structure of Titan below $170 \mathrm{~km}$. The HASI/PWA (Permittivity, Wave and Altimetry) instrument will measure electrical parameters, like electron and ion-electron conductivities. Additionally, it will detect emissions from natural wave phenomena from dc up to $10 \mathrm{kHz}$. Lightning, if it exists, could be detected, and if lightning discharges occur frequently in the atmosphere and the damping is low, Schumann resonances could occur.

Aknowledgement

B. P. Besser acknowledges partial support by a Friedrich-Schmiedlscholarship.

\section{Can evolutionary convergence be tested on Europa? J. Chela-Flores}

The Abdus Salam International Centre for Theoretical Physics, Strada Costiera 11; 34136 Trieste, Italy, E-mail: chelaf@ictp.trieste.it Numerous space missions are being currently planned by the space agencies for the next ten to twenty years. They are intended to explore extensively our own solar system, including planets, satellites, comets and asteroids. A major objective in solar system exploration will be the insertion of appropriate biology-oriented experiments. Earlier we had proposed to extend the range of biomarkers that may be used in the search for microorganisms in our own solar system. The proposed wider range should include universal evolutionary biomarkers. We discuss various reasons for suggesting that this type of research be considered for feasibility studies and, subsequently, for technological development of appropriate landers and submersibles. Various degrees of convergent phenomena are singled out for discussion. In Darwin's theory we consider the role of contingency in the likelihood of the emergence of specific biological properties. We conjecture that the phenomenon of convergent evolution can be extended to the cosmic level. We suggest how such a conjecture can be subjected to experimental verification in the context of solar system exploration. Within the present level of technological development we point out possible experiments to test the conjecture. They are based on direct observation of microorganisms in other environments that are within our reach in the solar system. The special case of missions focussing on the search for microorganisms in the Jovian satellite Europa will be considered.

\section{Laboratory studies on hydrocarbons - application to theoretical models of Titan's atmosphere M.-C. Gazeau, Y. Bénilan, P. Coll, A. Jolly, V. Vuitton, F. Raulin LISA, Laboratoire Interuniversitaire des Systèmes Atmosphériques, CMC, Faculté des Sciences 61, avenue du Général de Gaulle 94010 CRETEIL Cedex, France, E-mail: gazeau@lisa.univ-paris 12.fr \\ To interpret the concentrations of the products measured in Titan's atmosphere and to better understand the associated methane chemistry, theoretical models have been developed..$^{1-3}$ Unfortunately, large dis- crepancies have been found between theoretical and observational data}


concerning the mixing ratio of some compounds in the stratosphere. A critical examination of the chemical scheme included in these models points out some problems regarding: (1) the reliability of the description of critical reaction pathways; (2) the accuracy of kinetic parameters (absorption cross-sections, quantum yields and rate constants). Laboratory experiments can be used to reduce these two sources of uncertainty and then refine the theoretical description of the processes involved in Titan's atmosphere. Thus, we are developing: (1) a programme coupling simple and well-constrained photochemical experiments and theoretical studies in order to improve the restricted part of the chemical scheme. Following the photochemical evolution of a simple gas mixture can provide quantitative data that can be directly used to test the accuracy of the chemical scheme. The first study performed is related to the photocatalytic dissociation of methane by acetylene, a process that has been suggested to explain the concentration of ethane in Titan's stratosphere. (2) UV studies in order to determine fundamental parameters that will be used to feed models. These studies concern polyynes $\left(\mathrm{C}_{2} \mathrm{nH}_{2}\right.$ : compounds of primary interest since they could link the gaseous and the solid phase) and particularly diacetylene $\left(\mathrm{C}_{4} \mathrm{H}_{2}\right)$ as its relative abundance is not well reproduced by theoretical models. We will present the results of these works as well as a future experimental programme dedicated to the simulation of Titan's stratosphere in the most representative conditions (energy deposition, $\mathrm{T}$ and $\mathrm{P}$ ) ever achieved so far. References

1 Yung et al. Astrophys. J. Supp. Series (1984), 55, 465.

2 Toublanc et al. Icarus (1995), 113, 2.

${ }^{3}$ Lebonnois et al. Icarus (2001), 152, 384.

\section{Enhanced hydrogen peroxide formation by oxygen implantation in water ice: An energy source for an Europa biosphere?}

O. Gomis(1), M. A. Satorre(1), G. Leto(2), G. Strazzulla(2)

(1) Departamento de Fisica Aplicada Escuela Politecnica Superior

Alcoy,Univ Politecnica de Valencia, Spain,E-mail: gianni@ct.astro.it ;

(2) INAF Osservatorio Astrofisico di Catania, Via S. Sofia 78 95123,

Catania, Italy

We have studied the formation of hydrogen peroxide under ion implantation of water ice. Thick layers (i.e., larger than the penetration depth of the used ions) of water ice, formed by vapour deposition onto a silicon substrate at 16 and $77 \mathrm{~K}$, have been irradiated with $30 \mathrm{keV}$ single charged ions: $\mathrm{H}, \mathrm{C}, \mathrm{N}, \mathrm{O}$ and Ar. The formation of the 3.5 micron hydrogen peroxide band has been studied by infrared transmission spectroscopy performed in a high-vacuum chamber. We demonstrate that hydrogen peroxide is produced whatever is the used ion, and both at 16 and $77 \mathrm{~K}$. The maximum amount of formed hydrogen peroxide in respect to water ice ranges from $0.2 \%$ for protons to $2 \%$ for $\mathrm{Ar}$ and is independent of the temperature except for oxygen implantation. In this latter case the maximum concentration is $1.5 \%$ at $16 \mathrm{~K}$ and $4.4 \%$ at $77 \mathrm{~K}$. This result cannot be due to the amount of implanted oxygen that changes the composition of the target. Probably implanted oxygen ions act as scavengers of electrons or of atomic and molecular hydrogen, promoting the formation of water molecular fragments. At $77 \mathrm{~K}$ these fragments could rapidly diffuse and induce the formation of larger amounts of hydrogen peroxide. The experimental results will be presented and discussed in the light of the relevance they could have as an energy source for a Europa biosphere.

\section{Estimation on the thickness of the ice crust of Europa A. Kereszturi Department of Physical Geography, Eötvös Loránd University of Sciences, H-1117 Budapest, Pazmany setany 1/C., Hungary, E-mail: krub@freemail.hu}

Based on the images of Europa made by the Galileo spacecraft we measured the shadow lengths and relative heights of topographic structures above the surrounding terrain and the vertical topographic profile of ice blocks to make simple digital topographic terrain models. At a $48 \times 38 \mathrm{~km}$ part of the famous Conamara chaos we measured the relative heights and maximum diameters of the blocks. The distribution shows one typical height which could be related to the original, "undisturbed' thickness of ice. The distribution curve turns off around $2 \mathrm{~km}$ diameter which could be the result of the tilting of originally ' higher than wider' blocks with their longer axis into the horizontal plane, into stable drifting orientation. This causing smaller height, and with the turn-off point we estimated the isometric block diameter and the absolute original ice thickness to be around $2 \mathrm{~km}$ and the thickness of the lower undulating ice matrix only about $0.5 \mathrm{~km}$ at the chaotic terrain. Now we are working on photoclinometric topographic maps corrected with shadow based heights and crustal thickness maps based on Airy isostasy to gain a GIS based automatic method for the analysis of the ice of Europa.

\section{Exploring the unknown world of Europa: The next challenge? \\ J.-L. Josset}

Micro-Cameras and Space Exploration (SPACE-X), 1 rue Jaquet

Droz, 2007 Neuchâtel, Switzerland,E-mail: jean-luc.josset@space-x.ch Except for the Earth, a good candidate to find life in the solar system is Europa. After the development of powerful imaging systems for planetary exploration combining low power, low mass, wide temperature operation and high scientific return, the next challenge seems to be Europa exploration, since it combines all the difficulties. The present imaging systems have been developed and selected for several exploration missions like Mars Express, Rosetta, Smart-1. Although the requirements for these missions are severe, a further step is needed for the Europa mission in terms of power, mass, harsh environment (temperature, pressure, humidity, darkness). A micro-imaging system based on new high micro-technology developments could be implemented in the Europa lander to image the landing site and even inside the Europa deep drill facility to discover the deep unknown world of Europa ocean.

\section{Tidal winds on Titan - measurement goals and} mobility opportunities for future missions

\section{T. Tokano(1), F. M. Neubauer(2), R. D. Lorenz(3)}

(1) Institut für Raumsimulation, DLR, 51170 Köln, Germany, E-mail: tetsuya.tokano@dlr.de; (2) Institut für Geophysik und Meteorologie, Universität zu Köln, D-50923 Köln, Germany; (3) Lunar and Planetary Laboratory, LPL, University of Arizona, Tucson, AZ 85721, USA

Previous climate models of Titan indicated that the wind in Titan's troposphere would be dominated by rather uniform Westerlies and weak Hadley circulation. Recent study shows that Saturn's tide gives rise to much stronger near-surface winds in a north-south direction with a periodical change of wind direction every few days. The Huygens probe may detect the presence of tidal wind, but will not be able to measure its spatial and temporal variation. These winds will affect the precipitation of condensed hydrocarbons and nitriles by meridional transport, so the composition and global distribution of surface deposits of condensed material may be different from those predicted by simple 1-dimensional photochemistry-condensation models. The spatial heterogeneity of surface deposits means that future missions will need to sample different locations to fully characterize Titan's organic inventory, suggesting a mobile (aerial) platform. Such a platform (perhaps a zeppelin) will also be able to make detailed in-situ meteorology measurements, and can exploit the predictable tidal winds to cover large distances without extreme propulsive cost. 


\section{Search for Extra-Solar Terrestrial Planets}

First detection of water in extra-solar planets: A new step in Astrobiology

Cristiano Cosmovici(1), Massimo Teodorani(2), Stelio

Montebugnoli(2), Giuseppe Maccaferri(2)

(1) Istituto Scienze Cosmiche e Planetarie, CNR, Rome, Italy, E-mail: cosmo@ifsi.rm.cnr.it; (2) Istitutodi Radioastronomia, $C N R$, Medicina Bologna, Italy

The ITASEL programme (Italian Search for Extraterrestrial Life) started in 1994 after the discovery of the first water MASER emission in the atmosphere of Jupiter induced by a cometary impact. ${ }^{1}$ Our measurements have shown that the water MASER line at $22 \mathrm{GHz}$ can be used as a powerful diagnostic tool for planetary search outside the Solar System, as comets are able to deliver huge amounts of water (about 50 billion tons per comet) in planetary atmospheres raising the probability of LIFE evolution. Moreover calculations have shown that this line can be observed also in water-rich atmospheres where the necessary MASER pumping can be delivered by nearby IR-stars. By using a new developed fast multichannel spectrometer (132.000 channels) in a bandwidth up to $8 \mathrm{MHz}$, coupled to the 32 meters dish of the Medicina Radiotelescope, we started in 1998 the search for water in seventeen different targets up to 50 light years of distance from the Sun. The chosen targets are stellar regions where cometary clouds have been discovered or where planetary systems have been indirectly detected (up to now seventy-seven exoplanets discovered) or peculiar stars, like red and brown dwarfs with strong IR-radiation. In this paper we will illustrate the results achieved up to now, which show reliable water emission in planetary systems around Epsilon Eridani, Upsilon Andromedae and Lalande 21185. If confirmed this would be the first discovery of water in exo-planetary atmospheres showing the possibility of past, present or future life development.

Reference

${ }^{1}$ Cosmovici et al., Planet. Space. Sci., 44, 735 (1996).

ESA's search for Extra-solar terrestrial planets: mission up-date of the DARWIN project

L. Kaltenegger

Scientific Project Department, ESA/ESTEC, Postbus 299,

2200 AG Noordwijk, The Netherlands, also, at Institute of Geophysics, Astrophysics and Meteorology, University of Graz, Austria,

E-mail: lisa.kaltenegger@esa.int
A mission status is given for the Darwin project. The Infra Red Space Interferometer Darwin was studied, by the European Space Agency (ESA), at system level, between 1997 and 2000. The study, carried out by Alcatel space division in Cannes, France has focussed on developing a system that could carry out two main scientific objectives: (1) The detection and characterization of Earth-like planets orbiting other stars. (2) The imaging of astrophysical objects with unprecedented spatial resolution. The most challenging of these objectives consist of the recording of infrared spectra of Terrestrial exo-planets that could detect signs of biological activity at distances up to $20 \mathrm{pc}$. In order to do this, the Darwin project is constructed around the new technique of 'nulling interferometry', which exploits the wave nature of light in order to extinguish the light from a bright object (the central star, the primary, in this case). At the same time the light from a nearby source (the planet) is enhanced. The contrast between planets and stars being the least in the infrared wavelength region, that has been chosen for this mission.

\footnotetext{
The Exo/Astrobiology input of a Darwin/TPF mission F. Selsis

Centro de Astrobiología (CSIC-INTA), Ctra. de Ajalvir, $\mathrm{km} \mathrm{4,}$ Torrejón de Ardoz, 28850, Madrid, Spain,E-mail: selsis@observ.ubordeaux.fr

Beyond the highly exciting and debated search for spectroscopic biosignatures with the future space observatories under study by ESA (Darwin) and NASA (TPF-Terrestrial Planet Finder), the capability of producing low-resolution spectra of terrestrial exoplanets will be a major source of new information for planetary science and astrobiology. By expanding the known sample of terrestrial planets and atmospheres, a mission of this type will give a new dimension to these fields of research. The atmospheric composition deduced from the planetary thermal spectra, when compared to the specific characteristics of the observed systems - age, planetary distribution of terrestrial and giant planets in distance and size, stellar type, metallicity - will provide new views and constraints on the following topics: (1) origins of water and volatile species, their link with the existence and distibution of giant planets; (2) evolution of planetary atmospheres; (3) composition of prebiotic atmospheres (in the case of very young systems); (4) circumstellar habitable zone - observation of the $\mathrm{CO}_{2}$ level, and of other greenhouse gases.
}

\section{Scientific Objectives for Future Mars and Planetary Exploration}

\author{
Statement on planetary protection in Europe and \\ upcoming works \\ A. Debus \\ Centre National d'Etudes Spatiales, CNES, 18 Avenue Edouard Belin
}

31401 Toulouse, Cedex 4, France,E-mail: andre.debus@cnes.fr

Since the beginning of the last decade, ESA and CNES have been involved in Solar System exploration missions where Planetary Protection requirements have to be applied, an area where NASA has been involved since the beginning of the sixties. Main works have been accomplished by US scientists and in COSPAR in order to build and to propose an international policy and specifications. European scientists have now to be involved in this topic. The presentation will give the statement of Planetary Protection in Europa, and particularly at CNES, in order to begin a reflexion and to determine activities that could be undertaken at the European level. The following topics will be presented: Organization (Planetary Protection committee, responsibilities inside Agencies, reflexion of an European Planetary Protection policy, European Planetary Protection standard using CNES one). Forward contamination prevention: new statements and propositions to COSPAR (Mars and Europa exploration particularly). Back contamination prevention.
Risks determination, quarantine analysis protocols. Sample preservation for exobiology purpose: needed research and development activities.

\section{Scientific objectives for Mars for several decades -} the compilation of scientific objectives for Mars exploration and a time schedule for the next decades V. Mang Space Infrastructure Division, Astrium GmbH, PO Box 286156 , 28361 Bremen, Germany, E-mail: volker.mang@astrium-space.com The scientific objectives for Mars exploration, from the Astrobiology point-of-view, can be categorized into three groups: (1) the origin of life: former and actual conditions on Mars influencing life's origin and survival, extent and extinct life; (2) the limits of Earth life: native and engineered organisms in Mars and interplanetary flight environmental conditions; (3) engineering tasks and applied sciences for the optimization of robotic and human exploration. The goals and objectives with relation to human presence on Mars are an integral part of each of the groups. The nature of the scientific objectives covers the interests of life scientists as well as planetologists and others. Very often, it is possible to 
investigate these objectives and goals not only on Mars but also on Earth. Sometimes it is not even necessary to perform the experiments on Mars, a lot of them can be performed on Earth. The investigations on or about Mars facts are valid not only for Mars; they may also have close relations to research activities concerning Earth facts. The study gives the objectives and goals within their structure. Some examples for the relation to science on Earth and with interfaces to other objectives and goals will be discussed. An attempt has been made to identify a broad list of research disciplines (nearly eighty) to find tasks that the scientists from this special discipline could perform as a contribution to this interdisciplinary research field. Some examples will be described. Finally, a sketch of a relative schedule of the research themes will be discussed.

\section{Life Detection Methods on Mars}

\section{Miniaturization concepts for a space-born Raman spectroscopy instrument}

S. Hofer(1), E. Schmidt(1), T. Stuffler(1), J. Popp(2), N. Tarcea(2), M. Schmitt(3), W. Kiefer(3)

(1) Kayser-Threde GmbH, Wolfratshauser Str. 48, D-81379 München, Germany, E-mail:sh@kayser-threde.de; (2) Institut für Physikalische Chemie, Universität Würzburg, Am Hubland, D-97074 Würzburg, Germany; (3) Institut für Physikalische Chemie, Friedrich-SchillerUniversität Jena, Helmholtzweg 4, D-07743 Jena, Germany

In the last few years Raman spectroscopy has been identified as an advanced method for in-situ planetary analysis. The most important fields that Raman spectroscopy is addressing are the mineralogical and organic/biological analyses on micrometer size scale. Thinking about the realization of a Raman instrument for planetary observations, the driving design constraints are the limited resources on those missions like power, mass and instrument envelope. From the measurement point of view the main design driving constraint is caused by the weakness of the Raman signal itself, which is in the order of $10^{-8}$ referred to the stimulating light input. In an ongoing breadboard study, two different approaches are followed to set up a space born Raman instrument. The two presented instrument concepts differ mainly in the spectrometer part. The first is based on an AOTF (Acusto-Optic Tunable Filter) in combination with an APD detector, the second approach uses an improved Hadamard spectrometer with CCD detector. Measurements with the breadboard are used to collect specific instrument data for further concept improvements. Preliminary assessments show that a Raman instrument with a total volume of $150 \times 150 \times 150 \mathrm{~mm}^{3}$, a total mass of less than $2 \mathrm{~kg}$ and a total power consumption of less than $10 \mathrm{~W}$ is feasible. In the paper, first results from the breadboard evaluation tests and an outlook with respect to a suitable space-born instrument configuration will be presented.

\section{Imaging of terrestrial Mars analogue materials using the Beagle - 2 camera system}

B. A. Hofmann(1), M. Josset(1), J.-L. Josset(2)

(1) Natural History Museum Bern, Bernastrasse 15, CH-3005, Bern, Switzerland,E-mail: beda.hofmann@nmbe.unibe.ch,

(2) Micro-Cameras \& Space Exploration (SPACE-X), Jaquet-Droz 1, CH-2007 Neuchâtel, Switzerland

The reconstruction of the geological environment close to future Mars landing sites, particularly of Beagle - 2, the lander of ESA's Mars Express mission, will heavily depend on the interpretation of optical images. Besides reconstructing the geological environment, close-up images of rocks will be used in the search for possible biogenic textures (e.g. stromatolites, filamentous fabrics). We have started a study of terrestrial rocks and meteorites using a camera system identical to that on board Beagle - 2, the lander of ESA's Mars Express mission. Terrestrial materials imaged include a wide variety of potential Mars analogue materials such as detrital and chemical sediments (playa sediments, sandstone, conglomerate, limestone), igneous and volcanic rocks (gabbro, basalts, andesites), several types of impact-produced rocks (breccia, impact melt). Meteorites include several Mars meteorites (including the main mass of Sayh al Uhaymir 094) and typical ordinary and carbonaceous chondrites. Besides normal rocks, several types of verified or suspected biogenic structures are being imaged: Stromatolites and a range of examples of filamentous fabrics from subsurface environments. Both naturally weathered and freshly broken surfaces are imaged. Images are taken as stereo pairs at a distance of $60 \mathrm{~cm}$ with a resolution of approx. $0.4 \mathrm{~mm} /$ pixel (at the image center) and, additionally, with a close-up lens (that will be incorporated in the filter wheel, allowing blackand white images only) at $8 \mathrm{~cm}$ distance with a resolution approaching $50 \mathrm{micron} /$ pixel. Characteristic textures of rocks and of fossil microbial fabrics are well discernible in these images, especially when using the close-up lens, confirming that optical imaging using Beagle-2-type instrumentation will allow a much more detailed interpretation of the petrology of rocks than was possible during earlier missions. A routine allowing a standardized systematic interpretation of the images is being developed. Additionally, we plan blind interpretation tests using images taken of unknown materials. This study is part of the characterization of Mars reference materials using instruments on Beagle -2 .

\section{Some promising approaches to analysis of the physiological state of microorganisms in situ} A. L. Mulyukin, N. E. Suzina, V. V. Sorokin, V. S. Soina, E. A. Vorobeva, V. V. Dmitriev, V. I. Duda, G. I. El-Registan Institute of Microbiology, Russian Academy of Sciences, INMI RAS, Pr. 60-letiya Oktyabrya 7/2, Moscow 117312, Russia, E-mail: andylm@rambler.ru

While it is commonly accepted that microorganisms in the environment exist in the dormant state, in which the cells can survive for prolonged periods under unfavourable conditions related to nutrient exhaustion or unavailability, deficiency of water, action of non-optimal temperatures and other physiochemical factors. Dormant cells, which are widespread in most of Earth habitats and can be regarded as a target to search for life or its evidence in extraterrestrial systems, were shown to differ significantly by morphology, maintenance of viability, resistance to extreme conditions, and residual metabolic activity. As it is well known in practices of microbiological monitoring, only a very small fraction of the microorganisms present in natural habitats can be enumerated by standard methods of plating on to nutrient media. Besides underestimation of the real total number of microorganisms, these indirect methods provide no information on physiological state of single microbial cells in situ. Therefore, searching for criteria to distinguish between microbial cells of different physiological states (including dormant forms) by direct methods is a very important task. To find such criteria, we compared the microbial cells, which were obtained under experimentally selected conditions and differed by their physiological state and growth potentialities in a row: actively growing and stationaryphase cells - dormant forms - non-viable micromummies - dead cells. Thus, comparative electron microscopy studies allowed us to reveal the ultrastructural peculiarities of dormant and mummified microbial cells, which can be used as their 'portrait' characteristics in analysis of microorganisms in situ. It is interesting that bacterial cells isolated immediately from permafrost sediments by low-temperature fractionation were very similar in their fine structure to dormant cystlike cells obtained in long-stored cultures, whereas yeast micromummies resembled the fossilized cell remnants in kerites. Besides this, there were statistically significant differences in the quantitative parameters (the content of some biologically important elements and their ratios) obtained by X-ray microanalytical studies of single microbial cells. Thus, the distinctive feature of all the studied dormant microbial cells was their low $\mathrm{P} / \mathrm{S}$ ratios and high $\mathrm{Ca} / \mathrm{K}$ ratio in contrast to growing and stationary cells, whereas non-viable micromummies, being morphologically intact, showed an anomalous content of potassium. Based on the data obtained for model microbial cells of different physiological states as the reference, we used SEM-XRMA to develop approaches to detect microorganisms 
among cell-like particles in samples of ancient permafrost sediments and of room dust. The absence of $\mathrm{P}$ and $\mathrm{S}$ peaks in $\mathrm{X}$-ray spectra recorded for the most part of cell-like particles allowed us to regard them as abiogenic objects. Among other particles that were recognized as more probable microorganisms, we suggest the presence of microbial cells with the content and ratios of elements similar to dormant cells. Hence, further studies of the resting state of microorganisms and revealing the characteristics specific for dormant cells is very promising for microbiological monitoring of terrestrial and extraterrestrial habitats.

\section{Sampling of palaeo-water from fluid inclusions in surface deposits on Mars}

J. Parnell, M. Baron, A. Wilkins, and the Geofluids Research Group University of Aberdeen, Department of Geology and Petroleum Geology, Aberdeen AB24 3UE, UK, E-mail: j.parnell@abdn.ac.uk The most viable deposits in which to search for evidence of life are those that were precipitated at or just beneath the planetary surface, where conditions suitable for life (by terrestrial analogy) probably pertained in the past. Most types of terrestrial surface deposit have been suggested as targets for the search for life on Mars. The targets identified include lake deposits in general and evaporites in particular, algal deposits, (stromatolites), cave deposits (speleothems), travertines, 'hot' spring deposits, 'cold' seep (chemosynthetic) deposits and duricrusts. The deposits of all of these environments contain water in the form of fluid inclusions and the water survives within samples in the terrestrial geological record. Therefore, if deposits of any one of them could be accessed on/from Mars, they should contain micro-samples of Martian water, susceptible to a range of analyses including the high-resolution detection of biomolecules. On Earth, encouragement stems from the very long-term survivability of biomolecules in some cases, preservation of biomolecules within hydrothermal sinters in particular, the possible preservation of microbes within evaporites in the geological record, the nucleation of many carbonate crusts upon biological templates and the preservation of organic breakdown products within diagenetic arbonate crusts upon biological templates and the preservation of organic breakdown products within diagenetic mineral precipitates. Samples from all of the target deposit types have been selected from the geological record, in which the predominant mineral phase, usually calcite or aragonite, is sufficiently coarsely crystalline to allow detection of fluid inclusions. In every type of deposit we have identified fluid inclusions that we conclude represent early, depositional or shortly postdepositional fluids. There are several reasons to believe that this conclusion is justified: (1) They are consistently monophase inclusions, characteristic of inclusions trapped at temperatures less than about $60{ }^{\circ} \mathrm{C}$. (2) In some cases, later fluid populations are recorded in the same samples as the early fluids, but have not displaced the early fluids from their inclusions. (3) Petrographic studies of the host carbonates indicate that they are primary, not recrystallized, fabrics. Some samples from the geological record only yield an inclusion record representative of deep burial. This is an inevitable consequence of diagenetic processes. However, in the context of planetary exploration, obtaining any fluid record would be invaluable, and it is encouraging that so many samples of surface precipitate have yielded such a record. A brief summary of the case for the occurrence of such deposits on Mars is given below. Evaporites: an extensive literature on prospective evaporite deposits on Mars, focussed on palaeolacustrine environments, suggests that site selection should include these deposits. Evidence for evaporites includes Martian albedo data and their occurrence in meteorites of Martian origin. The occurrence of halophilic bacteria on Earth makes evaporites an attractive target in the search for life on Mars. Duricrusts: evidence for cemented soil (duricrust) at the Viking lander sites, combined with geochemical data, suggests that the surface may contain sulphate-rich precipitates, most likely epsomite or gypsum, precipitated from concentrated brines. Terrestrial duricrusts particularly involve calcrete (caliche), and similar carbonate crusts could develop on basalt surfaces on Mars. Biogenic lacustrine precipitates: Paleolake deposits in general are advocated as an astrobiological target. By analogy with the terrestrial record of life, simple microbial populations may have precipitated a mineral record with a distinctive and recognisable morphological character, like stromatolites. These precipitates would most likely be a feature of standing bodies of water or fluid seepage zones. Hydrothermal precipitates: a particular type of fluid seepage is the hot spring, which is an attractive target on Mars as it represents a means of introducing liquid water to the planetary surface, primitive life could be thermophilic, and a record of life could be preserved in mineral precipitates from the cooling waters. Chemosynthetic precipitates: 'cold' seepages represent another type of seepage that may reach the surface, but unrelated to hydrothermal activity, and are also a prospective environment for primitive life. Chemosynthetic communities do not need photosynthesis or oxygen, drawing energy directly from compounds like methane that emerge at cold seepage sites. Terrestrial chemosynthetic communities precipitate distinctive carbonate crusts, which are also proposed as targets on Mars, where there is some morphological evidence for cold outgassing. Speleothems: a wide diversity of primitive life colonizes subsurface void spaces ('caves'), a potential environment that offers protection from harmful radiation on the surface. Water in these spaces precipitates crusts (speleothems), which may entrap the ambient life, and which are therefore a plausible environment for exploration on Mars. Our demonstration that each of these deposit types contains included water shows that there is an excellent chance of accessing Martian palaeo-water samples in surface precipitates. A first stage in sampling would involve planetary spectroscopic techniques capable of detecting hydrothermal deposits, carbonates and evaporites. Of the various types of deposit discussed, hydrothermal deposits and evaporites may be the most widespread on Mars. Upon liberation of the inclusion fluids from samples, they could be analysed using a number of approaches advocated for the sensitive determination of organic compounds on Mars, including gas chromatography-mass spectrometry, liquid chromatography, immunoassay or polymeric sensors.

\section{Spectroscopic search for signatures of fossils in terrestrial layered rocks}

R. Politi, G. A. Marzo, A. Blanco, F. De Carlo, S. Fonti, A. C. Marra, $V$. Orofino

Physics Department, University of Lecce, C.P. 193, 73100 Lecce,

Italy, E-mail: romolo.politi@le.infn.it

Many observations of present Mars suggest that there was liquid water on its surface for a time probably long enough to be compatible with the development of some very simple forms of life. Moreover other evidences, such as the recently discovered gullies, indicate that the epoch in which liquid water was present is not so far in the past. For this reason the interest about the possibility of discovering the signs of an ancient biological activity on Mars is growing, as underlined by the very lively debate arisen around the claimed discovery of fossils bacteria in the Martian meteorite ALH84001. Experiments to check the presence of some form of life on the surface of Mars have been already attempted by the first landers in the late seventies without reaching definitive answers. Similar techniques will certainly be used in future landing experiments, but starting with the Mini-TES spectrometer on board of the Athena Rover planned for the 2003 MER mission, spectroscopic investigations will also be possible. One of the advantages of such techniques is that in principle it would be possible to detect also the presence of fossilized forms of life. However, in order to understand the data that will be supplied by Mini-TES and other similar instruments, a detailed laboratory study of carefully chosen terrestrial samples is necessary. For this purpose we have started a systematic analysis of the differences between two materials, identical in composition but with different fossil content, using the properties of a particular geological stratigraphic structure, the K-T (Cretaceous - Triassic) sequence. The geologic separation between the $\mathrm{K}$ layer, rich in fossils, and the $\mathrm{T}$ one, poor in fossils, is marked by a thin Iridium-enriched layer which suggests a catastrophic impact undergone by Earth at the end of Cretaceous. This is supposed to be the cause of a major mass extinction event. In those sites in which it is possible to recognize the Iridium-enriched layer, the layers immediately above and below represent respectively ages with weak and strong biological activity. Such a place is located near Gubbio (Italy) where the 
$\mathrm{K}-\mathrm{T}$ sequence is included in a sedimentary deposit with a homogeneous composition during the entire sequence. Selected rock samples carefully chosen in the proximity of the Iridium-enriched layer have been delivered to our laboratory for appropriate analysis. In this paper we report on the protocol we plan to use for the above mentioned rocks in order to identify the possible spectroscopic differences linked to the presence of fossils. The samples will be examined both as slabs and as particulate material. A thorough characterization will be performed using SEM and EDX analysis and the size of the particulate will be selected using sieving and sedimentation techniques. The spectroscopic analysis will include transmittance, reflectance and emission spectra taken in the widest possible range from the near UV to the far IR. The complete analysis will require several months but we plan to have preliminary results in the next six to eight weeks. Even if such preliminary results might not be conclusive we trust that they could nevertheless give some interesting hints important for the future understanding of evolution of life on Mars.

\section{Raman spectroscopy as a suitable tool for biological and mineralogical in-situ planetary studies \\ J. Popp (1,2), N. Tarcea(1), M. Schmitt(1), W. Kiefer(1), \\ R. Hochleitner(3), G. Simon(3), M. Hilchenbach(4), S. Hofer(5), T. Stuffler(5)}

(1) Institut für Physikalische Chemie, Universität Würzburg, Am Hubland, D-97074 Würzburg, Germany; (2) Institut für Physikalische Chemie, Friedrich-Schiller-Universität Jena, Helmholtzweg 4, D-07743 Jena, Germany, E-mail: juergen.popp@uni-jena.de; (3) Mineralogische Staatssammlung Müchen, Museum Reich der Kristalle, Theresienstr. 41, D-80333 München, Germany; (4) Max-Planck-Institut für Aeronomie, Max-Planck-Str. 2, D-37191 Katlenburg-Lindau, Germany; (5) Kayser-Threde GmbH, Wolfratshauser Str. 48, D-81379 Munich, Germany

In the last few years Raman spectroscopy has been identified as a possible method for in situ planetary analysis. The most important fields that Raman spectroscopy is addressing are the mineralogical and organic/ biological analysis at the micrometer size scale. The advantages and the limitations of Raman spectroscopy as a method of investigation for future planetary missions are discussed here, and, as an illustration, this method has been used for analysing samples of known origin. Fossil-bearing rocks as well as water containing minerals were analysed. Raman spectroscopic imaging of microscopic fossils and point measurements on macroscopic fossils were used as means of identifying the fossils and to substantiate their biological origin. Raman imaging when used in correlation with the optically discernible morphology of the sample proved to be very effective in establishing the biogenicity of the investigated sample. Fossilized fungi and hematite in quartz, from Warstein, Germany, fossilized coral (Triassic age) from Bad Aussee, Austria, and fossilized mussel shell (Tertiary age) from Harz, Germany, were used for measurements. Also a recent seaurchin fossil, and a petrified wood sample from Queensland, Australia (inv. no. 17313*) were measured employing Raman spectroscopy. Kaolinite, $\mathrm{Al}_{4} \mathrm{Si}_{4} \mathrm{O}_{10}(\mathrm{OH})_{8}$, Sumatra (Indonesia), (inv. no. 12799*) and Montimorillonite, $(\mathrm{Al}, \mathrm{Mg})_{2} \mathrm{Si}_{4} \mathrm{O}_{10}(\mathrm{OH})_{2}(\mathrm{Na}, \mathrm{Ca})_{\mathrm{x}} * 6 \mathrm{H}_{2} \mathrm{O}$, from Fuchsbau (inv. no. 9237*) are examples of clay minerals used for illustrating the possibility of detecting water by means of Raman spectroscopy. Furthermore, concepts of a miniaturized Raman apparatus will be presented.

* The samples were provided by, and are under the custody of, the Mineralogical State Collection in Munich, Germany.

\section{Calcified biofilm architecture: mineralized evidence of ancient microbial life \\ R. Riding}

Earth Science Department, Cardiff University, Cardiff CF10 3 YE,

UK, E-mail: riding@cardiff.ac.uk

Recent work has questioned both chemical evidence of life $~ 3800$ million years $\operatorname{ago}^{1}$ based on carbon-isotope values in the Isua Group of southern Greenland, ${ }^{2,3}$ and bacteria-like filaments ${ }^{4}$ in the $\sim 3450$ million year Towers Formation of the Warrawoona Group of Western Australia. ${ }^{5}$ Doubts have been raised about the biogenicity of Warrawoona and other stromatolite-like structures older than 3200 million years ${ }^{6}$ and the nature of nanobacteria-like objects on Earth ${ }^{7}$ and Mars. ${ }^{8,9}$ In view of this uncertainty regarding chemical, fossil, microfabric and bacteriform evidence, how can the search for life in old rocks on Earth, and in space, be advanced? If we cannot rely on these putative signs of life, are there others that may be worth exploring? Microbial communities in aquatic environments colonize surfaces by forming adherent biofilms. Intuitively, these might be expected to have poor preservation potential and/or to be insufficiently distinctive for confident recognition. However, there are calcified examples up to 500 million years old from a variety of cryptic habitats in shallow marine sediments that preserve architectural features typical of modern unmineralized biofilm. ${ }^{10} \mathrm{In}$ comparison to typical stromatolite microstructure, biofilm architecture possesses more detail, and in comparison to cell and filament appearances may be less readily mimicked by abiotic processes. Biofilms are adherent bacterial populations in a matrix of extracellular polymeric substances (EPS) and are extremely widespread in modern aquatic environments. ${ }^{11}$ They are thin organic layers, typically tens to a few hundreds of microns in thickness. The EPS matrix acts as a diffusion barrier and adsorbent and allows microbes consortial opportunities for metabolic interaction in a stable microenvironment. ${ }^{12,13,11}$ Biofilms preserved by mineral precipitation during their formation could have an extraordinarily long fossil record. Thin $(<150 \mu \mathrm{m})$ micritic veneers lining crypts in reef, microbial, and bioclastic limestones up to 510 million years old possess the dimensions and architecture of modern uncalcified bacterial biofilm. Morphologic attributes include rounded aggregate nanofabric, internal channels, external towers, mushrooms, and plumes. All can be interpreted as characteristics of attached bacterial communities, i.e., aggregates as microcolonies, originally embedded in a matrix of extracellular polymeric substances; channels as water conduits and/or uncolonized nutrient-poor spaces; external protuberances as localized growths; and plumes as surface streamers. Cryptic habitat favoured pristine biofilm preservation by precluding disturbance and overgrowth, and suggests aphotic and anoxic conditions. These examples provide diagnostic morphologic criteria for wider recognition of biofilm in ancient carbonate rocks. Little has been known until recently about ancient marine calcified biofilm. Studies of microbial carbonates have instead largely concentrated on the diverse sedimentary products of microbial mats that include stromatolites and thrombolites. Classic biofilms are much thinner than mats, and typically are built by heterotrophic cells with dimensions less than $2 \mu \mathrm{m}$. The resulting architectural features are quite small. Furthermore, EPS calcification that can preserve bacterial deposits typically results in fine-grained micritic fabrics ${ }^{14}$ that are difficult to decipher. ${ }^{15}$ Consequently, the quality of preservation of distinctive delicate biofilm features in these examples is unexpected. Their recognition is a significant step that allows the anticipated importance of ancient bacterial carbonates to be explored utilizing specific morphologic criteria for biofilm identification. These biofilm fossils confront the notion of ubiquitous preservation of isolated bacteria and nanobacteria and at the same time show that distinctive biofilm microfabrics are preservable in examples up to $\sim 500$ million years old. There is every likelihood that much older examples will be recognized.

References

${ }^{1}$ Fedo, C. M. and Whitehouse, M. J. (2002). Metasomatic origin of quartz-pyroxene rock, Akilia, Greenland, and implications for Earth's earliest life. Science 296: 1448-1452.

${ }^{2}$ Schidlowski, M. (1988). A 3,800-million-year isotopic record of life from carbon in sedimentary rocks. Nature 333: 313-318.

${ }^{3}$ Mojzsis, S. J., Arrhenius, G., McKeegan, K. D., Harrison, T. M., Nutman, A. P. and Friend, C. R. L. (1996). Evidence for life on Earth before 3,800 million years ago. Nature 384: 55-59.

${ }^{4}$ Brasier, M. D. et al. (2002). Questioning the evidence for Earth's oldest fossils. Nature 416: 76-81. 
${ }^{5}$ Awramik, S. M., Schopf, J. W. and Walter, M. R. (1983). Filamentous fossil bacteria from the Archean of Western Australia. Precambrian Research 20: 357-374.

${ }^{6}$ Lowe, D. R. (1994). Abiological origin of described stromatolites older than 3.2 Ga. Geology 22: 387-390.

${ }^{7}$ Folk, R. L. (1993). SEM imaging of bacteria and nannobacteria in carbonate sediments and rocks. Journal of Sedimentary Petrology 63: 990-1000.

${ }^{8}$ McKay, D. S., Gibson, E. K. Jr., Thomas-Keprta, K. L., Vali, H., Romanek, C. S., Clemett, S. J., Chillier, X. D. F., Maechling, C. R. and Zare, R. N. Search for past life on Mars; possible relic biogenic activity in Martian meteorite ALH84001. Science 273: 924-93.

9 Vali, H. et al. (2001). Nanoforms: a new type of protein-associated mineralization. Geochimica et Cosmochimica Acta 65: 63-74.

${ }^{10}$ Riding, R. (2002). Biofilm architecture of Phanerozoic cryptic carbonate marine veneers. Geology 30: 31-34.
${ }^{11}$ Costerton, J. W., Lewandowski, Z., Caldwell, D. E., Korber, D. R., and Lappin-Scott, H. M. (1995). Microbial biofilms. Annual Review of Microbiology 49: 711-745.

${ }^{12}$ Lawrence, J. R., Wolfaardt, G. M. and Korber, D. R. (1994). Determination of diffusion coefficients in biofilms by confocal laser microscopy. Applied Environmental Microbiology 60: 1166-1173.

${ }^{13}$ Wolfaardt, G. M., Lawrence, J. R., Robarts, R. D., Caldwell, S. J., and Caldwell, D. E. (1994). Multicellular organization in a degradative biofilm community. Applied Environmental Microbiology 60: 434-446.

${ }^{14}$ Riding, R. (2000). Microbial carbonates: the geological record of calcified bacterial-algal mats and biofilms. Sedimentology 47 (Supplement 1): 179-214.

${ }^{15}$ Reid, R. P., Macintyre, I. G., and James, N. P. (1990). Internal precipitation of microcrystalline carbonate: A fundamental problem for sedimentologists. Sedimentary Geology 68: 163-170.

\section{The Search for Extra-Solar Planets, Biosignatures and Habitable Zones}

\section{The habitable zone of Earth-like planets around $47 \mathrm{UMa}$ \\ W. von Bloh \\ Potsdam Institute for Climate Impact Research, PO Box 6012 03, \\ 14412 Potsdam, Germany, E-mail:bloh@pik-potsdam.de}

The system of 47 UMa consists of two Jupiter-size planets beyond the outer edge of the stellar habitable zone, and thus resembles our own solar system most closely compared to all extrasolar planetary systems discovered so far. The habitability of this system for Earth-like planets is investigated following a concept, which assumes the long-term possibility of photosynthetic biomass production under geodynamic conditions. Consequently, a broad variety of climatological, biogeochemical, and geodynamical processes involved in the generation of photosynthesis-driven life conditions is taken into account. In particular, the stellar luminosity and the age of star/planet system are of fundamental importance for planetary habitability. Our study considers different types of planetary continental growth models and takes into account a careful assessment of the stellar parameters. In the event of successful formation, we find that Earth-like habitable planets around $47 \mathrm{UMa}$ are in principle possible. The likelihood of those planets is increased if it assumed that $47 \mathrm{UMa}$ is relatively young and has a relatively small stellar luminosity as permitted by the observational range of those parameters.

\section{Hierarchical structuring of planetary systems in the framework of scale relativity \\ P. H. M. Galopeau(1), L. Nottale(2), D. Da Rocha(2), \\ G. Schumacher(3), N. Tran Minh(4) \\ (1) CETP, CNRS, Vélizy, France, E-mail: \\ patrick.galopeau@cetp.ipsl.fr; (2) LUTH,CNRS, \\ Observatoire de Paris-Meudon, France; (3) Observatoire de la Côte \\ d'Azur, Nice, France; (4) LERMA, CNRS, Observatoire de \\ Paris-Meudon, France}

For several years, many planetary systems have been discovered around nearby stars. The distance between these planets and the central star is a key parameter for the appearance of life. Other parameters also play a basic role: the mass, the obliquity, the magnetic field. Although these planetary systems may look very different from our solar system, they actually present universal structures comparable to ours; the same remark can be made about the satellite systems of the giant planets. The existence of such structures in planetary systems, organized in a hierarchical way, is suggested by the theory of scale relativity. In the framework of the scale relativity approach, the motion at relatively large time-scales of the bodies in the solar system is described by fractal trajectories, governed by a Schrödinger-like equation. Preferential orbits are predicted in terms of probability density peaks with semi-major axis given by: $a_{n}=G M n^{2} / w^{2}$ (M is the mass of the Sun and $w$ is a velocity close to $144 \mathrm{~km} \backslash \mathrm{s}^{-1}$ for the inner solar system). The velocity of the planet orbiting at this distance satisfies the relation $\mathrm{v}_{\mathrm{n}}=\mathrm{w} / \mathrm{n}$. Moreover, the mass distribution of the planets can be accounted for in this model. These predictions are in agreement with the observed values of the actual orbital parameters. Furthermore, the exoplanets which have been recently discovered around nearby stars also follow the same law in terms of the same constant in a highly significant statistical way. The theory of scale relativity also predicts structures for the obliquities and inclinations of the planets and satellites: the probability density of their distribution between 0 and $\pi$ are expected to display peaks at particular angles $\theta_{\mathrm{K}}=\mathrm{K} \pi / \mathrm{n}$. A statistical agreement is obtained for our solar system with $\mathrm{n}=7$. Another prediction concerns the distribution of the planets eccentricities $\mathrm{e}$. The theory foresees a quantization law $\mathrm{e}=\mathrm{k} / \mathrm{n}$ where $\mathrm{k}$ is an integer and $\mathrm{n}$ is the quantum number that characterizes semi-major axes. The presently known exoplanet eccentricities are compatible with this theoretical prediction. An interesting consequence of our study is that one can expect with a high probability to discover exoplanets having orbital characteristics very similar to the Earth's ones.

\section{The role of biospheres in the formation of planetary climate: Greenhouse catastrophe}

\section{A. V. Karnaukhov}

Institute of Cell Biophysics, Russian Academy of Sciences, Puschino 142290 Moscow reg., Russia, E-mail: akarn@icb.psn.ru

The role of processes in organic and inorganic nature that form chemical composition of the atmosphere and temperature balance was analysed. It was found that the biological mechanisms of $\mathrm{CO}_{2}$ removal from the atmosphere are insufficient, and there exist potentially hazardous $\mathrm{CO}_{2}$ sources in inorganic nature that can be activated with the increase of the global Earth temperature. It was shown that the stability of natural systems of maintaining the constant chemical composition of the atmosphere is disturbed due to the destruction by man of climate-forming biocenoses, which may lead to irreversible changes in Earth's climate that may result in increased planetary temperature up to $100-150{ }^{\circ} \mathrm{C}$ and higher. This would make the existence of life on our planet or on a terrestrial planet where similar processes may occur or may have occurred impossible; also orbits around the star in a habitable zone. A radiation-adiabatic model of the greenhouse effect was created, in which a framework of asymptotic estimations of the increase in the global planetary temperature for the wide range changes of the concentration of greenhouse gases were obtained. On the basis of this model, an integral model of changes in Earth's climate was created, which takes into account the heat inertia of the ocean and aerosol contaminations of the upper layers of the atmosphere. It is shown that irreversible (catastrophic) changes in Earth's climate (greenhouse catastrophe) may occur in a relatively near future (200-300 years). The presented climate model 
demonstrates perfect accordance with various observational data, for instance: instrumental measuring of the Earth temperature in the twentieth century, greenhouse effect on Venus, various paleoenvironmental records, including the results of deep-hole drilling in the Antarctic glacier in the Russian Vostok station.

\section{Planets and planetary systems: a contrarian viewpoint \\ S. S. Kumar}

The Galileo Institute and University of Virginia, P.O. Box 6516

Charlottesville,VA22906USA,E-mail: galileo@cstone.net

Arguments are presented to show that planetary systems, in general, are not formed as byproducts of the star formation processes. Consequently, the occurrence of stable planetary systems is unlikely to be a universal phenomenon in the Galaxy. Comments are made on the origin and evolution of life on the Earth, and it is suggested that life-as-we-know-it on the Earth is a local phenomenon and that the existence of such life is likely to be quite rare in the Galaxy and beyond.

\section{The detectability of terrestrial atmospheres by space transit spectroscopy \\ A. J. Penny, K. M. Smith}

Rutherford Appleton Laboratory, Chilton Didcot OX110QXUK,

E-mail: alan.penny@rl.ac.uk

The use of transmission spectroscopy for detecting and investigating the atmosphere of a terrestrial planet as it transits in front of its parent star is investigated. The performance of a telescope in space under different assumptions about the planet atmospheres is calculated.

\section{Radioastronomical aspects in the search for extra solar planets \\ H. O. Rucker}

Department for Extraterrestrial Physics, Space Research Institute, Austrian Academy of Sciences, Schmiedlstr. 6, A-8042 Graz, Austria, E-mail:helmut.rucker@oeaw.ac.at
In analogy to our solar system in which Jupiter with a dominating planetary magnetic field acts as a powerful non-thermal radio emitter, it is reasonable that giant exoplanets exhibit comparable radiation in the decametric wavelength regime. State-of-the-art instrumentation incorporated in huge ground-based radio telescopes may be able to discover these electromagnetic signatures, maybe only by means of sophisticated observational methods. Some of these aspects are addressed in view of recent developments.

\section{Biosignatures and exoplanet characterization: visible versus infrared \\ J. Schneider}

Paris Observatory, CNRS, 5 place Jules Janssen 92195 Meudon,

France, E-mail: jean.schneider@obspm.fr

The full understanding of possible presence of life on exoplanets depends on the simultaneous combination of two factors: (1) the detection of biosignatures themselves and (2) the understanding of physical conditions (temperature, density of the atmosphere, surface conditions, if an ocean is present is it liquid or frozen?, day/night contrast, duration of the day etc.).With this in mind, I will discuss the case of biosignatures and exoplanet characterization for reflected stellar light (in the visible) versus thermal emission (in the infrared) by: (1) reviewing the classical arguments for both wavelength regions (planet radius, temperature, albedo), (2) discussing some artefacts (planetary rings, abiotic production of oxygen etc), (3) presenting new perspectives for both regimes (new methods for the detection of moons (in reflected light and thermal emission), atmosphere density, lithospheric and oceanic thermal heath transfer from infrared observations etc.). Some new aspects of the implementation of the planet characterization by ground-based programmes and space projects will be presented and discussed.

\section{Posters}

\section{NAI/UCLA Pathfinder Project}

C. Aguilar, L. Farrington, S. S. Mayerberger

UCLA 3806 Geology Building Department of Earth and Space Sciences UCLA, Los Angeles, CA 90095-1567,E-mail: thiag@ucla.edu; Marylou7@ucla.edu; sabrina@ess.ucla.edu

Due to increasing interest in the field of astrobiology, NASA's Astrobiology Institute (NAI) has recently been overwhelmed with emails requesting, essentially, advice on how to become an Astrobiologist. The Pathfinder project was created in order to answer these questions, with the goal of producing a tool, accessible via the World Wide Web that will allow students to look up the astrobiology-related courses and programmes offered at educational institutions in their respective areas. Pathfinder is comprised of a group of student researchers from the University of California at Los Angeles and is supported by NASA's Astrobiology Institute.

\section{Origin and evolution of the UCLA Astrobiology}

\section{Society}

C. Aguilar, L. Farrington, S. S. Mayerberger

3806 Geology Building Department of Earth and Space Sciences

UCLA, Los Angeles, CA 90025-1567,USA,E-mail: thiag@ucla.edu, Marylou7@ucla.edu; sabrina@ess.ucla.edu

Co-sponsored by the NASA Astrobiology Institute (NAI) at UCLA, and the Center for the Study of Evolution and the Origin of Life (CSEOL), the UCLA Astrobiology Society (ABS) was founded in 1999 to become the first student-run organization devoted to Astrobiology. An interdisciplinary group, ABS unifies undergraduates, graduate students, and faculties of all fields through events and discussions centred around the diversity of themes that compose Astrobiology science. ABS provides particular benefit to NAI's education and public outreach efforts as a means of increasing awareness of and appreciation for Astrobiology, particularly in the college community. A greater goal of the organization involves networking to seed Astrobiology Societies on other college campuses nationwide. Additionally, ABS is currently doing research for NAI's Astrobiology Pathfinder programme, which will help students at all stages to become astrobiologists.

\section{The survival of complex prebiotic interstellar molecules}

S. Aiello(1), C. Cecchi-Pestellini(2)

(1) Department of Astronomy and Space Science, University of

Firenze, Largo E. Fermi, 2, I-50125, Firenze, Italy, E-mail: aiello@unifi.it,santi.aiello@arcetri.astro.it; (2) Department of Astronomy and Space Science, University of Florence,

Largo E. Fermi 2, I-50125, Firenze, Italy

It is well known that comets and planetesimals hit the Earth in a massive way, until four billion years ago. These objects probably retained the prebiotic material present in the pre-solar nebula and deposited it on our planet. The chemistry of organic molecules and their voyage from molecular clouds to the early solar system has been extensively studied in the last few years through laboratory simulations and astronomical observations from ground and space. ${ }^{3}$ Still, the evolution, survival, transport and transformation of the interstellar organic material is far from well understood. In this work we will investigate the possibility for organic matter to survive in space and seed planetary systems with the prebiotic molecules needed for proteins and nucleic acids in living 
organisms. Our study is based on the work of one who noted that the chemistry within aggregated grains offers a completely new perspective with respect to one in action on grain surfaces. ${ }^{2}$ In fact, chemical compounds different from those manufactured in the gas-phase or on the grain surface are to be expected. The re-accretion of reaction products by other components of the aggregate creates an environment where secondary reactions are facilitated. In addition, the passage of a cosmic-ray through the aggregate produces a transient desorption of volatile species which cannot escape the aggregate. This gives rise to a peculiar situation where chemistry is offered high temperature and high density conditions in the gas-phase, with rapid quenching of the reaction products. The effective production of complex molecules depends on the dose of UV radiation impinging on the molecular material trapped within aggregate grain sub-units. We will treat the penetration of UV light inside an aggregate as a random process. The non-uniformity of dust grains is represented by two randomly mixed distinct phases, i.e. spherical refractory sub-units covered by ice mantles and vacuum. Dust grains form in the cool expanding ambient around solar-type evolved stars, novae and supernovae. ${ }^{1}$ Dust is then injected into the local ambient interstellar medium and distributed by shock waves over a very large scale through the Galaxy. During this period, dust cycles several times between dense and diffuse clouds, thus allowing efficient processing by means of UV radiation. ${ }^{4}$ The results of this work show that the interstellar accreted material within aggregates delivered to the protoplanetary nebula is likely to be radical in nature. As a consequence, if the interiors of composite grains are actually chemical factories, the resulting complex products are completely dependent on the local physical conditions of the protosolar cloud. ${ }^{1}$

References

1 Dorschner J., Henning Th. A. \& A. Rv. (1995), 6, 271.

${ }^{2}$ Duley W. W. MNRAS (2000), 319, 791.

${ }^{3}$ Ehrenfreund P. Charnley S. B. ARA and A. (2000), 38, 427.

${ }^{4}$ Greenberg J. M. ASS (1986), 128, 17.

\section{Adenine in mineral samples: development of a methodology based on surface enhanced Raman spectroscopy and Raman spectroscopy for picomole detection \\ C. El Amri}

Institut Jacques Monod, Laboratoire de Biochimie de l'Evolution et Adaptabilité Moléculaire, 2, Place Jussieu Tour 43, 75251 Paris, Cedex 5, France,E-mail: elamri@ijm.jussieu.fr

Study of interactions of biological macromolecules with mineral surfaces are of crucial interest either for understanding primeval metabolic processes or for detection of traces of living activities. Also, the exploration of Mars requires perfecting fast methods, highly resolvent, nondestructive, for either in situ analysis and for samples returning analysis on earth. A prerequisite for implementation on real samples is the development of a rational methodology based on mineral models to which a controlled proportion of nucleic acids or monomers will be added. Another stake is to detect the smallest quantities. Surface Enhanced Raman spectroscopy (SERS) and Raman microscopy are techniques of choice to study samples slightly concentrated - one can detect ten to twelve moles. SERS also gives information on the structure of molecules adsorbed on an adapted surface, for example silver. We begin to study several model minerals such as clay, meteorite, etc. Study of nucleic acids transfer from the mineral phase to the colloidal phase (for SERS) has been carried out, and we determined the threshold concentration for each type of minerals. Here, we present the results concerning the adsorption and detection of the adenine nucleic based on a series of minerals including clay and meteorite. Results show that a combination of SERS effect and Raman microscopy is essential for an optimal detection of up to 10-3 picomoles. $\mathrm{pH}$ effects on adsorption and detection are also presented.

\section{Uracil dosimeter in simulated extraterrestrial conditions \\ A. Bérces}

MTA-SE Research Group for Biophysics, Hungarian Academy of Sciences, 8H-1444 Budapest, P.O. Box 263, Hungary,

E-mail:berces@puskin.sote.hu

In the Laboratory polycrystalline uracil thin layer detector has been developed for UV dosimetry on the Earth's surface. Exponential response of the uracil polycrystal has been detected both by absorption spectroscopy and measurements of the refractive index under the influence of terrestrial solar radiation or using UV-C sources. In UV biological dosimetry the UV dose scale is additive starting at a value of zero according to the definition of CIE (Technical Report TC-6-18). The biological dose can be defined by a measured end-effect. In our dosimeter exposed to natural (terrestrial) UV radiation the proportion of pyrimidin photo products among the total photo products is smaller than 0.1 and the linear correlation between the biological and physical dose is higher than 0.9. According to the experimental data this linear relationship is often not valid. We observed that UV radiation did not only induce dimerization but shorter wavelengths caused monomerization of pyrimidin dimers. Performing the irradiation in an oxygen-free environment and using a Deuterium lamp as UV source, we could increase monomerization against dimerisation thus the DNA-based dosimetrys additive rule is not fulfilled in these conditions. In this study we will demonstrate those non-linear experiments, which constitute the basis of our biological experiments on the International Space Station, ISS

\section{Study by UV-visible spectrometry of gas/solid interactions in Titan's simulated atmosphere J.-M. Bernard(1), P. Coll(1), A. Jolly(1), Y. Bénilan(1),}

G. Cernogora(2), F. Raulin(1)

(1) LISA Universités Paris 12 et Paris 7 UMR 7583, France, E-mail: bernard@lisa.univ-paris12.fr,CMC61 avenue du Général de Gaulle 94010 CRETEIL Cedex, France; (2) S.A., UMR 7620, France The atmospheric chemistry on Titan is reproduced during laboratory simulation experiments over several years. The device we developed at LISA led to the identification of 70 gaseous compounds (46 hydrocarbons and 24 nitrogenous compounds), while the chemical composition of the solid phase is still not known in spite of IR spectrometry and pyrolysis analysis. These simulations are based on an initial mixture of $\mathrm{N}_{2} / \mathrm{CH}_{4}$, submitted to a glow discharge, at continuous flow and at low temperature in order to simulate as well as possible Titan's atmosphere. The highlighting identification of $\mathrm{C}_{4} \mathrm{~N}_{2}$, detected on Titan but never identified previously in experimental simulations, validates the representation of the LISA experiment. The aim of the project presented today is an experimental study of the reactional environment where the solid phase is produced, considered as an analogue of Titan's aerosols using in situ UV-visible spectrometry. The analysis of the evolution of the compounds (molecules/radicals/ions) present in the reactor will allow the identification of those responsible for the building of the solid phase, at the gas/solid interface. A second step will be the modelling of the mechanisms taking place in the reactor, which are very badly known at the present time. The final stage of this work will be to use a full theoretical model of plasma chemistry, still being developed. We will present today the first results obtained by emission spectroscopy, during an experimental simulation of Titan's atmosphere, and point out the decreasing of some compounds in parallel to the formation of the solid phase. 
In-situ geological and biological analysis of planetary surfaces: a proposal for an integrated instrument A. Del Bianco(1), R. Bertran(2), C. Kolb(3), G. Kurat(4),

H. Lammer(3), V. Lazić(5), H. Voraberger(6)

(1) CTR AG, Europastr. 4, A-9524 Villach/St. Magdalen, Austria, E-mail: alessandro.delbianco@ctr.at; (2) von Hoerner and Sulger GmbH, Schlossplatz 8, D-68723 Schwetzingen, Germany; (3) Space Research Institute, Department of Extraterrestrial Space Physics, Austrian Academy of Sciences, Schmiedlstr. 6, A-8042 Graz, Austria;

(4) Museum of Natural History, Burgring 7, A-1014 Vienna, Austria;

(5) ENEA, Via Enrico Fermi 45, I-00044 Frascati, Italy;

(6) Joanneum Research FmbH, Institute for Chemical Process

Development and Control, Steyrergasse 17, A-8010 Graz, Austria

The European Space Agency Exobiology Science Team has recently reviewed the problems linked to the search of extraterrestrial life. In their final report, ${ }^{1}$ a package of instruments devoted to the exploration of planetary surfaces from the point of view of exobiology was recommended. In the scientific payload, both a microscope for general examination of the samples and a Raman spectrometer for the geological/biological characterization have been recommended as first priority instruments with the baseline set to Beagle-2 and Mars Surveyor, respectively. Furthermore, an elemental analysis of the sample will be desirable to fully characterize the components of the sample. This latter analysis can be carried out by Laser-Induced Breakdown Spectroscopy (LIBS) technique. A system engineering study of these three devices leads to the conclusion that they share several common subsystems and therefore an integrated instrument will present the following engineering advantages: reduction of mass and size with respect to separate instruments. Single focussing procedure for all the instruments (1) Single delivery of sample (2) Molecular and elemental analysis automatically carried out in the same position (3) Thermal control of a single unit (4) On the other hand, it is possible to foresee the following drawbacks (5) The system feasibility is under evaluation, therefore the development stage of such an instrument is in delay with respect to existing or under development systems (6) The mechanical and optical architecture is slightly more complex than for a single instrument (7) Furthermore, the compactness and low power consumption of integrated instrument can have interesting applications in the following fields: Precise farming; to characterize in real time and in situ some of the components of soils (e.g. phosphates) to optimize the quantity of fertiliser needed with benefits for the economy and for an effective environment protection policy. Non-destructive analysis of works of art; to analyse the composition of ancient glasses and paintings to help determine the correct restoration procedure for Semiconductor industry. To determine the stresses and strains of Silicon crystals for quality control. Plastic industry; for quality control and process monitoring of the Oil industry; for quality control and analysis of impurities in the process stream.

Reference

${ }^{1}$ A. Brack, B. Fitton, F. Raulin, ESA coordinators, Exobiology in the Solar System and the Search for Life on Mars, European Space Agency, SP-1231 (1999).

\section{Magnetic field reversals on Earth: possible implications for the biosphere}

H. Biernat(1), N. V. Erkaiev(2), D. F. Vogl(1), S. Mühlbacher(1), H. Lammer(1), S. C. Manrubia(3), F. Selsis(3), T. Penz(1)

(1) Department for Extraterrestrial Physics, Space Research Institute, Austrian Academy of Sciences, Schmiedlstr. 6, A-8042 Graz, Austria, E-mail: helfried.biernat@oeaw.ac.at; (2) Institute of Computational Modelling, Russian Academy of Sciences, 660036 Krasnoyarsk 36, Russia; (3) Centro de Astrobiologia (CSIC-INTA) Associated to NASA Astrobiology Institute, Spain, Carretera de Ajalvir,

km 428850 Torrejon de Ardoz, Madrid, Spain

Direct observations of the Earth's magnetic field span only a few centuries, paleomagnetic measurements provide the essential means for investigating the long-term behaviour of the field over much longer time scales. The generation and behaviour of the geomagnetic field reversals are closely tied to other planetary environmental processes, for example enhanced surface particle exposure, enhanced solar wind interaction with the atmosphere and possible related super volcanoes and climate change scenarios. Consequently a discussion of fundamental aspects of Earth's paleomagnetic research is related to effects of the biosphere and life. We discuss the behaviour of the Earth's dipole field and its reversals, give possible explanations of reversals, and show the classes of magnetospheres caused by different field orientations of the planets internal field. We study further the solar wind interaction with the Earth atmosphere during the time when the protecting Earth magnetic field is very weak. Further we compare and discuss magnetic reversal time scales with biodiversity and the outbreak of super volcanoes, which affected the biosphere.

\section{The number of habitable planets in the Milky Way over cosmological time scales \\ W. von Bloh \\ Potsdam Institute for Climate Impact Research, PO Box 6012 03, 14412 Potsdam, Germany,E-mail:bloh@pik-potsdam.de}

A general modelling scheme for assessing the suitability for life on any Earth-like extra-solar planet is presented. This approach is based on an integrated Earth system analysis in order to calculate the habitable zone in main-sequence-star planetary systems. Within this model the evolution of the habitable zone over geological time scales can be calculated straightforwardly and allows an estimate of the probability that an Earth-like planet is within the habitable zone around an extra-solar planetary system. A new attempt to estimate the formation rate of Earth-like planets is applied to calculate the average number of habitable planets in the Milky Way as a function of time. This approach is based on a quantitative determination of metallicity from star formation rates as an ingredient for the formation of Earth-like planets. We find that there were a maximum number of habitable planets at the time of Earth's origin. Thus, interstellar panspermia was most probable at that time and may have kick-started life on our planet.

\section{Polymerisation of amino acids thioesters on mineral surfaces in dilute solution}

A. Brack, B. Barbier, M. Bertrand, A. Chabin, F. Westall

Centre de Biophysique Moléculaire, CNRS, Rue Charle Sadron 45071, Orléans, Cedex02, France, E-mail: brack@cnrs-orleans.fr

Polymerization of amino acid thioethylesters in $3 \mathrm{mM}$ aqueous solutions in the presence of mineral surfaces is described. The standard conditions of polymerization have been determined for Leu-SEt in the presence of $\mathrm{CdS}$ by optimizing the concentration of $\mathrm{CdS}$, the $\mathrm{pH}$ and the temperature. The addition of $\mathrm{CuCl}_{2}$ did not markedly improved the polymerization performances. Wet and dry cycles increased the length of the oligomers formed up to the heptapeptide. Different amino-acids and mineral surfaces were tested. The best results were obtained with leucine thioethylester in the presence of CdS or ZnS. Polymerization reactions were also run in the presence of samples collected at the Rainbow hydrothermal system site on the Atlantic Ridge.

\section{A test for the search for vegetation on extra-solar planets \\ Danielle Briot \\ Observatoire de Paris, 92195 Meudon, France, \\ E-mail:danielle.briot@obspm.fr}

The search for life on extra-solar planets can be made - indirectly by the detection of by-products of biological activity, such as oxygen and methane, and directly by 'vegetation-like' spectral features. They should both be associated with detection of water, assumed to be a prerequisite for the development of life. After a review of the compared advantages and disadvantages of both approaches (artefacts, universality etc), we focus on a test of the detectability of vegetation on Earth seen as a single point: the detection of vegetation in the spectrum of the global Earth on the lunar surface, or Earthshine. We review the first results obtained by different groups, new ongoing observations and future plans. 
Thermodynamic parameters of water related properties of amino acids at low temperature ranges M. G. Cacace(1), A. Sada(2), Andras Dér(3), J. J. Ramsden(4), P. Falciani(5), R. Pallini(5)

(1) Institute for the Study of Nanostructured Materials CNR., Bologna Unit, Siena, Italy, E-mail: cacace@unisi.it; (2) Institute of Food Science and Technology, CNR Avellino, Italy; (3) Institute of Biophysics, Biological Research Center Hungarian Academy of Sciences, Szeged, Hungary; (4) Collegium Basilea, Institute of Advanced Studies, Basel, Switzerland; (5) Finmeccanica SpAAlenia Avionics Div.-Off. Galileo Unit, Campi Bisenzio, Florence and

University of Siena, Siena, Italy

Cometary ices are sites for the formation and preservation of organic compounds relating to the origin of life. Some of the chemical species, which have been detected in comets, have also been detected in the interstellar medium, indicating a probable relationship between interstellar dust and gas clouds and comets. Even if the presence of life in comets is unlikely, model experiments have shown that the molecules contained in comets may give rise to the formation of the biochemical compounds which are necessary for life, such as amino acids, nucleic acids precursors, monosaccharides, etc. and the capture of cometary matter by the primitive Earth is considered essential for the development of life on this planet. Low temperature simulations of prebiotic synthetic processes thought to occur on ice-covered planets and satellites such as Europa indicate that components necessary for the origin of life may have been produced in substantial yields under icy conditions. We have developed a method to measure thermodynamic parameters of partition coefficients for relevant low molecular weight precursors, such as the naturally occurring amino acids. The experimental set up allows the determination of $\mathrm{K}_{\mathrm{d}}$ of relevant solutes in conditions akin to gel permeation chromatography experiments. This information is useful to ascertain the characteristics of the interaction of these molecules with organized, structured water layers, such as the ice surface of interstellar dust or comets. The ranking of the temperature dependence of such interactions is not related to their hydrophilic-hydrophobic character but gives rise to a unique experimentally derived scale for the twenty naturally occurring amino acids. This information may well contribute to our understanding of the chemistry and physics of biochemical compounds at surfaces and at temperatures corresponding to extreme environments.

\section{About the chemical composition of carriers of the Unidentified Infrared Bands (UIBs) and protoplanetary emission spectra recorded from certain astronomical objects}

F. Cataldo(1), Y. Keheyan(2), D. Heymann(3)

(1) Società Lupi Chemical Research Institute, Via Casilina 1626/A, 00133 Rome, Italy, E-mail: cdcata@flashnet.it; (2) Istitutodi Metodologie Chimiche, CNR, Monterotondo Stazione, 00016, Rome, Italy,E-mail:yeghis.keheyan@mlib.cnr.it; (3) Department of Earth Science, Rice University MS-Box 126, P.O.B. 1892, Houston, TX77251-1892,Houston,Texas, USA, E-mail:dieter@rice.edu In this communication we present the basic concept that the pure PAHs (Polycyclic Aromatic Hydrocarbons) can be considered only the ideal carriers of the UIBs (Unidentified Infrared Bands), the emission spectra coming from a large variety of astronomical objects. Instead we have proposed $^{1}$ that the carriers of UIBs and of proto-planetary nebulae (PPNe) emission spectra are much more complex molecular mixtures also possessing complex chemical structures comparable to certain fractions obtained from the petroleum refining processes. The demonstration of our proposal is based on the comparison between the emission spectra recorded from the protoplanetary nebulae (PPNe) IRAS $22272+5435^{2}$ and the infrared absorption spectra of certain 'heavy' petroleum fractions. It is shown that the best match with the reference spectrum is achieved by highly aromatic petroleum fractions having also a kerogenic nature. Furthermore, it introduced the concept of 'statistical structural analysis' and 'average statistical molecule' to describe extremely complex organic mixtures like those composing petroleum fraction, coal and coal fraction and hence also the protoplanetary nebulae.

References

${ }^{1}$ F. Cataldo, Y. Keheyan and D. Heyamnn, International Journal of Astrobiology (2002), in press.

${ }^{2}$ B. J. Hrivnak, K. Volk, S. Kwok, Astrophysical. Journal (2000), $535,275$.

Impact-shocked rocks as a micro-habitat for life C. S. Cockell(1), D. Fike(2)

(1) British Antarctic Survey (BAS) High Cross, Madingley Road, CambridgeCB13AR,E-mail: csco@bas.ac.uk; (2) Scott Polar Research Institute, Lensfield Road, Cambridge, UK

Impact-induced shock and heating is found to increase the surface area for bacterial colonization by 25 times in gneiss from the Haughton impact structure in Canada. We have characterized two types of microbial communities that inhabit these rocks in preference to low $(<5 \mathrm{GPa})$ or unshocked gneiss. Heterotrophic bacteria that match by BLAST to known polar genera are found to inhabit the interior of examined rocks up to $10 \mathrm{~cm}$ in diameter. These eubacteria probably use organics that leach into the rocks in snowmelt and themselves are probably carried through impact-induced micro-fractures into the centre of the rocks. The second community of interest are phototrophic organisms, specifically Chroococcidiopsis, a desiccation resistant, radiation resistant cyanobacterium that inhabits the near-surface region of the shocked rocks up to a depth of $5 \mathrm{~mm}$ where photosynthetically active radiation is available. These data demonstrate that impact-shocked rocks can provide a versatile habitat for a range of micro-organisms with different functional niches. We suggest that such micro-environments might be relevant to the search for extraterrestrial life and biomarkers and they offer insights into micro-habitats available on an impactshocked Archean Earth.

\section{Search for extraterrestrial life: the French} contribution between 1870 and 1950

F. Coliolo(1), F. Raulin-Cerceau(2), J. Schneider(3)

(1) Universita' di Pescara, Italie, Florence, Italy, E-mail:

coliolo@mesioc.obspm.fr; (2) Museum National d'Histoire Naturelle,

Paris, France Equipe d'Histoire des sciences, Grande Galerie de

l'Evolution, Muséum national d'Histoire naturelle 36 rue Geoffroy

St Hilaire F-75005 Paris, France, E-mail: raulin@mnhn.fr ;

(3) Observatoire de Meudon-Paris, Universita' di Pescara, Italie 78, rue de l'Ile saint louis en l'Ile, 75004 Paris, France

Since the late nineteenth century, new technologies brought major insights to research on extraterrestrial life-types: spectroscopy applied to astronomy, radio communications, extra solar planet detection, methods and first experiments of bacteria resistance in environments simulating the space conditions. At the same time, the first theories on chemical evolution leading to life have been constructed and the first hypotheses on Panspermia have been developed. One can divide the research on extraterrestrial life into three periods: (1) the era of speculations from the Greeks to the mid nineteenth century; (2) the epoch between the dawn of spectrographic astronomy (around 1870) and the turning point of the space research era; (3) the present epoch of intense preparation of space missions. In this contribution we review the history of French scientists involved in extraterrestrial life research between 1870 and the beginning of the space era. Some of them have focussed on an exhaustive investigation of the Mars surface, in particular Mars vegetation. 


\section{Extreme UV radiation protection by iron in aquatic media: biological implications in planetary enviroments \\ C. Córdoba-Jabonero(1), L. Vázquez $(1,2)$}

(1) Centro de Astrobiología CAB (CSIC-INTA) Ctra. Ajalvir, km. 4, Torrejon de Ardoz, 28850, Madrid,Spain,E-mail:cordobajc@inta.es; (2) Universidad Complutense de Madrid, Facultad de Informática, Dpto. Matemática Aplicada Ciudad Universitaria, 28040-Madrid, Spain

A radiative transfer model was used to study the penetration of solar UV radiation in aquatic extreme environments, as it was reported by the authors in a previous work, ${ }^{1}$ in which a possible radiation protection by organic and inorganic matter dissolved in water was proposed. The aim of this work is simulating experimentally this previous work in order to study in more detail the important role of inorganic matter in the UV radiation protection. Thus, we have tried to reproduce in the laboratory the extreme UV radiation conditions to study its propagation through a water layer, where inorganic matter, in particular iron ions, has been dissolved. The UV radiation protection by the iron compounds ${ }^{2}$ depends on the type of electronic state of iron in the water, i.e. depending on which state is the predominant one: $\mathrm{Fe} 2+$ or $\mathrm{Fe} 3+$. Moreover, the UV radiative properties of the acidic water in the Tinto river (Huelva, Spain), as a real case of an extremophyle aquatic environment, are also investigated. It is important to point out that the Tinto river, which is being biologically characterised by R. Amils and the Laboratory of Extremophyles of the Centro de Astrobiología (CAB), has a great variety of microorganisms (organic matter) as well as high concentrations of iron (inorganic matter). We will compare the experimental data obtained in this work with the results previously reported by using radiative transfer modelling, and discuss the limits of application, as well as the knowledge to this study contributed by each method. Finally, the biological implications, as is the reduction by iron protection of biological damage under extreme UV radiation levels, will be investigated. An extrapolation to the Martian environment, where the water presence is expected to be possible in the past history of Mars, ${ }^{3}$ and the UV radiation conditions are very extreme, ${ }^{4,5}$ will be considered.

\section{References}

${ }^{1}$ Córdoba-Jabonero C., et al. (2001). Protection for life in aquatic media under extreme solar ultraviolet radiation conditions. Proc. First European Workshop on Exo/Astrobiology, Frascati (Italy), May 21-23 (2001), ESA SP-496, 325-328.

${ }^{2}$ Kumar et al. (1996). UVB shielding and the role of $\mathrm{FeCl}_{3}$ and certain cyanobacterial pigment. Photochem. Photobiol. 63, 321-325.

${ }^{3}$ Carr, M. H., (1996). Wateron Mars (Oxford University Press, New York), 229.

${ }^{4}$ Cockell, C. S. et al. (2000). The ultraviolet environment of Mars: biological implications, past, present and future. Icarus, 146, 343-359.

${ }^{5}$ Córdoba-Jabonero C. et al. (2002). EUV and UV transmission in the Martian Atmosphere: biological and geological implications (submitted to Planetary and Space Science).

\section{Teaching exobiology by means of a multimedia system \\ B. Corrado \\ Astronomy Department, University of Bologna, Via Ranzani 1, I-40127 Bologna, Italy, E-mail: bartolini@bo.astro.it}

At Bologna University Exobiology is a part of the 'Physics of the Planets' course. In the last three years, after some usual lectures about fundamental topics, the subject has been treated with an interactive method involving the students in the improvement and updating of a CD-ROM, containing the information collected in the previous years. The main points of the method are: (1) to share the topics among the students; (2) to search information from the internet, magazines and books; (3) to select the interesting and reliable data; (4) to set in order the chosen subjects writing a first text; (5) to discuss the text with all the students of the class obtaining a revised version; (6) to iterate this process until a satisfactory version is reached. For where exobiology is concerned, due to its interdisciplinary character, the hypertext has been submitted to experts of zoology and biology. The main points concerned are: (1) basic characteristics of the life; (2) life in extreme environments; (3) life without Sun; (4) panspermia; (5) possibility of life on Mars and Europa. This method of teaching is very efficient and appreciated by the students.

\section{Carbon chemistry and the Diffuse Interstellar Bands in the Magellanic clouds \\ N. Cox(1), P. Ehrenfreund(2), J. Cami(3), J. Jimenez-Vicente(3),} B. H. Foing(4), L. Kaper(1), A. Van Der Meer(1), L. D'Hendecourt(5), J. Maier(6), F. Salama(7), P. Sarre(8), T. Snow(9),

P. Sonnentrucker (10)

(1) Astronomical Institute 'Anton Pannekoek', Amsterdam, The Netherlands, E-mail: ncox@science.uva.nl; (2) Leiden Observatory, Leiden, The Netherlands; (3) Kapteyn Institute, Groningen, The Netherlands; (4) ESA-ESTEC-SO, Noordwijk, The Netherlands; (5) Institut d'Astrophysique Spatiale, Orsay, France; (6) Institute for Physical Chemistry, Basel, Switzerland; (7) Space Science Division, NASA Ames Research Center, Moffett Field, USA; (8) School of Chemistry, Univ. of Nottingham, Nottingham, UK; (9) Center for Astrophysics and Space Astronomy, Univ. of Colorado, Boulder, USA; (10) Department of Physics and Astronomy, J.H. University,

Baltimore, USA

Investigating the distribution and properties of Diffuse Interstellar Bands (DIBs) and thus indirectly that of complex carbonaceous molecules is important for our understanding of the properties of the Interstellar Medium (ISM). Linking DIB observations with other properties of the ISM in various galaxies, like the Magellanic Clouds, enables us to trace and understand the distribution of complex molecules in interstellar clouds, the raw material of stars and planets. We have observed with the VLT at unprecedented (UVES) spectral resolution the absorption spectrum towards reddened hot stars in the Magellanic Clouds over the wavelength range 3500-10500 Angstrom. This range covers the strong transitions usually associated with neutral and charged large carbon molecules of varying sizes and structures. We report the first detection of diffuse interstellar bands (DIBs), e.g. 5780 and 5797 Angstrom in the Small Magellanic Cloud (SMC). And also the variation of the 6284 Angstrom DIB toward several targets in the Large Magellanic Cloud (LMC). The variation of DIBs in the Magellanic Clouds compared to galactic targets seems to be governed by a combination of the different chemical processes prevailing in low metallicity regions and the local environmental (e.g. UV radiation) conditions. These results provide insights into the universal pathways of carbon chemistry throughout the universe.

\section{Robust molecular imprinted polymer thin-films for an astrobiology biomimetic sensor array \\ D. C. Cullen}

Cranfield Biotechnology Centre, Cranfield University Silsoe Bedfordshire MK45 4DT UK, E-mail:d.cullen@cranfield.ac.uk

A key objective within astrobiology for future planetary lander missions is the detection of biomarkers - molecules whose presence indicates the existence of either current or extinct life. Additionally, there is growing interest to detect diverse organic molecules present in situ on comets and related bodies. In general, past and current approaches to the challenge of biomarker and organic molecule detection have utilized or proposed spectroscopic and/or chromatographic instrument packages that have a number of features that limit their desirability including: (1) often complex molecular species cannot be identified unambiguously, (2) detection of a limited range or class of molecules for a given single instrument and (3) mass and power consumption limitations. Recent developments in the micro-fabrication of sensor arrays, micro-fluidics and micro-electromechanical systems, artificial molecular recognition systems (biomimetics) and methods to integrate the aforementioned components make possible the realization of a compact, robust artificial molecular-recognition sensor array for biomarkers, especially organic biomarkers, as an alternative to the approaches described above. We have proposed the development of a biomimetic sensor array based on robust artificial molecular receptors comprised of molecular 
imprinted polymer recognition systems (MIPs). ${ }^{1}$ MIPs are expected to be robust receptors within the extreme environments encountered during lander missions compared to commonly used biological molecular recognition molecules such as antibodies. Within this context, we have progressed the proof-of-concept demonstration of the proposed biomimetic sensor array. (1) Protocols for the deposition of MIP thin-films (10-100 nm thick) on transducer surfaces using photo-polymerization and surface-tethered photo-initiators have been implemented for both aqueous and non-aqueous deposition systems. The resultant MIP films have been physically characterized with AFM and surface plasmon resonance studies and exhibit the desired homogeneity. (2) Exposure studies of MIPs in extreme environments relevant to lander missions such as particle and gamma radiations, UV radiations equivalent to 50 days on Mars, thermal vacuum cycling $\left(+10{ }^{\circ} \mathrm{C}\right.$ to $\left.-100{ }^{\circ} \mathrm{C}\right)$, acoustic energy, standard sterilization procedures (heat treatment/ hydrogen peroxide plasma) have been initiated to confirm the suitability and robustness of MIPs for astrobiology applications.

Reference

${ }^{1}$ Proposed biomimetic molecular sensor array for astrobiology applications, D. C. Cullen, W. D. Grant, S. Piletsky and M. R. Sims, Proceedings of The First European Workshop on Exo/Astro-Biology (2001), 329-332.

\section{Crystallization of the outer surface (s-layer) glycoprotein of the mesophilic bacterium bacillus spaericus and the hyperthermophilic archaeon methanothermus fervidus}

T. Debaerdemaeker(1), C. Evrard(2), J.-P. Declercq(2), H. Claus(3),

E. $\operatorname{Akça(3),~H.~König(3)~}$

(1) Sektion Röntgen- und Elektronenbeugung, Universität Ulm, Albert Einstein Allee 11,89081 Ulm, Germany, E-mail:

tony.debaerdemaeker@chemie.uni-ulm.de; (2) Laboratoire de Chimie et de Cristallographie, Université Catholique deLouvain, 1348 Louvain-la-Neuve, Belgium; (3) Institut für Mikrobiologie und Weinforschung, Johannes Gutenberg-Universität D-55099 Mainz, Germany

For some years our interest has been focussed on the determination of the three-dimensional crystal structure of S-layer (glyco-)proteins from methanogenic Archaea of the genus Methanothermus and mesophilic, thermophilic and extremely thermophilic species of the genus Methanococcus in order to obtain a better understanding of the principles of molecular stabilization of extremely thermophilic cell surface proteins. For comparative studies, Methanococci are of special interest, since members of this genus are closely related to each other and their habitats range from mesophilic to extremely thermophilic environments. Crystals from the S-layer glycoprotein of Methanothermus fervidus were grown under microgravity conditions during the flight STS-95 of the space shuttle Discovery. We extended our research also to the domain Bacteria in order to find out whether the stabilization of these thermophilic S-layer proteins is based on the same principles as in the domain Archaea. In this case members of the genus Bacillus were chosen because the species of this genus are closely related to each other as is the case with the Methanococci and mesophilic as well as thermo-philic species. In the meantime, we were also able to grow crystals of the S-layer glycoprotein of Bacillus sphaericus.

\section{Utilisation of inorganic substrata and production of organic substances by non-differentiated plant tissue cultures cultivated in low magnetic field}

C. Dobrot(1), E. Harsan(2), A. Balogh(2), M. Yamasahita(3),

R. Tetean(4), D. Iosif(4)

(1) Faculty of Biology and Geology, Babes Bolyai University,

1 Kogalniceanu St. 3400 Cluj-Napoca, Romania, E-mail:

crisd@bioge.ubbcluj.ro; (2) Horticultural Research Station, 3400 Cluj,

Romania; (3) Institute of Space and Astronautical Science, Yoshinodai, Sagamihara Kanagawa 229-8510, Japan; (4) Babes Bolyai University, Faculty of Physics, 3400 Cluj, Romania

In order to survey how magnetic fields affect the mineral nutrition and the primary metabolism of plant tissue cultures, the vegetal material was incubated under low magnetic field (LMF) and also in normal geomagnetic environment. Calluses of soyabean (Glycine max), sunflower (Helianthus annuus) and potato (Solanum tuberosum) induced from shoot tip were incubated in a magnetic shield box and in a control box for 48 hours, respectively for 20 days. Growth of calluses, the mineral content $\left(\mathrm{NO}_{3}{ }^{-}, \mathrm{NO}_{2}{ }^{-}, \mathrm{NH}_{4}{ }^{+}, \mathrm{PO}_{4}{ }^{3-}\right.$, and $\left.\mathrm{Fe}_{2}{ }^{+}\right)$of culture medium, carbohydrates, lipids and water content of tissues were determined. At short time exposure the growth of potato calluses was stimulated but not the other two types of calluses. The nitrogen utilization, in both oxidized and reduced form, is higher for all the tissues maintained in the shield box. The phosphorus utilization is higher for the sunflower tissues and lower for potato and soyabean tissues. At long-time exposure the nitrogen utilization remain higher in shielding conditions but the phosphorus utilization decrease under the control. The iron accumulation in the shielded tissues is higher than in the control one. The lipids content decreases and the carbohydrates content increases compared to control, suggesting the occurrence of a gluconeogenesis process.

\section{Influence of planetary dynamos in a possible early Earth and Mars parallel biogeological evolution} A. G. Fairén(1), J. Ruiz(2), M. A. de Pablo(3), E. R. Uceda(4), J. M. Dohm(5), V. R. Baker(5)

(1) Seminar on Planetary Sciences, UCM, Universidad Complutense de Madrid 28040-Madrid, Spain, E-mail: Fairen.ag@terra.es; (2) Departamento de Geodinàmica, UCM, Universidad Complutense de Madrid 28040-Madrid, Spain, E-mail: jv.ruiz@teleline.es; (3) Escuela Superior de Cc. Experimentales y Tecnología. URJC, Universidad Rey Juan Carlos, Madrid, Spain, E-mail: depablo@ geo.ucm.es; (4) Servicio de Endocrinología, Hospital Ramón y Cajal, Carretera de Colmenar, km. 1. Madrid, Spain,E-mail: eruceda@, hotmail.com; (5) Department of Hydrology and Water Resources, University of Arizona, Tucson, Arizona 85721, USA

The Martian magnetic field could have had deep implications in both the stability of the planet atmosphere and hydrosphere, and in the evolution of live forms, due to the shielding against the cosmic rays and the lighter particles from solar wind that such a field provides. These are capable of eroding a planetary atmosphere entirely, and of preventing any possible cellular organization becoming impossible for the persistence of life. However, a large amount of evidence suggests that Martian former atmosphere was much thicker than contemporary (e.g., the orbital capture of Phobos), and that there were long-lasting liquid water masses on the surface (e.g., runoff and outflow channels, lowlands topography). Thus, it seems important for the possible biological history of Mars to delimitate the geological time in which the magnetic field was operative on the planet. The magnetic anomalies detected in some regions by MGS could provide an initial estimation, as they are representative of the time in which the magnetic field was operative in Mars, before Hellas and Argyre forming impact basins, roughly $4 \mathrm{Ga}$. Besides, the magnetization time of magnetic minerals in the carbonates in the interior of ALH84001 suggests that the Martian magnetic dynamo was active prior to $3.9 \mathrm{Ga}$. So, both Martian and Earth magnetospheres could have constituted a protection good enough for the stability of their respective atmospheres and oceans, and for life to develop, in the earlier hundreds of millions of years of the Solar System history. In fact, the lack of an effective shielding against ultraviolet radiation, due to the absence of free atmospheric oxygen and consequently ozone, was not a problem for life to rise, to evolve and to extend all over the Earth; and it may have not been a problem on Mars either, under the same conditions. The maintenance of such an atmosphere and hydrosphere capable of supporting life requires, as well as the above-mentioned magnetic field, any process of carbonate recycling, as it occurs on Earth through plate tectonics. On Mars, extensive volcanic episodes (e.g., Tharsis rise) could have accounted for carbonate reinjection into the atmosphere; besides, it is possible that Martian plate tectonism were active not only at the same time as dynamo, but even for some time after. This possible parallel biogeological evolution of early Earth and Mars has been recently supported by the analysis of magnetite chains within carbonates in the interior of ALH84001: the magnetization intensities of the carbonates suggests an intensity for the ancient magnetic field on Mars roughly of an order of 
magnitude of that at the surface of Earth today, sufficient for magnetotaxis by the bacteria whose magnetofossils have been reported in the meteorite. In the absence of terrestrial contamination, these fossils imply a Martian biological evolution following the same biochemical and physiological parameters as Earth, allowing not only the old debate about conditions for life origin, but further debates on similar original responses in morphology, structure and habitat selection wherever it was on the terrestrial planet in which it evolves.

\section{The Martian highland/lowland boundary as the ancient planet shoreline: implications for surface water evolution}

A. G. Fairén(1), J. M. Dohm(2), M. A. de Pablo(3), V. R. Baker(2)

(1) Seminar on Planetary Sciences, Universidad Complutense de Madrid 28040 Madrid, Spain, E-mail:Fairen.ag@terra.es;

(2) Department of Hydrology and Water Resources, University of Arizona Tucson, Arizona 85721,USA,E-mail:jmd@hwr.arizona.edu;

(3) Escuela Superior de Cc. Experimentales y Tecnología, Universidad Rey Juan Carlos I. Madrid, Spain,E-mail: depablo@geo.ucm.es

Liquid water on the surface of Mars throughout the planets recorded history has shaped the landscape into a number of distinct morphologic types, including prominent features such as the circum-Chryse and recently identified northwestern slope valleys (NSVs) outflow channel systems and the extremely flat northern plains topography at the distal reaches of these outflow channel systems. A Noachian, Europe-sized drainage basin/aquifer system in eastern Tharsis has been recently proposed to source the magmatic-triggered outburst floods that sculpted the circum-Chryse and NSVs channel systems, entrained boulders, rock, and sediment during passage, and ponded to form various hypothesized oceans, seas, and lakes. A synthesis of the geological information (e.g., stratigraphic, paleotectonic, erosional, etc.) indicates Late Noachian, Early Hesperian, Late Hesperian, and possibly Amazonian pulses of Tharsis superplume activity that could have contributed floodwaters that resulted in the geomorphologic and topographic expression of the northern plains that arguably indicates the past existence of oceans and lakes and brief excursions in climate (tens of thousands of years) from the standard dry cold desert conditions. The water supply of the Noachian basin/aquifer system, however, is thought to have dwindled over time with each endogenic-driven event (insufficient recharge), and thus floods and water bodies are expected to be progressively smaller with time. The process would include a first Late Noachian stage, portrayed by the Martian highland/lowland boundary, when a large ocean covered all Mars septentrional third. Evidence for the Martian boundary as the ancient planets ocean shoreline will be presented, in order to test the viability of the historically debated original great ocean. We base our analysis on: (1) crater counting in the region separating Contacts 1 and 2, which points to an intermediate age for this area; (2) paleotectonic information, including pre-oceanic tectonic structural fabrics, such as structurally controlled cliffs in the original Oceanus Borealis, and post tectonically controlled elevation adjustments to the putative Contact 1 related to the evolution of Tharsis and/or other elevational adjustments related to possible plate tectonism, volcanic, wind and/or glacial activity, or impact cratering; (3) estimates of total water capacity below Contact 1 $\left(9.6 \times 10 \mathrm{~km}^{3}\right)$, which lies between the minimum value for the one that flowed through the outflow channels $\left(0.6 \times 10^{7} \mathrm{~km}^{3}\right)$ and the maximum water content estimated for regolith $\left(5\right.$ to $\left.20 \times 10^{7} \mathrm{~km}^{3}\right)$; and (4) the approximated values of the hypothesized smaller water-masses, whose associated sedimentary deposits and water resources could have altered the primitive geomorphological features of Contact 1 . After a dry intermediate period, a secondary, Early Hesperian ocean would have extend over the deeper areas in the lowlands. During the Late Hesperian, major effusive volcanism, which resulted in the early development of the Tharsis Montes and Olympus Mons shield volcanoes and extensive lava flow fields on the southeast flank of Syria Planum, could have resulted in yet another inset to the larger Late Noachian ocean. Finally, a collection of lakes could have formed when Mars came through another thermal cycle, after Hesperian-age outflow channels formation. The inner ocean and lakes shorelines would be greatly subdued/modified because of the magmatic-triggered perturbations in flooding and resulting bodies of waters as well as from pervasive Amazonian wind modification. This hypothesis holds on the assumption that total water amount on Mars would have been progressively reduced in time, due to the planet's low gravity, impact erosion and hydrodynamic escape; or, alternatively, to the fact that less water was able to be mobilized from subsurface reservoirs.

\section{Description of a Mars-simulation facility}

K. Finster(1), A.-M. Jensen(1), A. Hansen(1), B. Aa. Lomstein(1), P. Nornberg(2), J. Merrison(3)

(1) Department of Microbial Ecology, Institute of Biological Sciences, University of Aarhus Building 540, Ny Munkegade, DK-8000 C,

Denmark,E-mail:biokf@biology.au.dk; (2) Department of Geology, University of Aarhus, Denmark; (3) Department of Physics and Astronomy, University of Aarhus, Denmark

The parallels between Earth's and Mars' early history resulted in the hypothesis that Mars is a site where extraterrestrial life might have and/ or may still occur(ed). To test the hypothesis, sample return missions are planned by NASA and ESA. However, the enormous economic costs and the logistic challenges of these missions make earth-based model facilities inevitable. For this reason a Mars simulator system was constructed in which atmospheric composition, temperature, pressure and radiation can be regulated independently. The simulator has an inner diameter of $30 \mathrm{~cm}$ and is $35 \mathrm{~cm}$ high. Samples can be introduced and removed from the simulator through a sluice without disturbing ongoing experiments. In addition, the simulator is equipped with temperature and resistor sensors by which the temperature and the vapour pressure of water within studied specimen can be followed continuously. Detailed studies on the effect of Mars environmental conditions on the survival and the activity of microorganisms and their metabolic products can be performed in the system. Thus, the simulation facility will contribute to our understanding of the possibility of life on Mars and it may advise in-situ sites, which are particularly promising for the search for extraterrestrial life. This study is part of an interdisciplinary project, which addresses the potential for life on Mars.

\section{Energy dissipation of possible Titan lightning strokes as production mechanism for prebiotic molecules G. Fischer(1), T. Tokano(2), W. Macher(1), H. Lammer(1),} H. O. Rucker(1)

(1) Department for Extraterrestrial Physics, Space Research Institute, Austrian Academy of Sciences, Schmiedlstr. 6, A-8042, Graz, Austria, E-mail: georg.fischer@oeaw.ac.at; (2) Institut für Raumsimulation, DLR, D-51170 Köln, Germany

The search for lightning on Saturn's satellite Titan is one of the scientific targets of the Cassini/Huygens mission. Although Voyager 1 did not detect any radio emissions attributed to Titan lightning during its fly-by in November 1980, one cannot generally rule out their existence due to low flash rates or ionospheric radiation blockade. We have recently developed a thundercloud model in Titan's troposphere favouring the existence of Titan lightning due to charged clouds causing temporary electric fields sufficiently strong to initiate cloud-to-ground lightning strokes. ${ }^{2}$ In the present investigation we estimate the amount of energy dissipation of such cloud-to-ground strokes by electrostatic energy considerations similar to those of reference number one. ${ }^{1}$ Whereas lightning strokes produce a transient hot channel in which high-temperature chemistry takes place, various new molecules can be formed. Lightning can be regarded as a possible energy source for the formation of some trace species including prebiotic molecules in Titan's troposphere.

References

${ }^{1}$ Cooray V., Energy dissipation in lightning flashes, J. Geophys. Res., 102, D17, 21401-21410 (1997).

2 Tokano T., G. J. Molina-Cuberos, H. Lammer, and W. Stumptner, Modelling of thunderclouds and lightning generation on Titan, Planet. Space Sci., 49, 539-560 (2001). 
Formation of thermophilic streamer biosignatures in Yellowstone National Park, USA

J.-F. Flot(1,2), S. L. Cady(3)

(1) Department of Chemistry, Biology and Marine Science University of the Ryukyus, Senbaru 1 Nishihara Okinawa 903-0213, Japan, E-mail:flot@rip.ens-cachan.fr; (2) Département Biochimie-Génie Biologique, Ecole Normale Supérieure de Cachan 61, avenue du President-Wilson, 94235 Cachan Cedex, France; (3) Geomicrobiology Electron Microscopy Laboratory, Department of Geology, Portland State University, 1721 SW Broadway, 17 Cramer Hall, Portland, OR9720,USA,E-mail:cadys@pdx.edu

Thermophilic communities that thrive in the hot springs and geysers of Yellowstone National Park are thought to represent analogues of some of the Earth's earliest inhabitants. Among these bacterial consortia are those that form microbial mat streamers, a fascinating example of survival in a microenvironment characterized by both high temperature (up to $86^{\circ} \mathrm{C}$ ) and aggressive hydrodynamics. Recognition of morphological biosignatures in ancient or extraterrestrial rock samples is often hindered by the lack of detailed, micro-scale observations available for present-day terrestrial analogues. We investigated the architecture of several types of microbial streamers collected from silica-depositing springs and outflow channels in Yellowstone National Park. Optical, scanning and transmission electron microscopy, and confocal laser scanning microscopy were used as complementary methods for investigating their architecture and earliest stages of silicification in a spatially integrated approach. We found that these communities, regardless of their microbial components, all exhibit the same overall organization: filamentous cells are arranged in a three-dimensional network that serves as a substratum for non-filamentous microorganisms. While it was known that the macroscale attributes of streamer fabrics are preserved in hydrothermal deposits, we found that during the earliest stages of silicification their microscale architecture is still recognizable at the submicroscopic scale, allowing identification. These results establish a framework for the recognition of fossils of thermophilic communities in the rock records.

\section{Evolution of microbial populations in extreme} acidic conditions

F. Gómez(1), R. Amils(1,2)

(1) Centro de Astrobiologia (CAB INTA), Carretera de

Ajalvir Km 5.4, Torrejon de Ardoz, 28850 Madrid, Spain,

E-mail:fgomez@cbm.uam.es; (2) Centro de Biología Molecular

'Severo Ochoa' (CBM) Universidad Autonoma de Madrid,

Campus de Cantoblanco, 28049 Madrid, Spain

Living systems are able to develop and adapt to extremely harsh conditions and also to radically transform the environment in which they thrive. Recently the discovery of chemolithotrophic microorganisms participating in a photo-independent carbon cycle has opened an interesting perspective to the origin and evolution of life on our planet and the search for life elsewhere in the universe. The exploration of these habitats on Earth have been limited in the past due to the constrains of the conventional microbial technologies (isolation from enrichment cultures and its phenotypic characterization). The introduction of molecular ecology techniques (DGGE and FISH) have resulted in an important improvement, specially in the characterization of extreme environments, like the chemolithotrophic-acidic ones, in which the phenotypic properties could not help much in the identification and quantification of the different microbes involved in the system. Molecular ecology techniques have been used to study the evolution of microbial populations in two acidic environments of astrobiological interest: the Tinto River and a fossil fuel in a bioleaching assay.

\section{The Tinto river, an extreme acidic environment under control of iron}

F. Gómez(1), E. González-Toril(2), N. Rodríguez(1), D. Fernández-

Remolar(1), M. A. Aguilar(1), M. Malki(2), R. Amils(1,2)

(1) Centro de Astrobiología (CAB-INTA), Carretera Ajalvir Km 5.4,

Torrejon de Ardoz, 28850 Madrid, Spain,E-mail: ramils@cbm.uam.es;

(2) Centro de Biología Molecular 'Severo Ochoa' (CBM) Universidad

Autonoma de Madrid, Campus de Cantoblanco, 28049 Madrid, Spain

The identification in the Tinto River of iron-oxidizing and iron-reducing microorganisms that can operate, not only, in aerobic conditions, but in the absence of oxygen, underlines the existence of a fully operative iron cycle in the extreme conditions found in the system. Sulphur-oxidation and also sulfur-reduction are also operative in the Tinto ecosystem. Furthermore, both cycles, the iron and the sulphur cycles, operate in an interrelated manner. The Tinto River is an ecosystem controlled by iron. Iron is not only a source of energy for iron-oxidizing prokaryotes, but also can be used as electron acceptor for anaerobic respiration. Ferric iron is responsible for the constant $\mathrm{pH}$ of the ecosystem, a property of importance for the maintenance of the outstanding level of eukaryotic diversity found in the river. Furthermore, acidic ferric iron solutions absorb harmful UV radiation, so they can give radiation protection to the microorganisms growing in its presence. All these properties are operative in the Tinto ecosystem and might help to understand the parageneses of other iron deposits not only on Earth but in other planetary systems.

\section{$\mathrm{Al}$ and $\mathrm{A2}$, two novel haloarchaeal isolates from bore cores of Alpine rock salt with similarity to strains from ancient English and Polish salt sediments C. Gruber, M. Pfaffenhuemer, G. Weidler, H. Stan-Lotter Institute of Genetics and General Biology, University of Salzburg, Hellbrunnerstr. 34, A-5020 Salzburg, Austria, E-mail: claudia.gruber@sbg.ac.at}

We had isolated previously from Permian rock salt collected in a salt mine in Bad Ischl, Austria, a halophilic archaebacterium, which was designated Halococcus salifodinae BIp. Subsequently, it was found that independently isolated strains $\mathrm{Br} 3$ (from solution-mined brine, Lostock, Cheshire, England) and BG2/2 (from a bore core from the mine of Berchtesgaden, Germany) resembled Hc. salifodinae BIp in colonial morphology, 16S rRNA sequences and other properties. ${ }^{1}$ From additional salt samples from the same site we recovered, eight years after the original isolation, several halococci which proved to be identical to Hc. salifodinae. ${ }^{1}$ These results led to our suggestion that extremely halophilic microorganisms, which inhabited the ancient hypersaline seas over Europe, might have been enclosed in salt deposits which formed upon evaporation, and might have stayed viable since then. Recently, we isolated a second novel Halococcus species and described it as Hc. dombrowskii. ${ }^{2}$ By using molecular methods we found evidence for the presence of numerous other types of haloarchaea, besides Halococcus species, ${ }^{3}$ but have not cultivated them until now. We investigated freshly drilled salt cores from a depth of about $600 \mathrm{~m}$ below surface in the salt mine of Altaussee, Austria, which were dissolved immediately in sterile water. Plating the dissolved salts on nutrient agar containing $125 \mathrm{~g} / 1$ $\mathrm{NaCl}, 160 \mathrm{~g} / 1 \mathrm{MgCl}_{2} \times 6 \mathrm{H}_{2} \mathrm{O}, 5 \mathrm{~g} / 1 \mathrm{~K}_{2} \mathrm{SO}_{4}, 0.1 \mathrm{~g} / 1 \mathrm{CaCl}_{2} \times 2 \mathrm{H}_{2} \mathrm{O}, 0.1 \%$ yeast extract, $0.1 \%$ casamino acids and $0.2 \%$ starch, we were able to isolate, following incubation for 8 weeks, two red pigmented colonies, which were cultivated for further investigation. Amplification of the $16 \mathrm{~S}$ rRNA genes by PCR followed by sequencing showed more than $99.8 \%$ sequence similarity to uncultured archaeal clones, e.g. A175 (3), and $99.7 \%$ sequence similarity to cultured haloarchaeal isolates from ancient salt deposits in England (Winsford salt mine brine) and Poland (Wieliczka salt mine brine). According to 16S rRNA gene sequences, whole cell protein patterns following SDS polyacrylamide gel electrophoresis, and restriction digestion patterns of their DNA, isolates A1 and A2 were $100 \%$ identical. They were similar in morphology (short rods) to Halobacterium salinarum and $\mathrm{Hb}$. sp. NRC-1, which were cultivated from surface waters. The results suggested that viable 
haloarchaea, besides halococci, can be isolated from Alpine salt deposits, which appear to have survived for millions of years.

\section{References}

${ }^{1}$ Stan-Lotter H, McGenity TJ, Legat A, Denner EBM, Glaser K, Stetter KO, Wanner G (1999) Very similar strains of Halococcus salifodinae are found in geographically separated Permo-Triassic salt deposits. Microbiology 145, 3565-3574.

${ }^{2}$ Stan-Lotter H, Pfaffenhuemer M, Legat A, Busse HJ, Radax C, Gruber C (2002) Halococcus dombrowskii sp. nov., an Archaeal Isolate from a Permo-Triassic Alpine Salt deposit. Int. J. System. Evol. Microbiol. (in press).

${ }^{3}$ Radax C, Gruber G, Stan-Lotter H (2001) Novel haloarchaeal 16S rRNA gene sequences from Alpine Permo-Triassic rock salt. Extremophiles 5, 221-228.

\section{The near Sun space promotes microorganisms being in an absolute substrate absence}

V. A. Gusev

Sobolev Institute for Mathematics of Siberia Division, Russian

Academy of Science, Koptyuga av. 4, Novosibirsk 630090, Russia,

E-mail:vgus@math.nsc.ru

The Sun's trajectory is scanned by a reflector telescope. ${ }^{1}$ The light-tight lid covered the entrance hole, so only electromagnetic radio waves should be came. The plastic tubes containing water suspension of E. coli microorganisms were placed on the ocular point. The telescope was fixed at the Sun's trajectory points for three minutes. After that, incubation of every tube determined quantities of living microorganisms by inoculated samples of plates containing nutrient agar. It was demonstrated that maximum living microorganisms in the tubes which were kept at the telescope, fixed on the sun-trajectory ahead $2^{\circ}$ of Sun. Why and how do microorganisms distinguish the physical properties near Sun's space? Reference

${ }^{1}$ Lavrent'ev, M. M., Gusev, V. A., et al. Registration of the real position of the Sun. Doklady Academii Nauk SSSR (Rus.). V. 315, N2, pp. 368-370 (1990).

\section{Survival and multiplication of organotrophs in the absence of organic substratum}

V. A. Gusev

Sobolev Institute for Mathematics of Siberia Division, Russian

Academy of Science, Koptyuga av. 4, Novosibirsk 630090, Russia,

E-mail:vgus@math.nsc.ru

It will be shown that the electromagnetic field is a very important component for the origin of life on the Earth. In a literal sense, we take a bath into the electromagnetic ocean. All microorganisms at all times lived and multiplied in the water are immersed in these electromagnetic fields. This has been said by ancient sage: Nature can not be empty, as it is difficult to imagine that living systems did not use this source of energy. Today it is known with certainly that solar energy is necessary for selfreproduction of phototrophs. Chemotrophs are used chemical energy of organic or inorganic origin. What will happen with these microorganisms to place them in the absolutely poor medium, i.e. is empty from any one pointed source of energy? The seemingly evident answer will be that they are die out. But experiments show that this is not always true. Over a period of years our research team investigated startling phenomenon of long-time survival rate of heterotroph microorganisms E. coli and its multiplication in absolutely poor distillate water. Essentially we have not published our data until 1998, the year before we have solved two principal problems: (1) What is the source of carbon for multiplication of microorganisms? (2) What is the source of energy for this process? Both problems have been solved and published now ${ }^{1}$.

Reference

${ }^{1}$ Gusev, V. A.: The source of energy for survival and multiplication of heterotroph microorganisms in the absence of organic substratum. Biophysics (Rus.), 46, No. 5, pp. 862-878 (2001).

\section{Origin and evolution of life on other worlds: lessons} from the history of life on Earth

L. N. Irwin(1), D. Schulze-Makuch(2)

(1) Department of Biological Sciences and Geological Sciences, University of Texas at El Paso, USA, E-mail: lirwin@utep.edu; $500 \mathrm{~W}$. University Ave. El Paso, Texas 79968, USA

A number of themes relevant to the possible course of life on other worlds are evident in the history of life on Earth. Those themes include (1) the origin of life relatively quickly under permissive physicochemical conditions, (2) conservation of form and function under static environmental conditions, (3) rapid adaptation at times of environmental crisis, (4) preponderance of microscopic and simple over macroscopic and complex forms in general, but (5) inexorable growth in complexity for a diminishing fraction of the total biomass, and (6) progressive specialization and interdependence in heterogeneous environments. Assuming the consistent operation of chemistry and physics throughout the universe, and the reasonable but unproven assumption that universal laws govern the higher-order organization of matter and energy, life on other worlds can plausibly be predicted to be (1) widespread but predominantly simple, (2) sequestered in stable environments such as the subsurface, and (3) more elaborately evolved than on Earth in rare and isolated instances.

\section{Martian basalts as a medium for carbon signature conservation \\ J. Jehlicka, F. Ota \\ Institute of Geochemistry, Mineralogy and Mineral Resources, Faculty of Science, Charles University in Prague, Albertov 612843 Prague 2, Czech Republic,E-mail: jehlicka@natur.cuni.cz}

Different basaltic rocks well-known and sampled on Earth contain a low concentration of carbon. Carbon is detected in minerals (e.g. in olivine), glasses, determined in fluid inclusions or can form fillings in fractures. In the solid state, graphite is the most frequent phase but carbonaceous material with not strictly defined structure has also been observed. Carbon with structural and microtextural state similar to the industrial so-called glass-like carbons has been described at Mítov (Bohemian Massif) from andesitic basalts cross-cutting a black shale formation of Neoproterozoic age. It is proposed that Martian basalts could be seen as an ideal medium for the conservation of precursor carbonaceous signature. Bodies of basalts can entrap carbonaceous material previously transformed due to basaltic magma heat. Later, after solidification, carbonaceous matter is very well protected against external alteration or weathering accompanied by UV radiation. If the carbonaceous material is entrapped inside closed micro- and mesopores of glass-like carbon it is isolated even better from the external influences. This is due to high chemical resistance and extremely low permeability of glass-like carbons for gases and liquids.

\section{Possible detection of lightning at Titan by the Huygens experiment HASI-PWA}

I. Jernej(1), Ö. Aydogar(1), B. P. Besser(1), P. Falkner(2), R. Grard(2), M. Hamelin(3), J. J. Lopez-Moreno(4), G. J. Molina-Cuberos(5),

K. Schwingenschuh(1), M. Fulchignoni(6)

(1) Space Research Institute, Department for Experimental Space

Research, Austrian Academy of Sciences, Schmiedlstr. 6, A-8042 Graz, Austria, E-mail: irmgard.jernej@oeaw.ac.at; (2) Research and Science Support Department, ESA/ESTEC, P.O. Box 299, NL-2200 AG Noordwijk, The Netherlands; (3) CETP Observatoire de Saint. Maur, 4 Avenue de Neptune, F-94107 Saint Maur des Fosses Cedex, France; (4) Instituto de Astrofisica de Andalucia, P.O. Box 3004, E-18080 Granada, Spain; (5) Universidad de Murcia, P.O. Box 4021,

E-30071 Murcia, Spain; (6) Université de Paris 7 and Observatoire de Paris-Meudon, 2 Place Jussieu, F-75005 Paris, France

In early 2005, the ESA probe HUYGENS will be jettisoned from the NASA orbiter CASSINI. The probe will descend on Saturn's largest moon Titan and perform in-situ measurements from an altitude of $170 \mathrm{~km}$ down to its surface. One of the six scientific instruments aboard HUYGENS is HASI, the Huygens Atmospheric Structure Instrument, which is designed to measure the physical properties characterizing the 
atmosphere of Titan. The permittivity, Wave and Altimetry Analyser (PWA), which is an integral part of HASI, is dedicated to the characterization of the electrical properties of the atmosphere and surface including the detection of lightning. PWA will perform several electric field measurements in the frequency range of 0 to $11.5 \mathrm{kHz}$. With the help of an electric antenna, PWA will search for natural emissions and measure the magnitude of static electric fields due to charge separation. The conductivity of the atmosphere and the existence of free electrons will be checked during the whole descent with a combination of quadropolar and relaxation probes. The electric field measurements are coupled with acoustic measurements, which will allow for detecting acoustic phenomena such as thunder. In a special measurement mode, PWA is looking for Schumann resonances to possibly discover the existence of lightning discharges even if they do occur far off the HUYGENS probe.

\section{Simulating Martian surface conditions}

\section{J. Jensen, J. P. Merrison, K. M. Kirch, P. Nørnberg} Department of Physics and Astronomy, University of Aarhus, Institut for Fysik og Astronomi (IFA) Aarhus Universitet Ny Munkegade, DK-8000 Aarhus C,Denmark,E-mail:jjensen@ifa.au.dk

At the Mars Simulation Laboratory at Aarhus University, Denmark we are using a recirculating wind tunnel. The tunnel can run at Martian pressure, temperature and with any desired gas. A Mars soil analogue can be injected to simulate the influence of wind born dust. The facility is used for different biological, chemical and aerodynamical experiments. Among them are tests of dust adhesion to instruments on the Beagle-2 lander and preliminary experiments on influence of the triboelectric charging of dust.

\section{Darwin: a nulling space interferometer \\ L. Kaltenegger}

Scientific Project Department, ESA/ESTEC, Postbus 299, 2200 AG

Noordwijk, The Netherlands, E-mail: lisa.kaltenegger@esa.int

The direct detection of a planet close to its parent star is challenging because the signal detected from the parent star is between 109 and 106 times brighter than the signal of a planet in the visual and IR, respectively. Future space based missions like DARWIN and TPF concentrate on the region between $6 \mathrm{~m}$ to $18 \mathrm{~m}$, a region that contains the $\mathrm{CO}_{2}, \mathrm{H}_{2} \mathrm{O}$, $\mathrm{O}_{3}$ spectral features of the atmosphere. DARWIN is implemented on several telescope spacecrafts and one beam-combining spacecraft, possibility augmented by a spacecraft dedicated to ground communication and meteorology. The beam combiner and the telescope spacecraft fly in one plane with the telescope spacecraft at equal distance from the beam combiner. The resolution of the interferometer is adjusted by changing the inter-satellite distance. DARWIN could be implemented in a wide variety of configurations, constrained by the number of telescopes and the necessary background and starlight suppression.

\section{The radiative-adiabatic model as the basis of the general climate theory for wide range environmental conditions \\ A. V. Karnaukhov \\ Institute of Cell Biophysics, Russian Academy of Sciences, Puschino \\ 142290 Moscow reg., Russia, E-mail: akarn@icb.psn.ru}

The main ideas of the radiative-adiabatic model are analysed in detail and compared with the usual radiative-convective ones. The difficulties of climate theories based on the radiative-convective models caused by basis paradigm, which suppose full reconstruction of internal thermal stream in the atmosphere (both radiation and convective) are shown. Even very high-speed multiprocessor computers are not capable of solving this non-linear and non-equilibrium problem with sufficient accuracy. That is the reason why: (1) Most of the modern models of the Earth's climate consider climate changes only over the time interval not more than 100 years in the future. (2) The estimations of increasing global temperature of the Earth differ several times from various research groups. The deviation of other climate parameter estimations is even more. (3) The instability of the calculation scheme of the climate theories based on radiative-convective models made it necessary to build individual variants of the theory for each studied planet. Now we have climate theories of the Earth, of Mars, of Venus, etc. (4) There are difficulties in oretical studies of the sharp and significant changes of the planets' climate. The realities of this problem are associated not only with probabilities of the transfer of the Earth climate to the Venus one but with evidence of the significant changes of Mars and Venus climate in the past. The main achievement of the radiative-adiabatic model is the significant simplification of the calculation scheme without sacrifice of accuracy. It made it possible to conduct analytical estimations of the main parameters of the climate system and to create a general climate theory (GCT) for wide range of environmental conditions existing on different types of planets. To illustrate these possibilities we consider the following questions: (1) Estimation of differential greenhouse effects on the Earth caused by anthropogenic impact of $\mathrm{CO}_{2}$. This is the example of a two-component $\left(\mathrm{H}_{2} \mathrm{O}\right.$ and $\left.\mathrm{CO}_{2}\right)$ greenhouse effect. (2) Estimation of the integral greenhouse effect on Venus. This is a example of a dense atmosphere and a high level of greenhouse effect. (3) Estimation of integral greenhouse effect on Mars. This is an example of a low-density atmosphere with a very low concentration of water vapour. Finally we consider some problems that should be solved on the way to a general climate theory (GCT).

\section{Laboratory simulations on complex organics on Mars I. L. ten Kate}

Sterrewacht, Leiden University, P.O. Box 9513, Niels Bohrweg 2, 2300 RA, Leiden, The Netherlands, E-mail:tenkate@strw.leidenuniv.nl The search for organic molecules and tracers of life on Mars is the future perspective of several Mars missions. The experimental research programme described here investigates the most abundant organic molecules identified in solar system bodies and beyond, which may have been exogenously delivered to the Martian surface. A vacuum chamber, located at ESTEC, NL, equipped with a solar simulator will be used to collect data on the combined effects of UV photoprocessing, atmospheric conditions and the presence/absence of oxidizing agents on organic molecules. The chamber has a possibility of thermal and pressure control. A window allows the attachment of UV lamps and filters. Samples will be introduced into the chamber on a specially designed tray. Sample trays will be filled with organic molecules embedded in soil analogues, either porous or compact. During the simulation in-situ measurements are taken in the chamber, using a GCMS. Samples will be retrieved with various derivatization techniques. Thereafter the probes are subjected to various analyses, such as HPLC, spectroscopy, GC, LDMS and TOF-SIMS. The results of those simulations are part of a ground support experiment programme for future Mars missions.

\section{Possible niche migration on Mars based on the migration of water}

N. Kovacs, A. Kereszturi

Eötvös Loránd University of Sciences, H-1117 Budapest, Pazmany setany 1/C., Hungary, E-mail: kovacs.nickythita@axelero.hu,

E-mail:krub@freemail.hu

In this article we summarize our up-to-date knowledge of the climate changes on Mars regarding its consequences for possible ancient life forms. Because of the great climate variations during the evolution of Mars we can suspect a strong effect of it on the possible living organisms. We theoretically try to follow the supposed migration of possible niches and the locations of the best places to look for current or ancient life forms, and the migration style at crater lakes. In our model we suppose that the best period for the possible origin of Martian life was the warm Noachian when fluid water existed on or very near to the surface. During the turn of the Noachian/Hesperian there was a global cooling and later in the Hesperian and Amazonian great climate variations occurred. We used: (1) the global cooling of the climate (loss of atmosphere, hydrosphere, decrease of the internal heat flux) and (2) the climate fluctuations (mega outflow theory, changes in the tilt of the rotation axis, water breakups). Nobody knows that if once life appeared on Mars what 
environmental circumstances were preferred by the organisms. But it is a good approach if we suspect that the early warm and wet Noachian was a preferred environment for any possible living organisms - if y formed during this era. In this case during the climate changes the organisms followed the suitable $\mathrm{p}, \mathrm{T}$ and chemical factors - so we can consider this process as niche migration. The easiest way to search for these niches is to follow the distribution of liquid water. The liquid phase hints for certain $\mathrm{p} / \mathrm{T}$ zones and energy source which keep it in liquid phase. Because only some parts and aspects of the climate changes are known (and not in the exact chronological order of certain events) we divided our model into four steps all of them representing one phase of the changing climate. We analysed the possible global migration style of niches because of (1) global cooling, (2) shorter climate changes and (3) water breakups and (4) subsurface water migration. We presented a comprehensive model for the ancient crater lakes, which contains: sedimentation style, possible niche migration in and below a crater lake, and possible 'foods' and their distribution. As a result we get niche models for isolated crater lakes on Mars as places of possible life forms. Our conclusions could be useful for future searching programs, which look for signs of ancient life forms in crater lakes. Our model suggests too that during the global cooling and sinking of the water layer there could be a global sinking of niches. If we take into account the possible upward migration of water full of living organisms, we can count on living organisms in crater lakes. In this water it is possible to detect the living organisms stored deep in the subsurface water layer so we can call these periods 'living break ups'. These water bodies finish their 'life' at the end of the warm period or when the regional heat source ran out of energy and they froze into the cryosphere. Conclusion: the ancient surface niches can sink deeply below during the global cooling and can appear on the surface again only in warm periods, in the model the 'living warm-up' phase. Today the possible niches are in the subsurface water but if we are looking for signs of ancient organisms the model suggests an interesting way for this. The freshness of possible fossils depends mostly on the chemical environment. If we count on aggressive atmosphere and environment between the warm periods, we can count only on time since the warm period from the fossils' point of view, the older the fossils are the more disintegrated condition they are in. Based on this model the best places to look for relative intact fossils are the places of relative recent ' living warm ups'. Because it is difficult to drill deep below the surface, this model and its effect is important in the investigation of signs of ancient life on the surface. If there were any life form on Mars it is more probable that they were primitive life forms rather than evolved creatures - and in this case the chemical disintegration is a very important factor again. In our work we present models for the niche migration and for the changes in the environmental factors in and below crater lakes.

\section{Possible problematic situations for the Europa cryorobotic mission \\ A. Kereszturi}

Eötvös Loránd University of Sciences, H-1117 Budapest,

Pazmany setany 1/C., Hungary,E-mail:krub@freemail.hu

We analysed some possible dangerous and problematic situations which can take place during the descent of the Europa explorer cryorobot inside the ice crust. Our work summarizes the following: (1) consequences of the differences in the ice thickness and time of descent based on our and other workers' ice thickness estimations, (2) consequences of the tectonic movements in the crust during the descent of the cryorobot, (3) consequences of salt rich diapiric/cryomagmatic intrusions on the descent of the probe, (4) consequences of liquid water bodies inside the ice crust on the descent, (5) usage of the whole cryorobot below the ice crust as a robotic submarine.

\section{Astrobiological consequences of a possible plate recycling-like process on Titan \\ A. Kereszturi \\ Department of Physical Geography, Eötvös Loránd University of Sciences, H-1117 Budapest, Pazmany setany 1/C., Hungary, \\ E-mail:krub@freemail.hu}

The Saturnian's moon Titan is an important body from an astrobiological point of view. In its thick nitrogen atmosphere there are abiogen organic material syntheses which possibly also cause the production of simple aminoacids. Based on theoretical models below the ice crust of Titan there could be detected a liquid ocean just like that inside other giant satellites in the Solar System. In this work we analyse the possible connections between the two interesting zones: the organic matter rich surface and the liquid internal ocean. The organic 'layer': the methane from the surface disintegrates in the atmosphere and the ions and radicals can reconnect forming long chain hydrocarbons. The energy sources for this process are the UV solar radiation, the magnetospheric particle bombardment form Saturn, the cosmic radiation, the meteoritic ionization in the upper atmosphere and possible lightning. Based on the theories and some observational evidence the long chain hydrocarbons slowly accumulate on the surface. The water layer: based on the planetary evolution models there is a possible subsurface liquid water ocean inside Titan. Its thickness is around $280 \mathrm{~km}$ in the water $+5 \%$ ammonia case and $380 \mathrm{~km}$ in the water $+15 \%$ ammonia case. This layer arose from the slow cooling history of the volatile rich models for Titan during and after the core overturn. On top of the boundary there is an ice I layer, at the bottom some HP ices. Possible recent volcanic activity can contaminate the ocean with various elements from the core or they are dissolved through ancient activity. But basically we can not expect high volcanic activity today in the HP ices. The temperature in the liquid layer can be between 220 and $250 \mathrm{~K}$, the ammonia causes alkalinity. The exact $\mathrm{pH}$ depends on the ratio of the ammonia and the pressure. Possible connections: We can analyse the possible connection between the two 'layers' only with indirect methods: with thermal evolution models, analogy of other moons and modelling of the consequences of various known surface processes. The thermal evolution models suggest slow heat escape today and low global heat flux. This can cause very slow solid phase convection in the lower, less rigid part of the ice I crust. There are two giant moons as analogies with somewhat similar mass and density: Ganymede and Callisto. Signs of surface activity can be observed only on Ganymede as tectonism, global expansion and cryovolcanism. Very little is known about the surface features of Titan. The up to date results are only reflectance spectrums and albedo maps, which can be best modelled by a frozen water ice crust covered with organic matter with somewhere liquid hydrocarbons. We analysed the following possibilities: fractures and faults, cryovolcanism, meteorite impacts, block faulting and subsidence, solid state convection which can cause mix between the two important layers. From an astrobiological point of view it is interesting to analyse the possible connections between the organic matter rich surface and the sub-ice water ocean. Today we know no one direct sign suggests any case of recycling, but based on the somewhat similar Ganymede it is possible that some kind of cryovolcanic activity happened not long ago on Titan too. The Cassini/Huygens programme possibly can help in this question. The flybys will help to determine the quadrupole moments and exact mass, size, and density of Titan. The atmospheric and surface chemistry, elemental abundances and oxygen isotope ratios can help just like the indirect results regarding the topography or the age of the surface. Another possibility is that the suspected subsurface ocean generates a changeable magnetic field like the Galilean-moons. In this case the ionosphere of Titan can make it more difficult to observe these phenomena. 


\section{Astrobiological potentials of brown dwarfs}

A. Kereszturi

Department of Physical Geography, Eötvös Loránd University of Sciences, H-1117 Budapest, Pazmany setany 1/C., Hungary,

E-mail:krub@freemail.hu

In the last few years several brown dwarfs were discovered and based on the statistical assumptions they are a frequent type of celestial bodies in the Galaxy. We analyse the astrobiological potential of brown dwarfs in this article based on their atmospheric $p / T$ environment, chemistry, energy sources, internal environments of brown dwarfs and their evolution style. The mass of all the brown dwarfs is below 0.08 solar masses. Because this is a wide mass range we cannot count on in similar environments on different brown dwarfs. In this article we summarize the astrobiological potential of some brown dwarfs of different mass categories according to the following factors. Our work summarizes the following: (1) $\mathrm{P} / \mathrm{T}$ environment in the atmosphere, models of brown dwarfs' atmospheres are useful to look for the stability of liquid water phase. We can count for pressure values based on simple models for different mass dwarfs. The temperature is more difficult because the decreasing internal heat flux during their life cause different temperatures. We can take this into account with thermal evolution models too. We can define sinking 'habitable zones' or layers in the atmosphere of brown dwarfs. (2) We analyse the chemistry of the environments: based on the spectroscopic measurements, we have just a few data on elementary abundance in the atmospheres. Based on the theoretical models of brown dwarfs forming during stellar birth-like process. In this case we cannot count on greater metal content than inside the normal stars. It means in the atmosphere of brown dwarfs there is very low metal concentration. (3) Energy sources: We can count on the following energy sources on a brown dwarf: Internal heat from contraction is a very important energy source. Based on the analogy of the giant planets in the Solar System the internal heat can play a role in the atmospheric currents and chemistry. The magnetospheres are important factors too: based on the theoretical models there are strong magnetospheres of brown dwarfs, which could be an energy source for certain organisms. External radiation: several brown dwarfs are members of stellar systems. In this case their star's radiation can give energy into their atmosphere. The effectivity of this radiation depends on the opacity of the atmosphere above the water cloud layer. Lightning and sparks in the atmosphere (just like, for example, on Jupiter), other possible energy sources with smaller significance: cosmic radiation, interaction with planets/moons orbiting around them, meteoric ionization. Conclusion: It is widely accepted that the planetary environment for example of the Earth has great effect on the biosphere on it. There are more possible effects if we are working on a galactic scale. So it is important to look around the possible environments of brown dwarfs in the Galaxy. Based on our up-to-date knowledge, brown dwarfs form at the same places as the stars. We can count on the birth of dwarfs in star birth clouds. During and after their birth there are similar effects on them just like on the early planetary systems (perturbation from near stars, high energy radiation from high mass stars).

\section{'Drake matrix' and 'Drake diagrams': new versions of the Drake formulae \\ A. Kereszturi \\ Department of Physical Geography, Eötvös Loránd University of Sciences, H-1117 Budapest, Pazmany setany 1/C., Hungary, \\ E-mail:krub@freemail.hu}

The various versions of the Drake formulae summarize the components regarding the possibility of life and intelligence beyond Earth in our galaxy. In the last ten years there have been several important discoveries (exo-planets, extremophiles, the ocean of Europa, the reconstruction of ancient surface conditions of Earth and Mars etc.) connected with exobiology. The Drake formulae summarize in a simple way our up-todate knowledge too, but it is useful to try to extend the summarization in other ways. In this work a matrix shaped overview is published summarizing the exobiological discoveries regarding the possibility of extraterrestrial life and includes some diagrams showing in graphic forms certain parts of the matrix. The matrix: In the matrix we tried to analyse only the most simple basic requirements which were necessary for the origin of life on Earth: the chemical circumstances (metal content, molecular stability, existence of liquid water), energy source (radioactive, tidal, electromagnetic) and time length of these circumstances. It is difficult to give exact parameters to all these components, so in this article only a theoretical approach is summarized. We would like to fill this matrix with more exact physical parameters in the future - only after this will we be able to give numeric results. All these components were analysed for different objects from the interstellar grains to main sequence stars. The components were characterized by marks $(0$ is the worst and 5 is the best) or short text. Results: If we took into account only the near surface circumstances, we can analyse the same characteristics with triangle diagrams. We can approach the metal content (ratio of heavier elements than hydrogen and helium) with the bulk density, the stability of molecules with the temperature (higher temperature causes the disintegration of the molecules), and the energy production. In this diagram we can see only the surface or near surface characteristics of these bodies. There is evidence from the giant ice moons in the solar system that under the surface circumstances would be better for possible life than on the surface. The best approach to the critical circumstances is the presence of liquid water. The water shows the presence of heavier elements than hydrogen and helium (with oxygen), good temperature/pressure zones and the existence of energy to keep it in liquid form. We can make a $p / T$ diagram of the water phases and mark the $p / T$ curves for different bodies from theoretical models of its interior. There are good places for possible life in the liquid phase but it is not enough for a body to cross this area because it needs enough water content to appear in the liquid phase.

\section{Spectroscopic determination of the chemical environment in the Martian regolith}

C. Kolb(1), H. Lammer(1), H. S. Voraberger(2), A. Bizzarri(2), A. Del Bianco(3)

(1) Department for Extraterrestrial Physics, Space Research Institute, Austrian Academy of Sciences, Schmiedlstr. 6, A-8042 Graz, Austria,

E-mail:christoph.kolb@oeaw.ac.at,helmut.lammer@oeaw.ac.at;

(2) Joanneum Research, Institute of Chemical Process Development and Control, Steyerergasse 17, A-8010 Graz, Austria, E-mail: hannes.voraberger@joanneum.at, alessandro.bizzarri@joanneum.at, (3) CTR AG Technologiepark Villach, Europastr. 4, A-9524 St Magdalen/Villach, Austria

The analysis of the Martian upper surface layer by NASA's Viking lander and theoretical studies of the Martian atmosphere-surfaceinteraction suggest that the soil is highly chemically reactive. Based on laboratory experiments these should be a result of intense interactions between the regolith and the atmosphere under solar UV radiation. Of particular importance in this context are oxidants, adsorbed on Martian mineral-surfaces (hydrogen peroxide, superoxide ions, hydroxide radicals, ozone, etc.). These species are important for oxidizing reactions at the Martian surface (oxidizing inorganic but also organic matter) and for the evolution of the Martian water cycle - now and in the past Furthermore, because at least some of them are ions, they can cause limitations of electromagnetic subsurface-sounding-techniques for future Mars exploration vehicles. We present a very effective way to detect these oxidant-species and adsorbed water-films, in the application of Infrared and Raman spectroscopy. A sophisticated combination of both guarantees a maximum of information (e.g. two-dimensional spectroscopy). Moreover, the range of mid infrared is also useful for investigating the first order frequency-bands of functional groups within organic matter and the mineral substrate. 


\section{Adsorption-experiments under Martian conditions by means of in-situ Thermo-Gravimetry \\ C. Kolb(1,2), R. Abart(2), B. Sauseng(3)}

(1) Department for Extraterrestrial Physics, Space Research Institute, Austrian Academy of Sciences, Schmiedlstr. 6, A-8042 Graz, Austria,

E-mail: christoph.kolb@oeaw.ac.at; (2) Institute for Mineralogy and Petrology, University of Graz, Universittsplatz 2, A-8010 Graz,

Austria,E-mail:rainer.abart@unibas.ch; (3) NETZSCH

Gerätebau GmbH, Hallwang bei Salzburg, Austria,

E-mail:rosalz@ngb.netzsch.com,www.ngb.netzsch.com

Adsorption-experiments under Martian conditions by means of in-situ Thermo-Gravimetry, DRIFT-spectroscopy and MS in the laboratory. It was found in previous experimental studies under Martian conditions that the chemical reactivity of the Martian surface is related to the absorbate-chemistry on the mineral-surfaces within the Martian soil. Spectroscopic methods provide powerful tools for the determination of the sorbate and sorbent chemistry in both ground-based experiments and during future Mars-Missions at the Martian surface. A comprehensive method in a Mars environmental simulation facility is introduced and its application to Mars-research is discussed.

\section{Simulating the early solar radiation environment: $\mathrm{X}$-ray radiation damage experiments}

H. Lammer(1), A. Hickel(2), M.-G. Tehrany(1,3), A. Hanslmeier(3), I. Ribas(4,5), E Guinan(5)

(1) Department of Extraterrestrial Physics, Space Research Institute, Austrian Academy of Sciences, Schmiedlstr. 6, A-8042 Graz, Austria, E-mail: helmut.lammer@oeaw.ac.at; (2) Institute for Biophysics and $X$-Ray Structure Research, Austrian Academy of Sciences, Schmiedlstr. 6, A-8042Graz, Austria, E-mail: andrea.hickel@oeaw.ac.at;

(3) Institute for Meteorology, Geophysics and Astrophysics, University of Graz, Halbärthgasse 1, A-8010 Graz, Austria, E-mail: arnold.hanslmeier@kfunigraz.ac.at; (4) Departament d'Astronomia i Meteorologia, Universitat de Barcelona, Av. Diagonal 647,08028 Barcelona, Spain,E-mail:iribas@am.ub.es; (5) Department of Astronomy and Astrophysics, Villanova University, Villanova, PA 19085,USA,E-mail: guinan@ucis.vill.edu

Astrophysical studies inside the Sun-in-Time programme indicate that solar-type young stars underwent highly active phases. The studied stars are single $\mathrm{G}$-type stars with ages ranging from 70 million years to 9 billon years and have $\mathrm{X}$-ray luminosities ranging from 1 to 1000 times that of our quiet Sun. Therefore, the protoplanetary nebula and after planetary formation, the gas, dust, comets and meteoroids which traveled through interplanetary space were exposed during the period of the heavy asteroid bombardment up to several hundred million years after the birth of our solar system by these high soft X-ray fluxes. Such high X-ray intensities should have affected the organic chemistry in the planetary nebula, organic molecules on comets, meteoroids and asteroids. Forgetting an idea how such X-ray fluxes may have affected organic molecules or microorganisms, we plan to expose probes of them with X-rays in the laboratory. The X-ray-radiation damage experiments will be performed in a radiation chamber especially designed for this purpose. The SWAX system normally used at the Institute for Biophysics and $\mathrm{X}$-Ray Structure Research is constructed to get maximal scattering of the X-rays under optimal conditions, which means that the X-ray beam is collimated in this system to a rectangular profile of $20 \times 1 \mathrm{~mm}$. To radiate various biological samples over a large area, this set-up needs to be optimized to get the maximal possible area of radiation. The X-ray source, a Philips X-ray generator with a $\mathrm{Cu}$-anode, operates at $2 \mathrm{~kW}$ using Ni-filtered $\mathrm{CuK}_{\alpha}$ radiation $(1.54 \AA$ ). The sample holder will be constructed for the use of solid samples fixed on a carrier (e.g. Uracilpowder, etc.), as well as of liquid suspensions (e.g. bacterial suspensions). To avoid damage by secondary radiation, experiments will be carried out under vacuum.
The evolution of the Martian water inventory

H. Lammer(1), A. Hosseinmardi(1), H. I. M. Lichtenegger(2), C. Kolb(1), S. J. Bauer(3)

(1) Department for Extraterrestrial Physics, Space Research Institute, Austrian Academy of Sciences, Schmiedlstr. 6, A-8042 Graz, Austria, E-mail: helmut.lammer@oeaw.ac.at; (2) Department for Experimental Space Research, Space Research Institute, Austrian Academy of Sciences, Schmiedlstr. 6, A-8042 Graz, Austria; (3) Institute for Geophysics, Astrophysics and Meteorology, University of Graz, Universitätsplatz 5, A-8010 Graz, Austria

Generally it is believed that life on Earth emerged in liquid water from the processing of organic molecules. In 1969 UV spectrometers on board of NASA's Mariner 6 and 7 spacecrafts observed the Lyman-alpha dayglow of atomic hydrogen atoms in an altitude range between 200 and 24000 kilometers above the Martian surface. It is suggested that these hydrogen atoms originate from the planetary water inventory hidden in subsurface ice and permafrost. Recent observations by the High Energy Neutron Detector (HEND) instrument on board of NASA's Mars Odyssey spacecraft detected large amounts of hydrogen close to the surface around the poles and other local areas. When water evaporates from the Martian surface and subsurface ice reservoirs, its vapor reaches the upper atmosphere where solar UV radiation can break up the molecules into hydrogen and oxygen. Measurements of $\mathrm{D} / \mathrm{H}$ isotope ratios in the Martian atmospheric water vapor indicate an enrichment of D compared to $\mathrm{H}$ of about 5.5 times higher than that of the Earth ocean value. This isotope enrichment compared with geological surface features suggest that Mars lost a great amount of water during its history. The hydrogen and deuterium can escape by Jeans loss over the Martian past, where the atmosphere will be enriched in deuterium. Hydrogen can also be transformed into ions via charge exchange reactions resulting in ion, loss, not detected, because of observation capability limits of the Mariner 10 spacecraft. For getting a better idea how much water was really lost to space, we reevaluated all known escape rates and processes of hydrogen and oxygen to space by using a Monte Carlo model where we can study particles in the upper Martian atmosphere and a test particle model which involves the motion in the external electric and magnetic fields so that we can separate hydrogen atom fluxes which have their origin at the planet from those who originate in the solar wind. We use an actual observed $\mathrm{D} / \mathrm{H}$ isotope fractionation efficiency in the Martian atmosphere and our models to trace the total water loss from present to 3.5 Gyr ago. Further, we compare the detected and measured $\mathrm{D} / \mathrm{H}$ isotope ratios of Martian SNC meteoroids that were ejected from the planet during its history with the atmospheric studies and the actual HEND observations.

\section{Sputtering of surface matter from Europa}

H. Lammer(1), P. Wurz(2), I. L. ten Kate(3), R. Ruiterkamp(3)

(1) Department for Extraterrestrial Physics, Space Research Institute, Austrian Academy of Sciences, Schmiedlstr. 6, A-8042 Graz, Austria

E-mail: helmut.lammer@oeaw.ac.at; (2) Physics Institute,

University of Bern, Sidlerstr. 5, CH-3012 Bern, Switzerland,

E-mail:peter.wurz@soho.unibe.ch; (3) SacklerLaboratoryfor

Astrophysics, Leiden Observatory, P.O. Box 9513, 2300 RA Leiden,

The Netherlands, E-mail: tenkate@str.leidenuniv.nl,

ruiterka@strw.leidenuniv.nl

At the Jovian satellite Europa, the intense radiation bombardment of the surface produces a gas exosphere of sputtered molecules and volatile composition products. Only a couple of species in the exosphere have been identified: $\mathrm{O}_{2}$ from water/ice, $\mathrm{Na}$ and $\mathrm{K}$ from decomposition of hydrated minerals, and likely, $\mathrm{H}_{2} \mathrm{O}, \mathrm{SO}_{2}$ and $\mathrm{CO}_{2}$. A detailed study of the $\mathrm{Na} / \mathrm{K}$ ratio in Europa's exosphere will give a better understanding about the fractionation on freezing and up welling of subsurface ocean material. It is suggested that this exosphere must contain many other molecular species, which should be detectable, by a future space probe. This presents an exciting possibility of identifying surface constituents by detecting gas-phase products. Since the surface sputtering yields by heavy energetic ions like $\mathrm{O}^{+}$or $\mathrm{S}^{+}$are of order unity, we work to see if even heavy organic molecules embedded in the ice of Europa's 
surface can be ejected into the gas-phase and detected with a spacecraft orbiting Europa.

\section{Study of viral fitness evolution associated with serial bottleneck events}

E. Lázaro(1), C. Escarmis(1,2) S. C. Manrubia(1),

J. Pérez-Mercader(1), E. Domingo(1,2)

(1) Centro de Astrobiología (CSIC-INTA), Ctra de Ajalvir, Km 4,

Torrejón de Ardoz, 28850 Madrid, Spain, E-mail:mell@laeff.esa.es,

(2) Centro de Biologia Molecular 'Severo Ochoa' (CSIC-UAM),

Universidad, Autónoma de Madrid, Spain

All RNA viruses share several characteristics such as large population size, high mutation rate, and short generation time. As a consequence, viral populations are heterogeneous and dynamic structures composed by ensembles of different genomes evolving in response to environmental alterations. These structures are named viral quasispecies. The degree of adaptation of a viral quasispecies to a given environment is quantitated by its fitness value, which reflects the replicative capacity of the population. Experimental results with several RNA viruses indicate that large population passages often result in fitness gains while repeated bottleneck events lead to average fitness losses due to the operation of Muller's ratchet. In this study several foot-and-mouth disease virus (FMDV) clones were subjected to 130 serial plaque-to-plaque transfers to study the evolution of fitness values and nucleotide sequences. After an initial exponential decrease in fitness, a stationary state was reached, where fitness values fluctuated around an average constant value. A numerical model has been developed that describes a similar behavior of fitness evolution of clones subjected to serial bottlenecks. The statistical properties of the stationary state depend on several parameters, such as probability of advantageous versus deleterious mutations, initial fitness, and the number of replication rounds. Essential to an agreement between predictions of the model and experimental results was the inclusion in the model of the occurrence of advantageous, compensatory mutations, as experimentally observed in the course of fitness gain of FMDV and other viral systems. Comparison of the entire genomic nucleotide sequence of the virus at several plaque transfers established that during the plaque transfers the number of mutations accumulated linearly, and that the nature of the fixed mutations was very different from that of the mutations fixed during large population passages, leading to fitness gains. A statistically significant clustering of mutations at some genomic sites was observed.

\section{Morphology, growth and protein patterns of \\ halococci from Permo-Triassic rock salt}

A. Legat(1), C. Gruber(1), C. Frethem(2), H. Stan-Lotter(1)

(1) Institute of Genetics and General Biology, University of Salzburg, Hellbrunnerstr. 34, A-5020 Salzburg, Austria, E-mail:

andrea.legat@sbg.ac.at; (2) Department of Genetics, Cell Biology, and Development, 6-160 Jackson Hall, University of Minnesota,

\section{Church St. SE, Minneapolis, MN 55455, USA}

Extremely halophilic archaebacteria (haloarchaea) were isolated from Austrian Permo-Triassic rock salt. Several of these isolates were identified as Halococcus sp., according to their 16S rRNA gene sequences and chemotaxonomic characteristics., ${ }^{1,2}$ These cells grew in liquid medium and on agar plates as tetrads or larger clusters, similar to Hcc. morrhuae, as observed by scanning electron microscopy. Growth of these halococci was greatly stimulated by addition of sea salts ('instant ocean' from Sigma) or sterilized rock salt (obtained from Altaussee; Austria). Irish rock salt (IS) from a Permian deposit in Carrickfergus (Northern Ireland) was added to complex medium in different concentrations $(10 \%$ up to $90 \%$ of the total salts) and produced comparable effects, such as shorter lag phases of cultures and higher cell yields. In addition, whole cell protein patterns of Hcc. salifodinae and Hcc. dombrowskii H4 were different when cells were grown in the presence or absence of IS. These differences were dependent on the amount of added IS; it was observed that several proteins appeared to be increased with increasing amounts of IS; and a few proteins seemed to be expressed additionally. In order to enable detection of halophilic microorganisms in situ, a method for staining halococci with 4,6-diamidino-2-phenylindole (DAPI) was developed. ${ }^{3}$ The procedure contained brief exposure of cells to approximately $70 \%$ ethanol; this treatment did not influence the morphological appearance of halococci, as examined by phase contrast microscopy. DAPI staining revealed a non-staining region in the central part of cells, which suggested the absence of DNA in the center of the cells. The fact that these structures were never observed in type strains like Hcc. saccharolyticus or Hcc. morrhuae leads to the conclusion that these features may be a very distinct characteristic of some halococcal isolates from Permo-Triassic rock salt.

\section{References}

${ }^{1}$ Denner, E. B. M., McGenity, T. J., Busse, H. J., Grant, W. D., Wanner, G., Stan-Lotter, H. (1994). Halococcus salifodinae sp. nov., an archaeal isolate from an Austrian salt mine. Int. J. Syst. Bacteriol. 44, 744-780.

2 Stan-Lotter, H., Pfaffenhuemer, M., Legat, A., Busse, H. J, Radax, C., Gruber, C. (2002). Halococcus dombrowskii sp. nov., an Archaeal Isolate from a Permo-Triassic Alpine Salt deposit. Int. J. System. Evol. Microbiol. (in press).

${ }^{3}$ Kepner, R. L. and Pratt, J. R. (1994). Use of fluorochromes for direct enumeration of total bacteria in environmental samples: past and present. Microbiol. Rev. 58, 603-615.

\section{Examining the physico-chemical resistance of halobacteria with the LIVE-DEAD kit, following exposure to simulated Martian atmospheric conditions and heat}

S. Leuko(1), G. Weidler(1), C. Radax(1), A. Legat(1), N. Kömle(2), G. Kargl(2), H. Stan-Lotter(1)

(1) Institute of Genetics and General Biology, University of Salzburg, Hellbrunnerstr.34, A-5020 Salzburg, Austria, E-mail: sleuko@ yahoo.de; (2) Department of Extraterrestrial Physics, Space Research Institute, Academy of Sciences, Schmiedlstr. 6, A-8042 Graz, Austria Extremely halophilic archaebacteria (halobacteria) are found today in hypersaline surface waters, such as the brines in solar salterns, or the Dead Sea. However, from alpine rock salt of Permo-Triassic age, several species of halobacteria were isolated during the last years., ${ }^{1,2}$ Halobacteria are not known to produce spores or dormant forms; thus it remains enigmatic how they survived in the salt sediments. Extraterrestrial halite has been detected in meteorites from Mars and from the asteroids; in addition, the Jovian moon Europa is thought to contain a salty ocean. Therefore halobacteria would be useful model organisms when considering the search for extraterrestrial life. We are developing experimental protocols to evaluate the effects of physico-chemical stress factors on halobacteria, in particular present-day Martian conditions. But the effect of higher temperatures is also of interest, since Mars may have been warmer in the past, and the Alpine salt sediments are known to have experienced local temperature peaks. Cells of Halococcus dombrowskii, a novel coccoid isolate from Austrian rock salt, ${ }^{2}$ were grown to an optical density of 0.9 or 0.09 , respectively. For some experiments, Halobacterium sp. NRC-1 was used. The growth medium contained up to $4 \mathrm{M} \mathrm{NaCl}, 0.1 \%$ yeast extract, $0.1 \%$ casamino acids, and sterilized rock salt. ${ }^{2}$ Aliquots of cultures were kept at minus $70{ }^{\circ} \mathrm{C}$ for several days, or freeze-dried in a lyophilizer, or incubated at temperatures of $50^{\circ}, 60^{\circ}$, $70^{\circ}$ and $80^{\circ} \mathrm{C}$ for 24 hours, respectively. In addition, exposure experiments of halobacterial cells in a liquid nitrogen cooled Martian simulation chamber were begun at the Space Simulation Facility of the Space Research Institute of the Austrian Academy of Science in Graz (see also Poster by Weidler et al.). Survival of cells was evaluated by determining colony-forming units and by examination of cellular morphology by phase contrast and fluorescence microscopy, following staining with the LIVE-DEAD kit (Molecular Probes). This kit consists of two dye substances allowing a discrimination between damaged or intact cell membranes, which is reflected in the appearance of red or green fluorescence. Results indicated that the LIVE-DEAD kit can be successfully used for halobacteria, i.e. in the presence of $4 \mathrm{M} \mathrm{NaCl}$, although it was developed for tests at low ionic strength. Data will be presented which 
show that Hc. dombrowskii survived deep freezing, higher temperatures and Martian atmospheric conditions generally somewhat better than Hb. sp. NRC-1, suggesting perhaps a certain protective action of its cell envelope.

\section{References}

${ }^{1}$ Stan-Lotter H, McGenity TJ, Legat A, Denner EBM, Glaser K, Stetter KO, Wanner G (1999) Very similar strains of Halococcus salifodinae are found in geographically separated Permo-Triassic salt deposits. Microbiology 145, 3565-3574.

${ }^{2}$ Stan-Lotter H, Pfaffenhuemer M, Legat A, Busse HJ, Radax C, Gruber C (2002) Halococcus dombrowskii sp. nov., an Archaeal Isolate from a Permo-Triassic Alpine Salt deposit. Int. J. System. Evol. Microbiol. (in press).

\section{Hydrothermal processing of organic molecules produced by gas-grain reactions under nebular conditions \\ J. Llorca}

Departament de Química Inorgànica, Institut d'Estudis Espacials de Catalunya, Universitat de Barcelona, Edifici Nexus, Gran Capità 2-4, 08034 Barcelona, Spain, E-mail: jordi.llorca@qi.ub.es

The organic content of carbonaceous chondrites and comets, which has been considered as a possible ingredient of primordial soups for the emergence of life, cannot be explained in terms of simple processes. Among several sources, gas-grain reactions operating in the solar nebula have been well recognized to play an important role in nebular chemistry. In addition to mineral phases and aggregates similar to those reported in interplanetary dust particles of cometary origin, gas-grain reactions carried out in the laboratory between model metal grains and nebular-type gas led to the formation of simple organic molecules, such as saturated and unsaturated hydrocarbons and thiols, as well as polymeric residues. In this work, the subsequent alteration of these organic materials under hydrothermal conditions, which have been likely to occur in carbonaceous chondrites parent bodies and comets, is experimentally addressed. It has been found that the transformation of alkenes to alcohols and carboxylic acids and thiols to sulfonic acids takes place at $375-575 \mathrm{~K}$ and $10-100 \mathrm{kPa}$ in the presence of phyllosilicates similar to those found in CI and CM carbonaceous chondrites. In contrast to this, if the hydrothermal processing is carried out in the presence of metallic surfaces or crushed ordinary chondrites or $\mathrm{CV}$ carbonaceous chondrites (Allende), the transformation of the original organic molecules takes place to a much lesser degree. It is concluded that the hydrothermal alteration of carbonaceous chondrite parent bodies, which resulted in the extensive formation of phyllosilicates, and probably comets was accompanied by an increase of complexity of their organic content.

\section{Astrobiology versus SETI: exploring the past versus exploring the future \\ C. Maccone}

International Academy of Astronautics (IAA), Via Martorelli 4310155 Torino (TO), Italy, E-mail: clmaccon@libero.it

The current flourishing of Astrobiological studies is an achievement of contemporary science. Astrobiology, in fact, aims at unifying into a single body of knowledge many scattered contributions that previously were investigated in rather separated branches of science, and this merging into a new single field can only be of help in making further scientific progress. Yet Astrobiology appears to have one basic limitation: it is concerned only with the scientific understanding of past events. Even if this study of the past is made with an eye to the future, yet Astrobiology can hardly call itself a science of the future. A science of the future does not exist, may claim some narrow-minded scientists. In this paper, however, we would like to contend that the future of humankind might have been some alien civilization in the past! It is thus necessary for humankind to know whether Alien Civilizations (necessarily more advanced than us) live in the Galaxy, and this is just SETI, the Search for Extra Terrestrial Intelligence. SETI searches started up in 1959-60 even before the term Astrobiology was invented to study the past and the present of life in the universe. Thus, SETI is a bolder science than Astrobiology itself, and SETI has profound implications on religious and political beliefs that account for why the political lobbies of the US Congress imposed a total ban on NASA SETI searches as early as 1993. SETI scientists so far failed to prove that other extra-terrestrial civilizations exist in the galaxy or elsewhere. Yet, these scientists have already made such tremendous advances in computer technologies devoted to SETI that a scientific outline of the SETI technological achievements is given in this paper for the benefit of all the pure Astrobiologists.

\section{Lunar Farside Radio Lab for bioastronomy and radioastronomy \\ C. Maccone}

International Academy of Astronautics (IAA), Via Martorelli 43,10155

Torino(TO), Italy,E-mail: clmaccon@libero.it

The Lunar Farside Radio Lab is an international project currently being coordinated by this author on behalf of the International Academy of Astronautics (IAA). It was initiated in 1997 by the late French radioastronomer Jean Heidmann, and when he died on 3 July 2000, I, as member of the IAA, took up the project coordination. The project objective is to study how to set up a phased array (that is a computerdriven flat radiotelescope) inside crater Daedalus on the farside of the Moon, at 180 degrees of longitude east and 2 degrees below the Moon equator. A relay satellite put in a circular equatorial orbit around the Moon will keep the link with the Earth. Such a relay satellite will collect the data from Daedalus when above the farside, and download the data towards the Earth when above the nearside. Apart from many technological problems, this study also has legal implications to be studied by the International Institute of Space Law (IISL). In fact, it is felt that the Moon farside will be of such a unique benefit for bioastronomy and radioastronomy (at last free from man-made radio frequency interference) that it should be kept free from uncontrolled future commercial and military exploitation. Also, the use of the five Lagrangian points of the Earth-Moon system is studied within the framework of this Lunar Farside Radio Lab Project of the IAA. In fact, it is evident that the Lagrangian point L2 above the farside of the Moon should be kept free of noise-emitting spacecrafts, while the other points L1, L3, L4, and L5 should be utilizable for other tasks, including the planetary defence against dangerous asteroids and comets. The basic reference about this Lunar Farside Radio Lab Project of the IAA is the paper recently published $^{1}$.

Reference

${ }^{1}$ C. Maccone, IAA, Acta Astronautica, 50, 3, 185-199 (2002).

\section{Analysis of sounding antennas of the Mars Express MARSIS experiment}

W. Macher, G. Fischer, H. O. Rucker, H. Lammer, C. Kolb, B. Schraußer, G. Kargl

Department of Extraterrestrial Physics, Space Research Institute, Austrian Academy of Sciences, Schmiedlstr. 6, A-8042 Graz, Austria, E-mail: wolfgang.macher@oaw.ac.at

The MARSIS (Mars Advanced Radar for Subsurface and Ionosphere Sounding) experiment onboard Mars Express is a ground penetrating radar for the investigation of the Martian surface and subsurface structure, especially to map underground water and ice. The MARSIS antenna system consists of a primary dipole and a clutter cancellation monopole. For the evaluation of the sounding measurements an exact knowledge of the effective antenna axis of the monopole is necessary. The spacecraft body significantly perturbs the effective axis. Numerical simulations are performed to determine the deviation from the mechanical axis. The poster exhibits first results, which show the enormous influence of the solar panels and the primary dipole antenna. It is further emphasized that the electrical properties of the Martian soil will limit the penetration depth of the radar signal, so experimental investigations in space simulation laboratories with the help of Mars soil analogues are recommended. 


\section{Magnetic field reversals on Earth: Possible implications for the biosphere}

S. C. Manrubia(2), F. Selsis(3), T. Penz(1)

(1) Department for Extraterrestrial Physics, Space Research Institute, Austrian Academy of Sciences, Schmiedlstr. 6, A-8042 Graz, Austria, E-mail: helfried.biernat@oeaw.ac.at; (2) Institute of Computational Modelling, Russian Academy of Sciences, 660036 Krasnoyarsk 36, Russia; (3) Centro de Astrobiologia (CSIC-INTA) Associated to NASA Astrobiology Institute, Spain, Carretera de Ajalvir, km 428850 Torrejon de Ardoz, Madrid, Spain

Direct observations of the Earth's magnetic field span only a few centuries, paleomagnetic measurements provide the essential means for investigating the long-term behaviour of the field over much longer time scales. The generation and behaviour of the geomagnetic field reversals are closely tied to other planetary environmental processes, for example enhanced surface particle exposure, enhanced solar wind interaction with the atmosphere and possible related super volcanoes and climate change scenarios. Consequently a discussion of fundamental aspects of Earth's paleomagnetic research is related to effects of the biosphere and life. We discuss the behaviour of the Earth's dipole field and its reversals, give possible explanations of reversals, and show the classes of magnetospheres caused by different field orientations of the planets internal field. We study further the solar wind interaction with the Earth atmosphere during the time when the protecting Earth magnetic field is very weak. Further we compare and discuss magnetic reversal time scales with biodiversity and the outbreak of super volcanoes, which affected the biosphere.

\section{Preliminary programme for analysis of Mars samples}

M.-C. Maurel(1), J.-L. Counil(2) and the French CSEEM

(1) Institut Jacques Monod, Tour 43, 2 Place Jussieu 75251 Paris Cedex, France,E-mail: maurel@ijm.jussieu.fr; (2) CNES Centre de Toulouse, 18 av. Edouard Belin 31401 Toulouse Cedex4, France A multi-disciplinary committee of scientists has emerged in France since 1999, whose work is devoted to Mars sample return. Working on the assumption that key ingredients for life, liquid water and a source of energy were present on ancient Mars, groups are currently developing strategies to detect potential extinct and/or extant life. To confirm the potential controversy of discovering life, the most sensible idea before the return of samples, is to perform high sensitive in-situ analyses exploring well-defined sites from the surface and subsurface. For this purpose, a large community from geology, astrophysics, chemical and biological sciences worked to build an interdisciplinary frame, defining sites of interest, physical and chemical conditions for remote sensing and in-situ approaches, devising fast, highly resolved, and non-destructive methods. The first step of our programme is to draw up a general protocol (including recommendations according to planetary protection), then to optimize methodologies and to produce experiments under conditions mimicking those that are characteristic of the planet Mars (irradiation, temperature, pressure, hydrous potential, salts, etc.).

\section{Layered deposit on Mars: clues to past climate history \\ S. S. Mayerberger \\ UCLA IGPP/ESS, 3806 Geology Building Department of Earth and Space Sciences, Los Angeles, CA 90095-1567, USA, \\ E-mail: sabrina@ess.ucla.edu}

Determining the past climate history of Mars is essential in order to understand the planetary evolution of the red planet and possible habitats for life. Layered deposits at the Martian polar caps contain a wealth of information regarding the recent history of the planet and its volatiles. Mars Global Surveyor's Orbiter Camera (MOC) daily monitors Martian weather, dust storms, and polar cap changes. Recently released images address a number of issues relating to the physical properties of the Martian polar residual caps and associated layered deposits. Detailed quantitative analysis of those layered deposits should give a better understanding of the properties and climate history of the
Martian polar regions and in turn contribute to the determination of future landing sites.

\section{In vitro selection of adenine-dependent hairpin ribozymes \\ M. Meli \\ Institut Jaques Monod, 2 place Jussieu F-75251, Paris, Cedex 05, France, E-mail:meli@ijm.jussieu.fr}

One of the problems of the RNA world scenario is the lack of RNA functional groups which leads to the poor catalytic repertoire of ribozymes compared to proteins. To increase RNA functionalities, ribozymes can bind small exogenous reactive molecules, and handle them to help catalysis. We hypothesize that the current role of histidine in protein catalysis was performed by adenine in primeval catalysts and that RNA world ribozymes could have bound free adenine and handled it to catalyze chemical reactions. In order to test the likelihood of such a scenario, we are selecting in vitro adenine-dependent ribozymes from a population of hairpin ribozymes variants randomized in 15-20 critical positions. The study of such ribozymes could bring information about the hairpin catalytic mechanism and have evolutionary implications in our vision of the prebiotic RNA world.

\section{Enantioselective amino acid analysis in cometary matter planned for the COSAC instrument onboard Rosetta Lander \\ U. J. Meierhenrich(1), W. H.-P. Thiemann(1), F. Goesmann(2), R. Roll(2), H. Rosenbauer(2) \\ (1) Department of Physical Chemistry, Bremen University, Leobener Str.,D-28359Bremen,Germany, E-mail:mhenrich@uni-bremen.de; (2) Max-Planck-Institut für Aeronomie, Max-Planck-Str. 2, D-37189 Katlenburg-Lindau, Germany}

Due to new results of laboratory simulation experiments of interstellar and cometary matter, we have reason to assume that a cometary nucleus contains chiral organic molecules like amino acids. ${ }^{1}$ The COSAC experiment on the Rosetta Lander (the ESA mission Rosetta to comet 46P/ Wirtanen will be launched in January 2003) is dedicated to the analysis of volatile constituents of the nucleus by gas-chromatography/massspectrometry. Here, we report on chiral stationary phases suitable for performing enantioselective gas chromatography and applicable to a wide range of chiral organic compounds including amino acids, hydroxy carboxylic acids, and even hydrocarbons. ${ }^{2}$ The gas chromatographic in situ separation of enantiomers will allow the quantification of specific enantiomers and thus the determination of enantiomeric excesses which, should they be found, might be helpful to understand the well-known phenomenon of enantiomeric asymmetry of biomolecules on Earth. References

${ }^{1}$ G. M. Munoz Caro, U. J. Meierhenrich, W. A. Schutte, B. Barbier, A. Arcones Sergovia, H. Rosenbauer, W. H.-P. Thiemann, A. Brack, J. M. Greenberg, Nature 416 (2002), 403-406.

${ }^{2}$ U. J. Meierhenrich, W. H.-P. Thiemann, F. Goesmann, R. Roll, H Rosenbauer, Chirality 13 (2001), 454-457.

\section{Sites of Mars habitable by microbes}

P. Memeo, G. Coppa

Dipartimento di Astronomia, Università di Bologna, Via Ranzani 1 ,

I-40127 Bologna, Italy, E-mail:s_memeo@astbo4.bo.astro.it

The temperature of Mars surface has been measured by the Mars Pathfinder and Viking missions in the landings sites and globally for the entire planet by the TES instrument on board of the Global Mars Surveyor and by THEMIS on board of Odyssey 2001 satellites. We compare the observed values with the temperature intervals in which the different types of extremophiles can thrive, presented by Pedersen at the GeoExtreme Workshop held in Tremiti Islands in May 2001. Considering the microbial communities found in the terrestrial permafrosts, the most promising place on Mars for the life development of bacteria and archaea is the permafrost present in zones near the equator. 
Simulation results from the University of Aarhus

J. Merrison, K. Finster, H. P. Gunnlaugsson, J. Jensen, K. Kinch, B. Lomstein, R. Mugford, P. Nørnberg

Mars Simulation Laboratory Aarhus University, Ny Munkegade, bldg.520,DK-8000 Aarhus C, Denmark,E-mail:merrison@ifa.au.dk A recirculating Mars simulation windtunnel is being used to study aspects of dust transport in a well controlled reproduction of the Martian environment. Aerodynamic computer modelling is being undertaken. This allows a theoretical framework for the simulations and for quantitative comparison to experimental data from Mars. Charging of the Martian dust has previously been predicted, though not measured. The charging of a Mars analogue material is being quantitatively investigated in this simulation facility to determine its significance. Similarly the cohesive and adhesive properties of dust grains are being studied since they are of importance both to dust transport and instrument safety. Large areas of Mars are covered by atmospherically deposited dust. Transport of humidity through this layer of fine material is also being measured in a simulation environment. In addition, instrument testing is being undertaken for the forthcoming Beagle 2 lander and for the NASA rovers.

The Italian SETI Programme: recent improvements S. Montebugnoli(1), J. Monari(1), C. Bortolotti(1), A. Cattani(1), A. Maccaferri(1), G. Maccaferri(1), S. Mariotti(1), S. Poppi(1), A. Orlati(1), M. Poloni(1), M. Roma(1), A. Scalambra(1), M. Teodorani(1), C. B. Cosmovici(2), N. Damico(3), C. Maccone(4) (1) Istituto di Radioastronomia-CNR - Medicina, Bologna, Italy, E-mail:stelio@ira.cnr.it; (2) Istitutodi Scienze Cosmiche e Planetarie - CNR - Roma, Italy; (3) Osservatorio di Cagliari; (4) Centro di Astrodinamica 'G. Colombo' - Torino, Italy

SETI-Italia is one of the main activities of the Italian Bioastronomy Project, started in 1993, as Italy is the only European country joining in this venture together with the USA, Australia and Argentina. SETI (Search for Extraterrestrial Intelligence) is an observational programme devoted to the search for possible artificial monochromatic radio signals coming from an extraterrestrial technologically evolved civilization, by using existing powerful radiotelescopes. As a result of the rotation of both planets (Earth and Exoplanet), such a signal is expected to be affected by a frequency shift due to the Doppler effect. By using the 32 meters dish of the Medicina radiotelescopes we started in 1998 a systematic search with a high-resolution spectrum analyser, named SERENDIP IV, in a four-million channel configuration. In 1999 the system was upgraded to twenty-four million channels, thanks to substantial support from the SETI Institute in California. Up to now observations have been carried out in a 'piggy-back' mode within a $1.4-23.5 \mathrm{GHz}$ range. In this way the search programme exploits the $100 \%$ of the antenna allocation time completely free. It runs in parallel with the ITASEL programme too (Italian Search for Extraterrestrial Life) (see abstract by Cosmovici et al.). In this paper we will present the experimental set-up and the recent improvements in the data processing by using the KLT transform in order to search for the presence of ETI signals even over finite bandwidths.

\section{Astrobiology courses and texts \\ D. Morrison}

NASA Astrobiology Institute, NAI, Ames 240-1, Mt View, CA 94035 , USA,E-mail:dmorrison@arc.nasa.gov

The new discipline of astrobiology is quickly establishing itself in United States colleges and universities. While only a handful of these institutions are offering degree programmes or certificates in astrobiology, a much larger number see the potential to use this discipline as the basis for introductory science courses. American colleges and universities typically require all students to take at least one science course in order to graduate. These students are usually offered several competing introductory or general-education courses from which to choose. For example, every year several hundred thousand American students take an introductory, non-mathematical astronomy survey course, and nearly 20 texts compete for this market. Surveys show that more than one hundred astrobiology courses are now being planned or offered, and the first astrobiology textbooks are being published (e.g., Life in the Universe by Jeffrey Bennett, Seth Shostak, and Bruce Jakosky; and The Search for Life in the Universe (3rd edition) by Donald Goldsmith and Tobias Owen). Most of these courses emphasize the theme of life on other worlds, as suggested by these two text titles. Other schools are offering upper-division courses in astrobiology as a way to familiarize future scientists with interdisciplinary approaches. This paper reviews the contents of some of the courses and the texts that are being used. Because astrobiology is such a new field, there is as yet no consensus concerning the core content of this discipline or the topics that should be included in introductory astrobiology courses.

\section{Use of orbital missions to detect traces of life or favourable habitats on Mars \\ C. Muller \\ Belgian Institute for Space Aeronomy B.USOC, Avenue Circulaire 3, B-1180 Brussels, Belgium,E-mail: Christian.Muller@oma.be}

The remote sensing possibilities of orbiters together with their global mapping possibilities allow them to search for an inventory of characteristics of a life habitat on Mars, they allow also for a systematic search for the gases that could be indicative of life episodes as well as favourable radiation environments. The current missions are reviewed versus their capability for this objective and ideas are proposed for a specific astrobiology Mars orbiter.

\section{Permafrost and its habitants: probable model of Mars ecosystem}

K. A. Novototskaya-Vlasova(1), A. Abramov(2)

(1) Department of Soil Biology, Moscow State University, 129323 Russia, Moscow, Snezhnaya st. 14/3, app. 72, E-mail: n_ksusha@mail.ru; (2) Department of Geocryology, Moscow State University, 129323 Russia, Moscow, Snezhnaya st. 14/3, app. 72

Earth permafrost is a unique environment that preserves a lot of viable microorganisms in extreme, but stable conditions of low temperature and water activity. A detailed study of Arctic syncryogenic sediments collected nearby Laptev Sea coast (East Siberia) was performed to reveal the viability of various groups of microorganisms. Investigated layers were differed in lithology, cryogenic structure, $\mathrm{pH}$, humidity, and presence of total organic carbon and temperature level. Syncryogenic sediments attract attention due to the fact that such sediments were frozen simultaneously with their accumulation and it is suggested they were not melted just from the beginning of the formation. Thus, syncriogenic deposits present the remaining terrestrial biotope, where microorganisms can preserve viability during the period of time the sediments are in a frozen state, and may have a special interest in the comparability of the processes in terrestrial permafrost and Martian subsurface layers. Investigated Arctic sediments were formed about 10-400 thousand years ago, have the mean annual temperature ranged from -10 to $-150{ }^{\circ} \mathrm{C}$, and high water (ice) content. It was shown that microbial communities inhabiting ancient permafrost sediments could be easily resuscitated and present presumably bacterial communities. Study of taxonomic diversity of isolated bacteria revealed the abundance of non-spore forming bacteria. The same results were demonstrated by investigation of the ancient Antarctic permafrost sediments of a few million years old taken from the boreholes in the region of Dry Valleys. It is possible to assume that high survival of non-spore forming bacteria in frozen sediments can be explained by specific mechanisms of cell differentiation to dormant forms. It was shown in model experiments that under selected cultivation conditions isolated strains of non-spore forming bacteria could form thermo-resistant cells with specific ultra structure, similar to dormant forms. Such cells may be regarded as one of the reversible dormant types of non-sporeforming bacteria that can be formed in permafrost and also recognized in other substrates including extraterrestrial environment. 
The Swedish Astrobiology Network (SWAN) I A. Nummelin

Department of Electrical and Computer Engineering, Chalmers Lindholmen University College, P.O. Box 8873, SE-40272 Gothenburg, Sweden,E-mail: albert.nummelin@chl.chalmers.se

A Swedish Astrobiology Network was founded in Stockholm on 20 November 2001. The purpose of the network is to: (1) act as a base for scientific exchange between researchers involved in astrobiology, national and international, (2) promote graduate and undergraduate classes in astrobiology, (3) promote public outreach by popularization and media time, and (4) act as an interface towards national and international funding agencies. The researchers involved in SWAN display a wide range of research activities, including astronomy, astrophysics, biochemistry, biophysics, geology, geochemistry, microbiology, molecular biology and paleontology. The network acts as a sub-network to Europe's Exo/Astrobiology (EANA), but work is in progress to affiliate SWAN with the NASA Astrobiology Institute (NAI) as well. Further information is available at the SWAN webpage, www.astrobiologi.nu.

\section{Serial analysis of RNAs (SAR): a novel molecular strategy for sampling microbial population structures C. Palacios \\ Marine Biological Laboratory Josephine Bay, Paul Center for Comparative Molecular Biology and Evolution, Marine Biological Laboratory, 7 MBL Street, 02543 Woods Hole, MA, USA, \\ E-mail:palacios@mbl.edu}

The most effective means to expand our narrowed view of Earth's microbial diversity is the use of molecular techniques including the analyses of products of PCR experiments that target ribosomal RNA and other conserved universal genes. These data types provide evidence of organisms in an environment without requiring their isolation and growth in the laboratory. However, exhaustive inventories of microbial communities still remain impractical. Serial Analysis of rRNAs (SAR) is a new high-throughput technique that can sample, at the sequence level, short species-specific regions of amplified rRNAs genes from many microorganisms in a single reaction. This provides a means to rapidly and efficiently enumerate relative numbers of rDNA templates in a nucleic acid population extracted from a microbial community. We will show the results of the application of this technique to extreme environments. Our comparative studies demonstrate the power of this technique that promises to revolutionize studies of microbial population structures. The widespread application of this technology will accelerate Astrobiology studies that seek to define environmental boundaries that are compatible with the search for life on other planets, as well as the conditions conductive to life's origins and evolution.

\section{Experiment-verification test For EXPOSE in the DLR space simulation facilities \\ C. Panitz}

Institute for Aerospace Medicine, DLR, D-51170 Cologne, Germany, E-mail: corinna.panitz@dlr.de

The International Space Station will carry the EXPOSE facility. EXPOSE is now planned to be mounted on one of the four Pallet Adapters of the Columbus Module. The orientation will be chosen such that it will obtain maximum solar illumination given orbit and altitude of the ISS. The tray-like structure will accommodate among others 6 biological PI-experiments or experiment systems of the ROSE (Responses of Organisms to the Space Environment) consortium. EXPOSE will support long-term in situ studies of microbes in artificial meteorites, as well as of microbial communities from special ecological niches, such as endolithic and evaporitic ecosystems. Each compartment is either vented, i.e. open to space vacuum, or sealed and then provided with a defined gas environment. The experiment pockets will be covered by an optical filter system to control intensity and spectral range of solar UV irradiation. Control of sun exposure will be achieved by use of shutters. The launch of EXPOSE will be affected in 2004 together with the Columbus Module and is planned to stay in space for 1.5 years. The results will contribute to our understanding of the organic chemistry processes in space, the biological adaptation strategies to extreme conditions, e.g. on early Earth or Mars, and the distribution of life beyond its planet of origin. Most of the experiments are based on laboratory or field studies and are not yet adapted to the opportunities and constraints of space experimentation. Furthermore, the compatibility of the different systems needs to be determined and mission parallel ground controls are essential for the success of the EXPOSE exobiology experiments. Therefore a profound ground support program is required to support the scientific EXPOSE community for the following objectives: (1) Definition of the individual experiments under simulated space conditions; (2) Determination of the compatibility of the different experiments (e.g., organics and biology); (3) Experiment preparation and optimization under simulated space conditions; (4) Experiment sequence test (ESTs) under simulated EXPOSE environmental conditions; (5) Mission parallel ground support under simulated EXPOSE environmental conditions Flight operations - Post flight analysis (if applicable) under simulated space conditions A presentation of the work plan, requirements and constraints of the PIs and first results of selected combinations of experiments exposed to selected simulated space parameters is given. These EVT results will be essential for the final design for the EXPOSE facility.

Effects of ancient rock salt on haloarchaeal growth: Halophiles as possible model organisms for exo-/astrobiological research

M. Pfaffenhümer, C. Gruber, A. Legat, H. Stan-Lotter

Institute of Genetics and General Biology, University of Salzburg,

Hellbrunnerstraße 34, A-5020, Salzburg, Austria, E-mail:

marion.pfaffenhuemer@sbg.ac.at

Halococcus dombrowskii $\mathrm{H} 4$ was described as a novel haloarchaeal strain isolated from rock salt which is believed to have been deposited about 250 million years ago. ${ }^{1}$ The ancient rock salt sample was obtained from the salt mine near Bad Ischl, Austria. This strain has not yet been isolated from a surface location. It was also shown that a broad procaryotic diversity is apparently present in these Alpine salt sediments. ${ }^{2}$ But most of these strains have not been cultivated under laboratory conditions until now. Neutrophilic haloarchaea are usually cultured in M 2 medium, ${ }^{3}$ which contains $200 \mathrm{~g} / 1$ sodium chloride, $20 \mathrm{~g} / 1$ magnesium chloride hexahydrate, $2 \mathrm{~g} / 1$ potassium chloride, $0.2 \mathrm{~g} / 1$ calcium chloride dihydrate, $12.1 \mathrm{~g} / 1$ Tris, $0.5 \%$ yeast extract and $0.5 \%$ HyCase (hydrolyzed casein, Sheffield). The $\mathrm{pH}$ is adjusted to 7.4. Halococci grow very slowly and yield only low cell densities when cultured in this medium. During characterisation of Halococcus dombrowskii a new medium called M2S was created by addition of dissolved and sterile-filtered rock salt to standard M2 medium. The rock salt was obtained from deep drilling operations in the salt mine in Altaussee, Austria. The bore cores were dissolved in neutralized water and the saturated salt solution (approx. 32\% dissolved solids) was mixed $1: 1$ with standard M2 medium. The medium was sterilized for fifteen minutes at $121^{\circ} \mathrm{C}$. M2S showed a growth stimulating effect on all tested halococcal strains, although it contains only half of the nutritient concentration of M2. Cultures grown in M2S medium had lower doubling times and reached a greater turbidity compared to cultures grown in M2 medium. Hence more cell mass could be harvested from cultures grown in M2S medium. Generation times of fourteen hours instead of thirty hours in standard complex media M2 were observed. Furthermore changes in cell morphology and significant differences in whole cell protein patterns were recorded. The heat stable factor which effects these increases in growth could be a specific ion or a trace element. It was also examined whether this stimulating effect is reproducible within other halobacterial genera or if there are differences in growth according to the geological habitat from which the single species have been isolated. This medium appears to approach the composition of the natural habitat of halophilic archaea and is therefore of interest for astrobiological research. When haloarchaea become captured in salt crystals, they may presumably be protected against radiation, temperature and space vacuum. Especially strains from the genus Halococcus due to their rigid cell walls are predestined for studies regarding the problem of whether microorganisms can survive interplanetary transport. 


\section{References}

${ }^{1}$ Stan-Lotter H, Pfaffenhümer M, Legat A, Busse HJ, Radax C and Gruber C. Halococcus dombrowskii sp. nov., an Archaeal Isolate from Permo-Triassic Alpine Salt Deposits. International Journal of Systematic Bacteriology (in press) (2002).

${ }^{2}$ Radax C, Gruber C and Stan-Lotter H. Novel haloarchaeal 16S rRNA gene sequences from Alpine Permo-Triassic rock salt. Extremophiles: Life Under Extreme Conditions 5, 221-228 (2001).

${ }^{3}$ Tomlinson GA and Hochstein LI. Halobacterium saccharovorum sp. nov., a carbohydrate-metabolizing, extremely halophilic bacterium. Canadian Journal of Microbiology 22, 587-591 (1976).

\section{Stability of planetary orbits in double stars}

E. Pilat-Lohinger, R. Dvorak, B. Funk, F. Freistetter

Institute for Astronomy, University of Vienna, Türkenschanzstr. 17, A-1180Vienna, Austria,E-mail: lohinger@astro.univie.ac.at

The discovery of extra-solar planets in double stars led to a growing interest of stability studies of such systems, where we distinguish between two types of motion: this is the planetary- (P-) type motion, where the planet moves around both stars and the satellite- (S-) type motion, where the planet moves around one of the two stars. Up to now five planets have been discovered in binaries moving in S-type orbits. Therefore, we will present a stability study of double stars for the whole range of massratios from 0.1 to 0.9 . The numerical computations have been done in the restricted three-body problem - which means that the planet is regarded to be mass less - where we distinguish between stable and chaotic motion by means of the Fast Lyapunov Indicator. We present the variation of the stable zone by an increase of the binary's and of the planet's eccentricity. It is obvious that the planet's eccentricity influences the size of the stable zone less than that of the binaries, but we have to point out that the reduction of the stable zone is quite similar. A similar study will be shown for the double star Gliese 86, where a close moving planetary companion had been discovered. For the P-type motion we will present a stability study of inclined orbits, using again the model of the elliptic restricted three-body problem, where we increased the eccentricity of the binaries from 0 to 0.5 . To determine the stable region we used two different methods (a) long-term orbital computations and (b) the Fast Lyapunov Indicator. A comparison of the results indicates a good agreement of the two studies. In general we can say that the stable zone is shifted to larger distances from the barycentre of the two stars if the eccentricity is increased.

\section{Experiments and simulation models for the study of prebiotic chemistry in Titan's atmosphere} G. Povoden(1), H. Lammer(2), G. Grampp(3), F. Stelzer(4)

(1) Institute of Chemical Engineering Fundamentals and Plant Design, Graz University of Technology, Inffeldgasse 25, A-8010 Graz, Austria, E-mail: guarani@sbox.tu-graz.ac.at; (2)Department for Extraterrestrial Physics, Space Research Institute, Austrian Academy of Sciences, Schmiedlstr. 6, A-8042 Graz, Austria, E-mail:

helmut.lammer@oeaw.ac.at; (3) Institute of Physical and Theoretical Chemistry, Technikerstr. 4/I, A-8010 Graz, Austria, E-mail: grampp@ptc.tu-graz.ac.at; (4) Institute for Chemical Technology of Organic Materials, Graz University of Technology, Stremayrgasse 16 , A-8010Graz,Austria,E-mail:franz.stelzer@tu-graz.ac.at

The special importance of Saturn's satellite Titan lies in its primitive, chemically reducing nature of the atmosphere, which could be compared with models for the studies of the prebiological chemical evolution in the early Earth atmosphere. Our studies will give us a better understanding concerning the formation of prebiotic molecules. The first part of our project will focus on the simulation of chemical reactions that can take place on Titan, also considering the energetic inputs such as lightning discharges, UV-radiation, and micrometeorites. By using the data already obtained and the results of these simulations, we create a model about the distribution of chemical substances. The second part is the construction of a reaction chamber representing Titan's atmosphere concerning temperature, pressure and gas composition. A high frequency generator and a UV Laser will set the energetic inputs. The results of the experiments, measured by Gas Chromatography - Mass Spectrometer (GCMS) should verify the theoretical models. The NASA/ ESA Cassini/Huygens mission to the Saturn system and the exploration of Titan will give us a huge amount of information about the atmospheric chemistry, which will be used for modifying our theoretical models and the experimental set up of our reaction chamber.

\section{Microbes in rock salt: how to find out what is in there}

C. Radax, M. Pfaffenhuemer, H. Wieland, S. Rittmann, S. Leuko, G. Weidler, C. Gruber, H. Stan-Lotter

Institute of Genetics and General Biology, University of Salzburg, Hellbrunnerstr. 34, A-5020 Salzburg, Austria, E-mail:

christian.radax@sbg.ac.at

Permo-Triassic rock salt (aged 200-250 million years) was several times shown to contain living bacteria that, in most cases, belonged to the extremely halophilic Archaea. According to current hypotheses, these bacteria originate from ancient populations that became entrapped in the rock salt during its formation and persisted in the rock salt ever since. Thus, rock salt might be considered a promising model system for the search for bacterial extraterrestrial life. Currently, two different major strategies are used to obtain information about bacterial life in an environment of interest. The first strategy relies on the assumption that bacteria can be cultivated in the laboratory if they are provided suitable nutrients and physico-chemical conditions; however, due to the very heterogeneous physiological needs of different bacteria, only a small fraction of the bacteria present in a certain sample is cultivable by current methods. In the second strategy, DNA is extracted from the sample, and the Polymerase Chain Reaction (PCR) method employed to amplify genes of phylogenetic relevance from this DNA; from DNA sequences of these genes, conclusions can be drawn about the organisms in the sample. In our studies on Permo-Triassic rock salt from Bad Ischl, Austria, we followed both strategies. The results of our PCR approach indicated the presence of at least twelve distinct sequence types (phylotypes) in our samples, all of which belonged to the extremely halophilic Archaea. Two phylotypes could be assigned to cultivated members of this family; the remaining ten phylotypes appeared only remotely related to known genera of the extremely halophilic Archaea. In order to cultivate the organisms that correspond to these ten novel phylotypes, we used fifteen growth media that differed with respect to the presence and concentration of nutrients, and salt concentration. So far, approximately 100 isolates were recovered and characterized by restriction analysis or sequence analysis of $16 \mathrm{~S}$ rRNA genes, and/or comparison of whole cell protein patterns. Based on molecular analyses, the isolates formed two different groups. One group was very similar to Halococcus strains that we frequently isolated from our rock salt samples; the other group was closely correlated to one of our novel phylotypes. Based on analyses of whole cell protein patterns, the latter group could be further differentiated into two different subgroups that could not be distinguished at the molecular level. Our results indicate that, compared to the cultivation approach, the PCR approach enables us to obtain a more detailed view on the gross phylogenetic diversity of the microbes present in rock salt. On the other hand, only cultivated strains registered subtle differences between strains; in addition, questions, such as those about the mechanisms of longevity, can currently only be addressed by studying isolated strains. Thus, both strategies have to be applied in order get a more complete view of microbial life in Permo-Triassic rock salt.

\section{Studies on metal-Cyanogen complexes as prebiotic catalyst \\ S. Raj Ali, Kamaluddin}

Department of Chemistry, Indian Institute of Technology Roorkee,

Roorkee, 247 667, India, E-mail: shah7dcy@iitr.ernet.in

Processes in chemical evolution must have involved several catalysts multifunctional in nature. Our previous work has demonstrated that metal ferrocyanides posses strong adsorption affinity towards amino acids and nucleotides. We propose that double metal cyanides might have acted as an important prebiotic catalyst. Recently, we have 
synthesized a series of metal cyanogen complexes and have studied their interaction with several biomolecules of evolutionary importance. We report here interaction of aniline and $\mathrm{p}$-anisidine with chromium ferrocyanide. The interaction of aniline and p-anisidine with chromium ferrocyanide has been studied. Maximum uptake of both anilines was observed around $\mathrm{pH}$ 7. The absorption data obtained at neutral $\mathrm{pH}$ were found to follow Langmuir adsorption. Anisidine was a better adsorbate because of higher basicity. In alkaline medium $(\mathrm{pH}>8)$ both aniline and anisidine reacted with chromium ferrocyanide to give colored products. Analysis of the products by GC-MS showed benzoquinone and azobenzene as the reaction products of aniline while p-anisidine afforded a dimer. A possible reaction mechanism for the product formation in alkaline medium has been proposed. The present study suggests that metal ferrocyanides might have played an important role in the stabilization of organic molecules through their surface activity in the prebiotic condensation reactions.

\section{Kepler and the plurality of worlds \\ F. Raulin-Cerceau}

Centre Alexandre Koyré, Muséum national d'Histoire naturelle, Paris, Equipe d'Histoire des sciences, Grande Galerie de l'Evolution, Muséum national d'Histoire naturelle 36 rue Geoffroy St Hilaire F-75005 Paris, France,E-mail: raulin@mnhn.fr

German Astronomer Kepler (1571-1630) is well-known for his three laws describing the elliptic orbital movement of the planets (published in Astronomica nova - 1609 - and Epitome - 1622). In fact, this fundamental discovery is only one part of a huge astronomical work focussed on the demonstration of Nicholas Copernicus' system. As is well known, Copernicus reversed the Earth's and Sun's position in his De revolutionibus orbium coelestium (1543). Kepler firmly believed in Copernician ideas and all his life he made strenuous efforts to provide evidence to demonstrate the still debated hypotheses: the planets' position and movement around the Sun, and the Earth turning round itself. Kepler's notoriety led him to teach mathematics in Graz from 1594 to 1598, where he explained his theory of planets trajectories. One part of Kepler's work is mostly unknown. It is the matter of his posthumous book Somnium published by his son in 1634. In this last book written like fiction, Kepler clearly aimed to convince the reader that the Earth was definitely not static. Kepler chose the Moon as a new system of references to observe the planets movement, exactly as if hypothetical inhabitants of the Moon wanted to understand the astronomical phenomena occurring around them. This mental gymnastics permitted him to realize what phenomena did belong to the typical case of the Moon, and what phenomena did not. Kepler saw in this a way of setting out basic astronomical information, a didactic means to adopt Copernicus' ideas. This paper will present Kepler's pluralism-connected ideas developed in Somnium and give prominence to Kepler's tremendous efforts to demonstrate a new astronomical paradigm.

\section{The instrument MIDAS for atomic force microscopy on cometary dust}

W. Riedler(1), K. Torkar(1), H. Jeszenszky(1), J. Romstedt(2), and the MIDAS Team

(1) Space Research Institute, Department of Experimental Space Research, Austrian Academy of Sciences, Schmiedlstr. 6, A-8042 Graz, Austria,E-mail: riedler@oeaw.ac.at; (2) Research and Scientific Support Department, ESA/ESTEC, The Netherlands

The instrument MIDAS (Micro-Imaging Dust Analysis System) is part of the Rosettta Orbiter payload and will provide three-dimensional images and statistical parameters of dust particles captured from the coma of comet $\mathrm{P} / \mathrm{Wirtanen}$. The main components of the instrument are an atomic-force microscope and a dust collection and target manipulation system. The scientific requirements to resolve the expected individual building blocks of the grains and to provide adequate statistical information have been the design drivers for the instrument. The paper shows the solutions found in various technical areas to achieve the high requirements for this mission on resolution, positioning accuracy, mechanical stability, and reliability.

\section{Seas under ice: Conditions for the stability of oceans within icy worlds \\ J. Ruiz(1), A. G. Fairén(2)}

(1) Departamento de Geodinámica, Universidad Complutense de Madrid, 28040 Madrid, Spain; (2) Seminar on Planetary Sciences, Universidad Complutense de Madrid, 28040 Madrid, Spain,

E-mail:1-jv.ruiz@teleline.es 2-Fairen.ag@terra.es

The possible existence of internal oceans in some icy bodies of the outer solar system has been supported through recent years in joint observations that cover a wide range of evidence: magnetic fields in Europa, Ganymede and Callisto, perhaps induced by Jupiter's magnetic field in an electricity-conductive layer, likely salty water, near the surface; hydrated minerals on the surface of Ganymede and Europa, which suggests the presence of water on the surface, with probably an inner source, at any moment in the past; geological evidence of a motile layer a few kilometres beneath the surface of Europa; or recent resurfacing in Triton and Enceladus, suggested by the differential craterization density and distribution. But the nature of the liquid layers could be very different, although the existence of inner oceans can be common phenomena. In fact, the conditions that seem to enable the sea's stability under ice layers substantially differ in each world: they come from the ice incapability for heat transmission through convection in large satellites (for example Callisto or Ganymede), to the tidal and deformation and heating in Europa, including the addition of solutes such as ammonia or salts that could greatly lower the ice fusion point in Titan, Triton, Enceladus or even Pluto. Moreover, in the models of small size bodies, as Enceladus, the effect of the spherical geometry should not be ignored.

\section{Evolution of catalytic efficiency in terrestrial planetary environments dominated by UV-induced reactive oxidants}

S. Scher

Department of Earth and Planetary, Univeristy of California, Mars 2012 Laboratory (MC 4767), Berkeley, CA 94720, USA,

E-mail: sscher@nature.berkeley.edu

It was recently proposed that superoxide radical ions are responsible for the chemical reactivity of Martian soil. ${ }^{1}$ Formation of ultraviolet (UV)-induced reactive oxygen species under simulated Martian conditions has been established in the laboratory. ${ }^{1-3}$ Since early (pre-ozone) stages of terrestrial evolution may have closely paralleled contemporary Martian surface conditions, we examine here a model first proposed for the development of catalytic efficiency in catabolic enzymes containing iron, or in principle, other divalent metallic porphyrin prosthetic groups. ${ }^{4}$ It was reported that catalytic ability of aqueous ferric iron progressively increases over three orders of magnitude when combined with protoporphyrin, and when the iron-porphyrin group is combined with a protein moiety. ${ }^{4}$ Similar increases have been observed with copper. Before significant quantities of organic compounds could accumulate under primitive (pre-ozone) terrestrial or current Martian surface conditions dominated by UV-induced reactive oxidants, hydrated $\mathrm{Fe}, \mathrm{Cu}, \mathrm{Mn}$, or other inorganic catalysts may have evolved as precursors to cationic metalloporphyrins that mimic superoxide dismutase (SOD), peroxidase or other catalase-like enzymes. SOD enzymes catalyse the conversion of superoxide anion radicals to hydrogen peroxide and molecular oxygen. Comparative studies with contemporary prokaryotes provide evidence for structurally similar SOD with $\mathrm{Fe}, \mathrm{Cu}$, $\mathrm{Mn}$ or $\mathrm{Zn}$ in their prosthetic groups. Accordingly, evolution of efficient catalysts may represent an essential prerequisite for the origin of life on terrestrial planets.

References

1 Yen, A. S. et al. (2000). Science 289: 1909.

${ }^{2}$ Lunsford, J. H. (1973). Catal. Rev. 6: 135.

${ }^{3}$ Che, M. and Tech, A. J. (1982). Adv. Catal. 31: 77; 1983. Adv. Catal. 32: 1 .

${ }^{4}$ Calvin, M. (1956). Amer. Scientist 44: 248; (1959). Science 130: 1170. 


\section{Planetary protection: forward contamination for Mars revisited}

S. Scher

Department of Earth and Planetary Sciences University of California, University of California, Berkeley Mars 2012 Laboratory (MC 4767), Berkeley,CA94720,USA,E-mail: sscher@nature.berkeley.edu It has been postulated that the accidental introduction of terrestrial microorganisms to other planets during the course of robotic or manned exploration may impede the detection of possible indigenous organisms, and thereby obscure subsequent studies of their biology. ${ }^{1}$ Since biological contamination of Mars would be a major scientific catastrophe, we examine here recent advances in our understanding of the Martian surface that bear on this issue. Analysis of neutron flux and gamma ray emission measurements from the Mars Odyssey orbiting spacecraft provides evidence for hydrogen correlated with the presence of water ice deposits. ${ }^{2}$ These observations would appear to increase the likelihood that terrestrial microorganisms, if unintentionally introduced, may encounter environmental conditions on Mars consistent with their longterm survival. However, the presence of one or more as-yet unidentified inorganic superoxides or peroxides induced by ultraviolet (UV) radiation on the Martian surface ${ }^{3}$ may function to limit survival of introduced microbes. Under these conditions, microbes capable of producing enzymes such as superoxidases, peroxidases or other catalases capable of destroying oxygen radicals, or, in principle, other UV-induced oxidants would exhibit a selective advantage. Laboratory simulation experiments designed to select and characterize oxidant-destroying terrestrial microbes may help to resolve this issue.

\section{References}

${ }^{1}$ Packer, E., Scher, S. and Sagan, C. 1963. Icarus 2: 293.

${ }^{2}$ Bell, J. and others. www.sciencexpress.org/30 May 2002/10.1126/ science. 1074025, 1073722, 1073541, 1073616.

${ }^{3}$ Yen, A. S. et al. 2000. Science 289: 1909.

\section{Thermal properties of the Martian regolith as a measure of near-surface water abundance and distribution}

S. Scher(1), A. Aquila(1), R. Mehta(1), N. Punatar(1),

K. Wipyewski(1), M. M. Cohen(2)

(1) Department of Earth and Planetary Sciences, University of California, Mars 2012 Laboratory (MC 4767) Berkeley CA 94720, USA,E-mail:sscher@nature.berkeley.edu; (2) Advanced Projects Branch, Space Projects Division, NASA-Ames Research Center, Moffett Field, CA 94035-1000 (Mail Stop 244-14), USA

The search for water on Mars is central to our understanding of Martian geology, selection of appropriate landing sites for robotic and manned exploration, and the study of pre-existing or possible extant life on this planet. The presence of water ice on or near the Martian surface is inferred from recent observations by the National Aeronautics and Space Administration (NASA) 2001 Mars Odyssey spacecraft. Measurements of the neutron and gamma ray flux were used to map the abundance and distribution of hydrogen, suggesting large quantities of shallow subsurface water ice deposits. ${ }^{1}$ It was found that the Odyssey data for hydrogen do not distinguish between water-bearing hydrates and hydroxides. Alternatively, measurements of thermal properties of Martian surface materials may represent a more direct and independent line of evidence for determining near-surface water abundance and distribution. This approach is based on the high specific heat or thermal capacity of water and water ice to dampen or decrease diurnal, or in principle, seasonal warming and cooling rates of surface materials. Early experiments using crushed basalt to simulate the Martian regolith, subfreezing temperatures, and reduced atmospheric pressure provide preliminary evidence in support of this approach. Initial studies were designed to establish the detection limits for subsurface water. The results of these laboratory studies may provide an alternative model for reanalysis of thermal data collected from Mars Global Surveyor, 2001 Mars Odyssey, and future Mars missions, and thus facilitate highresolution mapping of Mars water abundance and distribution.

\section{Reference}

1 www.sciencexpress.org/30 May 2002/ 10.1126/science.1074025, 1073722, $1073541,1073616$.

\section{Investigations of water on Mars using Netlander electric and magnetic experiments}

K. Schwingenschuh(I), H. I. M. Lichtenegger(1), M. Menvielle(2), M. Hamelin(2), W. Magnes(1), I. Jernej(1), H. U. Eichelberger(1), C. Kolb(3), H. Lammer(3), G. Musmann(4), W. Zambelli(5)

(1) Department of Experimental Space Research, Space Research Institute, Austrian Academy of Sciences, Schmiedlstr. 6, A-8042 Graz, Austria, E-mail: konrad.schwingenschuh@oeaw.ac.at; (2) Centre d'étude des Environnements Terrestre et Planétaires, 94107 Saint Maur, France; (3) Department of Extraterrestrial Physics, Space Research Institute, Austrian Academy of Sciences, Schmiedlstr. 6, A-8042 Graz, Austria; (4) Institute of Geophysics and Meteorology, Technical University, Braunschweig, Germany; (5) Department of Communication and Wave - Propagation, Technical University, Graz, Austria

Each of the four surface stations forming the Netlander mission will include an electric and magnetic field experiment which are continuously recording electric and magnetic fields as well as the surface permittivity. Those instruments will permit for the first time the investigation of low frequency electromagnetic waves, electrical conductivity and permittivity. Electromagnetic waves might be generated by discharge phenomena in the Martian dust storms. Low frequency electromagnetic waves can excite resonances between the conducting surface and the ionosphere. Lightning caused by Martian dust storms and discharges might also influence organic chemical reactions. Further, the Netlander mission will also provide the unique opportunity to determine the electrical conductivity of the upper layers of the Martian surface. These data are important for the study of EM-subsurface sounding techniques and one can estimate the amount of water or water ice, which is crucial for the existence of life.

\section{Radiation and particle exposure of the Martian paleoatmosphere: implications for the loss of water F. Selsis(1), H. Lammer(2), I. Ribas(3,4), H. I. Lichtenegger(5),} L. Lara(6), M. G. Tehrany(2,7), A. Hanslmeier(7)

(1) Centro de Astrobiologia (CSIC/INTA) Associated to NASA Astrobiology Institute Carretera de Ajalvir, $\mathrm{km} 428850$ Torrejon de Ardoz, Madrid, Spain, E-mail: selsis@observ.u-bordeaux.fr ;

(2) Department for Extraterrestrial Physics, Space Research Institute, Austrian Academy of Sciences, Schmiedlstr. 6, A-8042 Graz, Austria, E-mail: helmut.lammer@oeaw.ac.at; (3) Departament d'Astronomia I Meteorologia, Universitat de Barcelona, Av. Diagonal 647, 08028 Barcelona, Spain; (4) Department of Astronomy and Astrophyisics, Villanova University, Villanova, PA 19085, USA; (5) Space Research Institute, Department for Experimental Space Research, Austrian Academy of Sciences, Schmiedlstr. 6, A-8010 Graz, Austria; (6) Instituto de Astrofísica de Andalucia, Camino Bajo de Huétor 24, P.O. Box 3004, 18080 Granada, Spain; (7) Institute for Geophysics, Astrophysics and Meteorology, University of Graz, Universitätsplatz 5, A-8010 Graz, Austria

Multi-wavelength studies of solar like G-type stars at several stages of their main sequence evolution indicate that our Sun may have also undergone a highly active phase in its particle and radiation evolution 3.0-4.5 Gyr ago. Detailed observations of such Sun-like stars by the ROSAT and ASCA X-ray satellites show that the X-ray luminosity may have been several hundred times higher than today. Studies of isotope anomalies in planetary atmospheres and meteorites suggest also that our early Sun underwent a very active phase after its origin that included continuous flare events and had a particle and energie irradiance several hundred times stronger than today. We investigate in our study how these high X-ray, solar EUV and particle fluxes have influenced the evolution of the Martian atmosphere. The early Martian atmosphere proves to be a very efficient shield for X-rays: with the exception of very hard $\mathrm{X}$-rays $(1<20 \mathrm{~A})$ that penetrate deeper into the atmosphere, the longer wavelength is absorbed in the atmospheric upper layers. This high 
altitude absorption results in an energy deposition at low-density levels that enhances the thermal and nonthermal escape processes already known to be driven by the high EUV radiation and solar wind particle sputtering. We also discuss the consequences of hydrodynamic escape of water from early Mars.

\section{Solar flares, generation of solar cosmic rays and their influence on biological systems}

V.S. Semenov(1), N. K. Belisheva(2), Yu. V. Tolstyh(3),

H. K. Biernat (4)

(1) Institute of Physics, University of St. Petersburg, St. Petergof,

Russia,E-mail: sem@geo.phys.spbu.ru; (2) Botanic Garden, Russian Academy of Sciences, Apatity, Russia; (3) Institute of Physics, University of St. Petersburg, St. Petergof, Russia; (4) Department of Extraterrestrial Physics, Space Research Institute, Austrian Academy of Sciences, Schmiedlstr. 6 A-8042 Graz, Austria,

E-mail:helfried.biernat@oeaw.ac.at

Reconnection is an energy conversion process which is believed to be of universal importance for astrophysical plasmas, in particular reconnection is responsible for solar flares and the generation of solar cosmic rays. An analysis of time-dependent reconnection with moving slow shocks is presented which extends the famous solution obtained by Petschek for the steady-state case to incorporate the effects of impulsive reconnection. We consider separately the convective zone and the diffusion region, and then match the results to obtain a self-consistent expression for the reconnection rate. The latter extends Petschek's result so that major assumptions, such as the establishment of a steady state, can be dropped and the model can be applied to the host of natural phenomena resulting from impulsive reconnection. Finally, major features resulting from time-varying reconnection are discussed, and the influence of cosmic rays on biological systems is considered.

\section{Exobiology of Titan}

\section{Simakov}

Group of Exobiology, Institute of Cytology Tikhoretsky Av.,

4 St.Petersburg,194064, Russia,E-mail:exobio@mail.ru

Titan, the largest satellite of Saturn, has a great exobiological significance. Its dense atmosphere has a large similarity to Earths primordial one. The main attention from an exobiological point of view is now devoted to a very complex atmospheric chemistry. A large number of minor constituents in the gas and solid phases such as carbon monoxide, carbon dioxide, ethane, ethylene, acetylene, cyanoacetylene, hydrogen cyanide, and many others were identified in the atmosphere. Accretion models of the Saturnian satellite suggest that heating released during late stages of its formation was sufficient to create a warm, dense atmosphere with a mass at least thirty times greater than the present value and a large open ocean on its surface. The first stages of chemical evolution would have taken place in this atmosphere and ocean under action of such energy sources as ultraviolet radiation, solar wind, galactic cosmic rays, magnetospheric plasma ion bombardment, electrical discharges and radiogenic heat. During the phase of cooling, Titan's ocean was roofed over with an icy crust. If life had originated by then, it could survive in some places up to the present. On Earth microbial life exists in all locations where microbes can survive. In other cases the variety of prebiotic processes can take place on Titan at the present time. The compositions of the rich atmosphere, which is host to extensive organic photochemistry and internal liquid layer, must be very complex and Titan's putative ocean might harbor life or complex prebiotic structures. The most recent models of the Titan's interior lead to the conclusion that a substantial liquid layer exists today under a relatively thin ice cover inside Titan. Mass balance calculations that model extraction of elements into the aqueous phase from chondritic material show that Titan's extensive subsurface ocean probably contains dissolved salts from endogenic materials incorporated into the satellite during its formation and released at the time of planetary differentiation. The low and hightemperature alteration of primitive accreted material leads to formation of a complex water solution of such cations as $\mathrm{K}, \mathrm{Na}, \mathrm{Mg}, \mathrm{Ca}, \mathrm{Mn}, \mathrm{Fe}$ and anions as $\mathrm{SO}_{4}^{2-}, \mathrm{Cl}^{-}, \mathrm{Br}^{-}, \mathrm{CO}_{3}^{2-}, \mathrm{HCO}^{3-}$ and others. Phosphorus, sulphur, micro- and macronutrients have to be abundant inside the base of Titan's rocks. There are three sources of organic carbon for Titan's ocean: complex atmospheric chemistry, carbonaceous chondrites and cometary bodies and seafloor hydrothermal systems. Since the light energy in the form of solar radiation is not accessible in such conditions the chemical energy has to be the main source, which drives the life and other disequilibria processes. So, the initial components for the origin of lithoautotrophic processes, such as $\mathrm{NO}_{3}^{-}, \mathrm{SO}_{4}^{2-}, \mathrm{CO}_{3}^{2-}$ could exist in the Titan's putative ocean from earlier stages of its evolution and provide biologically useful electron donor-acceptor pairs in the upper layer where the temperature and pressure are not very hostile. Nitrate accumulated in the ocean at the first stage of atmosphere's evolution would have allowed the first protobiosystems to use it as the source of energy. We would like to propose the idea that the first protoliving systems had internal energy source, namely, the chemical potential of an inorganic reaction. The basic reaction of nitrate reduction $\mathrm{NO}_{3}^{-} \rightarrow \mathrm{N}_{2}$ is more thermodynamically favourable in the row of different inorganic substrate. Electron acceptors such as $\mathrm{NO}_{3}^{-}, \mathrm{SO}_{4}^{2-}$, $\mathrm{Fe}_{3}^{+}, \mathrm{Mn}_{4}^{+}$, or $\mathrm{CO}_{2}$ have to be coupled with the electron donors. Electron donors that may be important in such a process include $\mathrm{H}_{2}$, $\mathrm{CO}, \mathrm{CH}_{4}, \mathrm{Fe}(\mathrm{II}), \mathrm{Mn}(\mathrm{II})$, pyrite, sulfur compounds and organic material. The possible biogeochemical cycle would demand only a few flow of external energy and reagent sources. The reaction of nitrate/ nitrite reduction could have served not only as an energy source but also as a source of reduced (or 'fixed') nitrogen compounds, which are essential for the synthesis of biologically important ones. Such putative life inside Titan does not depend on solar energy and photosynthesis for its primary energy supply and it is essentially independent of the surface circumstances. There could be microorganisms having a great similarity with the last common ancestor (LCA) on Earth. Along with the upper layer of the internal water ocean when temperature and pressure are suitable for living processes there are some additional appropriate sites for biological and/or prebiological activity at present: (1) water pockets and liquid veins inside an icy layer; (2) the places of cryogenic volcanism; (3) macro-, mini- and microcaves in an icy layer connecting with cryovolcanic processes; (4) the brine-filled cracks in an icy crust caused by tidal forces; (5) liquid water pools on the surface originating from meteoritic strikes; (6) the sites of hydrothermal activity on the bottom of the ocean. All environments mentioned above indicate that all conditions capable of supporting life are possible on Titan. All requirements needed for exobiology - liquid water which exists within a long geological period, complex organic and inorganic chemistry and energy sources for the support of biological processes are on Saturn's moon. On Earth life exists in all niches where water exists in liquid form for at least a portion of the year. Subglacial life may be widespread among such planetary bodies as Jovanian and Saturnian satellites and satellites of others giant planets, detected in our Galaxy in the last decade. The existence of a rich atmosphere is the main difference from Jupiter's moons. This atmosphere could supply the large quantity of different organic compounds to putative ocean. The existence of an ocean inside Titan may be indicated by Cassini fly-by data, but the detection of the signs of life on the surface or in such an ocean will demand new, more complicated missions.

\section{From Stars to Habitable Planet, the Austrian contribution to the COROT mission}

M. B. Steller(1), H. Ottacher(1), J. Heihsler(1), W. W. Weiss(2) (1) Department of Experimental Space Research, Space Research Institute Graz, Austrian Academy of Sciences, Schmiedlstr. 6, A-8042 Graz, Austria, E-mail: manfred.steller@oeaw.ac.at; (2) Institute for Astronomy, University of Vienna, Türkenschanzstr. 17, 1180 Vienna, Austria,E-mail:weiss@astro.univie.ac.at

When in 2005 the COROT spacecraft will be launched, this will be an important step towards the detection of telluric exoplanets. A highprecision, wide-field, stellar photometer, designed to perform continuous long-term observations of star systems will be onboard the spacecraft. Over a period of five months, the COROT instrument will observe and analyse the brightness variations of up to 12000 targets 
simultaneously. Every thirty-two seconds an image will be taken for the search of exoplanets, and the data will be processed in real-time onboard the spacecraft. Only statistical results will be transmitted to Earth. One element of the processing chain is the Boites Extracteur (BEX), a computer system for extracting the star images out of the CCD data stream, which is developed by the Space Research Institute in Graz. An in-house developed FPGA logic based pre-processor performs the separation of useful pixels from others, using pre-defined look-up tables. The pre-processor design supports the selection of every single pixel at the CCD that allows any kind of sophisticated pattern to be used as a mask. The selected pixels are sorted to data sets representing a target window. The succeeding instrument in the processing chain performs the statistical treatment of a data set. The key issue in the BEX design was the logic design of the pre-processors and the definition of the lookup table format to provide a maximum of flexibility by reasonable hardware effort. Since the generation of the look-up table is a critical issue for the mission operation, a specific software package supporting interactive and automatic positioning of masks was developed.

\section{Columbia river basalt carbonate formation - implications for the understanding of carbonates on mars and the search for life}

\section{Starke(1), D. S. Mc Kay(2), S. J. Wentworth(3),}

K. L. Thomas-Keprta(3)

(1) Department of Biology, Philipps University Marburg, Germany,

E-mail: starke@stud-mailer.uni-marburg.de; (2) NASA Johnson

Space Center, Houston, Texas, USA ; (3) Lockheed Martin, Houston, Texas, USA

Introduction. Basalts from the Columbia River (CRB) Plateau contain minor amounts of carbonates of unknown origins. These carbonates are known to form in aqueous, deep subsurface environments, for example in fractures and voids; where microbes can also grow. Many types of microbes (lithoautothropic microbes e.g., sulphur-reducers, dissimulatory iron-reducers, methanogens) have been identified in water and basalts from hundreds of meters below the surface of the Columbia River Plateau. ${ }^{1}$ The role these organisms play in the precipitation of the carbonates is uncertain. Consequently, we have examined the petrology and chemistry of the CRB carbonates to elucidate their modes of formation. This study is directly relevant to meteorites and future samples returned from Mars. Materials and Methods. We analysed small chips (a few mm in size) and a polished thin section of CRB. The samples had been collected near Spokane, Washington. We used JEOL 6340F and Philips 40XL field emission gun scanning electron microscopes (FE-SEM) in combination with light element energy-dispersive X-ray spectroscopy (EDS) systems. The thin section was also analysed with a petrographic microscope using transmitted, reflected and polarized light. The Cameca 100SX electron microprobe was used for wavelength-dispersive X-ray element mapping of the thin section and for quantitative mineral analyses. Results: Analysis of the CRB chips and the thin section showed that the carbonates are composed of siderite $\left(\mathrm{FeCO}_{3}\right)$, which are surrounded by a characteristic reddish rind. EDS and microprobe analysis of the rind indicates that the rind is Si-rich with minor Al. In the search for microbes in the carbonate-bearing CRB samples, we found some likely biogenic features similar to biofilm or mucilage. EDS analysis confirmed the presence of carbon, consistent with a biological origin. Discussion and conclusion. The relationship between the Si-rich rind and the carbonate indicates that some, if not all, of the rind was formed after the carbonate. The carbonate in the chip formed euhedral crystal faces, which means that it was able to grow in open space. The Si-rich rind was probably emplaced at fairly low temperatures, low enough to preserve siderite (decomposition temperature $\sim 500{ }^{\circ} \mathrm{C}$ ), because the carbonates show no detectable alteration at contacts. The layered Si-rich rind surrounding the carbonates could be a glass or a gel. We suggest that CRB carbonate globules probably formed by episodic precipitation from $\mathrm{Fe}$-rich fluids with intermittent precipitation of the $\mathrm{Si}-\mathrm{Fe}$-rich rims. However, the only likely biogenic features we found in our samples were traces of biofilm not obviously related to the carbonates. The textural, morphological, and chemical analyses are helping define the mode of
CRB carbonate formation, which can be applied to understanding carbonates on Mars.

References

${ }^{1}$ Stevens, T. O. and McKinley, J. P. (1995), Science 270, 450-454.

${ }^{2}$ V. Starke, D. S. McKay, S. J. Wentworth, K. L. Thomas-Keprta (2002) Carbonate Globules in Columbia River Basalt The Search for life in terrestrial subsurfaces environments as possible Martian Analogues. 33rd Annual Lunar and Planetary Science Conference, March 11-15, 2002, Houston, Texas, abstract no. 1475.

\section{Water on Europa}

\section{J. Sundstrand}

Department of Space Physics, Umeå University, Box 812, SE-981 28

Kiruna,Sweden,E-mail:00lis@ryp.umu.se

Discovered by Galileo and Marius in 1610, Europa became one of the six of Jupiter's known satellites. It has a composition, which is probably similar to the other terrestrial planets, but it also has a thin outer layer of ice. This makes its surface unlike anything in the inner solar system. The descriptions of the moon's surface strongly resemble the sea ice on Earth. It is possible that beneath the icy surface there is a layer of liquid water, perhaps as much as $50 \mathrm{~km}$ deep, kept liquid by tidally produced heat from Jupiter. If so, it would be the only place in the solar system, besides Earth, where liquid water exists in significant quantities. Europa has a very weak atmosphere composed of oxygen. Unlike the oxygen in Earth's atmosphere, Jupiter's moon is surely not of biologic origin. Most of it is generated by sunlight and charged particles hitting its icy surface producing a water steam, which splits into two parts, hydrogen and oxygen where the hydrogen escapes and leaves the oxygen. The exact nature of the moon's surface and interior is not yet obvious but the evidence is very strong for a subsurface ocean. One of the most powerful insights comes out from 15 years of research of volcanoes in the occurrence of liquid water, which can sustain life. Whether life can begin in such systems is controversial, but the evidence is clear about the extraction between volcanic processes and plentiful carbon-based life forms on and below the seafloor in the surrounding area of active spreading centres. This makes a long list of practical problems; how to land on Europa, go through the ice and explore the surface or the subsurface, what experiments should be attempted and how to manage the whole mission?

\section{Detection of past biological activity in Martian sediments under cathodoluminescence \\ R. Thomas, P. Gille, V. Barbin \\ Laboratoire de Physique et Chimie de l'Environnement (CNRS), LPCE 3A, Avenue de la Recherche Scientifique, 45071 Orléans cedex 2, France,E-mail:pgille@cnrs-orleans.fr}

The cathodoluminescence $(\mathrm{CL})$ consists in irradiating the sample with an electron beam of an energy range between 10 and $20 \mathrm{keV}$. This irradiating process induces a luminescence in the visible wavelength. The spectrum analysis of this luminescence gives data on the mineralogical composition; images at different scales (microscopic or macroscopic) give data on the growing process. CL has become an important standard technique for studying geological materials, offering a rather wide spectrum of applications. However, it is in the field of sedimentology and petrography that CL has proved to be especially valuable. These capabilities are very interesting for Martian exploration since, so far, no present results give evidence of the presence of carbonates and of biomarkers while these questions remain of a prime importance for Mars history. Looking for a fossil biological activity may be approached by the search of cells or fossil cells, as it was unsuccessfully done, but also by the study of the results of their activity and their interface with the mineralogical environment. Such very old bio-sedimentations as stromatolites or microbialites are known among the oldest terrestrial fossils and testify to the earliest terrestrial bioactivity. A discovery of such biosedimentations on the Martian surface would be of a prime interest for the exobiology concept. CL seems to be such a relevant method, allowing us to reach these analytic goals and fitting well with robotic facilities. It could be an optional subsystem to any optical microscopic experiment 
using an autonomous electron lamp. Preliminary results concerning the design of a CL instrument in compliance with the requirements of an in-situ Martian experiment and with the scientific goals are available.

\section{Photo ecological characterization of an epilithic ecosystem at a high mountain locality (central Spain)} R. de la Torre Noetzel(1), G. Horneck(2), L. G. Sancho(3), P. Rettberg(2), K. Scherer(2), T. Urlings(2), R. Facius(2)

(1) Instituto Nacional de Técnica Aeroespacial (INTA), Spanish Aerospace Research Establishment CRTA. AJALVIR, KM.

4 28850-Torrejón de Ardoz, Madrid, Spain, E-mail: torrenr@inta.es; (2) Institute for Aerospace Medicine, DLR, D-51170 Cologne, Germany; (3) Dpto. Biología Vegetal II, Universidad Complutense de Madrid, Madrid, Spain

Epilithic ecosystems in high mountains are exposed to extreme solar UV radiation and microclimatic conditions. Study of effects of UV radiation in parallel with microclimatic measurements is not common in experimental field conditions, especially in the Mediterranean region. In this study, we have investigated whether the cortex protects the protobiont against potential UV radiation damage. For this purpose, UV radiation was measured during a growing season at Sierra de Gredos $(2000 \mathrm{~m}$ a.s.l., $40.5 \mathrm{~N}, 5.5 \mathrm{~W}$ ), close to lichens samples of Rhizocarpon geographicum, with the DLR-Biofilm (Bacillus subtilis spores, type TKJ 6312 and type HA-101). This is a biodosimeter that quantifies the biologically effective UV dose (BED) (DNA target). An electronic UV dosimeter (Optometer, Giga-Herzt Optik, Puchheim, Germany), was used as an international standardized measure with a sensitivity similar to erythema, simultaneously for calibration. Microclimate (temperature, relative humidity) at the experimental side was determined by a microclimatic station (Squirrel, U.K.), including also a PAR sensor (Sky sensor). The application of Radiative Transfer Modelling for estimation of UV radiation climate for ideal conditions (i.e. clear sky), provide ratios of UV-B/UV-A irradiances about 100 times lower than the ratios of BED obtained with the TKJ 6312 Biofilm Ecodosimeter (DLR), demonstrating the high biological effectiveness of the UV-B range. Using lichen samples with and without cortex showed a substantial protection of the cortex against stress conditions (mainly microclimate), especially at summer solstice. Because of this high resistance of $\mathrm{R}$ geographicum against environmental UV-radiation, it might be an ideal model system for studies on the possibility of interplanetary transfer of life. Therefore, its sensitivity to the space environment will be tested during the upcoming BIOPAN mission of ESA.

\section{The discovery of water on the Galilean satellites by the Galileo magnetometer}

M. Volwerk(1), M. G. Kivelson(2,3), K. K. Khurana(2), C. Zimmer(4) (1) Department of Experimental Space Research, Space Research Institute, Austrian Academy of Sciences, Schmiedlstr. 6, A-8042 Graz, Austria,E-mail:martin.volwerk@assoc.oeaw.ac.at; (2) Institute for Geophysics and Planetary Physics, UCLA, Los Angeles, USA;

(3) Department of Earth and Space Sciences, UCLA, Los Angeles, USA,E-mail:mkivelson@igpp.ucla.edu,kkhurana@igpp.ucla.edu, (4) Institut Pasteur, Laboratoire Analyse d'Images Quantitative, Paris, France,E-mail: czimmer@pasteur.fr

During its tour around Jupiter, Galileo encountered the three icy satellites (Europa, Ganymede and Callisto) several times. The magnetometer data were used to study the interaction of the background Jovian field and the moons. Interestingly we found that the magnetic field in the neighbourhood of Europa and Callisto could well be described as the sum of the background magnetic field and an extra dipolar field created by the moon. This dipolar field changed direction when the background field changed direction, and this lead to the conclusion that we are dealing with an inductive magnetic field. This magnetic field is created by the inductive effect in a layer of liquid water, with some electrolytes, inside the moons. For Ganymede we found that the moon has its own internal magnetic dipole and the search for an inductive effect was more complicated. We found, by modelling the magnetic field that also for Ganymede we can describe the magnetic field as the sum of the background Jovian field, an internal dipole field and an extra dipolar field created by the inductive effect of a liquid water layer under the icy surface of this largest moon in the solar system.

\section{Is chiral prebiotic material produced by ion-molecule reactions in a magnetic field?}

\section{J.-E. Wahlund}

Swedish Institute of Space Physics (IRF) Uppsala Division, Box 537 SE-751 21, Uppsala, Sweden, E-mail: jwe@irfu.se

We bring forward a new hypothesis for the origin of homochirality of biomolecules which is a prerequisite for the origin of life on Earth and possibly elsewhere. We propose that the chiral pre-biotic molecules can be produced through ion-molecule reactions in a thin partial plasma medium in the pressence of a magnetic field by the action of the Lorentz force. Although the static magnetic field itself may only produce a very weak asymmetry in molecular reactions, it may cause a systematic and strong asymmetry if a second force (e.g., gravity, convection electric field, or centrifugal force for gyrating ions) exists. An ionized plasma state of the chemistry is therefore necessary in this scenario. This production of pre-biotic molecules, i.e. simple amino acids, may have occurred either in the proto-planetary nebula where comets formed or, more likely, in the ionosphere of the early Earth when life evolved about 3.9 Gyrs ago. Due to the low collision frequency in such an ionized medium, molecules and heavy ions would preserve their chirality for a relatively long time as compared to the lifetime at the surface level of Earth. A constant supply of chiral atmospheric products to the surface is therefore possible from the ionosphere. The low collision frequency also result in that of the background forces (e.g. electromagnetic forces) dominating the chemical reactions in the upper ionised part of the atmosphere (i.e. ionosphere), and this is what we expect on Titan and possibly early Earth. We also point out conditions for laboratory tests of this hypothesis and other observations in the solar system that could support our hypothesis.

\section{Comparative I6S rRNA analysis of extremophiles in the winter cover of a high mountain lake S. Waldhuber \\ University of Innsbruck, Institute of Zoology and Limnology, Technikerstr. 25, 6020 Innsbruck, Austria, E-mail: \\ sebastian.waldhuber@uibk.ac.at}

Until the late 1980s it was not even known that the winter cover (ice- and snow cover) of high mountain lakes is a temporary ecosystem settled with a whole variety of microorganisms. Lake Gossenkölle $(2413 \mathrm{~m})$ in the Austrian Alps is covered with ice up to two meters about 8 months a year. The structure of alternating ice- and slush layers favors the microbial community. In these slush layers liquid water around freezing point is available and isolated by the overlaying ice and snow cover. Especially in spring these slush layers become a highly productive ecosystem due to the accumulation of nutrients from the lake catchment area. The microbial activity from March/April until icebreak is even higher than in the water column below and comparable to mesotrophic lakes. In this study we present first results of the diversity of the microbial community in this temporary extreme habitat by comparative $16 \mathrm{~S}$ rRNA analysis.

\section{The Swedish Astrobiology Network (SWAN) 2 F. Wallinder \\ Department of Natural Sciences, Orebro University, 701 82, Orebro, Sweden, E-mail: fredrik.wallinder@nat.oru.se}

The Swedish Astrobiology Network was founded in 2001. The members are Swedish scientists with an interest in astrobiology, and come from disciplines such as astronomy, molecular biology, geology and theoretical philosophy. The members investigate subjects such as the deep biosphere, extremophiles, prebiotic chemistry and origins of life, planet and star formation in connection with astrochemistry. The network is supported by various organizations, but has no funding opportunities at present. The aim of the network is to promote astrobiology through interdisciplinary meetings and conferences, cooperative projects, 
interaction with sponsor agencies and spreading information to the general public. The network web address is www.astrobiologi.nu.

\section{Viability and DNA damage of halobacteria under physical stress conditions, including a simulated Martian atmosphere}

G. Weidler(1), S. Leuko(1), C. Radax(1), G. Kargl(2), N. Kömle(2), H. Stan-Lotter (1)

(1) Institute of Genetics and General Biology, University of Salzburg, Hellbrunnerstr.34, A-5020 Salzburg, Austria,E-mail:weidler@aon.at; (2) Department for Extraterrestrial Physics, Space Research Institute, Austrian Academy of Sciences, Schmiedlstr. 6, A-8042 Graz, Austria Several viable halobacteria were isolated from alpine rock salt of the Permo-Triassic age and described as novel species. ${ }^{1,2}$ They have apparently survived in the salt sediments over extremely long periods of time. Halobacteria could therefore be suitable model organisms for exploring the possibility of long-term survival of microorganisms on other planets. In addition, the discovery of extraterrestrial halite makes it plausible to consider a specific search for halophiles, perhaps in the planned sample return missions to Mars. We tried to develop experimental procedures to test the behaviour of halobacteria under Martian conditions. Cells of two species of haloarchaea were used: 1. Halobacterium sp. NRC-1, whose whole genome sequence is already known; ${ }^{3}$ 2. Halococcus dombrowskii, a novel isolate from Austrian PermoTriassic rock salt. ${ }^{2}$ Cells were grown in complex medium, containing $4 \mathrm{M}$ $\mathrm{NaCl}$, to an optical density of about 1 or 0.1 , respectively. For exposure experiments, cells were suspended in a buffer containing $50 \mathrm{mM}$ Tris$\mathrm{HCl}$, pH 8.0, $4 \mathrm{M} \mathrm{NaCl}$, without (TN) or with $80 \mathrm{mM} \mathrm{Mg}^{++}$(TNM). Aliquots of the cell suspensions were kept at minus $70{ }^{\circ} \mathrm{C}$ for up to seven days, or freeze-dried in a lyophilizer. In addition, exposure experiments of halobacterial cells in a liquid nitrogen cooled Space Simulation were begun at the Space Research Institute of the Austrian Academy of Sciences in Graz. The conditions obtainable in the Mars chamber are: temperature from about +5 to minus $100{ }^{\circ} \mathrm{C}$; pressure about $6-8 \mathrm{mbar}$, using a carbon dioxide atmosphere (or other gas mixtures). Survival of cells is evaluated by determination of colony-forming units, microscopic examination of cellular morphology; following staining with the LIVEDEAD kit (see also Poster by Leuko et al.), and examination of potential strand breaks in DNA using pulsed-field gel electrophoresis (PFGE). Results showed a reduction of viable cells, following deep freezing, or lyophilization respectively by a factor of only about 100 (depending somewhat on the presence of protective substances). Data will be presented on the DNA from stressed halobacterial cells, following digestion by restriction enzymes and separation by PFGE.

\section{References}

${ }^{1}$ Stan-Lotter, H., McGenity, T. J., Legat, A., Denner, E. B. M., Glaser, K., Stetter, K. O., Wanner, G. (1999) Very similar strains of Halococcus salifodinae are found in geographically separated Permo-Triassic salt deposits. Microbiology 145, 3565-3574.

${ }^{2}$ Stan-Lotter, H., Pfaffenhuemer, M., Legat, A., Busse, H. J., Radax, C., Gruber, C. (2002) Halococcus dombrowskii sp. nov., an Archaeal Isolate from a Permo-Triassic Alpine Salt deposit. Int. J. System. Evol. Microbiol. (in press).

${ }^{3}$ Ng, W. V., Kennedy, S. P., Mahairas, G. G., Berquist, B., Pan, M., Shukla, H. D., Lasky, S. R., Baliga, N. S., Thorsson, V., Sbrogna, J. et al. (2000) Genome sequence of Halobacterium species NRC-1. Proc. Natl. Acad. Sci., USA 97: 12176-81.

\section{MOST and COROT: involvement of the Vienna Institute for Astronomy in the search for extra-solar planets \\ W.W.Weiss(1), A. Scholz(2), M. Steller(3)}

(1) Institute for Astronomy, University of Vienna, Türkenschanzstr. 17, 1180 Vienna, Austria, E-mail: weiss@astro.univie.ac.at; (2) Institute of Communications and Radio-Frequency Engineering, Technical University Vienna; (3) Department of Experimental Space Research, Space Research Institute, Austrian Academy of Sciences, Schmiedlstr. 6, A-8042 Graz, Austria
MOST (Microvariability \& Oscillations of STars) is a Canadian project to perform high precision photometry from space. It was developed under J. Matthews, mission scientist at UBC, Vancouver. A $15-\mathrm{cm}$ Maksutov telescope is mounted in a microsatellite bus only $50 \mathrm{~kg}$ in mass and with dimensions of about $60 \times 60 \times 24 \mathrm{~cm}$. The microsat will be launched into a polar sun-synchronous orbit $\sim 800 \mathrm{~km}$ high, as a primary payload aboard Rocket launcher in March 2003. From that vantage point, it will be able to monitor certain stars in Continuous Viewing Zones for up to 8 weeks without interruption. Starlight will be directed onto a Fabry microlens (a few 100 microns across) to produce an image of the telescope pupil on a CCD, virtually eliminating photometric noise due to pixel-to-pixel sensitivity variations on the detector. Adjacent microlenses will produce comparable images of background sky fields, to correct for scattered Earthlight and zodiacal light. The Institute for Astronomy is involved in MOST by contributing a ground control station, presently developed at the Institute of Communications and Radio-Frequency Engineering, The importance of the Vienna site lies in the capability to increase the amount of observations significantly, because MOST will be visible in Vienna when neither of the Canadian ground stations in Toronto and Vancouver can communicate with the satellite. COROT (COnvection, ROtation and planetary Transits) has been proposed and studied in the framework of the French CNES minisatellite programme with participation of five European institutions and of Brasil. The consortium is lead by the PI, A. Baglin, Observatoire de Paris-Meudon. COROT has an entrance pupil of $27 \mathrm{~cm}$ and is a whitelight wide-field photometer, with a set of four frame-transfer CCDs as detectors. It will be launched in 2005 on a low-earth polar inertial orbit, allowing the monitoring continuously for about five months of stellar fields near the pole of the orbit. The Austrian hardware contribution to COROT is being developed at the Space Research Institute in Graz and is presented in more detail in another poster. Both space experiments, MOST and COROT, are designed for high-precision photometry which is needed to detect and/or investigate transits of planets. In the search for extra-solar planets, the detection of telluric exoplanets is the next significant challenge after the discovery of Jovian-sized exoplanets. COROT will be able to detect Earth-size planets close to their parent star, similar to Mercury, and planets slightly larger than the Earth in the Habitable Zone. MOST, on the other hand, is designed to investigate with hitherto unprecedented photometric accuracy known exoplanetary systems with the goal to discover further (smaller) planets or even moons through occultation. Simulations have shown that it will be possible to extract global information on the atmospheres of planets from such light curves. Both missions play an important role in preparing the ESA mission EDDINGTON, which is scheduled for launch in 2008.

\section{Identifying signs of fossil microbial life of terrestrial and extraterrestrial origin}

F. Westall, and the Archean Consortium

Centre de Biophysique Moléculaire, CNRS, Rue Charles Sadron, 45071 Orléans cedex02, France, E-mail: westall@cnrs-orleans.fr

The Archaean Consortium*: the Search for Life on the early Earth is represented by single-celled organisms morphologically similar to bacteria. Potential early life on two other terrestrial planets (Mars and Venus), as well as on Europa, would probably also have consisted of single-celled organisms. The controversy over interpretations of evidence for life in Martian meteorite ALH84001 has highlighted a problem, which was already recognized with respect to the identification of ancient terrestrial microfossils. A number of recent studies questioning (1) the evidence for an isotopic signature in 3.8 (or 3.65) Gyr. old rocks from SW Greenland, ${ }^{1}$ and (2) the petrographic evidence for fossil cyanobacteria in 3.46 Gyr. old cherts from the Pilbara in Australia ${ }^{2}$ have further underlined the difficulties involved. In dealing with early life, we recognise two related problems: (1) the nature and distinction of prebiotic signatures, and (2) the unambiguous identification of signatures of past life. Whereas it is highly unlikely that prebiotic signatures have been preserved on Earth (the rocks holding them were destroyed by later plate tectonic processes), such structures could well be preserved on Mars. We 
are presently working on the characterisation of biological signals in the oldest, well-preserved terrestrial sediments from the 3.5-3.3 Gy.-old Barberton and Pilbara greenstone belts using or will be using, the following methods: morphological investigation (high resolution SEM imaging), organic carbon investigations (mostly in situ: CFM, TEM), in-situ transition and heavy metal ( $\mathrm{S}, \mathrm{Fe}, \mathrm{Cu}, \mathrm{Zn}, \mathrm{Se}, \mathrm{Mo}, \mathrm{As}, \mathrm{U}, \mathrm{Tl}, \mathrm{Zn}$, $\mathrm{Ni}, \mathrm{Cu}, \mathrm{Ag}, \mathrm{Cd}, \mathrm{Pt}, \mathrm{Au}$ ) detection (PIXE), carbon, sulphur and nitrogen isotopes, trace elements, and Raman spectroscopy. This set of different approaches will hopefully elucidate the key criteria for the unambiguous identification of life. Future developments of some of these methods, as well as others, to analyse signs of fossil life on a nanoscale are also considered. Experimental studies will be undertaken to distinguish between prebiotic and biogenic signatures, in which prebiotic molecules will be fossilized under conditions imitating those present on the early terrestrial planets. The same array of techniques will be used to investigate the resulting microfossils. Thus, the use of multi-expertise studies on the same rock and experimental samples will provide a database of techniques and observations that will be of prime importance in the analysis of future sample return missions.

* The Archaean Consortium: 'The Search for Life on the early Earth', consists of: Exobiology Group Orléans, F. Westall, A. Brack, B. Barbier, M. Bertrand, A. Chabin, J.-N. Rouzaud,(all CNRS) J. Trichet, C. Défarges, J.-Robert Disnar, E. Vergès, J.-M. Beny (all ISTO), D. Pinti, B. Orberger, P. Gautret, Univ. Paris Sud, M.-C. Maurel, IJM Paris, N. Grassineau, RHC, London, J. Toporski, A. Steele, Carnegie, Washington and G. Southam, Univ. W. Ontario, Canada.

\section{The detection of organic materials in halite and epsomite test crystals}

A. D. Wilkins(1), A. Wright(1), J. Parnell(1), R. Artz(2)

(1) Department of Geology and Petroleum Geology, University of Aberdeen, King College, Aberdeen UK, E-mail:gmi377@abdn.ac.uk; (2) Department of Plant and Soil Science, Cruickshank Building, St. Machar Drive, Aberdeen, UK

Many potential strategies in the search for life on Mars involve the analysis in situ of samples on the Martian surface or the analysis of returned samples in a controlled environment. In particular, analyses seek evidence of organic carbon biomolecules and/or tangible life. Such organic material would be within the matrix of the Martian soil, embedded within solid rock as endoliths or fossils, or preserved within fluid inclusions in minerals. The range of techniques that could be employed in the analysis of organic matter is large, including Raman spectroscopy, Gas Chromatography-Mass Spectrometry (GC-MS), time-of-flight mass spectrometry, immunoassay, and molecular imprinted polymer sensors. Materials, which are surrogates of Martian mineralogy are currently being tested, especially meteorites and those materials found in extreme environments that may be analogues for Martian, surface conditions at some stage. In order to further prove the capabilities of analytical techniques to detect biomolecules or life, it is possible to grow test crystals in the laboratory, which incorporate organic materials that are a target for analysis. These crystals must have the advantage of being reproducible by a standard method, and hence can be made in large quantity, to allow progressive refinement of analytical techniques against a standard sample, and comparison of different techniques against the same sample. The crystals grown in this study are of compounds that form the evaporite minerals halite (sodium chloride) and epsomite (hydrated magnesium sulphate). Both of these evaporite minerals have a high solubility and can be readily precipitated, and as such they are ideal candidates for laboratory experiments. Halite and epsomite are of particular relevance to astrobiological testing, as they both occur in Martian meteorites, and are thought to be a significant component of Martian soils. The understanding that salt minerals are present within the Martian soils has been derived from a high abundance of sulphur and chlorine, and hence sulphates and chlorides, from the in-situ analysis of the Martian soils at various landing sites such as Viking 1, Viking 2 and Mars Pathfinder. Evaporites in general are proposed as an important target for astrobiological exploration. They grow in surface water-rich environments that could have supported life, and several studies of terrestrial halite suggest that it can preserve microbial life on a geological time scale. Their existence at the present Martian surface has been extensively discussed and they are an objective for at least one missionlanding site. Organic materials can be incorporated in crystal precipitates in several ways: (1) Encapsulation of living or recently dead microbial life as crystals grow. The microbes could be within solid crystal, or fluid inclusions within the crystals. (2) Incorporation of biomolecules, representing degradation or excretion products of life, in the ambient water that becomes entrapped in fluid inclusions. (3) As the host mineral is also a precipitate from the water, some biomolecules could also be within the solid crystal. (4) Micro-fracturing of crystals during burial may allow microbial colonization of fracture surfaces that are subsequently sealed in the crystal upon healing of the fractures. This study is concerned with organic materials that are incorporated during primary crystal growth, rather than subsequently as in the case of the healed fractures. Accordingly it has been sought to demonstrate the incorporation of microbial life and organic chemicals into fluid inclusions during crystal growth. The ability of organic compounds and bacteria to become entrapped, within fluid inclusions associated with primary crystal growth, in halite and epsomite has been determined experimentally: (1) Halophilic bacteria were observed to be trapped both within the solid crystal and fluid inclusions of halite. (2) Subsequent dissolution of the halite released bacteria whose presence was detected by staining for DNA. (3) The organic compound fluorescein was successfully entrapped within epsomite fluid inclusions. (4) The trapping of organic compounds implies the potential to detect them by GC-MS techniques. This success in detecting organic compounds and bacteria within the primary fluid inclusions of evaporite minerals has emphasised the viability of searching for life or past life within palaeolake basins on Mars.

\section{Biodevices for the detection of cosmic radiation effect utilizing} A. Zanini(1), D. Esposito(2), F. Fasolo(1), G. Torzillo(3), M. T. Giardi(2)

(1) INFN sez. di Torino, Via P. Giuria 1, 10125 Torino, Italy, E-mail: zanini@to.infn.it; (2) IBEV,CNR Montelibretti,via Salaria, km 29, 300 CP 10 00016, Monteronto, Roma, Italy; (3) ISE-CNR Scandicci, (FI), Via Salaria km 29, 3, 00016 Monterotondo Scalo, Italy In the present communication we report the development of (1) a biosensor/biodosemeter for the detection of ionizing radiation (biodosemeter) utilizing the advantageous properties of the Photosystem II (PSII) complex and its response to ionizing radiation, and (2) a biodevice to determine the effects of space stress on the oxygen-evolving activity of photosynthetic organisms (cyanobacteria and green algae). A biodevice is an apparatus that can reveal a biochemical variable using an intact biological component interfaced with an electronic transducer. It gives an electric signal that is easy to process, depending on the analysed variable. The biodevice is characterized by the sensibility and selectivity of the biological component response, together with easy use, versatility, miniature size and low cost. In this case, PSII particles and cells of photosynthetic oxygenic organisms compose the biological part. Fluorescence signal, which is correlated to photosynthetic activity, is used as a transducer system. The development of the biodosemeter is based on the following concepts: exposure of biological material to ionizing radiation leads to a loss of function due to the destruction of critical structures. Radiation target theory predicts an exponential decrease in biochemical activity that is dependent on the absorbed radiation energy and directly proportional to the mass of the individual molecules possessing this activity. The activity is lost whenever the protein is hit since a very high energy is transferred through the chain. Analysis of these changes makes it possible to establish a relationship between the dose of radiation received by the sample and the changes produced in a specific wavelength of the visible spectrum. On the other hand, the biodevice automatically measures the space radiation effect on living photosynthetic organisms, which are used as biomass and oxygen-producing system on shuttles. 
The biodevice(s), can give information on the photosynthetic organisms able to survive space stress, focalizing the choice of organisms useful for long-term missions. They are calibrated with several radiation beams (gamma of various doses, low and high energy neutrons, protons and heavy ions), to show separately the effect of radiation of different LET (Linear Energy Transfer) on the biochemical activity. Preliminary results of exposure to different beams are presented: (1) Am/Be source at Ispra Joint Research Centre (Varese, Italy): fast neutrons (energies between 1 and $4 \mathrm{MeV}$ ) (2) Co-60 source at Ispra Joint Research Centre (Varese, Italy): gamma rays (energies $1 \mathrm{MeV}$ ) (3) Linac accelerator at $\mathrm{S}$. Giovanni A. S. Hospital (Torino, Italy): gamma rays (energies under $18 \mathrm{MeV}$ ) (4) CERF - SPS beam at CERN (Geneva, Switzerland): fast neutrons (energies under $800 \mathrm{MeV}$ ) Protons and heavy ions of intermediate energy at LNL Laboratory, Legnaro, Italy.

Acknowledgements

ASI and ESA BIOPAN-FOTON M1 space mission supported the presented project.

\section{EPR study of gas-grain reactions explaining the synthesis of organic molecules in the interstellar medium \\ R. A. Zhitnikov \\ Ioffe Physico-Technical Institute, Russian Academy of Sciences, 26 Politekhnicheskaya, St. Petersburg 194021, Russia, E-mail: \\ zhitnikv.mares@pop.ioffe.rssi.ru}

For laboratory detection and investigation of free radicals, which often appear in chemical reactions of astrophysical interest either as important intermediate or as final products, we pioneered the use of the electron paramagnetic resonance (EPR) technique, which is very effective in observing these substances. ${ }^{1}$ This has allowed both formyl (HCO and DCO) and methyl $\left(\mathrm{CH}_{3}\right.$ and $\left.\mathrm{CD}_{3}\right)$ free radicals to be detected in a sequence of low-temperature gas-grain reactions of $\mathrm{H}$ and $\mathrm{D}$ atom addition in solid CO. The solid samples subjected to the EPR study were obtained by simultaneous independent deposition of $\mathrm{CO}$ molecules and either $\mathrm{H}$ or $\mathrm{D}$ atoms on the substrate cooled to liquid helium temperatures. The sequence of the $\mathrm{H}$-atom addition gas-grain reactions in solid $\mathrm{CO}$ is acknowledged to be among the fundamental processes responsible for the synthesis of organic molecules in interstellar cloud dust grains. The present observation of the above free radicals confirms that the solid-state gas-grain sequence of the reactions is efficient at low temperatures; it also suggests that there should be considerable concentrations of formyl and methyl radicals in the ISM. Another study concentrated on the formation of ethyl free radicals $\left(\mathrm{C}_{2} \mathrm{H}_{5}\right)$ in a low-temperature gas-grain reaction of an $\mathrm{H}$-atom abstraction from a $\mathrm{C}_{2} \mathrm{H}_{6}$ molecule by free $\mathrm{H}$-atom in solid $\mathrm{CH}_{4}$. These experiments were carried out by deposition onto a substrate, cooled by liquid helium, of a flow of $\mathrm{CH}_{4}$ molecules containing a small amount of impurities such as ethane molecules $\left(\mathrm{C}_{2} \mathrm{H}_{6}\right)$, free $\mathrm{H}$-atoms and $\mathrm{CH}_{3}$ radicals formed in a discharge in pure gaseous methane. EPR spectra of $\mathrm{CH}_{3}$ radicals, $\mathrm{H}$ atoms, and $\mathrm{C}_{2} \mathrm{H}_{5}$ radicals matrix-isolated in solid $\mathrm{CH}_{4}$ were detected. The relative concentrations of the radicals were found to depend on the experimental conditions. The abstraction reaction, $\mathrm{C}_{2} \mathrm{H}_{6}+\mathrm{H} \rightarrow$ $\mathrm{C}_{2} \mathrm{H}_{5}+\mathrm{H}_{2}$, took place in $\mathrm{CH}_{4}$-ice.

Reference

1 R. A. Zhitnikov and Yu. A. Dmitriev, Astronomy and Astrophysics 386, 1129 (2002).

\section{Possible environments of carbonaceous hondritis accumulation \\ S. Zhmur \\ Institute of the Lithosphere of Marginal Seas, Moscow, 119180,} Staromonetny,Russia,E-mail: zhmur@ilran.ru

The remnants of microbial organisms in carbonaceous hondrites (Mastigocladus, Phormidium, Lyngbya, Obrachuvella, Synehococcus, Gloecapsa-type) and the composition of the mineral matrix testify to the fact that ancient biosphere of extraterrestrial space objects existed in the form of cyanobaterial benthic communities (mats) and it was closely linked with hydrothermal environments. Cyanobacterial mats of ancient terrestrial hydrotherms played a significant part in the formation of ancient C-rich rocks of the Earth too. This gives us the ground to suppose that accumulation conditions of these terrestrial rocks could have taken place in carbonaceous hondrites formation as well. These environments could be: low-temperature terrestrial hydrothermal sources of active volcanic zones, where accumulation of Early Proterozoic shungites rocks was taking place with the help of coccoid cyanobacterial mat with Gloecapsa-type predominant; high-temperature underwater hydrotherms of lakes, similar to those, where Paleozoic graphite of Botogol massive of nefeline sienite and Early Archean graphite of Aldan shield were being formed. The source of organic matter in both cases was filamentous mat with predominant microorganisms of Phormidium-type; thermal geyser sources, where selfsealing biogenic-geyser silica structure are being formed; as is seen on the example of Early Proterozoic fine fibrous kerits of Volyn, which present mummified remnants filamentous mats composed of cyanobacteria Phormidium, Lyngbya, Mastigocladus, Obrachuvella-type, these structures promoted preservation of mats biomass. 